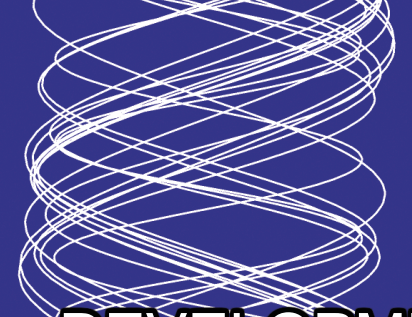

19UVEIEOMMENT OF A CENTRIFUGAL IN-LINE SEEATRATOR FOR OIL-WATER FLOWS

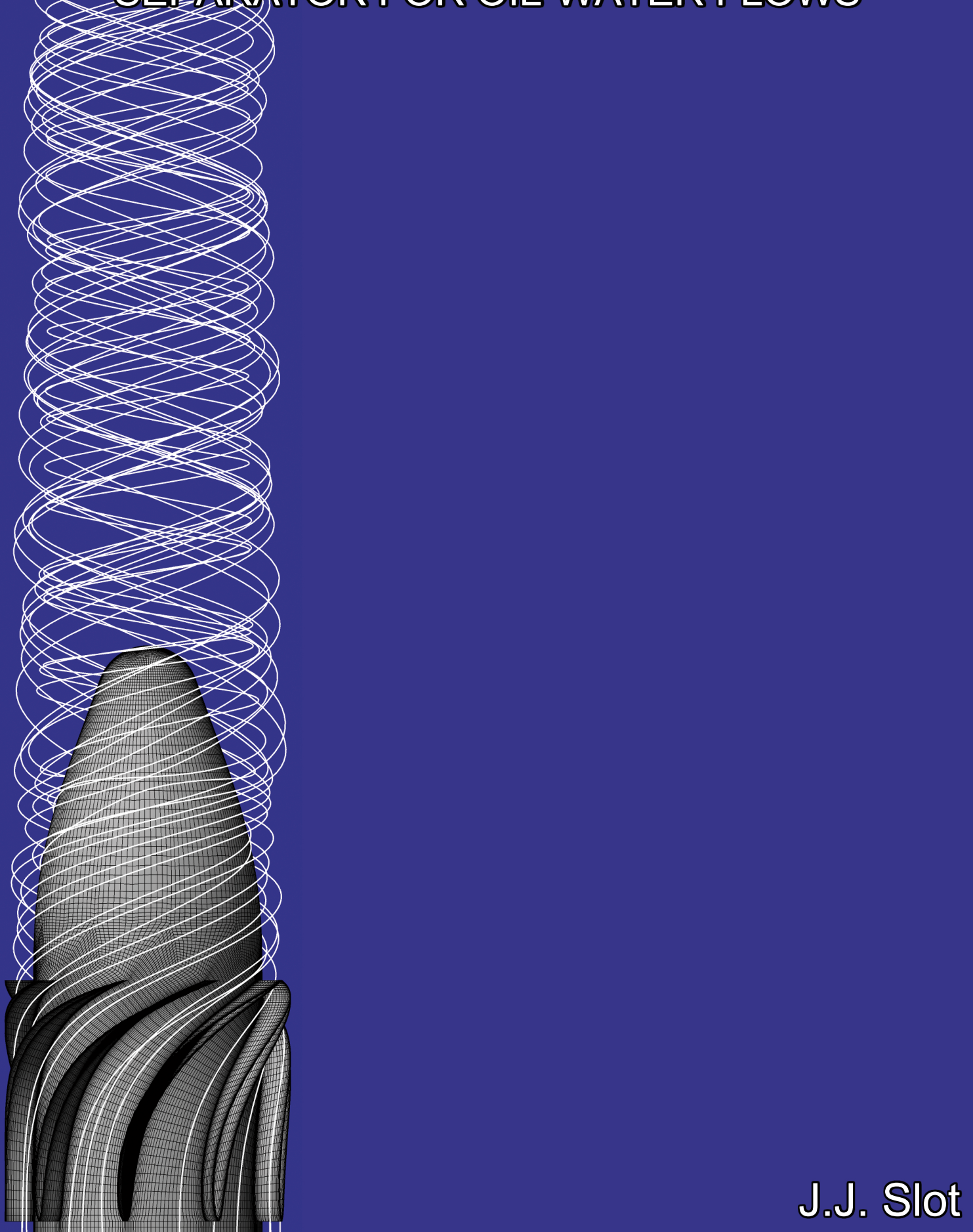




\section{DEVELOPMENT OF A CENTRIFUGAL IN-LINE SEPARATOR FOR OIL-WATER FLOWS}


DEVELOPMENT OF A CENTRIFUGAL IN-LINE SEPARATOR FOR OIL-WATER FLOWS J.J. Slot

Printed by Ipskamp Drukkers, Enschede

Thesis University of Twente, Enschede - With ref. - With summary in Dutch. ISBN 978-90-365-3542-7

copyright (C) 2013 by J.J.Slot 


\section{DEVELOPMENT OF A CENTRIFUGAL IN-LINE SEPARATOR FOR OIL-WATER FLOWS}

\section{PROEFSCHRIFT}

ter verkrijging van

de graad van doctor aan de Universiteit Twente,

op gezag van de rector magnificus,

prof. dr. H. Brinksma,

volgens besluit van het College voor Promoties

in het openbaar te verdedigen

op woensdag 22 mei 2013 om 16:45 uur

door

Jesse Jonathan Slot

geboren op 5 oktober 1981

te Enschede 
Dit proefschrift is goedgekeurd door de promotoren:

prof. dr. ir. H.W.M. Hoeijmakers

prof. dr. R.F. Mudde 


\section{Summary}

The world energy consumption will increase in the next decades. However, many aging oil fields are showing a steady decline in oil production. Furthermore, new, easily-accessible fields are discovered at a decreasing rate. Therefore, the oil and gas industry is developing new technology to extract the maximal amount of oil from an oil field in order to extend the economic lifespan of the wells.

Over time, many oil fields will produce increasing amounts of water, making the separation of the oil from the oil-water mixture an important processing step. In-line separation equipment using swirling flow can provide a light-weight, compact solution for offshore platforms and deepsea production sites, for which the use of large gravity-based separation vessels is very costly. In a collaborative ISPT (Institute of Sustainable Process Technology) project between the University of Twente (UT), Delft University of Technology (TUD), Wageningen University and Research Centre (WUR) and parties from the oil and gas industry such an in-line separator has been investigated numerically and experimentally. In this thesis the work carried out at the UT on the numerical part using computational fluid dynamics, is discussed. This work has been done in close cooperation with Laurens van Campen, who has carried out measurements in a large-scale flow loop at the TUD and with Thomas Krebs, who has investigated experimentally oil-water emulsions at the WUR.

The numerical simulations employ the time-dependent Reynolds-averaged Navier-Stokes equations for both single and two-phase flow. The anisotropic SSG Reynolds stress turbulence model is used to describe the effect of turbulence on the mean flow field quantities. The separator consists of a pipe with an internal diameter of $100 \mathrm{~mm}$, in which the internal swirl element (ISE) is placed. The ISE is equipped with vanes, which generate the swirling flow. Downstream of the ISE, due to the centrifugal force the oil flows towards the center of the $1.7 \mathrm{~m}$ long pipe. At the end of this pipe, the oil is extracted by a concentrically placed pick-up tube.

Based on the incoming oil droplet size, fluid properties, length of the separator, pipe diameter and other parameters, the required centrifugal force, and therefore the flow deflection produced by the ISE, are computed using an engineering approach. A potential flow method is used to design the shape of the vanes, which cross-section is based on an airfoil of the NACA four-digit airfoil series. Care is taken in the design of the nose and tail section of the ISE to smoothly guide the flow past the ISE and to avoid the introduction of disturbances in the flow.

The performance of the in-line separator is compared to that of a hydrocyclone design in term of separation efficiency and pressure drop. It turned out that the more compact ISE has a lower total pressure drop and shows separation efficiency comparable to that of the hydrocyclone.

The results of numerical simulations of single-phase flow show reasonable agreement with LDA measurements of the flow in the experimental rig at the TUD. The radial distribution of the axial velocity is a $\mathrm{W}$-shaped distribution with an annular region of reversed flow and positive axial velocity in the center of the pipe and near the wall. The distribution of the azimuthal velocity shows a solid-body rotation in the center and a potential-flow 
vortex type of distribution at larger radius.

A study varying the flow rate has demonstrated that, when scaled with the inlet velocity, the velocity distributions for high Reynolds numbers almost collapse onto a single curve. The flow split FS is defined as the ratio between the mass flux extracted through the pick-up tube and the mass flux at the inlet. For increasing FS, the axial and azimuthal velocity components in the center increase considerably, while the flow away from the center shows only small changes. As a result of the increase in azimuthal velocity, the centrifugal force in the center increases substantially, almost tripling in magnitude when FS in increased from 0.2 to 0.4 .

The velocity distribution downstream of the ISE depends heavily on the ratio of the axial and azimuthal velocity at the trailing edge of the vanes. This ratio is defined as the swirl angle Sw. Decreasing Sw from the design value of 6.72, results in a decrease in both the maximum positive and negative values in the axial velocity distribution, that is, the distribution becomes flatter. The flow reversal disappears when $\mathrm{Sw}$ is decreased further. For very low Sw, the radial distribution of the axial velocity is V-shaped instead of W-shaped. The parameter studies have shown that the flow pattern in the separator is complex and that the dependance of the flow pattern on Re, FS and $\mathrm{Sw}$ is complicated.

For two-phase flow, the modeling of the interfacial force terms has been investigated. Compared to the results of emulsion compression experiments at the WUR, the use of the Ishii-Zuber drag law in the numerical simulations leads to an overprediction of the oil volume fraction. Also, a maximum oil volume fraction as function of the centrifugal force is observed in the experiments. However, this is not seen in the numerical results. The numerical results obtained using the Ishii-Zuber drag law therefore require critical review. Furthermore, the use of an isotropic turbulent dispersion model results in excessive diffusion of the oil volume fraction. The development of a model for anisotropic turbulent dispersion is recommended for future work. Finally, in Ansys CFX 14.0 the use of the virtual mass force leads to an unphysical distribution of the oil volume fraction near the pipe wall. Its use is therefore not recommended until this issue is resolved.

In the in-line separator, the radial distribution of the velocity for two-phase flow is similar to that of single-phase flow. As expected, the separation efficiency increases for increasing FS. However, the numerical results overpredict the separation efficiency, in some extreme cases by $50 \%$. For a high oil volume fraction at the inlet, the numerical results show a flooding phenomenon, which gives a decrease in separation efficiency. This feature has not been observed in the experiments.

In the experiments droplet break-up has been observed, which causes a mismatch between the droplet size in the experimental work and the one assumed in the numerical work. A reduction in the size of the mono-dispersed droplets in the numerical simulations to values representative for ones in the flow loop at the TUD, decreases the differences in results between experimental and numerical work substantially. Although, the separation efficiency is still overpredicted by the numerical simulations compared to the experiments. Furthermore, the drag law fails to reproduce the behavior of the emulsion seen in the center of the pipe in the experiments. Further research is needed to improve the modeling of the drag between the phases in order to accurately predict the oil volume fraction. The inclusion of an anisotropic turbulent dispersion model in the computation may improve 
the numerical predictions.

The evolution of the droplet size distribution can be described by the use of population balance models. In the present work a method of classes has been used. Only turbulenceinduced coalescence is currently accounted for in the modeling. The coalescence model of Coulaloglou and Tavlarides has been implemented in Ansys CFX in order to more accurately describe the coalescence behavior for liquid-liquid two-phase flows. Unfortunately, the break-up model did not function properly. Therefore, a simulation has been carried out with a droplet size of $50 \mu \mathrm{m}$ at the inlet, for which it is expected that break-up will not occur in the flow as it passes the ISE. Further downstream the turbulent dissipation rate is much lower and break-up, even of large droplets, is not expected to occur.

The result for poly-dispersed two-phase flow show a modest increase in the Sauter mean diameter from 50 to about $80 \mu \mathrm{m}$ in the center of the pipe. Further away from the axis of the separator the droplet growth is even smaller. The separation efficiency is, although improved, still quite close to the one for the two-phase flow simulation with mono-dispersed $50 \mu \mathrm{m}$ droplets.

The current modeling of the coalescence process in the densely packed emulsion in the center of the pipe is apparently incomplete. Further research is required to achieve a better prediction of the poly-dispersed flow in the center. Droplet break-up should be incorporated into future research. Although much progress has been made, the large differences between experimental and numerical results, limited understanding of dense emulsions in swirling, turbulent flow and long computational times raise questions about the feasibility of using transient CFD calculations to optimize the design of oil-water separators in an industrial environment. 


\section{Samenvatting}

De wereldwijde vraag naar energie zal de komende decennia toe blijven nemen. Vele oudere olievelden laten echter een dalende olie productie zien. Tevens worden er steeds minder nieuwe, gemakkelijk toegankelijke olievelden ontdekt. De olie en gas industrie ontwikkelt daarom nieuwe technologie om de economische levensduur van een veld te maximaliseren. $\mathrm{Na}$ verloop van tijd zal voor de meeste olievelden de hoeveelheid geproduceerd water toenemen. Dit maakt de scheiding van de olie uit het olie-water mengsel een belangrijke stap in de verwerking van de ruwe olie. In-line centrifugale scheidingsinstallaties kunnen compacte, lichtgewicht alternatieven bieden, die in te zetten zijn op plaatsen waar gangbare scheidingsvaten hoge kosten met zich mee brengen, bijvoorbeeld op boorplatforms en in sub-sea installaties. In een samenwerkingsproject van de Universiteit Twente (UT), de Technische Universiteit Delft (TUD), de Wageningen Universiteit en Research centrum (WUR) en de industrie is een dergelijke compacte, centrifugale olie-water scheider zowel numeriek als experimenteel onderzocht. Het onderzoek met betrekking tot het numerieke gedeelte, uitgevoerd aan de Universiteit Twente, wordt in dit proefschrift beschreven. Laurens van Campen heeft in Delft metingen verricht aan een experimentele opstelling van de scheider. Thomas Krebs heeft in Wageningen het gedrag onderzocht van oliewater emulsies.

De numerieke simulaties maken gebruik van de tijdsafhankelijke Reynolds-gemiddelde Navier-Stokes vergelijkingen voor zowel een- als twee-fase stromingen. Het anisotrope SSG Reynolds spanningsmodel is gebruikt om het effect van de turbulentie op de gemiddelde snelheden te representeren.

De olie-water scheider bestaat uit een pijp met een diameter van $100 \mathrm{~mm}$, waarbinnen een wervelgenerator is geplaatst. De interne wervelgenerator bestaat uit een centraal lichaam, met negen gekromde bladen tussen het centrale lichaam en de binnenwand van de pijp. De wervelgenerator produceert een roterende stroming in de pijp. In deze wervelstroming beweegt de olie naar het midden van de $1.7 \mathrm{~m}$ lange pijp, waar de olie wordt afgevangen door een centraal geplaatste extractiebuis. Een praktische aanpak is gevolgd om de benodigde centrifugale krachten, en daarmee de gewenste azimuthale snelheid van het olie-water mengsel te bepalen. Hierbij is rekening gehouden met de diameter van de binnenkomende druppels, de vloeistof eigenschappen, de scheider lengte en de pijp diameter. Een potentiaalstromingsmethode is gebruikt om de vorm van de bladen te ontwerpen. Deze bladen zijn gebaseerd op de NACA 4-serie. De neus en staart secties van de interne wervelgenerator zijn dusdanig ontworpen dat de stroming glad verloopt en dat verstoringen van de stroming worden vermeden. De prestaties van de centrifugaal scheider zijn vergeleken met die van een hydrocycloon in termen van scheidingsefficiëntie en drukval. Voor vergelijkbare scheidingsefficiëntie is de drukval over de compactere centrifugaal scheider kleiner.

Vergelijking van de resultaten van de numerieke simulaties en de resultaten van de experimenten laat voor een-fase stromingen een redelijke overeenkomst zien. De radiale verdeling van de axiale snelheid heeft een $W$-vorm met positieve snelheden in zowel het midden van de pijp als bij de wand en een annulair gebied met terugstroming. De radiale distributie van de azimuthale snelheid lijkt sterk op die van een potentiaal wervel met 
een vaste-lichaam rotatie in het centrum van de pijp. Een studie naar het effect van de variatie van het debiet laat zien dat, mits geschaald met de inlaat snelheid, de snelheidsverdelingen bij hoge Reynolds getallen op dezelfde kromme samenvallen.

De extractie ratio is gedefinieerd als de verhouding tussen de massa-flux welke de scheider verlaat door de extractie buis en de massa-flux door de inlaat. Bij toenemende extractie ratio nemen de axiale en azimuthale snelheden in het midden van de pijp meer dan evenredig toe, terwijl de snelheidsveranderingen in de buurt van de wand gering zijn. Ten gevolge van de toegenomen azimuthale snelheid neemt de centrifugale kracht drastisch toe. Voor een toename van de extractie ratio van 0.2 tot 0.4 , stijgt de maximale centrifugale kracht met een factor 3 .

Het stromingspatroon hangt sterk af van de verhouding tussen azimuthale en axiale snelheid van de stroming uit de wervelgenerator. Deze verhouding is gedefinieerd als de wervelhoek. Verlaging ten opzichte van de ontwerpwaarde van 6.72, leidt tot een afname van zowel de maximale positieve als negatieve snelheden. Het snelheidsprofiel in het dwarsvlak wordt dus vlakker. Het terugstroomgebied verdwijnt bij een verdere afname van de wervelhoek. De axiale snelheidsverdeling heeft zelfs een V-vorm in plaats van een $\mathrm{W}$-vorm voor zeer lage wervelhoek. Parameter studies hebben aangetoond dat het stromingspatroon in de scheider complex is en dat er geen simpele relaties zijn voor de afhankelijkheid van de stroming met betrekking tot Reynolds getal, extractie ratio en wervel hoek.

$\mathrm{Er}$ is een analyse uitgevoerd van de modelering van de interactie-krachten in twee-fase stromingen. In vergelijking met de resultaten van emulsie compressie experimenten uitgevoerd aan de WUR, leidt het gebruik van de correlatie voor de weerstand van Ishii en Zuber in de numerieke simulaties tot een te hoge olie volume fractie. Ook wordt er in de experimenten een maximale olie volume fractie gevonden, welke afhankelijk is van de grootte van de centrifugaal kracht. Dit effect is niet waargenomen in de numerieke simulaties. De resultaten verkregen met de Ishii-zuber correlatie voor de weerstand moeten kritisch worden geïnterpreteerd.

Als er in de numerieke simulaties gebruik wordt gemaakt van een isotroop turbulent dispersie model treedt er niet-realistische diffusie op van de olie volume fractie. De ontwikkeling van een anisotroop turbulent dispersie model wordt aanbevolen voor toekomstig onderzoek.

In de resultaten van numerieke simulaties met Ansys CFX 14.0, ontstaat er een nietfysische verdeling van de olie volume fractie bij de wand van de pijp als de toegevoegde massa van de gedispergeerde fase wordt meegenomen. Het advies is daarom om dit model niet te gebruiken totdat deze problemen zijn opgelost.

De snelheidsverdeling bij twee-fase stromingen zijn vergelijkbaar met die van de een-fase stroming. Zoals verwacht stijgt de scheidingsefficiëntie met toenemende extractie ratio. Echter, de scheidingsefficiëntie wordt numeriek te hoog voorspeld. De overschatting kan in het meeste extreme geval oplopen tot $50 \%$. Voor een hoge inlaat olie volume fractie laten de berekeningen een duidelijke daling zien in scheidingsefficiëntie, dit effect wordt niet gevonden in de metingen bij de TUD.

In de experimentele opstelling breken de olie druppels op in kleinere druppels, waardoor er een verschil ontstaat tussen de druppelgrootte in de experimenten en die in de berekenin- 
gen wordt gebruikt. Het gebruik van een druppelgrootte, welke meer overeenkomt met de druppelgrootte waargenomen in de experimentele opstelling, leidt tot een significante verkleining van het verschil in scheidingsefficiëntie tussen metingen en berekeningen. Maar de scheidingsefficiëntie wordt nog steeds overschat in de numerieke resultaten. De gebruikte modelering van de weerstandskrachten kan het gedrag van de emulsie in het midden van de pijp niet goed beschrijven. Daarom is verder onderzoek nodig om een betere modelering van de weerstandskrachten te krijgen en zodoende beter de olie volume fractie in het midden van de pijp te kunnen voorspellen. Ook kan het meenemen van anisotrope turbulente dispersie de nauwkeurigheid van de resultaten verbeteren.

De verandering van de druppelgrootte verdeling kan worden beschreven door gebruik te maken van populatie balansmodellen. In dit onderzoek is gebruik gemaakt van een zogenaamde klassen methode. Enkel coalescentie ten gevolge van turbulentie is meegenomen in de modelering. Het coalescentie model van Coulaloglou en Tavlarides is geïmplementeerd in Ansys CFX, omdat dit model een betere beschrijving van coalescentie in olie-water mengsels geeft. Helaas werkte in CFX het standaard model voor het opbreken van druppels niet goed. Daarom is een numerieke simulatie uitgevoerd met een poly-disperse verdeling van druppelgroottes, startend met een uniforme verdeling van druppels met een diameter van $50 \mu \mathrm{m}$ bij de inlaat. Er wordt verwacht dat voor deze druppelgrootte het opbreken van druppels niet optreedt als het olie-water mengsel door de interne wervelgenerator stroomt. Verder stroomafwaarts zullen ook grotere druppels stabiel zijn, omdat de turbulente dissipatie hier veel lager is.

De resultaten van de numerieke simulatie met een poly-disperse verdeling laten een beperkte groei in Sauter gemiddelde diameter zien, namelijk van 50 tot ongeveer 80 $\mu \mathrm{m}$ in de midden van de pijp. De druppelgroei neemt af naar de wand toe en is nihil bij de pijpwand. De scheidingsefficiëntie is weliswaar hoger, maar ligt dicht bij de scheidingsefficiëntie berekend voor een twee-fase stroming met een constante druppelgrootte van $50 \mu \mathrm{m}$.

De huidige beschrijving van het coalescentie proces in de dichtgepakte emulsie in het midden van de pijp is incompleet. Verder onderzoek is nodig om betere voorspellingen te kunnen doen van dergelijke twee-fase stromingen. Daarnaast moet in volgend onderzoek het opbreken van de druppels worden meegenomen. De grote verschillen tussen numerieke en experimentele resultaten, onvolledige kennis van coalescentie gedrag in dichte emulsies in roterende, turbulente stromingen en de zeer lange rekentijden maken dat verder onderzoek nodig is voordat de toepasbaarheid van tijdsafhankelijke numerieke stromingsberekeningen voor het optimaliseren van olie-water scheiders haalbaar is in een industriële omgeving. 
Samenvatting vi vin

Table of Contents vi vi vis

List of acronyms $\quad x$

Nomenclature $\quad$ xi

1 Introduction $\quad \mathbf{1}$

1.1 Oil production . . . . . . . . . . . . . . . . . . . . . . 1

1.1 .1 Geology ... . . . . . . . . . . . . . . 2

1.1.2 Gravity-based oil-water separation . . . . . . . . . . . . . . 2

1.2 Centrifugal in-line separation . . . . . . . . . . . . . . . . . 3

1.3 Project structure and objectives . . . . . . . . . . . . . . . . . . 4

1.4 Outline of thesis . . . . . . . . . . . . . . . . . . 5

2 Computational fluid dynamics $\quad 7$

2.1 Introduction . . . . . . . . . . . . . . . . . . . . . . . . . . 7

2.2 Single-phase Reynolds Averaged Navier-Stokes equations . . . . . . . . . 8

2.3 Turbulence modeling . . . . . . . . . . . . . . . . . . 9

2.3.1 Reynolds stress transport equation . . . . . . . . . . . . . . . . . 9

2.3 .2 Wall functions . . . . . . . . . . . . . . . . . . . . 13

2.4 Two-fluid model . . . . . . . . . . . . . . . . . . . . . . . . . . . . . . . . . . . . . 17

2.4.1 Volume averaging procedure . . . . . . . . . . . . . . . . 17

2.4 Interfacial coupling . . . . . . . . . . . . . . . 23 
2.4 .3 Multiphase turbulence modeling . . . . . . . . . . . . . . 28

2.5 Ansys CFX . . . . . . . . . . . . . . . . . . . . . . . . . . . . . . . . . 28

3 Design of separator 31

3.1 Introduction . . . . . . . . . . . . . . . . . . . . . . . . . . 31

3.2 Design of internal swirl element . . . . . . . . . . . . . . . . . . 32

3.2.1 Requirements of the velocity field . . . . . . . . . . . . . . . 32

3.2 .2 Design of vane geometry . . . . . . . . . . . . . . . . . . . . . 35

3.2.3 Design of nose section . . . . . . . . . . . . . . . . . . . 39

3.2.4 Design of tail section . . . . . . . . . . . . . . . . . . . . . . 40

3.3 Comparison with alternative separator geometries . . . . . . . . . . . . . 42

3.3.1 Alternative configuration . . . . . . . . . . . . . . . . . . . . . . . 42

3.3.2 Comparison results ... . . . . . . . . . . . . . . . . . 43

3.3.3 Conclusion . . . . . . . . . . . . . . . . . . . . 47

3.4 Experimental setup . . . . . . . . . . . . . . . . . . . . . . . . 48

3.4.1 Measurement techniques . . . . . . . . . . . . . . 49

4 Results for single-phase flow 51

4.1 Introduction . . . . . . . . . . . . . . . . . . . . . . 51

4.2 Preprocessing . . . . . . . . . . . . . . . . . . . . . . . . 51

4.2.1 Computational mesh . . . . . . . . . . . . . . . . . 52

4.2 .2 Solver settings . . . . . . . . . . . . . . . . . . . 54

4.2.3 Modeling of the flow through straightener . . . . . . . . . . . 55

4.3 Flow patterns ... . . . . . . . . . . . . . . . 56

4.3.1 Flow reversal . . . . . . . . . . . . . . . . . . . . . 57

4.3.2 Validation by comparison with experimental data . . . . . . . 59

4.3.3 Evaluation design approach . . . . . . . . . . . . . . . . 60

4.4 Velocity distributions . . . . . . . . . . . . . . . . . . . . . . 62

4.5 Pressure distribution . . . . . . . . . . . . . . . . . . . . . . 67

4.6 Flow near pick-up tube . . . . . . . . . . . . . . . . . . . . 71

4.7 Parameter studies . . . . . . . . . . . . . . . . . . . . . . . . . . . . . . . . . . 73

4.7.1 Dimension analysis . . . . . . . . . . . . . . . . . 73

4.7 .2 Variation of flow rate . . . . . . . . . . . . . . . . 74

4.7.3 Variation of flow split . . . . . . . . . . . . . . . . . . . . 79

4.7.4 Variation of swirl angle . . . . . . . . . . . . . . . . . . 84

4.8 Conclusions . . . . . . . . . . . . . . . . . . . . . . . . . . . . . . . . . . . . . 91

5 Results for two-phase flow 93

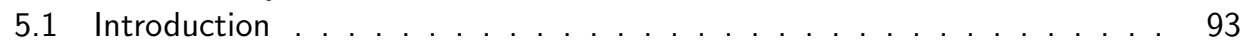

5.2 Evaluation of the modeling . . . . . . . . . . . . . . . 95

5.2 .1 Evaluation of drag law . . . . . . . . . . . . . . 95

5.2 .2 Turbulent dispersion . . . . . . . . . . . . . . . . . . . . . 102

5.2 .3 Virtual mass force . . . . . . . . . . . . . . . . . . . . 107

5.2 .4 Lift force . . . . . . . . . . . . . . . . . . . . . . 108

5.2 .5 Conclusions . . . . . . . . . . . . . . . . . . 109 
5.3 Results for two-phase flow . . . . . . . . . . . . . . . . . . . . 110

5.3.1 Separation performance . . . . . . . . . . . . . . . 111

5.3.2 Comparison of single and two-phase flow . . . . . . . . . . . . 113

5.4 Separation efficiency studies . . . . . . . . . . . . . . . . . . . . . . . . . . 119

5.4.1 Variation of the flow split . . . . . . . . . . . . . . . . . . . 119

5.4.2 Variation of the inlet oil volume fraction . . . . . . . . . . . . . 123

5.4.3 Validation by comparison with experimental data . . . . . . . . 126

5.4.4 Variation of the inlet droplet size . . . . . . . . . . . . . . . . . 132

5.4 .5 Conclusions . . . . . . . . . . . . . . . . . . . . . . 134

6 Coalescence and break-up 137

6.1 Introduction . . . . . . . . . . . . . . . . . . . . . . 137

6.2 Population balances . . . . . . . . . . . . . . . . . . . . . . . 138

6.2.1 Number density equations . . . . . . . . . . . . . . . . . . . . 138

6.2.2 Turbulence-induced coalescence . . . . . . . . . . . . . . . . . . 141

6.2 .3 Droplet break-up . . . . . . . . . . . . . . . . . . . . 146

6.3 Poly-dispersed two-phase flow . . . . . . . . . . . . . . . . . . . . . . . . 151

6.3.1 Poly-dispersed flow results . . . . . . . . . . . . . . . . . . 151

6.3.2 Limitations of population balance method . . . . . . . . . . . . 156

6.4 Conclusion . . . . . . . . . . . . . . . . . . . . . 157

$\begin{array}{lll}7 & \text { Concluding remarks and recommendations } & 159\end{array}$

$\begin{array}{ll}\text { Bibliography } & 163\end{array}$

A Design of nose section with cubic Hermite polynomials 173

B Design of tail section with quintic Hermite polynomials 175

$\begin{array}{ll}\text { C Sensitivity of velocity distribution for swirl angle Sw variation } & 177\end{array}$

$\begin{array}{ll}\text { Acknowledgments } & 179\end{array}$

$\begin{array}{ll}\text { About the author } & 180\end{array}$ 
CFD computational fluid dynamics

DQMOM direct quadrature method of moments

DSD droplet size distribution

FS flow split

HC-BVF hydrocyclone with blocked vortex finder

HC-OVF hydrocyclone with open vortex finder

HPO heavy phase outlet

ISE internal swirl element

ISPT Institute for Sustainable Process Technology

LDA laser doppler anemometry

LES large eddy simulations

LPO light phase outlet

MOM method of moments

NACA National Advisory Committee for Aeronautics

PBE population balance equations

PDMS polydimethylsiloxane

PMMA polymethylmethacrylate

PVC polyvinylchloride

QMOM quadrature method of moments

RANS Reynolds averaged Navier-Stokes

RSM Reynolds stress model

SDS sodium $n$-dodecyl sulfate

SSG Reynolds stress model by Speziale, Sarkar and Gatski [105]

TUD Delft University of Technology

UT University of Twente

VOF volume of fluid

WUR Wageningen University and Research Centre 


\section{Roman symbols}

$A_{d} \quad$ effective droplet cross-sectional area $\quad\left[\mathrm{m}^{2}\right]$

$A_{k, i} \quad$ Interfacial area of phase $k$ in averaging volume $\mathrm{V} \quad\left[\mathrm{m}^{2}\right]$

Ar Archimedes number, eq. (5.4)

a acceleration

$a_{i j} \quad$ normalized anisotropy tensor

$a_{r} \quad$ radial acceleration

$a_{\zeta} \quad$ constants used in thickness distribution $y_{t}$, for $\zeta=[1, \ldots, 4]$

$B \quad$ constant used in log-law; $B=5.2$

$B_{B} \quad$ source term for birth by droplet break-up in PBE

$B_{B}^{\prime} \quad$ size group source term for birth by droplet break-up in PBE

$B_{C} \quad$ source term for birth by droplet coalescence in PBE

$B_{C}^{\prime} \quad$ size group source term for birth by droplet coalescence in PBE

Bo Bond number, eq. (5.7)

$b_{\zeta} \quad$ constants in definition of nose shape, for $\zeta=[1,2]$

$C_{1} \quad$ adjustable parameter in two-fluid turbulent dispersion model

$C_{3} \quad$ empirical constant coalescence model [23]; $C_{3}=2.17 \times 10^{-4}$

$C_{4} \quad$ empirical constant coalescence model [23]; $C_{4}=2.28 \times 10^{13}$

$C_{D} \quad$ drag coefficient

$C_{\text {Dav }} \quad$ Constant in correlation for maximum droplet size by Davies [26]

$C_{\text {decay }}$ swirl decay parameter; $C_{\text {decay }}=0.04$

$C_{l} \quad$ lift coefficient

$\mathbf{C}_{\text {loss }}$ sum of individual loss coefficients $c_{\text {loss }}$

$C_{s} \quad$ constant used in $T_{i j k}^{\prime} ; C_{s}=0.22$

$C_{s \zeta} \quad$ constants used in $R_{i j}^{(s)}$ by Speziale et al. [105], for $\zeta=[1,2]$ 
$C_{r \zeta} \quad$ constants used in $R_{i j}^{(r)}$ by Speziale et al. [105], for $\zeta=[1, \ldots, 5] \quad[-]$

$C_{R} \quad$ constant used in $R_{i j}^{(s)}$ by Rotta [89]; $C_{R}=0.18 \quad$ [-]

$C_{R S} \quad$ constant used in turbulent dissipation transport equation; $C_{R S}=0.066 \quad[-]$

$C_{W e} \quad$ constant use by Davies [26]; $C_{W e}=1 \quad[-]$

$C_{\epsilon \zeta}$ constants used in turbulent dissipation transport equation, for $\zeta=[1,2] \quad[-]$

$C_{\mu} \quad$ constant used in $k-\epsilon$ turbulence model; $C_{\mu}=0.09$ [-]

$c$ chord line of vanes [m]

$\mathbf{c}_{\text {loss }}$ loss coefficient of contraction or expansion in pipe [-]

$D \quad$ pipe diameter [m]

$D_{B} \quad$ source term for death by droplet break-up in PBE $\quad\left[\mathrm{m}^{-3} \mathrm{~s}^{-1}\right]$

$D_{B}^{\prime} \quad$ size group source term for death by droplet break-up in PBE $\quad\left[\mathrm{m}^{-3} \mathrm{~s}^{-1}\right]$

$D_{C} \quad$ source term for death by droplet coalescence in PBE $\quad\left[\mathrm{m}^{-3} \mathrm{~s}^{-1}\right]$

$D_{C}^{\prime} \quad$ size group source term for death by droplet coalescence in PBE $\quad\left[\mathrm{m}^{-3} \mathrm{~s}^{-1}\right]$

$D_{S t} \quad$ Stokes' law

$D_{t} \quad$ turbulent mass diffusivity

$d \quad$ droplet diameter

$d_{32} \quad$ Sauter mean diameter

$d_{\max } \quad$ maximum droplet diameter

Eu Euler number

$F \quad$ compressive force in droplet collision

$F_{c e n} \quad$ centrifugal volume force acting on droplet

FS flow split

$f \quad$ friction factor

$f_{B V} \quad$ breakage mass fraction

$f_{l} \quad$ correction function of Mei [77] in lift force

$f_{m c} \quad$ rotation frequency of micro-centrifuge

$f_{n} \quad$ weight factor of Hermite polynomials for nose shape

$f_{p} \quad$ size group fraction of size group $p$

$\bar{f}_{p} \quad$ cumulative size group fraction of size groups $p_{\min }$ to $p$

$f_{t} \quad$ weight factor of Hermite polynomials for tail shape

$g \quad$ gravitational constant

$g(a: b) \quad$ specific break-up rate of droplets of mass $a$ into to $b$ and $b-a$

$h \quad$ reference height in hydrostatic pressure

$h_{0} \quad$ initial thickness of drainage film

$h_{c} \quad$ height of the compressed droplet layer in micro-centrifuge

$h_{f} \quad$ thickness of drainage film at rupture

$h_{t} \quad$ total height of the liquid column in micro-centrifuge

$g$ gravitational acceleration vector

$\mathbf{K}_{\text {loss }}$ tensor of loss coefficients in momentum loss term $S_{m}$

$k \quad$ turbulent kinetic energy

$L \quad$ pipe length

$\mathrm{L}^{\prime} \quad$ length ratio $\mathrm{L} / \mathrm{D}$

$l_{e} \quad$ characteristic eddy length scale [m]

$l_{s t r} \quad$ length of flow straightener 
$\mathcal{L} \quad$ length scale in compression force

$M_{k, i} \quad$ generalized interfacial drag term

$\left[\mathrm{N} \mathrm{m}^{-3}\right.$

$M_{\max }$ mass of largest size group

$[\mathrm{kg}]$

$M_{\text {min }}$ mass of smallest size group

$[\mathrm{kg}]$

$M_{p} \quad$ mass of size group $p$

$[\mathrm{kg}]$

$m \quad$ mass

$\dot{m} \quad$ mass flow rate

$m_{d} \quad$ mass of the oil droplet

$m_{c} \quad$ mass of of the displaced water by oil droplet

$[\mathrm{kg}]$

$[\mathrm{kg}]$

$m_{\max }$ mass of the largest oil droplet

$[\mathrm{kg}]$

$m_{\min } \quad$ mass of the smallest oil droplet

$[\mathrm{kg}]$

$m_{p} \quad$ mass of the droplet of size $p$

$N \quad$ number density size group

$[\mathrm{kg}]$

$n \quad$ number density of oil droplets

$\left[\mathrm{m}^{-3}\right]$

$n_{p} \quad$ number density of oil droplets with mass $m_{p}$

$n_{d} \quad$ number of droplets in droplet tracking analysis

$\left[\mathrm{m}^{-3}\right]$

$\mathbf{n}_{\mathbf{k}, \mathbf{i}} \quad$ outward normal of phase $k$ at the interface

$n_{\lambda} \quad$ number density of turbulent eddies

$P \quad$ production of turbulent kinetic energy

$P_{\zeta} \quad$ cubic Hermite polynomials, for $\zeta=[1,4]$

$P(a: b)$ breakage probability of droplet with mass $a$ breaking into $b$ and $a-b$

$P_{i j} \quad$ production tensor

$p \quad$ pressure

$p_{\text {tot }}$ total pressure

$p_{0} \quad$ reference pressure

$p^{\prime} \quad$ fluctuation pressure

$\langle p\rangle \quad$ time or area-averaged pressure

$\left\langle p_{\text {tot }}\right\rangle$ time or area-averaged total pressure

$\langle\bar{p}\rangle \quad$ area-averaged $\langle p\rangle$ over cross-flow plane

$\left\langle\bar{p}_{\text {tot }}\right\rangle$ area-averaged $\left\langle p_{\text {tot }}\right\rangle$ over cross-flow plane

$Q \quad$ flow rate

$\left[\mathrm{N} \mathrm{m}^{-2}\right]$ $\left[\mathrm{m}^{3} \mathrm{~s}^{-1}\right]$

$Q(a: b)$ specific coalescence rate of droplets of mass $a$ and $b$

$\left[\mathrm{m}^{3} \mathrm{~s}^{-1}\right]$

$Q_{\zeta} \quad$ quintic Hermite polynomials, for $\zeta=[1,6]$

$R \quad$ pipe radius

$R_{C} \quad$ radius of core of solid-body rotation

$R_{i j} \quad$ pressure-strain tensor

$R_{i n} \quad$ radius of central body in ISE

$R_{i j}^{(s)} \quad$ slow pressure or return-to-isotropy term

$r \quad$ radial coordinate

$r_{p q} \quad$ equivalent radius, given by equation (6.22)

$r_{m c} \quad$ radial coordinate in micro-centrifuge

$\mathrm{Re}_{\mathrm{d}} \quad$ Reynolds number based on droplet diameter

$\mathrm{Re}_{\mathrm{D}} \quad$ Reynolds number based on pipe diameter

Ro Rossby number 
S swirl number, defined in eq. (3.28)

$S_{i j} \quad$ mean strain tensor

$S_{m} \quad$ momentum loss term in porous region modeling

Sw swirl angle

$s \quad$ coordinate along chord line

$s_{i} \quad$ s-coordinate of lower or upper $(i=[l, u])$ surface of cascade vane

$s_{i}^{\prime} \quad$ rotated s-coordinate of lower or upper $(i=[l, u])$ surface of cascade vane $[\mathrm{m}]$

$T_{i j k} \quad$ stress flux tensor

$T_{i j k}^{\prime} \quad$ approximation of $T_{i j k}^{(p)}$ and $T_{i j k}^{(u)}$ in modeling

$\left[\mathrm{m}^{2} \mathrm{~s}^{-3}\right]$

$T_{i j k}^{(p)} \quad$ pressure transport tensor

$\left[\mathrm{m}^{2} \mathrm{~s}^{-3}\right]$

$\left[\mathrm{m}^{2} \mathrm{~s}^{-3}\right]$

$T_{i j k}^{(u)} \quad$ turbulent convection tensor

$\left[\mathrm{m}^{2} \mathrm{~s}^{-3}\right]$

$T_{i j k}^{(v)} \quad$ viscous diffusion tensor

$t$ time

$t_{p q} \quad$ time scale of coalescence

$\mathcal{U}$ velocity scale in compression force

$U_{\theta} \quad$ azimuthal velocity scale in design process

$\boldsymbol{u}$ flow velocity vector

$\boldsymbol{u}^{\prime} \quad$ fluctuation velocity vector

$\tilde{\boldsymbol{u}}^{\prime} \quad$ velocity increments in turbulent dispersion model

$\langle\boldsymbol{u}\rangle \quad$ time or area-averaged velocity vector

$\langle\tilde{\boldsymbol{u}}\rangle \quad$ instantaneous velocity in turbulent dispersion model

$\left\langle u_{1}\right\rangle \quad$ velocity tangential to the surface

$\left\langle u_{2}\right\rangle \quad$ velocity normal to the surface

$u_{i n} \quad$ velocity of the oil inside the droplet

$u_{z, L P O}$ prescribed axial velocity component at LPO

$u_{\infty} \quad$ terminal velocity of single droplet

$u_{\tau} \quad$ friction velocity

$u_{\lambda}^{\prime} \quad$ turbulent eddy velocity

$u_{\tau}^{*} \quad$ nominal friction velocity

$\left\langle\boldsymbol{u}^{\prime} \boldsymbol{u}^{\prime}\right\rangle$ Reynolds stresses

$V \quad$ arbitrary averaging volume

$\left[\mathrm{m}^{2} \mathrm{~s}^{-3}\right]$

$v \quad$ droplet volume

We Weber number

We $_{\text {cr }} \quad$ critical Weber number; $W_{\text {cr }}=1.2$

$X_{p q r} \quad$ fraction of the mass $M_{q}+M_{r}$ added to size group $p$ after coalescence

$x \quad \mathrm{x}$-coordinate in global frame

$x_{i n} \quad \mathrm{x}$-coordinate of vane contour on central body

$x_{\text {nose }} \quad \mathrm{x}$-coordinate of start of nose section

$x_{\text {tail }} \quad \mathrm{x}$-coordinate of start of tail section

$x_{\text {vane }} \mathrm{x}$-coordinate of start of vane section

$Y \quad$ camber line shape

$Y_{i} \quad$ y-coordinate of lower or upper $(i=[l, u])$ surface of cascade vane 
$\begin{array}{lll}Y_{i}^{\prime} & \text { rotated y-coordinate of lower or upper }(i=[l, u]) \text { surface of cascade vane } & {[\mathrm{m}]} \\ y & \text { y-coordinate in global frame } & {[\mathrm{m}]} \\ y_{i n} & \text { y-coordinate of vane contour on central body } & {[\mathrm{m}]} \\ y^{+} & \text {non-dimensional wall distance } & {[-]} \\ z & \text { axial coordinate in global frame } & {[\mathrm{m}]} \\ z_{i n} & \text { z-coordinate of vane contour on central body } & {[\mathrm{m}]}\end{array}$

\section{Greek symbols}

$\alpha \quad$ volume fraction

$\alpha_{0} \quad$ initial volume fraction of emulsion in micro-centrifuge

$\alpha_{c} \quad$ average volume fraction of emulsion in micro-centrifuge

$\alpha_{c, \max }$ maximum of average volume fraction of emulsion in micro-centrifuge

$\alpha_{p} \quad$ volume fraction of droplet size group $p$

$\alpha_{\text {inlet }}$ volume fraction at inlet

$\beta \quad$ number of variables in physical problem

$\boldsymbol{\Gamma} \quad$ normally-distributed random vector

$\gamma \quad$ porosity of porous region

$\gamma_{k} \quad$ phase indicator function of phase $k$

$\Delta m \quad$ maximum distance from the chord line $c$, normalized by $c$

$\Delta p \quad$ area-averaged pressure difference between inlet and LPO

$\Delta Q_{o} \quad$ oil imbalance

$\Delta t \quad$ time step

$\Delta u \quad$ velocity difference

$\Delta x_{n} \quad$ length of nose section ISE

$\Delta x_{t} \quad$ length of tail section ISE

$\delta \quad$ vane thickness distribution

$\delta_{\max }$ maximum thickness vane profile, normalized by $c$

$\epsilon \quad$ turbulent dissipation rate

$\epsilon_{i j} \quad$ turbulent dissipation tensor

$\epsilon_{S W} \quad$ angular momentum loss factor

$\eta \quad$ Kolmogorov length scale $\eta=\left(\nu^{3} / \epsilon\right)^{1 / 4}$

$\eta_{1} \quad$ separation efficiency measure, defined by eq. (3.31)

$\eta_{2} \quad$ separation efficiency measure, defined by eq. (3.33)

$\theta_{p q} \quad$ collision frequency between size groups $p$ and $q$

$\lambda \quad$ size of turbulent eddy

$\lambda_{\min } \quad$ smallest turbulent eddy in break up process

$\lambda_{p q} \quad$ coalescence efficiency between size groups $p$ and $q$ according to [23]

$\lambda_{p q}^{P B} \quad$ coalescence efficiency between size groups $p$ and $q$ according to [84]

$\kappa \quad$ constant used in log-law; $\kappa=0.41$

$\mu \quad$ dynamic viscosity

$\mu_{m} \quad$ mixture viscosity

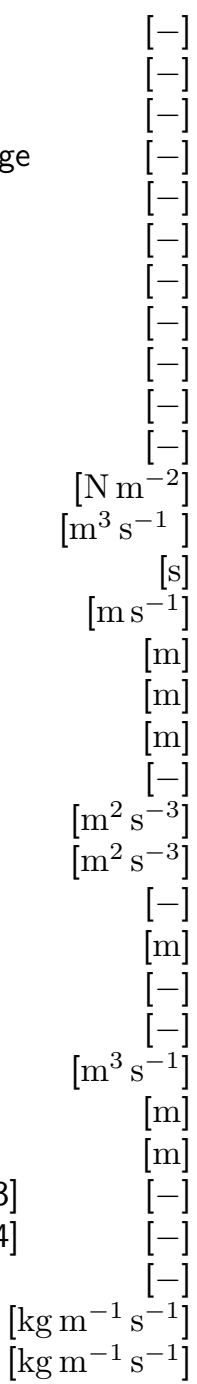




$\begin{array}{llr}\nu & \text { kinematic viscosity } & {\left[\mathrm{m}^{2} \mathrm{~s}^{-1}\right]} \\ \nu_{t} & \text { turbulent viscosity } & {\left[\mathrm{m}^{2} \mathrm{~s}^{-1}\right]} \\ \xi & \text { local coordinate system of averaging volume } V & {[\mathrm{~m}]} \\ \xi_{n} & \text { local coordinate of nose section of ISE } & {[-]} \\ \xi_{t} & \text { local coordinate of tail section of ISE } & {[-]} \\ \Pi & \text { number of non-dimensional variables } & {[-]} \\ \Pi_{i j} & \text { velocity-pressure gradient tensor } & {\left[\mathrm{m}^{2} \mathrm{~s}^{-3}\right]} \\ \rho & \text { density } & {\left[\mathrm{kg} \mathrm{m}^{-3}\right]} \\ \rho_{m} & \text { mixture density } & {\left[\mathrm{kg} \mathrm{m}^{-3}\right]} \\ \sigma & \text { interfacial tension } & {\left[\mathrm{N} \mathrm{m}^{-1}\right]} \\ \sigma_{t} & \text { turbulent Schmidt number } & {[-]} \\ \sigma_{\epsilon} & \text { constant in } k \text { - } \epsilon \text { turbulence model } & {[-]} \\ \tau_{d} & \text { droplet response time } & {[\mathrm{s}]} \\ \tau_{D a v} & \text { deformation-resisting stress as defined by } & \text { Davies }[26] \\ \tau_{e} & \text { eddy lifetime } & {\left[\mathrm{N} \mathrm{m}^{-2}\right]} \\ \tau_{i f} & \text { interfacial pressure acting on droplet } & {[\mathrm{s}]} \\ \tau_{i n} & \text { viscous stress inside oil droplet } & {\left[\mathrm{N} \mathrm{m}^{-2}\right]} \\ \tau_{i n t} & \text { droplet-eddy interaction time } & {\left[\mathrm{N} \mathrm{m}^{-2}\right]} \\ \tau_{p q} & \text { timescale of the collision } & {[\mathrm{s}]} \\ \tau_{p r e s} & \text { normal stresses acting on droplet } & {[\mathrm{s}]} \\ \tau_{t r} & \text { time for droplet to transverse eddy } & {\left[\mathrm{N} \mathrm{m}^{-2}\right]} \\ \tau_{w} & \text { wall shear stress } & {[\mathrm{s}]} \\ \tau_{\mu} & \text { tangential stresses acting on droplet } & {\left[\mathrm{N} \mathrm{m}^{-2}\right]} \\ \phi_{t} & \text { tip angle of the tail shape with respect to pipe axis } & {\left[\mathrm{N} \mathrm{m}^{-2}\right]} \\ \chi & \text { ratio of interfacial energy required for break-up to kinetic energy of eddy } & {\left[\mathrm{rad}^{2}\right]} \\ \chi_{c} & \text { critical } \chi & {[-]} \\ \psi & \text { gradient of camber line shape } & {[-]} \\ \omega & \text { vorticity } & {[-]} \\ \Omega_{i j} & \text { mean vorticity tensor } & {\left[\mathrm{s}^{-1}\right]} \\ \dot{\omega}_{B, \lambda} & \text { droplet-eddy collision frequency } & {\left[\mathrm{s}^{-1}\right]} \\ & & \end{array}$

\section{Subscripts}

$0 \quad$ initial condition

I daughter droplet formed after break-up

II daughter droplet formed after break-up

$A_{k, i} \quad$ surface-averaged over $A_{k, i}$

$B \quad$ break-up

$b$ bulk

C coalescence

$c \quad$ continuous

d droplet

in central body 


$\begin{array}{ll}k & \text { phase } k \\ l & \text { lower } \\ k, i & \text { interface of phase } k \\ m c & \text { micro-centrifuge } \\ n & \text { nose } \\ o & \text { oil } \\ r & \text { radial } \\ t & \text { tail } \\ t e & \text { trailing edge } \\ u & \text { upper } \\ w & \text { water } \\ z & \text { axial } \\ \theta & \text { azimuthal }\end{array}$

\section{Superscripts}

$h \quad$ harmonic term in modeling of $R_{i j}$

$n \quad$ normal to the wall

$P B \quad$ Prince and Blanch [84]

$r \quad$ rapid term in modeling of $R_{i j}$

$S^{\prime} \quad$ fluctuating part of quantity on $A_{k, i}$

$s \quad$ slow term in modeling of $R_{i j}$

$t$ tangential to the wall

$v l \quad$ vane-less 

CHAPTER 1

Introduction

\subsection{Oil production}

Crude oil is the world's primary energy source and is an essential raw material for many products such as plastics and pharmaceuticals. In 2007 the world use of liquid fuels was 86.1 million barrels per day. Furthermore, the worldwide demand for oil, while fluctuating due to economic circumstances, show an increasing trend. From 2007 to 2035, the world energy consumption is predicted to rise by $49 \%$ [22]. In addition to the increasing demand for oil, the number of easily accessible fields is decreasing. For example, in 2010 the proven world oil reserves are estimated to have increased by 12 billion barrels or about $1 \%$ since

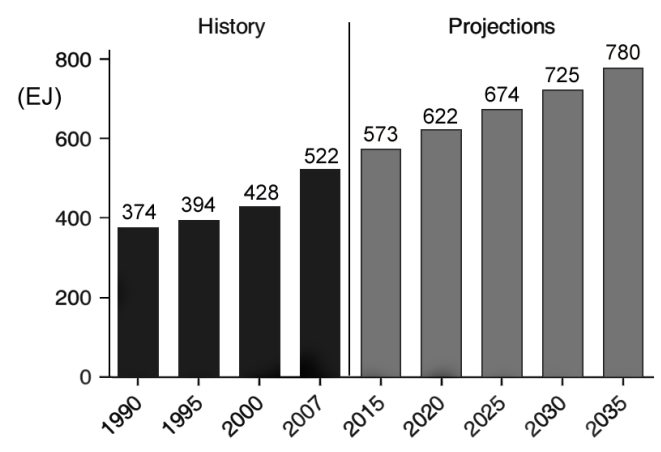

Figure 1.1: World marketed energy consumption in exajoule (EJ), 1990-2035. Adapted from [22]. 
2009 [22]. Therefore, the amount of oil extracted from each oilfield is being maximized to extend its economic lifespan.

\subsubsection{Geology}

In the Middle East oil was already used for lighting and for the waterproofing of boats five thousand year ago. In that region the oil was seeping out of the ground. However, in general the crude oil, along with gases and water, has to be extracted from porous reservoir rocks buried deep below the surface. These rocks are permeable so fluid can migrate into the reservoir. An essential feature of these reservoir rocks is the impermeable ceiling, trapping its content in the reservoir. These conditions can for instance be encountered in an anticline, as sketched in figure 1.2.

Due to the density differences, gas and crude oil will migrate to the upper levels of the reservoir, pushing down the water. Therefore, as oil fields mature, crude oil is produced with increasing quantities of water. This volume fraction of water can increase to over $80 \%[90,95]$. In the first stage of recovery a well is drilled through the impermeable layer. Initially, the well pressure is often high enough to push the crude oil to the surface. At a later stage, the well pressure decreases and other methods need to be employed to maintain an economic extraction rate. These methods include using beam pumps or the introduction in the reservoir of surface-tension reducing chemicals.

\subsubsection{Gravity-based oil-water separation}

Due to the high water cut, that is the volume fraction of water, the efficient separation of oil from water becomes an increasingly important processing step. The water is often salty, which may lead to corrosion of downstream equipment. Furthermore, the separation of the phases is required in order to reduce the demands on the transport facilities. The volume to be transported over long distances is greatly reduced by discharging cleaned, separated water into the environment. Alternatively, the separated water can be re-injected into the deeper layers of the reservoir in order to maintain the well pressure. In both cases the

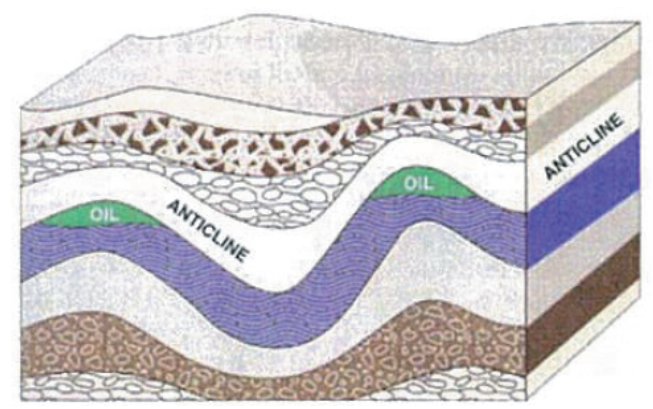

Figure 1.2: An anticline formed by an impermeable layer trapping hydrocarbons. After [102] 


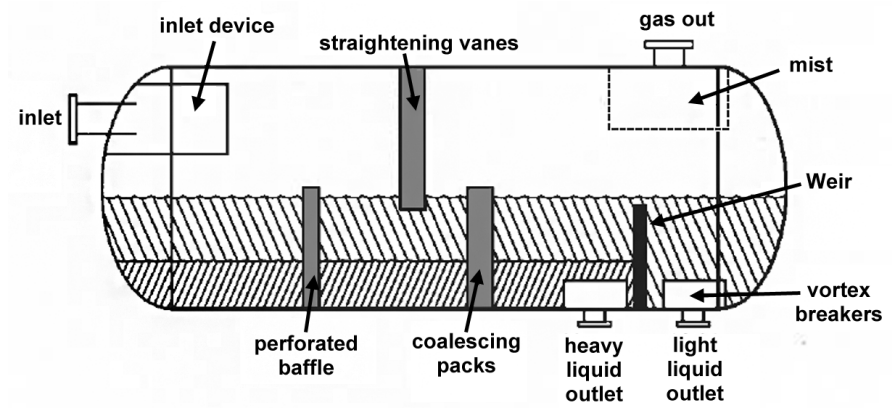

Figure 1.3: Sketch of a three-phase, gravity-based separation vessel. Adapted from [15].

water quality has to meet certain specifications.

The separation of the liquid phases presents some difficulties. The density difference between the oil and the water, which is the driving force behind mechanical separation, is only small. Furthermore, emulsification can be encountered. Sometimes the formation of these emulsions is exacerbated by chemicals added to the reservoir. The emulsions must be broken but poor coalescence properties can hinder this process. In addition, the separation equipment must be capable to deal with slugging, which introduces strong variations in volume fractions of the incoming feed.

Mostly, separation is achieved in very large vessels employing the action of gravity. This method has not changed significantly over the last decades. A sketch of such a vessel with its various internal components is given in figure 1.3. A mixture of oil, water and gas enters through an inlet device which distributes the incoming mixture evenly over the cross-section of the separator. A weir is placed to collect the water and to prevent spill over of water into the oil outlet. Often coalescing packs are installed to increase the capacity of the separator. These packs consist of inclined, perforated plates which drastically reduce the settling distance of the droplets. The droplets coalesce on these plates to form larger droplets or a continuous layer of liquid which can be separated more easily.

To attain a large separation capacity the separation vessels need to be very large as well. The corresponding large weight and space requirements of these vessels lead to high costs for off-shore processing facilities. The reasons are that the platform needs to be expanded to accommodate these facilities, while also the structure of the entire platform needs to be strengthened to carry the larger weight. This will in turn further increase the weight and therefore the costs.

\subsection{Centrifugal in-line separation}

The separation can also be achieved by the use of swirling flow. In that case the driving force behind separation of the phases is the centrifugal force which can easily be a hundred 
times larger than gravity. The lighter fluid will be forced to migrate to the center of the centrifugal force field, while the heavier fluid flows towards the outside. Subsequently, the phases can be extracted by separate outlet devices. Preferably, this method of separation is carried out in-line. For in-line separation piping is used instead of large vessels.

The higher body forces experienced by the dispersed phase leads to a strong reduction in residence time, and therefore to a reduction in footprint and weight, of the separation device while retaining the required capacity. The size reduction also leads to significant cost reduction. Additionally, the small size results in a lower amount of hydrocarbons present in the separator during operation. This reduction in volume diminishes the requirement for flare and vent facilities needed in case of a blow-down, hence leading to improved safety features. The smaller size of the equipment requires a lower level of control and monitoring instrumentation, which reduces the maintenance burden. Also by utilizing piping to achieve separation, the need for costly inspections of the large separation vessels is eliminated.

One of the disadvantages of centrifugal separators is the risk of droplet break-up due to the occurrence of strong shear layers and increased turbulence. Moreover, complete separation may not be achieved and multiple devices in series may be needed. Controllability of the process, for instance in case of slug formation, is more difficult compared to that of the gravity-based vessels. This is due to the shorter residence times in the centrifugal separator than in a gravity settler.

Swirling flow has been used successfully for other applications, such as the separation of solids from either gas [48] or liquid [11]. Liquid-liquid separation is more challenging due to the smaller density difference between the phases, high volume fraction of oil, poor coalescence and the danger of emulsion formation. Early research on hydrocyclones for liquid-liquid separation was carried out by Colman [21]. Further work on this type of separators is an active field of research, see for instance [39, 81, 99]. Dirkzwager [29] designed an axial hydrocyclone for in-line liquid-liquid separation. In an axial hydrocyclone the separated phases flow co-currently towards their respective downstream outlets. Single-phase experiments were carried out for this separator by Dirkzwager. Subsequently, Murphy et al. [78] compared these measurements with numerical results from two different computational fluid dynamics (CFD) packages. It was found that the main features of the flow were qualitatively well represented in the numerical simulations. However, large quantitative differences were observed between the numerical results mutually and between numerical results and experimental data. The in-line separator was further developed and investigated numerically by Delfos et al. [27]. This involved the design of an oil extraction outlet and the development of a computationally inexpensive numerical tool for the design of separator prototypes. The development in the present project is based on this previous work.

\subsection{Project structure and objectives}

The present project is a research project of the Institute for Sustainable Process Technology (ISPT) research project: Oil \& Gas 00-004. ISPT is a public-private partnership 
facilitating technology innovation programs in which universities, industry partners and knowledge institutes collaborate. The present project involves three universities, the University of Twente (UT), Delft University of Technology (TUD) and Wageningen University and Research Centre (WUR) and four industrial partner companies; FMC-CDS separation systems, Frames BV, Shell and Wintershall. The goal is to investigate the processes of enhanced oil-water separation by swirling flow encountered in these dense dispersions. Although cyclonic separation equipment for oil-water separation is already available on the market, there is a strong need for a better understanding of these processes. At the University of Twente the focus is on the modeling and on numerical simulations using CFD. That part of the ISPT project is discussed in this thesis. At Delft University of Technology the large-scale flow loop experiments are carried out by Laurens van Campen. At the Wageningen University Thomas Krebs studies dispersions on a smaller scale; specifically the coalescence behavior.

At the UT the CFD is utilized as a tool to obtain a good understanding of flow patterns of the swirling turbulent flow of oil-water mixtures. The CFD methodology employed uses two-phase flow models that incorporate a coalescence model for oil droplets. The aim is to understand the oil-water separation capability of devices using swirling oil-water flows as function of key parameters, such as geometry, oil properties and operating conditions. These numerical results are validated by the results of the experiments carried out at the TUD for both single-phase water flow and for oil-water flows. The effect of operational parameters on the separation efficiency and pressure drop is described. From the coalescence studies at the WUR an understanding and description of the various droplet coalescence mechanisms will be attained as well as insight how the dispersion properties depend on oil properties and surfactant concentrations.

\subsection{Outline of thesis}

In chapter two the CFD methodology used is described in some detail. This discussion includes the governing equations for single and two-phase flow, the turbulence model necessary for the closure of the Reynolds-averaged-Navier-Stokes (RANS) equations and the coupling between the equations for oil and water in the Eulerian model of two-phase flow. Subsequently, chapter three describes the design of the prototype of the oil-water separator. In addition, the experimental setup at the TUD is briefly discussed. In chapter four the results for single-phase flow through the separator are examined, including a comparison between numerical and experimental results. A similar analysis for the results obtained for the two-phase flow is presented in chapter five. The topic of chapter six is the research on the population balances, which model the evolution of the droplet size distribution (DSD) in the two-phase flow. In chapter seven conclusions are formulated and recommendations are made for further research. 
CHAPTER 2

\section{Computational fluid dynamics}

\subsection{Introduction}

The behavior of the flow is described by the Navier-Stokes equations. For a transient, incompressible flow they are given by:

$$
\begin{aligned}
\nabla \cdot \mathbf{u} & =0 \\
\frac{\partial \mathbf{u}}{\partial t}+(\mathbf{u} \cdot \nabla) \mathbf{u} & =-\frac{\nabla p}{\rho}+\nu \nabla^{2} \mathbf{u}+\mathbf{g}
\end{aligned}
$$

Here $\rho$ is the density, $p$ is the static pressure, $\mathbf{u}$ is the velocity, $\nu$ the kinematic viscosity and $\mathrm{g}$ is the gravitational acceleration. In the present study it is assumed that the temperature does not vary significantly, so that the dynamic viscosity can be considered constant and therefore $\nu=\mu / \rho$. The flow field in the centrifugal separator is complex and cannot be predicted by analytical means. Therefore, numerical simulations are used to investigate the flow. A direct numerical simulation (DNS), which reveals all details of the flow by resolving all relevant length and time scales, is simply unattainable due to the enormous computational costs. Therefore, the Reynolds averaged Navier-Stokes (RANS) equations are used. 


\subsection{Single-phase Reynolds Averaged Navier-Stokes equa- tions}

In the derivation of the RANS equations for incompressible, isothermal turbulent flows the Reynolds decomposition of the velocity and the pressure is used

$$
\begin{gathered}
\mathbf{u}=\langle\mathbf{u}\rangle+\mathbf{u}^{\prime} \\
p=\langle p\rangle+p^{\prime}
\end{gathered}
$$

Here $\langle\mathbf{u}\rangle$ and $\langle p\rangle$ are the time-averaged mean velocity vector and time-averaged pressure, respectively. Moreover, $\mathbf{u}^{\prime}$ and $p^{\prime}$ are the fluctuating velocity vector and the fluctuating pressure, respectively. The underlying assumption is that the time-scale over which the equations are averaged is much larger than the time-scale of the turbulent fluctuations, but is much smaller than the time-scale of changes of the mean flow field quantities. Substitution of equation (2.3) in equations (2.1) and (2.2) and time-averaging the result leads to the Reynolds-averaged Navier-Stokes equations

$$
\begin{aligned}
\nabla \cdot\langle\mathbf{u}\rangle & =0 \\
\frac{\partial\langle\mathbf{u}\rangle}{\partial t}+(\langle\mathbf{u}\rangle \cdot \nabla)\langle\mathbf{u}\rangle & =-\frac{\nabla\langle p\rangle}{\rho}+\nu \nabla^{2}\langle\mathbf{u}\rangle+\mathbf{g}-\nabla \cdot\left\langle\mathbf{u}^{\prime} \mathbf{u}^{\prime}\right\rangle
\end{aligned}
$$

In equation (2.5) $\left\langle\mathbf{u}^{\prime} \mathbf{u}^{\prime}\right\rangle$ are the so-called Reynolds stresses. The quantity $\rho\left\langle\mathbf{u}^{\prime} \mathbf{u}^{\prime}\right\rangle$ can be identified as the apparent stress arising from the mean momentum flux by the fluctuating velocity field [82]. The introduction of the Reynolds stresses leads to a closure problem: a model is required that expresses the Reynolds stresses in terms of the time-averaged flow field quantities. This will be discussed in section 2.3.

Regions in the flow domain of complicated topology or fine-scale solid structures, such as membranes or honeycomb flow straighteners, can be approximated as porous domains. In the porous domain the single-phase flow equations are given by

$$
\begin{aligned}
\nabla \cdot(\gamma\langle\mathbf{u}\rangle)= & 0 \\
\gamma \frac{\partial\langle\mathbf{u}\rangle}{\partial t}+\gamma(\langle\mathbf{u}\rangle \cdot \nabla)\langle\mathbf{u}\rangle= & -\gamma \frac{\nabla p}{\rho}+\gamma \nu \nabla^{2}\langle\mathbf{u}\rangle+\gamma \mathbf{g} \\
& -\gamma \nabla \cdot\left\langle\mathbf{u}^{\prime} \mathbf{u}^{\prime}\right\rangle+\frac{\gamma}{\rho} \mathbf{S}_{m}
\end{aligned}
$$

The porous region is characterized by its porosity $\gamma(\mathbf{x})$ and the momentum loss vector $S_{m}$. The porosity $\gamma$ is defined as the fraction of the volume occupied by fluid. At the interfaces between the fluid and the porous regions a jump in porosity is present. The momentum loss vector $S_{m}$ represents the inertial loss, which depends on the dynamic pressure, that is the square of the fluid velocity

$$
\mathbf{S}_{m}=-\mathbf{K}_{\text {loss }} \frac{\rho}{2}|\langle\mathbf{u}\rangle|\langle\mathbf{u}\rangle
$$

Here $\mathbf{K}_{\text {loss }}$ is a tensor of loss coefficients. 


\subsection{Turbulence modeling}

The use of eddy-viscosity models to express the Reynolds stresses in terms of the mean quantities is not well suited for swirling flow [82]. The skewness of the flow violates the eddy-viscosity assumption that the shear stress and the velocity gradient have the same direction, as pointed out by Kitoh [57]. Various sources of turbulence, for example strain rates, are not represented by the eddy viscosity models. Moreover, the assumption of isotropic turbulence overstates the shear stresses and the radial diffusion of momentum [78].

Therefore a Reynolds stress model (RSM) is used, in which the six independent Reynolds stresses $\left\langle\mathbf{u}^{\prime} \mathbf{u}^{\prime}\right\rangle$ are provided by transport equations. One of the advantages of this model is that the production terms of the Reynolds stresses can be represented exactly. Therefore, the strain rates associated with streamline curvature and flow skewness, both occurring in swirling flow, are incorporated in the terms representing production of turbulence [45]. Moreover, anisotropic behavior of the turbulent flow can be accounted for by the use of separate transport equations for each of the Reynolds stresses.

In the present research the Reynolds stress model SSG of Speziale, Sarkar and Gatski is used [105]. Contrary to other models, this model uses a quadratic pressure-strain relation. This will be explained in more detail below. The SSG RSM is recommended for swirling flows, e.g. by Cullivan et al. [25] and Chen et al. [18]. To close the SSG model, a seventh transport equation for the turbulent dissipation rate $\epsilon$ is included.

\subsubsection{Reynolds stress transport equation}

By subtracting the Reynolds averaged Navier-Stokes equations (2.5) from the NavierStokes equations, equation (2.2), a transport equation for the velocity fluctuation $u_{j}^{\prime}$ is obtained

$$
\frac{\partial u_{j}^{\prime}}{\partial t}+u_{k} \frac{\partial u_{j}^{\prime}}{\partial x_{k}}=-u_{k}^{\prime} \frac{\partial\left\langle u_{j}\right\rangle}{\partial x_{k}}-\frac{1}{\rho} \frac{\partial p^{\prime}}{\partial x_{j}}+\nu \frac{\partial^{2} u_{j}^{\prime}}{\partial x_{k} \partial x_{k}}+\frac{\partial\left\langle u_{k}^{\prime} u_{j}^{\prime}\right\rangle}{\partial x_{k}}
$$

Here the notation of summation over re-occurring indices is used. Employing the relation

$$
\left\langle u_{j}^{\prime}\left(\frac{\partial u_{i}^{\prime}}{\partial t}+u_{k} \frac{\partial u_{i}^{\prime}}{\partial x_{k}}\right)+u_{i}^{\prime}\left(\frac{\partial u_{j}^{\prime}}{\partial t}+u_{k} \frac{\partial u_{j}^{\prime}}{\partial x_{k}}\right)\right\rangle=\frac{\partial\left\langle u_{i}^{\prime} u_{j}^{\prime}\right\rangle}{\partial t}+\left\langle u_{k}\right\rangle \frac{\partial\left\langle u_{i}^{\prime} u_{j}^{\prime}\right\rangle}{\partial x_{k}}+\frac{\partial\left\langle u_{i}^{\prime} u_{j}^{\prime} u_{k}^{\prime}\right\rangle}{\partial x_{k}}
$$

a transport equation for the Reynolds stresses can be derived

$$
\frac{\partial\left\langle u_{i}^{\prime} u_{j}\right\rangle}{\partial t}+\left\langle u_{k}\right\rangle \frac{\partial\left\langle u_{i}^{\prime} u_{j}^{\prime}\right\rangle}{\partial x_{k}}+\frac{\partial\left\langle u_{i}^{\prime} u_{j}^{\prime} u_{k}^{\prime}\right\rangle}{\partial x_{k}}=P_{i j}+\Pi_{i j}-\epsilon_{i j}+\nu \frac{\partial^{2}\left\langle u_{i}^{\prime} u_{j}^{\prime}\right\rangle}{\partial x_{k} \partial x_{k}}
$$

Here $P_{i j}$ is the production tensor which gives the Reynolds stresses generated by the gradients of the mean flow velocity. It transfers kinetic energy from the mean velocity field to the fluctuation velocity field. The production tensor is given by

$$
P_{i j}=-\left\langle u_{i}^{\prime} u_{k}^{\prime}\right\rangle \frac{\partial\left\langle u_{j}\right\rangle}{\partial x_{k}}-\left\langle u_{j}^{\prime} u_{k}^{\prime}\right\rangle \frac{\partial\left\langle u_{i}\right\rangle}{\partial x_{k}}
$$


The $\Pi_{i j}$ term is the velocity-pressure gradient tensor. The effect of the fluctuating pressure is to redistribute the energy among the Reynolds stress components. The velocity-pressure gradient tensor is given by

$$
\Pi_{i j}=-\frac{1}{\rho}\left\langle u_{i}^{\prime} \frac{\partial p^{\prime}}{\partial x_{j}}+u_{j}^{\prime} \frac{\partial p^{\prime}}{\partial x_{i}}\right\rangle
$$

The dissipation tensor $\epsilon_{i j}$ is given by

$$
\epsilon_{i j}=2 \nu\left\langle\frac{\partial u_{i}^{\prime}}{\partial x_{k}} \frac{\partial u_{j}^{\prime}}{\partial x_{k}}\right\rangle
$$

Further insight can be gained when $\Pi_{i j}$ is decomposed into

$$
\Pi_{i j}=R_{i j}-\frac{\partial T_{i j k}^{(p)}}{\partial x_{k}}
$$

Here $R_{i j}$ is the pressure-strain tensor, which redistributes the energy among the Reynolds stresses. It is given by

$$
R_{i j}=\left\langle\frac{p^{\prime}}{\rho}\left(\frac{\partial u_{i}^{\prime}}{\partial x_{j}}+\frac{\partial u_{j}^{\prime}}{\partial x_{i}}\right)\right\rangle
$$

The pressure transport tensor $T_{k i j}^{(p)}$ is given by

$$
T_{k i j}^{(p)}=\frac{1}{\rho}\left\langle u_{i}^{\prime} p^{\prime}\right\rangle \delta_{j k}+\frac{1}{\rho}\left\langle u_{j}^{\prime} p^{\prime}\right\rangle \delta_{i k}
$$

Here $\delta_{i j}$ is the Kronecker delta. It should be noted that this decomposition is not unique and other options are available. Further simplification can be achieved by grouping the pressure transport, the turbulent convection and the viscous diffusion tensors in the Reynolds stress flux tensor $T_{k i j}$

$$
T_{k i j}=T_{k i j}^{(u)}+T_{k i j}^{(p)}+T_{k i j}^{(\nu)}
$$

with the turbulent convection tensor $T_{i j k}^{(u)}$

$$
T_{k i j}^{(u)}=\left\langle u_{i}^{\prime} u_{j}^{\prime} u_{k}^{\prime}\right\rangle
$$

and the viscous diffusion tensor $T_{k i j}^{(\nu)}$

$$
T_{k i j}^{(\nu)}=-\nu \frac{\partial\left\langle u_{i}^{\prime} u_{j}^{\prime}\right\rangle}{\partial x_{k}}
$$

This yields the exact transport equation for the Reynolds stresses for an incompressible, isothermal turbulent flow

$$
\frac{\partial\left\langle u_{i}^{\prime} u_{j}\right\rangle}{\partial t}+\left\langle u_{k}\right\rangle \frac{\partial\left\langle u_{i}^{\prime} u_{j}^{\prime}\right\rangle}{\partial x_{k}}+\frac{\partial T_{i j k}}{\partial x_{k}}=P_{i j}+R_{i j}-\epsilon_{i j}
$$

The term representing convection by mean flow and the production term consist of known quantities within the framework of the RANS equations, however, the other terms have to be modeled 


\section{Reynolds stress closure}

Currently, in most Reynolds stress models the terms $T_{k i j}^{(u)}$ and $T_{k i j}^{(p)}$ are combined into $T_{k i j}^{\prime}$ and are subsequently modeled as one term [82]. Here the gradient-diffusion model of Shir [101] is used

$$
T_{k i j}^{(\nu)}+T_{k i j}^{\prime}=-\left(\nu+\frac{2}{3} C_{s} \frac{k^{2}}{\epsilon}\right) \frac{\partial\left\langle u_{i}^{\prime} u_{j}^{\prime}\right\rangle}{\partial x_{k}}
$$

Here $C_{s}$ is a constant and $k$ is the turbulent kinetic energy given by $k=\frac{1}{2} u_{i}^{\prime} u_{i}^{\prime}$.

The pressure strain tensor $R_{i j}$ is of crucial importance and its modeling has been subject to extensive research. Insight into the various mechanisms contributing to the pressurestrain tensor can be gained by examining the fluctuating pressure $p^{\prime}$. For incompressible flows taking the divergence of the Navier-Stokes equations gives a Poisson's equation for the pressure $p$. Reynolds decomposition can be applied to find an expression for the Reynolds averaged pressure $\langle p\rangle$. The latter can be subtracted from the former to find the following equation for the fluctuating pressure $p^{\prime}$

$$
\frac{1}{\rho} \nabla^{2} p^{\prime}=-2 \frac{\partial\left\langle u_{i}\right\rangle}{\partial x_{j}} \frac{\partial u_{j}^{\prime}}{\partial x_{i}}-\frac{\partial^{2}}{\partial x_{i} \partial x_{j}}\left(u_{i}^{\prime} u_{j}^{\prime}-\left\langle u_{i}^{\prime} u_{j}^{\prime}\right\rangle\right)
$$

Therefore the fluctuating pressure $p^{\prime}$ can be split into three contributions

$$
p^{\prime}=p^{(s)}+p^{(r)}+p^{(h)}
$$

Here the superscripts $s, r$ and $h$ stand for slow, rapid and harmonic, respectively. The slow pressure $p^{(s)}$ satisfies

$$
\frac{1}{\rho} \nabla^{2} p^{(s)}=-\frac{\partial^{2}}{\partial x_{i} \partial x_{j}}\left(u_{i}^{\prime} u_{j}^{\prime}-\left\langle u_{i}^{\prime} u_{j}^{\prime}\right\rangle\right)
$$

Unlike the slow pressure $p^{(s)}$, the rapid pressure $p^{(r)}$ responds directly to changes in the mean velocity gradients. It satisfies

$$
\frac{1}{\rho} \nabla^{2} p^{(r)}=-2 \frac{\partial\left\langle u_{i}\right\rangle}{\partial x_{j}} \frac{\partial u_{j}^{\prime}}{\partial x_{i}}
$$

Finally the harmonic pressure $p^{(h)}$ satisfies

$$
\frac{1}{\rho} \nabla^{2} p^{(h)}=0
$$

In the same manner the pressure-strain tensor can be split into the three contributions, for instance by replacing $p^{\prime}$ in equation $(2.17)$ by $p^{(s)}$. The harmonic contribution $R_{i j}^{(h)}$ is often neglected in the modeling since it is assumed to be much smaller than the other two contributions and is insignificant away from the wall [72]. In decaying homogeneous anisotropic turbulence $R_{i j}^{(s)}$ is the only non-zero term in the pressure-strain tensor and will 
act to make the turbulence more isotropic. Subsequently this type of turbulent flow has been used extensively for the modeling of this slow pressure or return-to-isotropy term. Often the model by Rotta [89] is used

$$
R_{i j}^{(s)}=-C_{R} \epsilon a_{i j}
$$

Here the constant $C_{R}=1.8$ and $a_{i j}$ is the normalized anisotropy tensor defined as

$$
a_{i j}=\frac{\left\langle u_{i}^{\prime} u_{j}^{\prime}\right\rangle}{k}-\frac{2}{3} \delta_{i j}
$$

However, for swirling flow the non-linear return-to-isotropy modeling from the SSG model [105] performs better, therefore it is used in the present work

$$
R_{i j}^{(s)}=-\epsilon\left[C_{s 1} a_{i j}+C_{s 2}\left(a_{i k} a_{k j}-\frac{1}{3} a_{l m} a_{l m} \delta_{i j}\right)\right]
$$

Here the constants $C_{s 1}=1.7$ and $C_{s 2}=-1.05$. Evidently, this term is quadratic in the anisotropy tensor. The rapid pressure-strain tensor in the SSG model is given by

$$
\begin{aligned}
R_{i j}^{(r)}= & -C_{r 1} \frac{P}{\rho} a_{i j}+C_{r 2} k S_{i j} \\
& -C_{r 3} k S_{i j} \sqrt{a_{l m} a_{l m}} \\
& +C_{r 4} k\left(a_{i k} S_{j k}+a_{j k} S_{i k}-2 / 3 a_{k l} S_{k l} \delta_{i j}\right) \\
& +C_{r 5} k\left(a_{i k} \Omega_{j k}+a_{j k} \Omega_{i k}\right)
\end{aligned}
$$

here $P$ is the production of turbulent kinetic energy

$$
P=-\frac{1}{2} P_{k k}=-\left\langle u_{k}^{\prime} u_{l}^{\prime}\right\rangle \frac{\partial\left\langle u_{k}\right\rangle}{\partial x_{l}}
$$

$S_{i j}$ is the mean strain rate tensor, defined by

$$
S_{i j}=\frac{1}{2}\left(\frac{\partial\left\langle u_{i}\right\rangle}{\partial x_{j}}+\frac{\partial\left\langle u_{j}\right\rangle}{\partial x_{i}}\right)
$$

and $\Omega_{i j}$ is the mean vorticity tensor, defined by

$$
\Omega_{i j}=\frac{1}{2}\left(\frac{\partial\left\langle u_{i}\right\rangle}{\partial x_{j}}-\frac{\partial\left\langle u_{j}\right\rangle}{\partial x_{i}}\right)
$$

The constants of the pressure strain tensor $R_{i j}$ in SSG model are given in table 2.1. The dissipation tensor $\epsilon_{i j}$ is modeled as

$$
\epsilon_{i j}=\frac{2}{3} \delta_{i j} \epsilon
$$




\begin{tabular}{|c|c||c|c|c|c|c|}
\hline$C_{s 1}$ & $C_{s 2}$ & $C_{r 1}$ & $C_{r 2}$ & $C_{r 3}$ & $C_{r 4}$ & $C_{r 5}$ \\
\hline \hline 1.7 & -1.05 & 0.9 & 0.8 & 0.65 & 0.625 & 0.2 \\
\hline
\end{tabular}

Table 2.1: Constants of rapid pressure tensor $R_{i j}^{(r)}$ in the SSG model.

Here $\epsilon$ is the dissipation rate of turbulent kinetic energy or simply dissipation. For flows at high Reynolds numbers equation (2.36) is valid due to local isotropy [82]. A separate transport equation for $\epsilon$ is part of the turbulence modeling. This equation is given by

$$
\frac{\partial \epsilon}{\partial t}+\left\langle u_{k}\right\rangle \frac{\partial \epsilon}{\partial x_{k}}=\frac{\partial}{\partial x_{k}}\left(C_{R S} \frac{k^{2}}{\epsilon} \frac{\partial \epsilon}{\partial x_{k}}\right)+\nu \frac{\partial^{2} \epsilon}{\partial x_{k} \partial x_{k}}+C_{\epsilon 1} \frac{P \epsilon}{k}-C_{\epsilon 2} \frac{\epsilon^{2}}{k}
$$

The constants $C_{\epsilon 1}=1.45, C_{\epsilon 2}=1.83$ and $C_{R S}=0.066$. For more information on the dissipation transport equation the reader is referred to Hanjalic and Launder [46]. Below the summation convention is no longer used.

\subsubsection{Wall functions}

To accurately describe the flow near the wall a very fine mesh is required in order to resolve the steep gradients in the velocity close to the wall. Moreover, damping functions must be added to the turbulence modeling to ensure its validity in the viscous sub-layer. For threedimensional industrial flow problems the former requirement leads to large computational times and the latter increases the complexity of the model. Both issues can be avoided by the use of wall functions. These wall functions employ the log-law

$$
\left\langle u_{1}\right\rangle=u_{\tau}\left(\frac{1}{\kappa} \ln \left(y^{+}\right)+B\right)
$$

Here $\left\langle u_{1}\right\rangle$ is the velocity tangential to the surface, $\kappa=0.41$ and $B=5.2$ [82]. Furthermore, $u_{\tau}$ is the so-called friction velocity

$$
u_{\tau}=\sqrt{\tau_{w} / \rho}
$$

The wall shear stress is denoted by $\tau_{w}$. The dimensionless wall distance $y^{+}$is given by

$$
y^{+}=\frac{y u_{\tau}}{\nu}
$$

Here $y$ is the distance normal to the wall. For flow parallel to the wall, equation (2.38) gives the relation between wall shear stress and velocity in the log-layer. This empirical relation can be used to bridge the viscosity-affected layer very close to the wall, the socalled viscous sublayer in which $\left\langle u_{1}\right\rangle=u_{\tau} y^{+}$, by applying the boundary condition at a distance away from the wall.

However, equation (2.38) becomes singular when the wall shear stress approaches zero. 
Therefore, the friction velocity $u_{\tau}$ in the natural logarithm is replaced with a more robust velocity scale; the nominal friction velocity $u_{\tau}^{*}$

$$
u_{\tau}^{*}=C_{\mu}^{1 / 4} k^{1 / 2}
$$

Even when the wall shear stress is zero $u_{\tau}^{*}$ will be non-zero. The derivation of the nominal friction velocity is based on the two-equation turbulence modeling, for which the wall function approach was initially developed. In a two-dimensional boundary layer, the momentum equation of the RANS equations for steady flow with a two-equation turbulence model can be approximated as

$$
\left\langle u_{1}\right\rangle \frac{\partial\left\langle u_{1}\right\rangle}{\partial x_{1}}+\left\langle u_{2}\right\rangle \frac{\partial\left\langle u_{1}\right\rangle}{\partial x_{2}}=-\frac{1}{\rho} \frac{\partial\langle p\rangle}{\partial x_{1}}+\frac{\partial}{\partial x_{2}}\left[\left(\nu+\nu_{t}\right) \frac{\partial\left\langle u_{1}\right\rangle}{\partial x_{2}}\right]
$$

The subscripts 1 and 2 refer to the tangential and the normal direction with respect to the wall, respectively. The kinematic eddy viscosity $\nu_{t}$ is defined as

$$
\nu_{t}=C_{\mu} \frac{k^{2}}{\epsilon}
$$

The empirical constant $C_{\mu}=0.09$ is used in two-equation turbulence modeling. By rewriting the velocity components in a series expansion Wilcox [115] shows that, to leading order, in the log-layer the convective term and the pressure gradient are small compared to the viscous term. Therefore, the equation can be further approximated as

$$
\frac{\partial}{\partial x_{2}}\left[\left(\nu+\nu_{t}\right) \frac{\partial\left\langle u_{1}\right\rangle}{\partial x_{2}}\right]=0
$$

Integrating and realizing that at $x_{2}=0$ the term between the brackets multiplied by the density is equal to the wall shear stress, yields

$$
\left(\nu+\nu_{t}\right) \frac{\partial\left\langle u_{1}\right\rangle}{\partial x_{2}}=\frac{\tau_{w}}{\rho}
$$

Further away from the wall, that is for larger $x_{2}$, the molecular kinematic viscosity $\nu$ can be neglected compared to the turbulent viscosity $\nu_{t}$. Also, the right hand side is by definition equal to $u_{\tau}^{2}$

$$
u_{\tau}^{2}=\nu_{t} \frac{\partial\left\langle u_{1}\right\rangle}{\partial x_{2}}
$$

Also, according to the turbulent-viscosity hypothesis the relation between the Reynolds stress and the velocity gradient for simple shear is

$$
\left\langle u_{1}^{\prime} u_{2}^{\prime}\right\rangle=-\nu_{t} \frac{\partial\left\langle u_{1}\right\rangle}{\partial x_{2}}
$$

Equating the previous two equations gives

$$
\left\langle u_{1}^{\prime} u_{2}^{\prime}\right\rangle=-u_{\tau}^{2}
$$


Equation (2.47) can be rewritten by substituting equation (2.43), multiplying both sides by $\left\langle u_{1}^{\prime} u_{2}^{\prime}\right\rangle$ and applying equation (2.33) for simple shear. This yields

$$
\left\langle u_{1}^{\prime} u_{2}^{\prime}\right\rangle^{2}=C_{\mu} k^{2} \frac{P}{\epsilon}
$$

By assuming local equilibrium, that is $P=\epsilon$, it follows that indeed a velocity scale equivalent to $u_{\tau}$ can be found

$$
u_{\tau}^{*}=\sqrt{\left|\left\langle u_{1}^{\prime} u_{2}^{\prime}\right\rangle\right|}=C_{\mu}^{1 / 4} k^{1 / 2}
$$

In addition to boundary conditions for the momentum equations, wall functions have to be provided for the Reynolds stresses and the dissipation. In the $k-\epsilon$ turbulence models the turbulent kinetic energy $k$ has a zero-gradient condition implemented at the first node off the wall. The wall functions used for the RSM are straightforward extensions of the original methods for two-equation models and therefore the Reynolds stresses also have a zero-gradient at the wall. This is consistent with the notion that there is no diffusion of kinetic energy to the wall. In the two-equation turbulence model, the equation for the dissipation is given by

$$
\frac{\partial \epsilon}{\partial t}+(\langle\mathbf{u}\rangle . \nabla) \epsilon=\nabla .\left[\left(\nu+\frac{\nu_{t}}{\sigma_{\epsilon}}\right) \nabla \epsilon\right]+C_{\epsilon 1} \frac{P \epsilon}{k}-C_{\epsilon 2} \frac{\epsilon^{2}}{k}
$$

Here $\sigma_{\epsilon}$ is a constant. In a fully-developed flow, the flow variables only depend on $x_{2}$. Furthermore, it is again assumed that the production $P$ is equal to the dissipation $\epsilon$; that the kinematic viscosity $\nu$ is small compared to the kinematic turbulent viscosity $\nu_{t}$ in the log-layer and that the convective term is small compared to the viscous term [115]. The dissipation equation then simplifies to

$$
\frac{\partial}{\partial x_{2}}\left(\frac{\nu_{t}}{\sigma_{\epsilon}} \frac{\partial \epsilon}{\partial x_{2}}\right)-\left(C_{\epsilon 2}-C_{\epsilon 1}\right) \frac{\epsilon^{2}}{k}=0
$$

This equation is satisfied by

$$
\epsilon=\frac{u_{\tau}^{* 3}}{\kappa y}=\frac{C_{\mu}^{3 / 4} k^{3 / 4}}{\kappa y}
$$

The constants are related by

$$
\kappa^{2}=\sigma_{\epsilon} C_{\mu}^{1 / 2}\left(C_{\epsilon 2}-C_{\epsilon 1}\right)
$$

In the discrete formulation $x_{2}$ is replaced with $\Delta x_{2}$, which is the distance between the first node and the wall.

A problem arises when the mesh is refined and the location of the first node off the wall is no longer in the log-layer but in the viscous sublayer. For this situation the equations of the preceding section are no longer valid. The transition from log-layer to viscous sublayer is often taken as the point where the linear velocity profile of the viscous sublayer and the logarithmic velocity profile of the log-layer intersect, that is at $y^{+}=11.06$. Therefore, 
a feature is used in which the $y^{+}$used in the calculation is not allowed to drop below this value. In effect, the log-layer is pulled towards the wall by the wall functions for $y^{+}$ $<11.06$. Evidently, it would be best if the $y^{+}$found in the computation does not drop below 11.06 .

Wall functions assume that the velocity profile follows the log-law, equation (2.38), and therefore these functions fail if the velocity profile deviates from that. This situation is encountered in separating and re-attaching flows, rotating walls, flows with strong pressure gradients or when there is locally no equilibrium between turbulent production and dissipation [45]. However, measurements from Kitoh [57] of moderately swirling pipe flow agree very well with the log-law for $y^{+}<100$. In addition, Murphy [78] concludes that overall his numerical simulations predicted the near-wall behavior quite well and thereby confirming the applicability of wall functions for strongly swirling flows. 


\subsection{Two-fluid model}

Two-phase flow is characterized by a complex topology with countless, continually changing interfaces between the two fluids. Resolving all details of the flow would be even more computationally expensive than for single-phase turbulent flow. Therefore, a spatial averaging procedure is carried out leading to a set of equations for spatially averaged quantities, which still capture the essential macroscopic details of the flow for affordable computational efforts.

\subsubsection{Volume averaging procedure}

The interior of each region with two phases is fully described by the instantaneous NavierStokes equations (2.1) and (2.2) for each individual phase while interfacial boundary conditions couple the two solutions at the interface.

Let us define an arbitrary averaging volume $V$ which is independent of space and time. Say the volume is defined in a local coordinate system $\xi_{1}, \xi_{2}$ and $\xi_{3}$, whose axes are parallel to the $\mathrm{x}, \mathrm{y}$ and $\mathrm{z}$-directions, respectively, and which has its origin at location $\mathrm{x}$. An example of such a volume is given in figure 2.1. The interest lies in obtaining averaged equations for phase $k$, this could be any of the two fluids. Since phase $k$ is not present everywhere, a phase indicator function $\gamma_{k}$ is defined to filter out the other phase.

$$
\gamma_{k}(\mathbf{x}, t)= \begin{cases}1, & \text { if phase } \mathrm{k} \text { is present at }(\mathbf{x}, t) \\ 0, & \text { otherwise }\end{cases}
$$

Now the volume average of a variable $q_{k}$ pertaining to phase $\mathrm{k}$ is given by

$$
\left\langle q_{k}\right\rangle_{V}=\frac{1}{V} \int_{V} q_{k}(\mathbf{x}+\boldsymbol{\xi}, t) \gamma_{k}(\mathbf{x}+\boldsymbol{\xi}, t) d v_{\xi}
$$

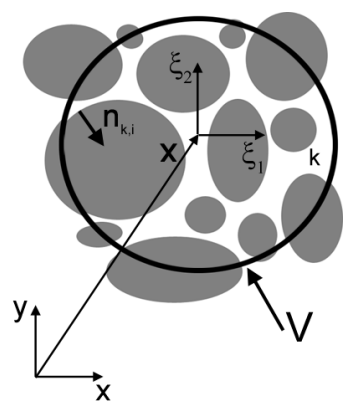

Figure 2.1: Sketch of two-dimensional averaging volume $V$ in a two-phase flow region with local coordinate system $\boldsymbol{\xi}$. Origin of volume $V$ is indicated by vector $\mathbf{x}$. The continuous fluid (white) is defined as phase $k, \mathbf{n}_{\mathbf{k}, \mathbf{i}}$ is the outward normal of phase $k$ at the interface. 
Some useful mathematical relations for the derivation of the governing equations are now discussed. The phase indicator function $\gamma_{k}(\mathbf{x}, t)$ can be seen as a sum of Heaviside functions, the derivative of which are Dirac delta functions [43]. Therefore, the gradient of $\gamma_{k}$ is given by

$$
\nabla \gamma_{k}(\mathbf{x}, t)=-\mathbf{n}_{k, i} \delta\left(\mathbf{x}-\mathbf{x}_{k, i}\right)
$$

Here $\mathbf{x}_{k, i}$ is the position vector of the interface between phase $\mathrm{k}$ and the other phase. Furthermore, $\mathbf{n}_{k, i}$ is the outward normal of phase $k$ at the interface. The gradient of $\gamma_{k}(\mathbf{x}, \mathbf{t})$ is only non-zero at the interface. Furthermore, the total derivative of the phase indicator function $\gamma_{k}(\mathbf{x}, t)$ with respect to time is given by

$$
\frac{d \gamma_{k}}{d t}=\frac{\partial \gamma_{k}}{\partial t}+\frac{\partial \gamma_{k}}{\partial x} \frac{\partial x}{\partial t}+\frac{\partial \gamma_{k}}{\partial y} \frac{\partial y}{\partial t}+\frac{\partial \gamma_{k}}{\partial z} \frac{\partial z}{\partial t}
$$

This can be rewritten into

$$
\frac{d \gamma_{k}}{d t}=\frac{\partial \gamma_{k}}{\partial t}+\mathbf{u}_{k, i} \cdot \nabla \gamma_{k}
$$

Here $\mathbf{u}_{k, i}$ is velocity at the interface. In this work mass transfer will be assumed to be absent, therefore the phase indicator $\gamma_{k}$ will move with the flow and an observer moving with the interface will not see a change in $\gamma_{k}$, therefore the total derivative is zero. This leads to the following relation

$$
\frac{\partial \gamma_{k}}{\partial t}=-\mathbf{u}_{k, i} \cdot \nabla \gamma_{k}
$$

A fundamental property of the $\delta$-function is that an integral, whose integrand is a Dirac $\delta$-function multiplied by some other function, for instance $q_{k} \mathbf{n}_{k, i}$, is that other function evaluated at the singular points of the $\delta$-function [56].

$$
\frac{1}{V} \int_{V} q_{k}(\mathbf{x}+\boldsymbol{\xi}, t) \mathbf{n}_{k, i} \delta\left(\mathbf{x}+\boldsymbol{\xi}-\mathbf{x}_{k, i}, t\right) d v_{\xi}=\frac{1}{V} \int_{A_{k, i}} q_{k}(\mathbf{x}+\boldsymbol{\xi}, t) \mathbf{n}_{k, i} d A
$$

The interface surface $A_{k, i}$ is defined by $\mathbf{x}=\mathbf{x}_{k, i}$.

Besides the averaged quantity defined in equation (2.56), one is often interested in the quantity

$$
\left\langle q_{k}\right\rangle=\frac{1}{V_{k}} \int_{V} q_{k}(\mathbf{x}+\boldsymbol{\xi}, t) \gamma_{k}(\mathbf{x}+\boldsymbol{\xi}, t) d v_{\xi}=\frac{1}{V_{k}} \int_{V_{k}} q_{k}(\mathbf{x}+\boldsymbol{\xi}, t) d v_{\xi}
$$

which gives the average of the quantity $q_{k}$ while only taking into account the volume that phase $k$ occupies. When comparing equations (2.56) and (2.62) the following relation can be found

$$
\left\langle q_{k}\right\rangle_{V}=\alpha_{k}\left\langle q_{k}\right\rangle
$$

In which $\alpha_{k}$ is readily identified as the volume fraction of phase $k$ :

$$
\alpha_{k}=\frac{V_{k}}{V}=\frac{1}{V} \int_{V} \gamma_{k}(\mathbf{x}+\boldsymbol{\xi}, t) d v_{\xi}
$$


Equations (2.57), (2.60), (2.61) and (2.63) will be applied in the averaging procedure. For convenience the dependance on space and time of variables is from now on no longer explicitly mentioned.

Now multiplying the instantaneous continuity equation for phase $k$

$$
\nabla \cdot \mathbf{u}_{k}=0
$$

by the phase indicator function $\gamma_{k}$ and integrating over volume $V$ leads to

$$
\frac{1}{V} \int_{V} \gamma_{k} \nabla \cdot \mathbf{u}_{k} d v_{\xi}=0
$$

This expression will lead to the average of the divergence of velocity $\mathbf{u}_{k}$, while the interest is in the divergence of the averaged velocity $\left\langle\mathbf{u}_{k}\right\rangle$. Therefore, the chain rule of differentiation is employed to rewrite equation (2.66) as

$$
\frac{1}{V} \int_{V} \nabla \cdot\left(\mathbf{u}_{k} \gamma_{k}\right) d v_{\xi}-\frac{1}{V} \int_{V} \mathbf{u}_{k} \cdot \nabla \gamma_{k} d v_{\xi}=0
$$

Substitution of equation (2.60) and realizing that the space and time-derivatives can be moved outside the integrals since this volume $V$ does not depend on space $\mathrm{x}$ or time $\mathrm{t}$, leads to

$$
\nabla \cdot\left(\frac{1}{V} \int_{V}\left(\mathbf{u}_{k} \gamma_{k}\right) d v_{\xi}\right)+\frac{\partial}{\partial t}\left(\frac{1}{V} \int_{V} \gamma_{k} d v_{\xi}\right)=0
$$

Using equations (2.56), (2.63) and (2.64) and rearranging, the final expression for the continuity equation for phase $k$ is found:

$$
\frac{\partial \alpha_{k}}{\partial t}+\nabla \cdot\left(\alpha_{k}\left\langle\mathbf{u}_{k}\right\rangle\right)=0
$$

A similar procedure is followed for the instantaneous momentum equation for phase $k$ given by

$$
\frac{\partial \mathbf{u}_{k}}{\partial t}+\nabla \cdot\left(\mathbf{u}_{k} \mathbf{u}_{k}\right)=-\frac{1}{\rho_{k}} \nabla p_{k}+\nu_{k} \nabla \cdot\left(\nabla \mathbf{u}_{k}+\left(\nabla \mathbf{u}_{k}\right)^{T}\right)+\mathbf{g}
$$

A constant kinematic viscosity for phase $k$ is assumed here. This leads to

$$
\begin{aligned}
\frac{1}{V} \int_{V} \frac{\partial \mathbf{u}_{k}}{\partial t} \gamma_{k} d v_{\xi}+\frac{1}{V} \int_{V} \nabla \cdot\left(\mathbf{u}_{k} \mathbf{u}_{k}\right) \gamma_{k} d v_{\xi}= & -\frac{1}{\rho_{k} V} \int_{V} \nabla p_{k} \gamma_{k} d v_{\xi} \\
& +\frac{1}{V} \int_{V} \nu_{k} \nabla \cdot\left(\nabla \mathbf{u}_{k}+\left(\nabla \mathbf{u}_{k}\right)^{T}\right) \gamma_{k} d v_{\xi} \\
& +\frac{1}{V} \int_{V} \mathbf{g} \gamma_{k} d v_{\xi}
\end{aligned}
$$


By applying the chain rule of differentiation on the first term on the left-hand side and realizing that the volume does not depend on time, the result is

$$
\frac{1}{V} \int_{V} \frac{\partial \mathbf{u}_{k}}{\partial t} \gamma_{k} d v_{\xi}=\frac{\partial}{\partial t}\left(\frac{1}{V} \int_{V} \mathbf{u}_{k} \gamma_{k} d v_{\xi}\right)-\frac{1}{V} \int_{V} \mathbf{u}_{k} \frac{\partial \gamma_{k}}{\partial t} d v_{\xi}
$$

Now using equations (2.60) and (2.57) the following is attained

$$
\frac{1}{V} \int_{V} \frac{\partial \mathbf{u}_{k}}{\partial t} \gamma_{k} d v_{\xi}=\frac{\partial}{\partial t}\left(\frac{1}{V} \int_{V} \mathbf{u}_{k} \gamma_{k} d v_{\xi}\right)-\frac{1}{V} \int_{V} \mathbf{u}_{k}\left(\mathbf{u}_{k} \cdot \mathbf{n}_{k, i}\right) \delta\left(\mathbf{x}-\mathbf{x}_{k, i}\right) d v_{\xi}
$$

Using equations (2.56) and (2.63) to rewrite the first term on the right-hand side and using (2.61) to arrive at a surface integral for the second term, the final result is

$$
\frac{1}{V} \int_{V} \frac{\partial \mathbf{u}_{k}}{\partial t} \gamma_{k} d v_{\xi}=\frac{\partial}{\partial t}\left(\alpha_{k}\left\langle\mathbf{u}_{k}\right\rangle\right)-\frac{1}{V} \int_{A_{k, i}} \mathbf{u}_{k}\left(\mathbf{u}_{k} \cdot \mathbf{n}_{k, i}\right) d A
$$

For the other terms in equation (2.71) the following averaging procedure is used. First, the term is rewritten using the chain rule of differentiation, as was done in equation (2.67). Subsequently, equations (2.57) and (2.61) are applied, which leads to a term with a surface integral. Finally, equations (2.56) and (2.63) are used to rewrite the volume integral into the desired form:

$$
\begin{aligned}
& \frac{1}{V} \int_{V} \nabla \cdot\left(\mathbf{u}_{k} \mathbf{u}_{k}\right) \gamma_{k} d v_{\xi}= \nabla \cdot\left(\alpha_{k}\left\langle\mathbf{u}_{k} \mathbf{u}_{k}\right\rangle\right)+\frac{1}{V} \int_{A_{k, i}} \mathbf{u}_{k}\left(\mathbf{u}_{k} \cdot \mathbf{n}_{k, i}\right) d A \\
& \frac{1}{\rho_{k} V} \int_{V} \nabla p_{k} \gamma_{k} d v_{\xi}= \frac{1}{\rho_{k}} \nabla\left(\alpha_{k}\left\langle p_{k}\right\rangle\right)+\frac{1}{\rho_{k} V} \int_{A_{k, i}} p_{k} \mathbf{n}_{k, i} d A \\
& \frac{1}{V} \int_{V} \nu_{k} \nabla \cdot\left(\nabla \mathbf{u}_{k}+\left(\nabla \mathbf{u}_{k}\right)^{T}\right) \gamma_{k} d v_{\xi}=\nu_{k} \nabla \cdot\left(\alpha_{k}\left\langle\nabla \mathbf{u}_{k}+\left(\nabla \mathbf{u}_{k}\right)^{T}\right\rangle\right) \\
&+\frac{1}{V} \int_{A_{k, i}} \nu_{k}\left(\nabla \mathbf{u}_{k}+\left(\nabla \mathbf{u}_{k}\right)^{T}\right) \cdot \mathbf{n}_{k, i} d A \\
& \frac{1}{V} \int_{V} \mathbf{g} \gamma_{k} d v_{\xi}= \alpha_{k}\langle\mathbf{g}\rangle
\end{aligned}
$$


Substitution of equations (2.74) to (2.79) and applying the Reynolds decomposition, equation (2.3) gives

$$
\begin{aligned}
\frac{\partial}{\partial t}\left(\alpha_{k}\left\langle\mathbf{u}_{k}\right\rangle\right)+\nabla \cdot\left(\alpha_{k}\left\langle\mathbf{u}_{k}\right\rangle\left\langle\mathbf{u}_{k}\right\rangle\right)= & -\frac{1}{\rho_{k}} \nabla\left(\alpha_{k}\left\langle p_{k}\right\rangle\right) \\
& +\nu_{k} \nabla \cdot\left(\alpha_{k}\left\langle\nabla \mathbf{u}_{k}+\left(\nabla \mathbf{u}_{k}\right)^{T}\right\rangle\right) \\
& +\alpha_{k}\langle\mathbf{g}\rangle-\nabla \cdot\left(\alpha_{k}\left\langle\mathbf{u}_{k}^{\prime} \mathbf{u}_{k}^{\prime}\right\rangle\right) \\
& +\frac{1}{\rho_{k} V} \int_{A_{k, i}}\left[-p_{k} \mathbf{n}_{k, i}+\mu_{k}\left(\nabla \mathbf{u}_{k}+\left(\nabla \mathbf{u}_{k}\right)^{T}\right) \cdot \mathbf{n}_{k, i}\right] d A
\end{aligned}
$$

Furthermore, the pressure term $\nabla\left(\alpha_{k}\left\langle p_{k}\right\rangle\right)$ is split into two parts

$$
\nabla\left(\alpha_{k}\left\langle p_{k}\right\rangle\right)=\alpha_{k} \nabla\left\langle p_{k}\right\rangle+\left\langle p_{k}\right\rangle \nabla \alpha_{k}
$$

The second term is grouped with the surface integral term, which is also known as the interfacial source term, and together these two terms are identified as the force acting on phase $k$. For instance, a static pressure increase in an incompressible two-phase mixture would not alter the interfacial force. However, the surface integral will change if surface $A_{k, i}$ is not a closed surface, as is the case in figure 2.1 , where some of the droplets are intersected by the averaging volume. If the interfacial force is solely formed by the surface integral this would lead to non-physical results. Yet the inclusion of the term $\left\langle p_{k}\right\rangle \nabla \alpha_{k}$, which is non-zero if the surface $A_{k, i}$ is open, cancels out the integral. Prosperetti and Tryggvason [85] argue that the product of the averaged total stress tensor and the volume fraction gradient, that is $\left[\left\langle p_{k}\right\rangle I+\mu\left\langle\nabla \mathbf{u}+(\nabla \mathbf{u})^{T}\right\rangle\right] \cdot \nabla \alpha_{k}$, should also be included in the interfacial force. However, Ishii [52] and Drew [30] only incorporate the pressure term. The latter definition is used here since it is also used in Ansys CFX 14.0. For convenience, the following definition is used

$$
\tau_{k}=\mu_{k}\left(\nabla \mathbf{u}_{k}+\left(\nabla \mathbf{u}_{k}\right)^{T}\right)
$$

It is customary to rewrite the interfacial source term by splitting both $p_{k}$ and $\tau_{k}$ in a surface averaged part and a variation with respect to that averaged part, the latter is due to turbulence and the movement of the interfaces caused by the flow $[52,31]$ :

$$
\begin{aligned}
p_{k} & =\left\langle p_{k}\right\rangle_{A_{k, i}}+p_{k}^{S^{\prime}} \\
\tau_{k} & =\left\langle\tau_{k}\right\rangle_{A_{k, i}}+\tau_{k}^{S^{\prime}}
\end{aligned}
$$


Now the interfacial source term is rewritten as

$$
\begin{aligned}
\frac{1}{V} \int_{A_{k, i}}\left[-p_{k} \mathbf{n}_{k, i}+\tau_{k} \cdot \mathbf{n}_{k, i}\right] d A= & \frac{1}{V} \int_{A_{k, i}}\left[-\left\langle p_{k}\right\rangle_{A_{k}, i} I+\left\langle\tau_{k}\right\rangle_{A_{k, i}}\right] \cdot \mathbf{n}_{k, i} d A \\
& +\frac{1}{V} \int_{A_{k, i}}\left(-p_{k}^{S^{\prime}} I+\tau_{k}^{S^{\prime}}\right) \cdot \mathbf{n}_{k, i} d A \\
= & {\left[\left\langle p_{k}\right\rangle_{A_{k}, i} I-\left\langle\tau_{k}\right\rangle_{A_{k}, i}\right] \frac{1}{V} \int_{V} \nabla \gamma_{k} d V+M_{k, i} } \\
= & \left\langle p_{k}\right\rangle_{A_{k}, i} \nabla \alpha_{k}-\left\langle\tau_{k}\right\rangle_{A_{k, i}} \nabla \alpha_{k}+M_{k, i}
\end{aligned}
$$

Here $M_{k, i}$ is the generalized interfacial drag term:

$$
M_{k, i}=\frac{1}{V} \int_{A_{k, i}}\left(-p_{k}^{S^{\prime}} I+\tau_{k}^{S^{\prime}}\right) \cdot \mathbf{n}_{k, i} d A
$$

This term accounts for the pressure and viscous stresses that work on the dispersed phase integrated over de wetted area of the dispersed phase, which we could call drag and lift. Often the term $\left\langle\tau_{k}\right\rangle_{A_{k, i}} \nabla \alpha_{k}$ is also included in the generalized interfacial drag term $[30,116]$ :

$$
M_{k, i}=\frac{1}{V} \int_{A_{k, i}}\left(-p_{k}^{S^{\prime}} I+\tau_{k}\right) \cdot \mathbf{n}_{k, i} d A
$$

This latter definition is used here, since it is also used in Ansys CFX 14.0. In the present study effects of surface tension are not taken into account in the formulation of the momentum equations of the two-fluid model and therefore

$$
\sum_{k=1}^{2}\left[\left\langle p_{k}\right\rangle_{A_{k, i}} \nabla \alpha_{k}+M_{k, i}\right]=0
$$

Also, since effects of surface tension are neglected it is reasonable to assume that the two phases share the same pressure field, that is $\left\langle p_{1}\right\rangle=\left\langle p_{2}\right\rangle=\langle p\rangle$. Therefore, since in the current work $\alpha_{1}+\alpha_{2}=1$ :

$$
\sum_{k=1}^{2}\langle p\rangle \nabla \alpha_{k}=0
$$

this is leads to

$$
\sum_{k=1}^{2} M_{k, i}=0
$$

Moreover, the pressure difference $\left(\left\langle p_{k}\right\rangle_{A_{k, i}}-\left\langle p_{k}\right\rangle\right) \nabla \alpha_{k}$ now appears in the equations. This difference is non-zero when velocities are comparable to the speed of sound, for 
example in situations in which acoustics are of importance. Otherwise this term can be neglected [30]. The final momentum equation then becomes

$$
\begin{aligned}
\frac{\partial}{\partial t}\left(\alpha_{k}\left\langle\mathbf{u}_{k}\right\rangle\right)+\nabla \cdot\left(\alpha_{k}\left\langle\mathbf{u}_{k}\right\rangle\left\langle\mathbf{u}_{k}\right\rangle\right)= & -\frac{1}{\rho_{k}} \alpha_{k} \nabla\langle p\rangle+\nu_{k} \nabla \cdot\left(\alpha_{k}\left\langle\nabla \mathbf{u}_{k}+\left(\nabla \mathbf{u}_{k}\right)^{T}\right\rangle\right) \\
& +\alpha_{k}\langle\mathbf{g}\rangle-\nabla \cdot\left(\alpha_{k}\left\langle\mathbf{u}_{k}^{\prime} \mathbf{u}_{k}^{\prime}\right\rangle\right)+\frac{1}{\rho_{k}} M_{k, i}
\end{aligned}
$$

For convenience the volume fraction of the oil is now defined as $\alpha$, that is $\alpha=\alpha_{o}$. The volume fraction of the water phase is given by $\alpha_{w}=(1-\alpha)$.

\subsubsection{Interfacial coupling}

For the derivation of the generalized interfacial drag term $M_{k, i}$ the assumption of dispersed oil droplets in water is made. In principle, all known interfacial forces such as drag force, virtual mass force, lift force, wall lubrication force, Basset history force and turbulent dispersion forces can be included in this term.

\section{Drag force}

The most important interfacial force in the drag force. The expression for the drag force acting on the oil phase is given by

$$
M_{o}=\frac{3}{4} \frac{C_{D}}{d} \rho_{w} \alpha\left|\left\langle\mathbf{u}_{w}\right\rangle-\left\langle\mathbf{u}_{o}\right\rangle\right|\left(\left\langle\mathbf{u}_{w}\right\rangle-\left\langle\mathbf{u}_{o}\right\rangle\right)
$$

Here $C_{D}$ is the drag coefficient, $d$ is the diameter of the oil droplets and the subscripts $w$ and $o$ stand for water and oil, respectively. The formulation of the drag law by Schiller

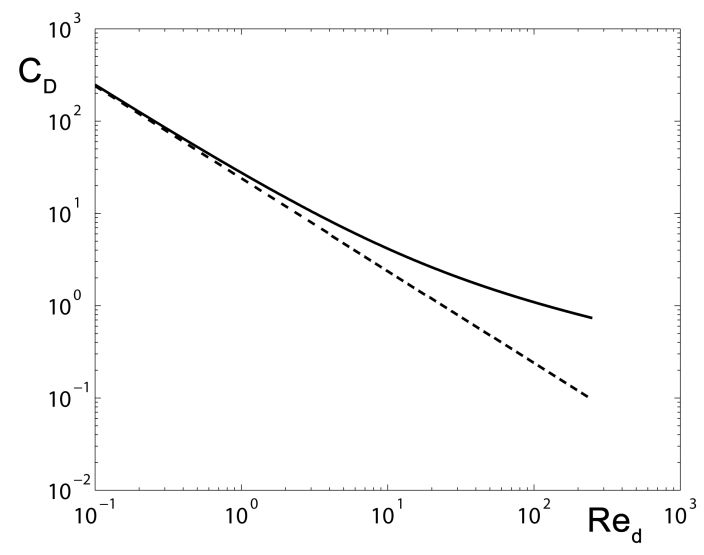

Figure 2.2: Drag coefficient $C_{D}$ as function of Reynolds number $\mathrm{Re}_{\mathrm{d}}$ as given by equation 2.92 (solid) and the one by Stokes drag: $C_{D}=24 / R e_{d}$ (dashed). 
and Naumann [97] and the one by Ishii and Zuber [53] are used in the present work. The correlation of Schiller and Naumann is defined as

$$
C_{D}=\frac{24}{\operatorname{Re}_{\mathrm{d}}}\left(1+0.15 \mathrm{Re}_{\mathrm{d}}^{0.687}\right)
$$

Here $\operatorname{Re}_{\mathrm{d}}$ is the Reynolds number based on the droplet diameter and the relative velocity:

$$
\operatorname{Re}_{\mathrm{d}}=\frac{\rho_{w}\left|\left\langle\mathbf{u}_{o}\right\rangle-\left\langle\mathbf{u}_{w}\right\rangle\right| d}{\mu_{w}}
$$

The drag coefficient $C_{D}$ as function of $\mathrm{Re}_{\mathrm{d}}$ for the formulation by Schiller and Naumann and by Stokes;

$$
C_{D}=\frac{24}{\operatorname{Re}_{\mathrm{d}}}
$$

are shown in figure 2.2. The correlation in equation (2.92) is applicable to spherical droplets for Reynolds numbers up to 1000 . However, it does not take into account the hinderance effect that other, nearby droplets will have on the movement of a droplet. The presence of a dispersed phase leads to a higher resistance to deformation of the flow field. It was shown by Einstein that the viscosity $\mu_{m}$ of a dilute mixture with small suspended solid spheres increases with increasing particle concentration [33, 34] like $\mu_{m} / \mu_{w}=1+2.5 \alpha$. Later, this result was extended to small spherical droplets by Taylor [108], taking the flow inside the droplets into account. The ratio of the viscosity of the mixture $\mu_{m}$ to that of the continuous water phase $\mu_{w}$ is now given as

$$
\frac{\mu_{m}}{\mu_{w}}=1+2.5 \alpha\left(\frac{\mu_{o}+2 / 5 \mu_{w}}{\mu_{o}+\mu_{w}}\right)
$$

The original expression of Einstein is recovered for very high viscosity $\mu_{o}$ of the dispersed oil phase. The result for solid spheres has been extended to high volume fractions of the dispersed phase by Roscoe [88]. In his derivation, Roscoe starts with very small particles suspended in a liquid. The viscosity of this mixture is readily derived by employing Einstein's expression for the mixture viscosity. Subsequently, the volume fraction is increased by adding much larger particles. Compared to the mixture with these large particles, the continuous fluid with the much smaller particles can be considered as a homogeneous mixture, of which the mixture viscosity was previously computed. By employing Einstein's expression once more the viscosity of the new mixture is obtained. By repeating this procedure, the change in viscosity with increasing volume fraction of the dispersed phase is obtained. This relation is then integrated to yield

$$
\frac{\mu_{m}}{\mu_{w}}=(1-\alpha)^{-2.5}
$$

The results of Taylor and Roscoe have been combined by Ishii and Zuber [53] who give the mixture viscosity for high volume fraction systems as

$$
\mu_{m}=\mu_{w}(1-\alpha)^{-2.5 \frac{\mu_{o}+2 / 5 \mu_{w}}{\mu_{o}+\mu_{w}}}
$$




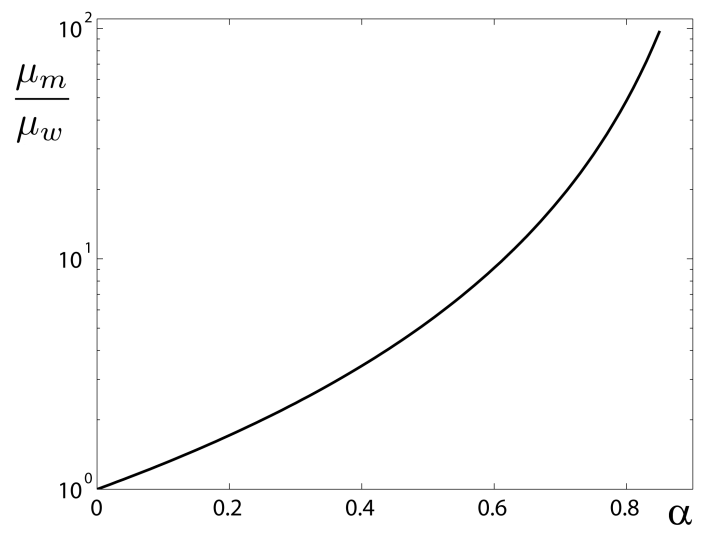

Figure 2.3: Ratio of the viscosity of the mixture $\mu_{m}$ to that of the water phase $\mu_{w}$ as given by equation (2.97). The water viscosity is that of water with $9 \mathrm{wt} \%$ salt: $\mu_{w}=1.183 \times 10^{-3}$ $\mathrm{kg} /(\mathrm{m} . \mathrm{s})$. The oil viscosity is that of Shell Morlina 10: $\mu_{o}=19.5 \times 10^{-3} \mathrm{~kg} /(\mathrm{m} . \mathrm{s})$.

The ratio of the viscosity of the mixture $\mu_{m}$ to that of the water phase $\mu_{w}$ given by equation (2.97) is shown in figure 2.3. The viscosity of water in equation (2.93) is therefore replaced by the mixture viscosity of equation (2.97) to give the following expression for the drag coefficient for dense emulsions of undistorted droplets:

$$
C_{D}=\frac{24}{\operatorname{Re}_{\mathrm{d}}}\left(1+0.15 \operatorname{Re}_{\mathrm{d}}{ }^{0.687}\right)(1-\alpha)^{-2.5 \frac{\mu_{o}+2 / 5 \mu_{w}}{\mu_{o}+\mu_{w}}}
$$

The underlying assumptions are that the dispersed phase is not deformed and the droplets do not directly interact with each other. Also, a wide size distribution was assumed in the derivation of the mixture viscosity, while the interfacial area is estimated based on a single diameter $d$. This is an internal contradiction of the model.

The drag coefficient $C_{D}$ will increase exponentially for $\alpha$ approaching unity. Therefore, the velocity difference between the oil phase and the water phase will become very small and further separation is prevented, limiting the increase in oil volume fraction. Ishii and Zuber claim their drag relation can be used for dispersed volume fractions up to 0.95 if the dispersed phase is a gas or liquid. However, in a real application it is likely that at lower oil volume fractions coalescence between the droplets will occur. At a certain point phase inversion is likely to occur and water will become the dispersed phase. However, in the current drag formulation the oil is treated as the dispersed phase, regardless of the value of the oil volume fraction.

\section{Turbulent dispersion}

The effect of turbulence on the motion of the dispersed phase is not completely captured by the drag law. The drag law only accounts for the interfacial forces arising from the 
difference between the mean velocity of the dispersed phase and that of the continuous phase. The SSG turbulence model accounts for the effect of turbulence on the mean flow field and therefore turbulence only indirectly influences the relative motion of the phases. The effect of turbulent fluctuations on motion of the dispersed phase can be incorporated by turbulent dispersion models. Their use in computing two-phase flow is not widely spread, indicating some uncertainty with regards to their validity and accuracy. Burns et al. [14] state that the term which can be interpreted as turbulent dispersion arises in the derivation of the two-phase flow equations after applying both volume and time averaging. This term contains the, in time, fluctuating oil volume fraction. However, Gosman et al. [42] employ a similar model for the turbulent dispersion while starting with the volume-averaged equations for two-phase flow. In CFX 14.0 the model by Burns et al. [14] is available. The turbulent dispersion force acting on the oil phase is given by

$$
M_{o}^{T D}=-C_{1} \frac{3}{4} \frac{C_{D}}{d} \rho_{w} \alpha\left|\left\langle\mathbf{u}_{o}\right\rangle-\left\langle\mathbf{u}_{\mathbf{w}}\right\rangle\right| \frac{\nu_{t}}{\sigma_{t}} \frac{\nabla \alpha}{\alpha(1-\alpha)}
$$

The adjustable parameter $C_{1}$ is unity by default. The turbulent Schmidt number $\sigma_{t}$ is set to 0.9. The turbulent Schmidt number is defined as the ratio of the turbulent momentum diffusivity or eddy viscosity $\nu_{t}$ and the turbulent mass diffusivity $D_{t}$ :

$$
\sigma_{t}=\frac{\nu_{t}}{D_{t}}
$$

The model assumes isotropic turbulence and so the gradient-diffusion and the turbulentviscosity hypothesis are applied in the construction of the model. It is assumed that the diffusion will take place in the direction of the gradient of the volume fraction, and that the strength of the diffusion is given by the eddy viscosity $\nu_{t}$.

\section{Virtual mass force}

When a droplet is accelerated, there will be a corresponding acceleration of the surrounding fluid. A force equal but opposite in sign to the force required for the acceleration of the surrounding fluid will act on the droplet. Since a larger force will be needed for a certain acceleration of the droplet than expected, based on the mass of the droplet, the droplet appears to have a larger mass. The effect is accounted for by the virtual mass force. The virtual mass force acting on the disperse phase per unit volume is given by:

$$
M_{o}^{A D}=\alpha \rho_{w} C_{A D}\left(\frac{D\left\langle\mathbf{u}_{o}\right\rangle}{D t}-\frac{D\left\langle\mathbf{u}_{w}\right\rangle}{D t}\right)
$$

The virtual mass constant $C_{A D}=0.5$ for spherical droplets. Its value is derived by equating the change in kinetic energy of the inviscid, incompressible, accelerated flow surrounding a spherical droplet to the work done by the added mass force [24]. The flow field surrounding the droplet is computed using potential flow. No other widely accepted correlation for $C_{A D}$ exists. As can be seen in equation (2.90), the generalized interfacial drag term $M_{k, i}$ is divided by the density of the fluid $k$. Therefore, in cases with a high density ratio of the disperse and the continuous phase, such as in bubbly flow, the virtual mass force has a significant effect on the behavior of the dispersed phase. In the current liquid-liquid application its effect will be smaller. 


\section{Lift force}

In a shear layer, the dispersed phase experiences a lateral lift force because the difference in velocity over the droplet results in a difference in the pressure distribution. This lift force leads, for instance, to non-uniform gas volume fraction distributions in vertical pipes with bubbly flow. Accurately describing the lift force is complicated and some observed trends are not completely understood [24]. Depending on the deformation of the droplet even negative lift forces due to flow separation and vortex shedding have been observed. An additional problem is that the effects on the lift force of high volume fractions of the dispersed phase are largely unknown [92]. The lift force is often assumed to be insignificant and therefore not incorporated in the numerical simulations of hydrocyclones [79].

The lift force model of Saffman-Mei [77, 93, 94] is employed in the present work. The original model by Saffman for spherical solid particles has been extended by Mei to $\mathrm{Re}_{\mathrm{d}}$ up to 100 . In the current application oil droplets are assumed to be spherical due to their small size. Also the relatively high viscosity of the oil will effectively prevent internal circulation and therefore the droplets can be approximated as solids. Due to the high drag force the relative velocity is likely to be small leading to low values of $R_{d}$ for which this model is suited. The Saffman-Mei model is given by

$$
\begin{aligned}
M_{o}^{L} & =\frac{3}{2 \pi} \frac{\sqrt{\rho_{w} \mu_{w}}}{d \sqrt{\left|\boldsymbol{\omega}_{w}\right|}} C_{l} f_{l} \rho_{w} \alpha \Delta\langle\mathbf{u}\rangle \times \boldsymbol{\omega}_{w} \\
& =\frac{3}{2 \pi} \sqrt{\frac{\mathrm{Ro}}{\mathrm{Re}_{\mathrm{d}}}} C_{l} f_{l} \rho_{w}^{2} \alpha \Delta\langle\mathbf{u}\rangle \times \boldsymbol{\omega}_{w}
\end{aligned}
$$

Here $\boldsymbol{\omega}_{w}$ is the vorticity, given by

$$
\boldsymbol{\omega}_{w}=\nabla \times\left\langle\mathbf{u}_{w}\right\rangle
$$

The velocity difference of the mean velocities is given by

$$
\Delta\langle\mathbf{u}\rangle=\left\langle\mathbf{u}_{w}\right\rangle-\left\langle\mathbf{u}_{o}\right\rangle
$$

In the present study the Rossby number Ro is defined as

$$
\operatorname{Ro}=\frac{|\Delta\langle\mathbf{u}\rangle|}{\left|\boldsymbol{\omega}_{w}\right| d}
$$

The lift coefficient $C_{l}=6.46$ and the $f_{l}$ is the correction function of Mei et al. This correction function is given by

$$
\begin{array}{lr}
f_{l}=e^{-0.1 \operatorname{Re}_{\mathrm{d}}}-\frac{0.3314}{\sqrt{2 \mathrm{Ro}}}\left(e^{-0.1 \mathrm{Re}_{\mathrm{d}}}-1\right) & \text { for } \mathrm{Re}_{\mathrm{d}}<40 \\
f_{l}=0.0524 \sqrt{\frac{\operatorname{Re}_{\mathrm{d}}}{2 \mathrm{Ro}_{\mathrm{o}}}} & \text { for } 40<\mathrm{Re}_{\mathrm{d}}<100
\end{array}
$$




\subsubsection{Multiphase turbulence modeling}

Turbulence in multi-phase flow is a complex and little understood phenomenon. A dispersed phase can either dampen or enhance the turbulence, depending on the volume fraction, droplet size and density difference between the dispersed and continuous phase. Also phase inversion, which is likely in these bulk separators, further complicates the description of the flow, since then the material properties of the continuous and dispersed phase interchange. Within the framework of the two-fluid model, models exist which predict the effect of the disperse phase on the continuous phase and predict turbulent flow behavior of a dilute dispersed phase $[35,42,64,103]$. These models presume a situation in which one phase can be clearly defined as the continuous phase and its turbulence is seen as the dominant process, while the disperse phase is dilute. However, in the current application the phases separate so regions will form where this condition is no longer satisfied. Moreover, for high volume fractions the difference in the fluctuating velocity of the phases tends to zero [92].

Therefore, in this work a homogeneous turbulence model is applied, which means that both oil and water share the same turbulence field. Consequently, identical Reynolds stresses are used in the momentum equations for both phases. This type of modeling is advised for phase-separating flow as are encountered here [3]. The SSG turbulence model for single-phase flow is readily extended to two-phase flows by replacing in the models the density and the velocity by the mixture density and the mixture velocity, respectively.

\subsection{Ansys CFX}

The software package Ansys CFX 14.0 is used to solve the governing equations. To compute the solution, the computational domain is first discretized by dividing it into elements. ICEM CFD was used for the construction of these computational meshes. The meshes used for the numerical results discussed in this thesis are all block-structured hexahredral meshes. For use in Ansys CFX, these meshes are converted to unstructured meshes. However, is only means that the systematic numbering of the nodes is lost, but the elements stay in place. The hexahedral elements are used for their favorable effect on numerical quality [111], that is lower computational cost for equal accuracy compared to a mesh consisting of, for example, tetrahedral elements.

In Ansys CFX the equations are discretized using a finite-volume formulation. Control volumes are constructed around the nodes of the elements. These control volumes are employed as the volumes to which the integral conservation form of the Navier-Stokes equations is applied. The surface integrals appearing in this formulation are then approximated by the fluxes across the element boundaries [10]. Ansys CFX uses a collocated, vertex based, finite volume method. This means that both velocities and pressures are stored at the nodes of the elements. Linear shape functions are employed to find the variables at locations in between the nodes by interpolation. To prevent checkerboard instabilities of the pressure associated with this type of discretization, a pressure-velocity coupling similar to that of Majumdar [70] was used. An implicit method is used for the time discretization. The discretization of the equations leads to a scheme that has 
second-order accuracy in time and near second-order accuracy in space, while the solution stays bounded.

Ansys CFX uses a coupled solver for the velocity and pressure is which these variables are solved simultaneously. For each time step this nonlinear set of equations is linearized and it is iteratively solved using an incomplete lower upper factorization technique. To attain faster convergence an algebraic multigrid method [87] is used in combination with this factorization technique. This linearized procedure is repeated with the updated variables until user-defined convergence criteria are met.

It was found that time-accurate (transient) simulations were necessary to arrive at a converged solution. Convergence stalled when steady-state simulations were attempted. 


\section{CHAPTER 3}

\section{Design of separator}

\subsection{Introduction}

To investigate the flow and the separation characteristics in a centrifugal separator, a prototype had to be developed. This chapter discusses the design processes, comparison of different design configurations, selection of a separator prototype and the experimental setup constructed at the TUD.

One of the design specifications is that the separator does not contain any moving parts, that is the swirling motion has to be flow induced. Various configurations have been considered as prototype. They are compared in terms of pressure drop and separation efficiency.

Earlier Dirkzwager [29] designed and experimentally investigated an axial centrifugal separator. This configuration was further developed by Delfos et al. [27]. Here the term axial centrifugal separator is used for a device in which the swirling flow is generated by vanes inside the piping, that is it is generated in-line. This axially incoming feed is the differentiating feature when comparing the axial centrifugal separator with the more common tangential hydrocyclones. In the latter the incoming flow is tangentially injected in such a way that swirling flow is generated. An advantage of the axial centrifugal separator over the tangential hydrocyclone is that the former is more compact. For the present project a new in-line centrifugal separator has been designed. 


\subsection{Design of internal swirl element}

In the new design, the swirling flow is generated by a stationary internal swirl element (ISE) which is placed within a pipe with a $100 \mathrm{~mm}$ internal diameter. This ISE consists of a central body which is equipped with vanes that deflect the flow. The vanes are attached to the pipe wall in order to fix the ISE in place. The general layout of the ISE is shown in figure 3.1. Key design features are the vane geometry, central body radius $R_{i n}$, nose section and tail section.

\subsubsection{Requirements of the velocity field}

For the design of the vanes the required velocity fields at the trailing edge should be known. The first step of the design process is to estimate that data. Unfortunately, swirling flow can lead to complex flow patterns which are not known a priori and therefore an engineering approach will be taken to develop the prototype based on many assumptions. The azimuthal velocity distribution should be such that an oil droplet will migrate quickly towards the center of the pipe. The drag on the droplet is assumed to be given by Stokes' law

$$
D_{S t}=3 \pi \mu_{w}|\Delta \mathbf{u}| d
$$

In the present analysis the axial and azimuthal components of the velocity difference are negligible compared to the radial component. Also the continuous, water phase is assumed to have negligible radial velocity, therefore the velocity difference is equal to the radial velocity of the droplet $|\Delta \mathbf{u}| \approx u_{r}$. This drag force is balanced by the centrifugal body force acting on the droplet

$$
F_{c e n}=\Delta \rho \frac{\pi d^{3}}{6} \frac{u_{\theta}^{2}}{r_{d}}
$$

Here $\Delta \rho$ is the density difference between the phases and $r_{d}$ is the radial location of the droplet. The radial velocity of the droplet then follows as

$$
u_{r}=-\frac{\Delta \rho d^{2} u_{\theta}^{2}}{18 \mu_{w} r_{d}}
$$

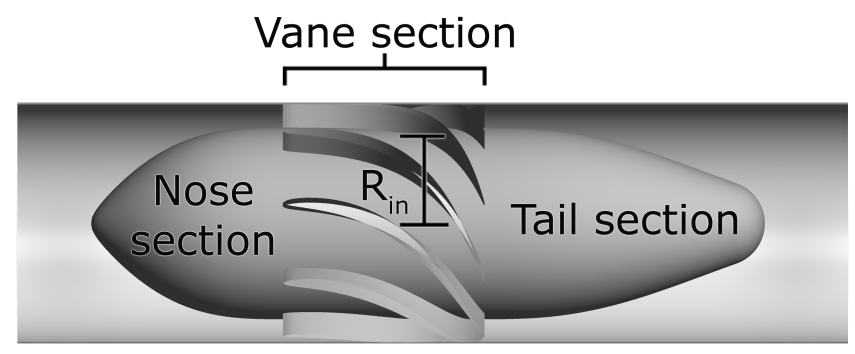

Figure 3.1: Internal swirl element with key design features indicated. 
Note that the effects of turbulence are neglected in this analysis.

Experimental data on swirling pipe flow shows that the azimuthal component of velocity has a distribution similar to that of a Rankine vortex, that is with a solid-body rotation in the center and a potential flow vortex at larger radius $[29,57,107]$. This velocity distribution is approximated by the following relation:

$$
\begin{array}{cll}
u_{\theta}(r, z)=U_{\theta}(z) \frac{r}{R_{c}} & \text { for } & 0<r<R_{c} \\
u_{\theta}(r, z)=U_{\theta}(z) & \text { for } & R_{c}<r<R
\end{array}
$$

Here $U_{\theta}(z)$ is an azimuthal velocity scale. $R_{C}$ is the radius of the core which has a solidbody rotation, the ratio $R_{C} / R \approx 0.25[29,57,107]$. This azimuthal velocity distribution is illustrated in figure 3.2. Included in figure 3.2 are the experimental data from Dirkzwager [29], which shows the similarity. It is noted in passing that the distribution in equation (3.4) might be improved by replacing the radially constant distribution in $r \in\left[R_{c}, R\right]$ by $u_{\theta}(r, z)=U_{\theta}(z) R_{c} / r$. This may give an improved fit of the data, while midly complicating the analysis. However, in the present study the distribution given in equation (3.4) has been used.

The intended use of the prototype is the separation of dense dispersions for which there will be an oil core in the center. Therefore, the oil droplet does not have to travel to the geometrical center, reaching the edge of the oil core will be adequate. For an oil volume fraction $\alpha$ of $10 \%$ the oil core will already be larger than $R_{C}$. Hence, only the outer, constant velocity part will be taken into account. An expression for the radial location of the droplet as function of the axial distance follows from

$$
u_{r}=\frac{d r_{d}}{d t}=\frac{d z_{d}}{d t} \frac{d r_{d}}{d z_{d}}=u_{z} \frac{d r_{d}}{d z_{d}}
$$

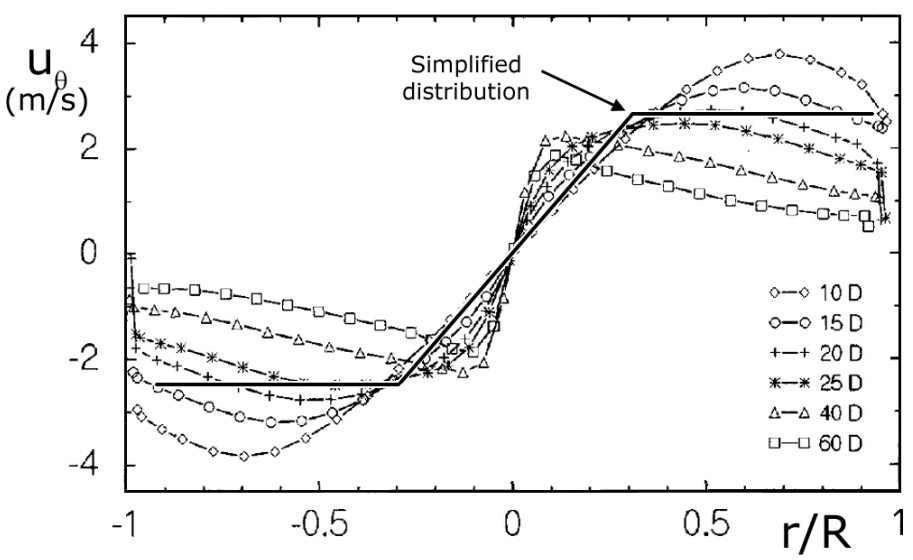

Figure 3.2: Radial distribution of azimuthal velocity in swirling pipe flow at various axial locations measured by Dirkzwager [29] and approximation of azimuthal velocity distribution (solid line, no symbols) used in design of ISE. 


\begin{tabular}{|ll||ll|}
\hline$\Delta \rho=200 \mathrm{~kg} / \mathrm{m}^{3}$ & $R$ & $=0.05 \mathrm{~m}$ \\
$d$ & $=100 \mu \mathrm{m}$ & $\alpha$ & $=0.1$ \\
$L$ & $=1 \mathrm{~m}$ & $\mu_{w}$ & $=1 \times 10^{-3}$ Pa.s \\
$u_{z, b}$ & $=2 \mathrm{~m} / \mathrm{s}$ & $C_{\text {decay }}$ & $=0.04$ \\
\hline
\end{tabular}

Table 3.1: Parameters used in estimation of required azimuthal velocity in separator.

Here the axial bulk velocity $u_{z, b}$ will be used for $u_{z}$. The dependence of $u_{\theta}$ on $z$ is represented by modeling the decay of the swirl according to

$$
U_{\theta}(z)=U_{\theta}(0) e^{-C_{\text {decay }} z / 2 R}
$$

Here $\mathrm{U}_{\theta}(0)$ is the azimuthal velocity just downstream of the ISE. Also, $C_{\text {decay }}$ is an experimentally determined parameter set to 0.04 [29]. Equations (3.5) and (3.6) are substituted into equation (3.3) and yield, after rewriting and integration, the radial position of an oil droplet as function of the downstream direction $z$ :

$$
r_{d}^{2}(z)=r_{d}^{2}(0)-\frac{2 \Delta \rho d^{2} U_{\theta}^{2}(0) R}{18 \mu_{w} u_{z, b} C_{\text {decay }}}\left[1-e^{-C_{\text {decay }} z / R}\right]
$$

In equation (3.7) $r_{d}(0)$ is the initial radial position of the droplet at the inlet, here the worst-case scenario is taken; $r_{d}(0)=R$. The separator length is given by $L$. At $z=L$ the radial position of the droplet should be

$$
r_{d}(L)=R \sqrt{\alpha}
$$

After rewriting, equation (3.7) will give the azimuthal velocity $U_{\theta}(0)$

$$
U_{\theta}(0)=\sqrt{\frac{9 \mu_{w} u_{z, b} C_{\text {decay }} R(1-\alpha)}{\Delta \rho d^{2}\left(1-e^{-C_{\text {decay }} L / R}\right)}}
$$

The parameters used in the calculation of the azimuthal velocity are listed in table 3.1. These parameters are presumed to have realistic and relevant values. This leads to a required tangential velocity $U_{\theta}(0)=5.4 \mathrm{~m} / \mathrm{s}$ to separate oil droplets of $100 \mu \mathrm{m}$ diameter in an oil-water mixture of $\alpha=0.1$. The next step is to derive the required azimuthal velocity just aft of the trailing edge of the vanes. For this purpose the conservation of axial flux of angular momentum is employed, that is:

$$
\int_{R_{i n}}^{R} u_{z, t e} 2 \pi r \rho r u_{\theta, t e} d r=\int_{0}^{R_{C}} u_{z, b} 2 \pi r \rho r U_{\theta}(0) \frac{r}{R_{C}} d r+\int_{R_{C}}^{R} u_{z, b} 2 \pi r \rho r U_{\theta}(0) d r
$$

Here the axial velocity distribution is approximated by the axial bulk velocity. $R_{\text {in }}$ is the radius of the central body at the vane section of the ISE. The vanes are mounted in the 
annular ring between the inner body and the pipe wall. $u_{z, t e}$ and $u_{\theta, t e}$ are the axial and azimuthal velocity components at the trailing edge, respectively. Dirkzwager [29] specifies his design parameters and provides measured data of velocity profiles. From this it can be derived that about $50 \%$ of the angular momentum is lost between the vane section and the first measurement station in the pipe section. Therefore, the left-hand side of equation 3.10 is multiplied with $\left(1-\epsilon_{S W}\right)$, here the loss factor $\epsilon_{S W}=0.50$. The azimuthal velocity $u_{\theta, t e}$ is assumed to be constant across the height of the narrow gap between the pipe wall and the inner body. The relation between the axial velocities is easily deduced from conservation of mass and given by

$$
u_{z, t e}=u_{z, b} \frac{R^{2}}{R^{2}-R_{i n}^{2}}
$$

From substitution of equation (3.11) in (3.10) it follows that

$$
u_{\theta, t e}=U_{\theta}(0) \frac{\left(R^{3}-\frac{1}{4} R_{C}^{3}\right)\left(R^{2}-R_{i n}^{2}\right)}{R^{2}\left(R^{3}-R_{i n}^{3}\right)\left(1-\epsilon_{S W}\right)}
$$

The use of vanes leads to a fixed ratio of azimuthal over axial velocity at the trailing edge of the vanes. To attain high azimuthal velocities a high axial velocity is therefore required. This high axial velocity is achieved by creating a narrow annular gap, in which the vanes are placed. Both the higher axial velocity, and the relatively large radius at which the vanes are placed, contribute to the increase in angular momentum. However, when the section is too narrow shear forces lead to high pressure losses and in addition may lead to droplet break-up. Therefore, a compromise has to be made. This leads to a radius of the central body of $R_{\text {in }}=40 \mathrm{~mm}$. This results in required azimuthal velocity at the trailing edge $u_{\theta, t e}=8.0 \mathrm{~m} / \mathrm{s}$ as follows from equation (3.12) using $U_{\theta}(0)=5.4 \mathrm{~m} / \mathrm{s}$ and $R_{C}=$ $12.5 \mathrm{~mm}$. Moreover, from equation (3.11) it follows that $u_{z, t e}=5.6 \mathrm{~m} / \mathrm{s}$. This yields to the required flow deflection of

$$
\arctan \left(\frac{u_{\theta, t e}}{u_{z, t e}}\right)=55^{\circ}
$$

However, in the intended application many droplets flow towards the center in the separator and they will hinder each other on their way towards to center. Moreover, the effects of turbulence, which can negatively influence the separation performance are not incorporated in this analysis. Therefore, the many approximations lead to uncertainty of the results. To counteract the uncertainty the vanes are designed with a larger deflection angle of the flow. This provides some margin. Here the required flow deflection is increased by $10^{\circ}$ from $55^{\circ}$ to $65^{\circ}$. The azimuthal velocity at the trailing edge $u_{\theta, t e}$ now increases to $11.9 \mathrm{~m} / \mathrm{s}$.

\subsubsection{Design of vane geometry}

The software program CASCADE [67] was used the find a vane shape which meets the criteria of $u_{\theta, t e}=11.9 \mathrm{~m} / \mathrm{s}$ and which has a shock-free approach of the flow at the leading 
edge of the vane. CASCADE uses a potential flow model to calculate the flow around a cascade of vanes. A cascade is a row of vanes, giving a two-dimensional approximation of the vane section of the swirl element. A sketch of the cascade with vane geometry and a graphical explanation of the main design variables is shown in figure 3.3.

The program requires the pitch, stagger angle, camber line shape, thickness distribution and fluid inlet angle as input. The pitch is the distance between vanes. The stagger angle is the angle between the chord line, that is the line from leading edge to trailing edge, and the axis of the pipe. The camber line is the curve equidistant from upper and lower surface of the vane. The camber line is given as its distance perpendicular to the chord line. As the name implies the thickness distribution gives the distance from camber line to the surface. The fluid inlet angle is set to $0^{\circ}$, that is parallel with to axis of the pipe. The program calculates the shock-free stagger angle and the fluid deflection.

A vane geometry is found which satisfies the design criteria. The vanes are based on the NACA four-digit airfoil series. The chord length $c$ is chosen equal to $100 \mathrm{~mm}$. A larger flow deflection can be accomplished with longer vanes. Flow separation will occur if the vanes are too short. Also the mechanical strength will increase for longer vanes and the connection of the vanes to the pipe wall can be made structurally more robust. However, longer vanes will create longer shear layers along their surfaces. These regions generate more turbulence which will lead to more losses and, quite importantly, more droplet breakup. The current length is presumed to be a compromise between the negative effect of length on shear and the positive effect on mechanical strength.

The camber line has a parabolic shape with its maximum deflection located at $50 \%$ of the chord length. The maximum distance $\Delta m$ from the chord line is given as fraction of

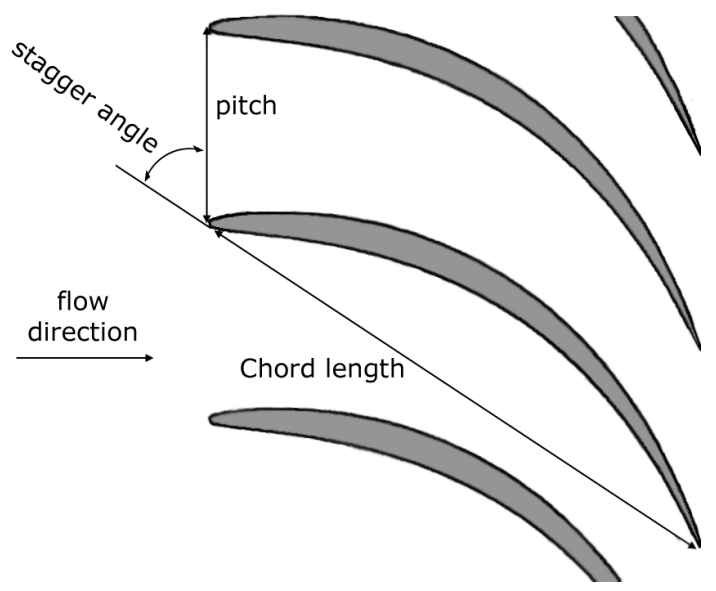

Figure 3.3: Sketch of the cascade showing vane geometry and explanation of the main design variables. 


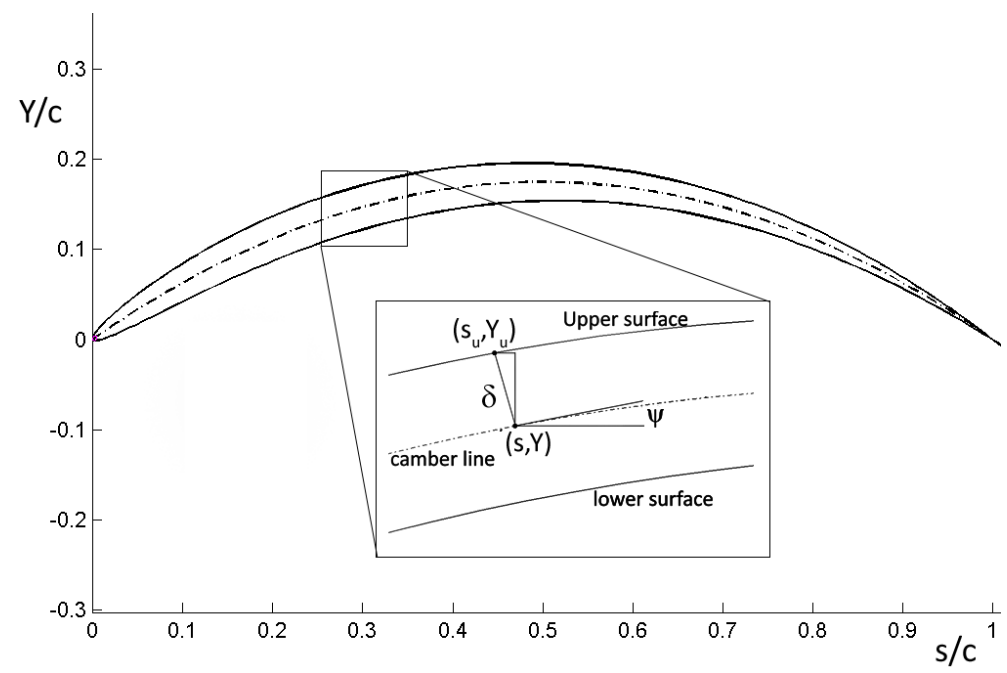

Figure 3.4: Geometry of vane (solid) in local coordinate system showing the camber line (dotted). Detailed view shows visual explanation of equation (3.16).

the chord length: $\Delta m=0.1751$. The camber line shape is given by

$$
Y(s)=\frac{4 \Delta m}{c}\left(c s-s^{2}\right)
$$

Here $s$ is the coordinate along the chord line starting with $s=0$ at the leading edge and ending with $s=c$ at the trailing edge. The thickness distribution $\delta(s)$ is given by the one for the NACA four digit section

$$
\delta(s)=\frac{\delta_{\max }}{0.20} c\left(a_{0} \sqrt{\frac{s}{c}}+a_{1} \frac{s}{c}++a_{2}\left(\frac{s}{c}\right)^{2}+a_{3}\left(\frac{s}{c}\right)^{3}+a_{4}\left(\frac{s}{c}\right)^{4}\right)
$$

Here the maximum thickness $\delta_{\max }$ is scaled with the chord length $c$ and is $4.8 \%$. The coefficients are given by $a_{0}=0.2969, a_{1}=-0.1260, a_{2}=-0.3516, a_{3}=0.2843$ and $a_{4}=-0.1015$ [1]. The upper and lower surface are now formed by

$$
\begin{aligned}
s_{l}(s) & =s+\delta \sin \psi(s), \\
Y_{l}(s) & =Y(s)-\delta \cos \psi(s), \\
s_{u}(s) & =s-\delta \sin \psi(s), \\
Y_{u}(s) & =Y(s)+\delta \cos \psi(s),
\end{aligned}
$$

Here $\psi$ is the slope of the camber line shape with respect to the chord line.

$$
\psi=\arctan \left(\frac{d Y}{d s}\right)=\arctan \left(\frac{4 \Delta m}{c}(c-2 s)\right)
$$




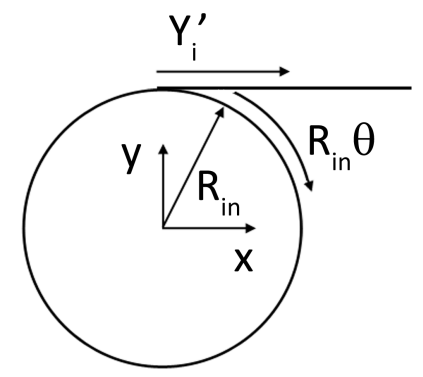

Figure 3.5: Sketch of wrapping 2D vane geometry around central body. Parameters in coordinate transformation are shown.

The resulting vane geometry given by equation (3.16) and the camber line given by equation (3.14) are shown in figure 3.4. A close-up in the figure shows a visual explanation of equation (3.16). A shock-free approach of the fluid is achieved when the stagger angle is set to $-33.1^{\circ}$. The vane geometry is thus rotated over an angle of $-33.1^{\circ}$; this is shown in figure 3.3. The transformation is given by

$$
\begin{aligned}
Y_{i}^{\prime} & =\sqrt{s_{i}^{2}+Y_{i}^{2}} \sin \left(\arctan \left(\frac{Y_{i}}{s_{i}}\right)-\frac{33.1}{180} \pi\right) \\
s_{i}^{\prime} & =\sqrt{s_{i}^{2}+Y_{i}^{2}} \cos \left(\arctan \left(\frac{Y_{i}}{s_{i}}\right)-\frac{33.1}{180} \pi\right)
\end{aligned}
$$

Here the subscript $i$ is $u$ or $l$, giving the upper and lower surface in the rotated coordinates, respectively. The angle of the camber line at the trailing edge of the vane with the axial direction is now $68.1^{\circ}$. The angle of the suction and pressure side is $71.1^{\circ}$ and $65.9^{\circ}$, respectively. Thus, the angle of the final vane design is slightly higher than the design angle of $65^{\circ}$. The axial length of the vane is $83.5 \mathrm{~mm}$. In the present internal swirl element, 9 vanes are used, this gives an average pitch of $31.4 \mathrm{~mm}$. The use of more vanes will make it easier to obtain a larger flow deflection and to prevent flow separation. However, too many vanes will lead to additional frictional losses and a larger pressure drop due to a larger blockage of the flow area. The lift per vane is known to decrease if vanes are added. A lower lift will generate less fluid deflection. Increasing the number of vanes will eventually lead to a decrease in fluid deflection. To check whether this situation is already reached for 9 vanes a further analysis with CASCADE has been carried out. This showed that this is not the case since more lift is obtained when one or two vanes are added. However, adding these vanes would lead to the negative effects discussed above. The two-dimensional geometry is wrapped around the central body. The contour of a vanes on the central body in the global frame of reference is given by

$$
\begin{aligned}
& x_{i n}=R_{i n} \cos \left(Y_{i}^{\prime} / R_{i n}\right), \\
& y_{i n}=R_{i n} \sin \left(Y_{i}^{\prime} / R_{i n}\right), \\
& z_{i n}=s_{i}^{\prime}
\end{aligned}
$$




\begin{tabular}{|ll||ll|}
\hline$\Delta x_{n}=80 \mathrm{~mm}$ & $f_{n}(0)=0$ \\
$b_{1}$ & $=0.9$ & $f_{n}^{\prime}(0)=3-15 / 8 b_{1}-3 / 8 b_{2}$ \\
$b_{2}$ & $=6.0$ & $f_{n}(1)=1-b_{1}-b_{2}$ \\
$R_{\text {in }}=40 \mathrm{~mm}$ & $f_{n}^{\prime}(1)=-1 / 2 b_{1}-3 / 2 b_{2}$ \\
\hline
\end{tabular}

Table 3.2: Parameters used in design of nose section.

Figure 3.5 depicts this coordinate transformation. It shows the cross-flow plane of the vane section with the central body with radius $R_{i n}$ with the planar vane geometry at the top. The azimuthal coordinate of a point on the planar vane is found by using $\theta=Y_{i}^{\prime} / R_{i n}$. Finally the geometry is radially extruded to the pipe wall to form a three-dimensional vane geometry.

\subsubsection{Design of nose section}

Now that the central body with vane section has been defined the next step is to design the nose section. The nose section will have a hemispherical-like shape and should smoothly accelerate the flow towards the vane section and it should not have any abrupt changes in shape, which may introduce disturbances to the flow. This can be accomplished by ensuring that the radius, the slope and its derivative of the nose section match the ones of the vane section at the interface between these two sections. The approach taken here is to define the shape of the nose by revolving a polynomial around the pipe axis. The shape of nose cap is given by the following 6-parameter expression:

$$
\begin{aligned}
r\left(\xi_{n}\right)=R_{i n} & {\left[b_{1} \xi_{n}^{1 / 2}+b_{2} \xi_{n}^{3 / 2}+f_{n}(0) P_{1}\left(\xi_{n}\right)\right.} \\
& \left.+f_{n}^{\prime}(0) P_{3}\left(\xi_{n}\right)+f_{n}(1) P_{2}\left(\xi_{n}\right)+f_{n}^{\prime}(1) P_{4}\left(\xi_{n}\right)\right]
\end{aligned}
$$

The dimensionless coordinate $\xi_{n}$ is defined as

$$
\xi_{n}=\frac{x_{n}-x_{n o s e}}{\Delta x_{n}}
$$

Here $\Delta x_{n}=x_{\text {vanes }}-x_{n o s e}=80 \mathrm{~mm}$. The axial coordinates $x_{n o s e}$ and $x_{\text {vanes }}$ are the begin point of the nose and vane section, respectively. Furthermore, $P_{1}$ to $P_{4}$ are Hermite polynomials which are multiplied by weight factors $f_{n}$ and $f_{n}^{\prime}$. These factors $f_{n}$ and $f_{n}^{\prime}$ are included because they can be chosen such that the function describing the nose cap meets the following matching criteria.

$$
\begin{aligned}
\left.r\right|_{\xi_{n}=0} & =0 \\
\left.r\right|_{\xi_{n}=1} & =R_{i n}, \\
\left.\frac{d r}{d x_{n}}\right|_{\xi_{n}=1} & =0 \\
\left.\frac{d^{2} r}{d x_{n}^{2}}\right|_{\xi_{n}=1} & =0
\end{aligned}
$$




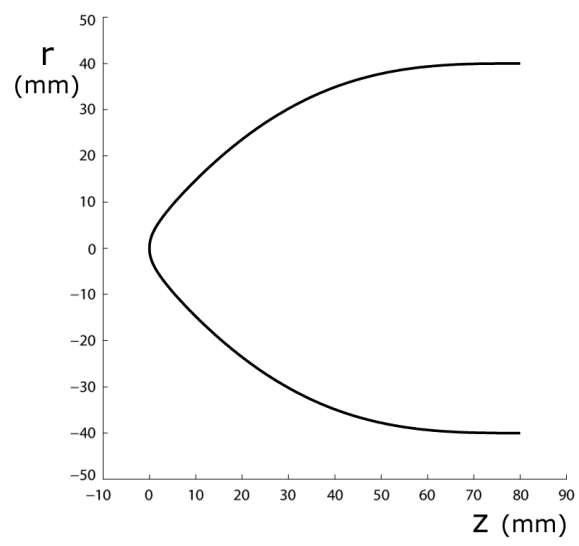

Figure 3.6: Shape of nose section as given by equation 3.20 with parameters from table 3.2.

These four constraints leave two parameters, $b_{1}$ and $b_{2}$, to be chosen. The $\xi_{n}^{1 / 2}$ and $\xi_{n}^{3 / 2}$ terms are included to achieve a suitable, hemispherical-like nose shape. This description has been obtained by trial-and-error. The parameters used in the design of the nose cap are listed in table 3.2. The weights $b_{1}$ and $b_{2}$ can be chosen freely and are determined heuristically. Figure 3.6 shows the shape of the nose section obtained with this procedure. Further information on Hermite polynomials and the derivation of the parameters can be found in appendix $A$.

\subsubsection{Design of tail section}

For the tail section a similar procedure is followed as for the nose section. This time a quintic polynomial is employed so that it is possible to determine the radius, slope and second derivative at the begin and endpoint of the tail section. The 6-parameter expression for the tail is

$$
\begin{aligned}
r\left(\xi_{t}\right)= & R_{\text {in }}\left[f_{t}(0) Q_{1}\left(\xi_{t}\right)+f_{t}^{\prime}(0) Q_{3}\left(\xi_{t}\right)+f_{t}^{\prime \prime}(0) Q_{5}\left(\xi_{t}\right)\right. \\
& \left.+f_{t}(1) Q_{2}\left(\xi_{t}\right)+f_{t}^{\prime}(1) Q_{4}\left(\xi_{t}\right)+f_{t}^{\prime \prime}(1) Q_{6}\left(\xi_{t}\right)\right]
\end{aligned}
$$

Here $Q_{1}$ to $Q_{6}$ are the quintic Hermite polynomials given in the appendix B. The variable $\xi_{t}$ is the dimensionless axial coordinate defined as

$$
\xi_{t}=\frac{x_{t}-x_{t a i l}}{\Delta x_{t}}
$$


Here $\Delta x_{t}=x_{t i p}-x_{t a i l}=200 \mathrm{~mm}$. At the transition from vane section to tail section the radius, slope and gradient of the slope should match, that is

$$
\begin{aligned}
\left.r\right|_{\xi_{t}=0} & =R_{i n} \\
\left.\frac{d r}{d x_{t}}\right|_{\xi_{t}=0} & =0 \\
\left.\frac{d^{2} r}{d x_{t}^{2}}\right|_{\xi_{t}=0} & =0
\end{aligned}
$$

At the tip, the boundary conditions are

$$
\begin{aligned}
\left.r\right|_{\xi_{t}=1} & =0 \\
\left.\frac{d r}{d x_{t}}\right|_{\xi_{t}=1} & =-\tan \phi_{t}, \\
\left.\frac{d^{2} r}{d x_{t}^{2}}\right|_{\xi_{t}=1} & =0
\end{aligned}
$$

Here $\phi_{t}$ is the angle of the tip of the tail section with respect to the pipe axis. After a trail-and-error process, it has been set to $10^{\circ}$. As detailed in appendix $B$, the only nonzero parameters are $f_{t}(0)=1$ and $f_{t}^{\prime}(1)=-\Delta x_{t} / R_{\text {in }} \tan \phi_{t}$. When these requirements are substituted in equation (3.23) the expression becomes

$$
r\left(\xi_{t}\right)=R_{i n} Q_{1}\left(\xi_{t}\right)-\Delta x_{t} \tan \phi_{t} Q_{4}\left(\xi_{t}\right)
$$

For manufacturing reasons the sharp tip is replaced by a spherical cap. However, the mathematical expression (3.27) is still used for the shape upstream of this cap. The cap has a radius of $17.87 \mathrm{~mm}$ and has its center $104.12 \mathrm{~mm}$ downstream of the start of the

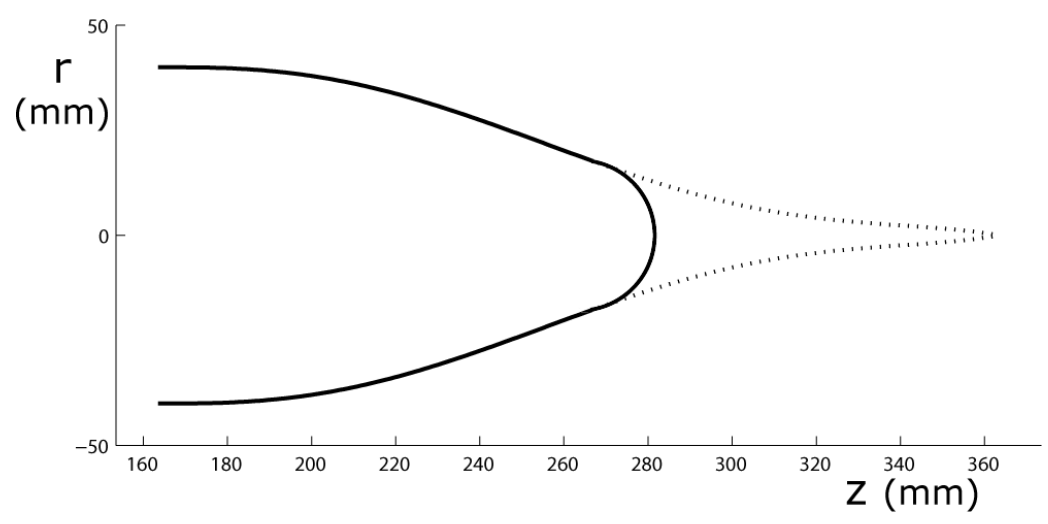

Figure 3.7: Shape of tail section. The shape of the upstream part is given by equation (3.27) with spherical cap at the end. Removed section is shown as dashed line. 

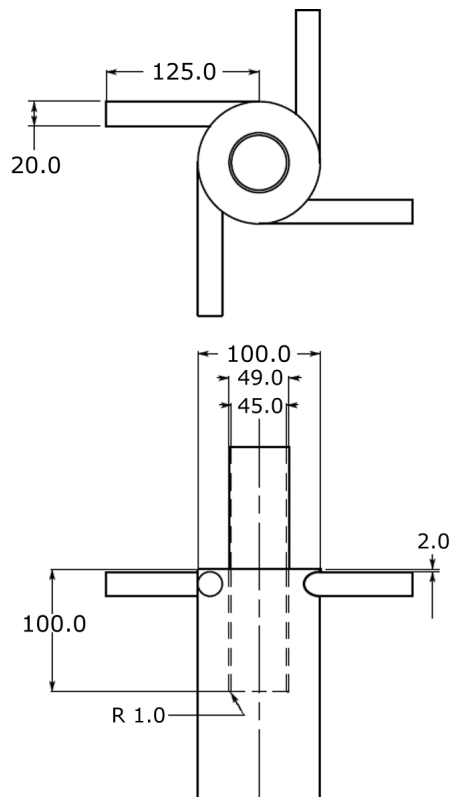

Figure 3.8: Design of hydrocyclone for comparison studies, dimensions are in $\mathrm{mm}$.

tail section $x_{t a i l}$. This coincides with $\xi_{t}=0.5206$. The dimensions of the cap are based on visual inspection. At the transition from the shape given by equation (3.27) and the spherical cap the slope and its derivative are no longer continuous. However, a smooth transition is beneficial in the high velocity region just after the vanes. Further downstream a reversed flow region is present around the tail section. The effects on the flow of the transition between tail and cap are assumed to be negligible. The final shape of the tail is given in figure 3.7. The combination of the individual sections leads to the design of the ISE given in figure 3.1.

\subsection{Comparison with alternative separator geometries}

The performance of the ISE is compared to that of a simple hydrocyclone. This preliminary study should bring to light what potential advantages and drawbacks the various designs have and this comparison will lead to a selection of a prototype for further studies.

\subsubsection{Alternative configuration}

The geometry of the hydrocyclone is given in figure 3.8. The hydrocyclone has four tangential inlets, each a cylinder with a $20 \mathrm{~mm}$ diameter. The light-phase outlet (LPO) at the top of the separator has a diameter of $45 \mathrm{~mm}$. This vortex finder penetrates 100 
$\mathrm{mm}$ into the pipe. The inlet velocity in each of the four inlets is $12.5 \mathrm{~m} / \mathrm{s}$, leading to a flow rate of $56.5 \mathrm{~m}^{3} / \mathrm{hr}$, that is the same flow rate as for the ISE configuration. The sizing of the four inlets is such that just downstream of the inlet section in the hydrocyclone, the axial flux of angular momentum is very similar to that in the ISE just downstream of the vanes. This allows for a meaningful comparison.

Extraction of the oil can be done in two ways; co-current or counter-current. In co-current separation the separated oil and water both flow downstream where they are subsequently extracted separately. In a counter-current separator the separated oil and water streams have opposite directions. A counter-current in-line separator with similar design as the ISE is already available on the market [98] and will not be investigated in the present thesis. Therefore, the ISE will be equipped with a pick-up tube placed concentrically at the downstream end of the separator. For completeness, both co-current and counter-current applications of the hydrocyclone are investigated here. A co-current hydrocyclone can simply be obtained by blocking the vortex finder. This configuration will be designated by HC-BVF. Likewise the counter-current configuration, which has an open vortex finder, is abbreviated by HC-OVF.

In all cases, the pipe extends $2.0 \mathrm{~m}$ downstream, measured from the tip of the tail of the ISE and the edge of the vortex finder in the hydrocyclone. This straight pipe is followed by a short tapered section. The tapered section is $0.2 \mathrm{~m}$ long and reduces the pipe diameter from 100 to $45 \mathrm{~mm}$. In the tapered section the flow is accelerated and therefore it will prevent reversed flow as, for instance, is often seen in a counter-current hydrocyclone. Later designs will feature a more refined extraction geometry. However, for the present purposes a tapered section is adequate. In this tapered section a conical pick-up tube is placed. This outlet is defined as the heavy-phase outlet (HPO) The resulting geometries are presented in figure 3.9.

The calculations have been carried out using Ansys CFX 14.0 using a mesh of $0.58 \mathrm{M}$ and $0.52 \mathrm{M}$ hexahedral elements for the ISE and the hydrocyclone, respectively. As this has been a exploratory study to select a prototype for further studies, the meshes are rather coarse.

\subsubsection{Comparison results}

To ensure a fair comparison, similar levels of swirl intensity should be achieved in the different configurations. The swirl number $S$ can be interpreted as a measure of the

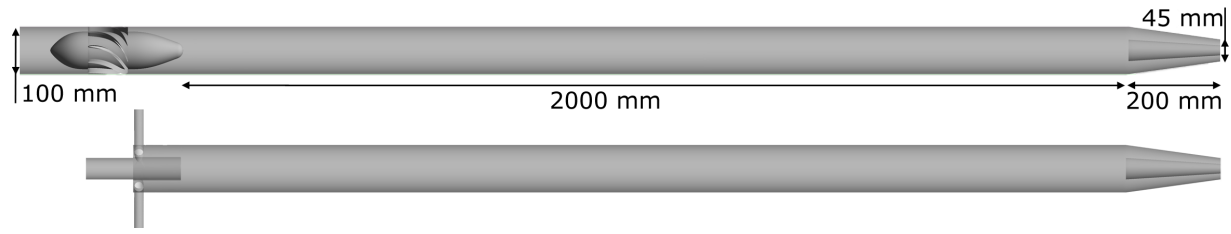

Figure 3.9: ISE (top) and HC-BVF (bottom) geometries used in preliminary study for prototype selection. In counter-current HC-OVF geometry the downstream pick-up tube is removed. 


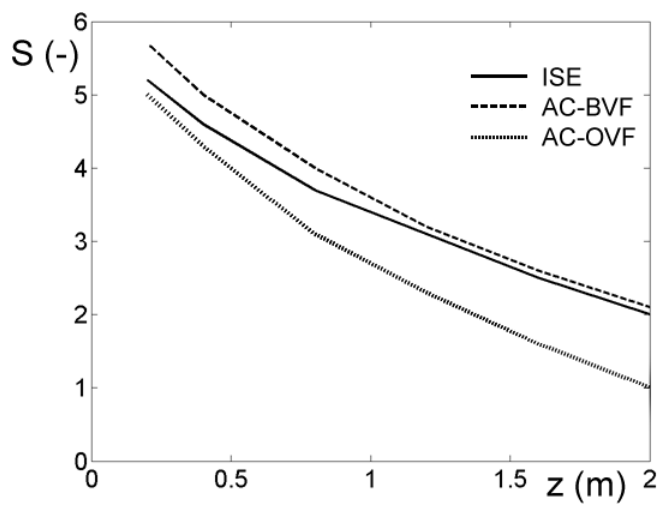

Figure 3.10: Comparison of evolution of $\mathrm{S}(z)$ for design alternatives.

intensity of the swirl. For single-phase water flow, it is defined as [57]

$$
\mathrm{S}(z)=\frac{\int_{0}^{R} \int_{0}^{2 \pi} \rho_{w} u_{\theta} r u_{z} r d \theta d r}{\rho_{w} \pi R^{3} u_{z, b}^{2}}
$$

The swirl number gives a measure of the ratio of the flux of azimuthal and axial momentum. The azimuthal velocity can be related to the g-forces the dispersed phase will be subjected to, while the bulk velocity can be related to the residence time. The evolution of $\mathrm{S}$ with $z$ for the three configurations is given in figure 3.10, showing the decay of swirl as function of $z$. As can be seen the two co-current designs show a very similar evolution of the swirl number S, although the ISE is starting with a slightly lower swirl intensity. Therefore, differences in performance can be attributed to the design and not to the inlet conditions. The counter-current version AC-OVF shows a lower swirl number than the ISE and the AC-BFV configurations at every axial location, which should be taken into account when evaluating the designs. The key parameters for comparison are the pressure drop over the separator and the separation efficiency.

\section{Pressure drop}

The swirl is generated at the expense of static pressure. The amount of pressure drop has economic relevance since it often has to be restored in order to transport the produced fluids. Here both the static and total pressure are considered. The total pressure should be considered as an unbiased comparison, since the inlet and outlet velocities differ for the different configurations. In addition, the total pressure can be used to estimate how much static pressure can be recovered. Moreover, the decrease in total pressure represents the losses in the separator. The tapering at the end of the pipe leads to high velocities and therefore the pressure drops sharply near the end of the separator. However, the exit geometry is identical for all cases and therefore a fair comparison between the configurations can be made. 


\begin{tabular}{|l||c|c|c|}
\hline & ISE & HC-BVF & HC-OVF \\
\hline \hline heavy-phase outlet - inlet & & & \\
$\Delta \bar{p}$ (bar) & 2.3 & 1.8 & 2.0 \\
$\Delta \bar{p}_{\text {tot }}$ (bar) & 1.3 & 1.6 & 1.8 \\
\hline \hline light-phase outlet - inlet & & & \\
$\Delta \bar{p}$ (bar) & 4.2 & 3.5 & 1.8 \\
$\Delta \bar{p}_{\text {tot }}$ (bar) & 2.0 & 2.2 & 2.4 \\
\hline
\end{tabular}

Table 3.3: Area-averaged static and total pressure difference $\Delta \bar{p}$ and $\Delta \bar{p}_{t o t}$ between inlet and outlet.

Table 3.3 gives the drop in static and total pressure between the inlet and outlets for the three configurations. The pressure drop observed in the ISE is the largest, however, this is due to the low inlet velocity which leads to a large pressure drop necessary to generate the swirl. Examination of the drop in total pressure shows that the ISE has actually the lowest losses. The highest losses can be seen for the HC-OVF. The swirl numbers indicate that the ISE has a lower swirl intensity. To operate the other separators at the same swirl intensity, the other co-current separator's inlet velocity needs to be reduced. This will lead to smaller total pressure losses, reducing the difference between the co-current separators somewhat.

In general, the numerical results show a large drop in pressure from the inlet to a location just after the ISE or vortex-finder section of the HC-BVF and HC-OVF. In contrast, further downstream the pressure, averaged over the cross-section, decreases only slightly.

\section{Separation characteristics}

Of large importance is the separation efficiency of the separator. The separation capability has been assessed using the two-fluid framework discussed in section 2.4. The inlet oil volume fraction is set to 0.25 , representing a feed from a high watercut oil field. The mass flow split through the oil outlet is set to 0.3 , that is

$$
\frac{\dot{m}_{L P O}}{\dot{m}_{\text {inlet }}}=0.3
$$

The mass fraction of oil at the inlet is lower than 0.3 , therefore this mass flow setting will likely result in cleaner water at the water outlet rather than purer oil at the oil outlet. Clean water is often preferred over dry oil due to increasingly stringent regulations on water discharge [36]. An oil droplet size needs to be specified for the Ishii-Zuber drag law. Here a droplet diameter of $100 \mu \mathrm{m}$ is chosen.

To measure the separation efficiency the following expression is often used $[40,50]$

$$
\eta_{1}=\frac{Q_{o, L P O}}{Q_{o, \text { inlet }}}
$$




\begin{tabular}{|c||c|c|c|c|c|c|c|}
\hline & $\alpha_{H P O}$ & $\alpha_{L P O}$ & hold up & $\eta_{1}$ & $\frac{\Delta Q_{o}}{Q_{o, \text { inlet }}}$ & $\eta_{2}$ & $\frac{1}{1-F S} \frac{\Delta Q_{o}}{Q_{o, \text { inlet }}}$ \\
$(\%)$ & $(\%)$ & $(\%)$ & $(\%)$ & $(\%)$ & $(\%)$ \\
\hline \hline ISE & 1.4 & 72 & 39 & 96 & 0.21 & 96 & 0.32 \\
HC-BVF & 3.7 & 70 & 41 & 90 & 0.06 & 90 & 0.08 \\
HC-OVF & 0.6 & 72 & 28 & 98 & 0.46 & 98 & 0.70 \\
\hline
\end{tabular}

Table 3.4: Separation characteristics for the different configurations.

Here $Q_{o, L P O}$ and $Q_{o, \text { inlet }}$ are oil flow rates at the LPO and inlet, respectively. The separation efficiency $\eta_{1}$ thus gives the fraction of the incoming oil that is separated. For convenience, this is rewritten in terms of $Q_{o, H P O}$, which is the oil flow rate at the HPO, using $Q_{o, \text { inlet }}=Q_{o, H P O}+Q_{o, L P O}$

$$
\eta_{1}=1-\frac{Q_{o, H P O}}{Q_{o, \text { inlet }}}
$$

Equation 3.31 will be used to calculate $\eta_{1}$. However, this measure does not incorporate the flow split FS. The flow split is defined as the volumetric flow rate through the LPO divided by the total flow rate.

$$
F S=\frac{Q_{L P O}}{Q_{\text {inlet }}}
$$

As a result the efficiency $\eta_{1}$ will approach unity for a flow split approach unity. In these cases the outgoing mixture from the LPO has a similar oil volume fraction as the incoming feed and hardly any separation has taken place. Therefore, an additional efficiency measure is introduced [117]

$$
\eta_{2}=1-\frac{\alpha_{H P O}}{\alpha_{\text {inlet }}}=1-\frac{1}{1-F S} \frac{Q_{o, H P O}}{Q_{o, \text { inlet }}}
$$

When rewritten in terms of oil volume fluxes, it is clear that the flow split is incorporated into this measure. Since efficiency definition (3.31) as well as definition (3.33) are often used in the literature, both measures will be given for two-phase flow results.

A small oil imbalance is present in the results, that is, the flow pattern has not yet fully established itself.

$$
\Delta Q_{o}=Q_{o, \text { inlet }}-Q_{o, H P O}-Q_{o, L P O}
$$

This imbalance could reduced further by continuing the computation for a longer time. However, this substantially increases the computational costs without adding much accuracy. The imbalance results in a slight error in the calculated efficiencies since the current $Q_{o, H P O}$ can deviate from the $Q_{o, H P O}$ in the operational state. Therefore, the lower and 
the upper limit for the efficiencies $\eta_{1}$ and $\eta_{2}$ are given by

$$
\begin{aligned}
\text { if } \Delta Q_{o}>0 \quad & \\
\eta_{1}^{-} & =1-\frac{Q_{o, H P O}+\Delta Q_{o}}{Q_{o, \text { inlet }}} \\
\eta_{1}^{+} & =1-\frac{Q_{o, H P O}}{Q_{o, \text { inlet }}} \\
\eta_{2}^{-} & =1-\frac{1}{1-F S} \frac{Q_{o, H P O+\Delta Q_{o}}}{Q_{o, \text { inlet }}} \\
\eta_{2}^{+} & =1-\frac{1}{1-F S} \frac{Q_{o, H P O}}{Q_{o, \text { inlet }}} \\
\text { if } \Delta Q_{o}<0 \quad & =1-\frac{Q_{o, H P O}}{Q_{o, \text { inlet }}} \\
\eta_{1}^{-} & =1-\frac{Q_{o, H P O}-\Delta Q_{o}}{Q_{o, \text { inlet }}} \\
\eta_{1}^{+} & =1-\frac{1}{1-F S} \frac{Q_{o, H P O}}{Q_{o, \text { inlet }}} \\
\eta_{2}^{-} & =1 \\
\eta_{2}^{+} & =1-\frac{Q_{o, H P O-\Delta Q_{o}}}{Q_{o, \text { inlet }}}
\end{aligned}
$$

The results are summarized in table 3.4. The oil hold up is defined as the fraction of the volume in the separator occupied by the oil. Since $\Delta Q_{o}>0$, equations (3.31) and (3.33) give the upper limit of the efficiencies. $\Delta Q_{o} / Q_{o}$, inlet provides the error margin. The HC-OVF shows slightly better values, but the results for the ISE are close. The HC-BVF shows a somewhat lower efficiency and also a higher hold up of oil in the separator.

\subsubsection{Conclusion}

Changing the configuration by using tangential inlets instead of an in-line central body with vanes or by changing the method of extraction from co-current to counter-current does not result in large differences in either separation performance nor pressure drop. Since the geometries explored here are not optimized and it is expected that the modeling errors in the numerical flow simulations are appreciable, the differences seen between the configurations should not be overemphasized. Within the presumed error margins of these exploratory calculations, it appears that none of the configurations that has a clear edge over the competition. Besides having the lowest total pressure loss and a good separation efficiency, the ISE is the configuration that is most in-line. This facilitates implementation in existing pipe lines and allows for a more compact design. Such a compact design is a highly desired feature of the prototype. Therefore, the novel ISE is selected for further investigation.

The final design uses a straight outer pipe and a straight pick-up tube, as can be seen 


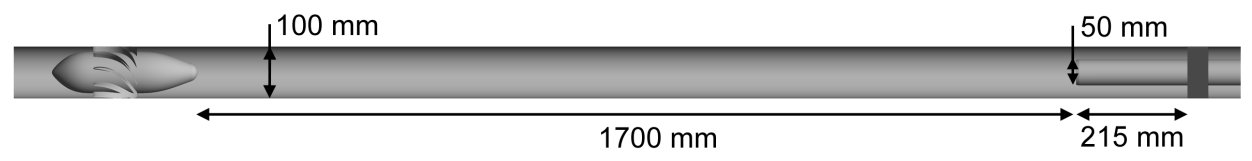

Figure 3.11: Final design of in-line separator showing ISE, pick-up tube and flow straightener.

in figure 3.11. The distance between the end of the ISE and the opening of the pickup tube is increased to $1.7 \mathrm{~m}$ based on preliminary experimental results. In the annular pipe leading towards the HPO, a flow straightener is placed $215 \mathrm{~mm}$ downstream of the entrance of the pick-up tube. This flow straightener eliminates the swirl in the HPO. In the figure it is indicated by the dark grey ring.

\subsection{Experimental setup}

At Delft University of Technology an experimental setup of the separator has been built to perform both single and two-phase flow measurements. The main components can be seen in figure 3.12 .

Two large storage vessels are placed at the ground level, one vessel contains $9 \mathrm{~m}^{3}$ water with $9 \mathrm{wt} \% \mathrm{NaCl}$ and the second vessel contains $4.2 \mathrm{~m}^{3}$ oil. The vessels feed centrifugal pumps which can produce a mixture with a widely varying oil volume fraction. These centrifugal pumps have independent frequency drives. The minimum flow rate through

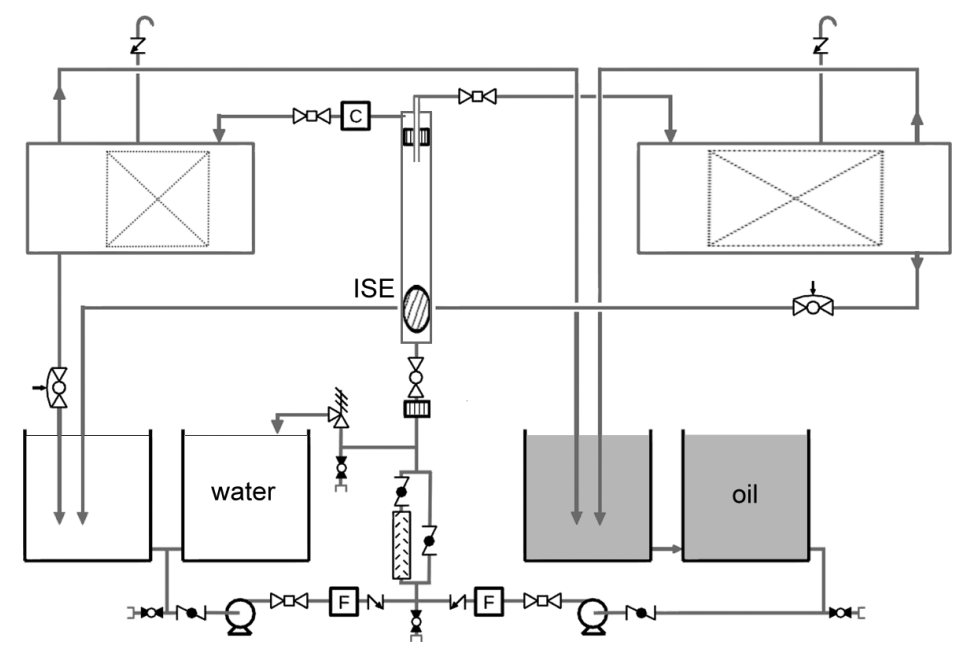

Figure 3.12: Sketch of experimental setup at the TUD showing ISE, pick-up tube, settling tanks, storage vessels and pumps. Courtesy of Laurens van Campen. 


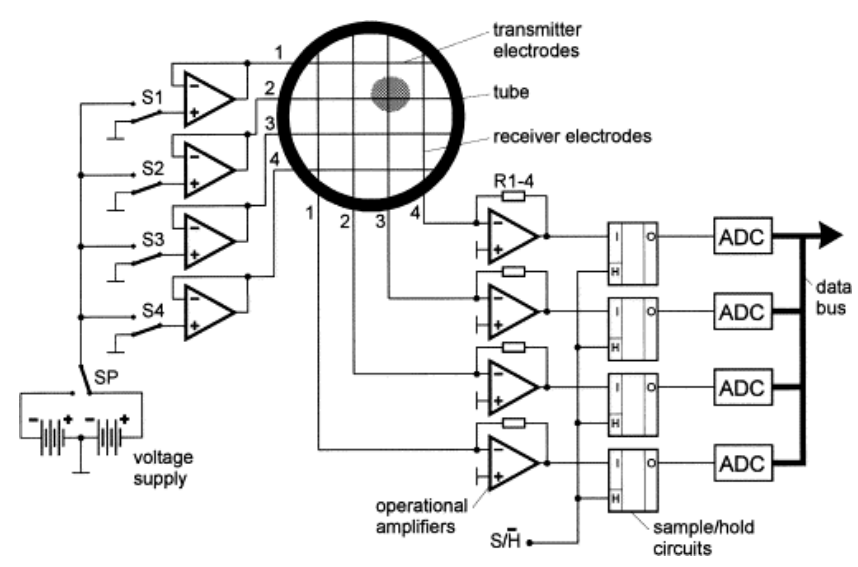

Figure 3.13: Scheme of wiremesh setup, adapted form [83].

each of the pumps is $10 \mathrm{~m}^{3} / \mathrm{hr}$, therefore a flow rate of 20 to $60 \mathrm{~m}^{3} / \mathrm{hr}$ can be reached. The typical flow rate is $56.5 \mathrm{~m} 3 / \mathrm{hr}$, leading to an axial bulk velocity of $2 \mathrm{~m} / \mathrm{s}$ for a pipe with a $100 \mathrm{~mm}$ inner diameter. Downstream of both pumps float flow meters are installed to measure the flow rate of each phase. The phases mix in the T-section, however, mixing can be enhanced by passing the oil-water mixture through a static mixer. Subsequently, the mixture enters the ISE.

Downstream of the ISE, different measurement sections can be placed. For instance, a polymethyl methacrylate (PMMA) measurement pipe segment, which provides optical access for the Laser Doppler Anemometry (LDA) measurements. Alternatively, stronger polyvinylchloride (PVC) pipe sections or sections that contain the entrances for measurement probes can be placed. The total pipe length can be varied as well. Further downstream a stainless steel pick-up tube is placed to collect the bulk of the oil. The cylindrical pickup tube has an outer diameter of $50 \mathrm{~mm}$ and wall thickness of $2 \mathrm{~mm}$. Subsequently the stream flows into the oil settling tank. The flow straightener in the annular section between the pipe wall and the pick-up tube consists of a perforated disc of PVC with holes of $5 \mathrm{~mm}$ diameter. Practice has learned that if the swirl of the flow is not eliminated, air will be sucked into the separator from the water settling tank. A coriolis flow meter, which measures mass flow rate and density, is placed in the pipe leading to the water settling tank. In combination with the float flow meters, the flow split and oil volume fractions of the two outlet flows can be determined. In the settling tanks coalescing plate packs are installed to achieve complete separation of the oil-water mixture. Finally, the oil and water flow to their respective storage vessels.

\subsubsection{Measurement techniques}

The single-phase fluid flow is examined using Laser Doppler Anemometry (LDA). In order to reduce refraction of laser light the PMMA measurement tube is surrounded by a 
square box filled with water. Since the optical transition between the PMMA tube wall and the water causes refraction, measurements are only done along lines passing through the center of the tube. An Argon laser is used, from which the $488.0 \mathrm{~nm}$ light beams are used for the measurement of the axial velocity component and the $514.5 \mathrm{~nm}$ light beams for the azimuthal velocity component. The burst correlation is conducted in a Dantec F60 BSA signal processor. The average velocity is calculated using a software package developed by Belt [8], which corrects the LDA time-averaged results for white noise, multiple validation and a bias towards high velocities.

The temperature of the mixture is monitored during operation. It rises due to the power of the pumps which is converted into heat. The increasing temperature did not show to have a significant impact on the measurements of the velocity.

For higher volume fraction of the dispersed phase, the many interfaces refract the light severely and optical measurement techniques can no longer be used. However, the difference in electrical conductance of the water and the oil can be used to visualize the flow. This can be done by the use of an electrical conductance wire-mesh system, as described by Prasser et al. [8]. Figure 3.13 depicts such an electronic circuit. However, in the current application the high salinity and high velocities require adaption to the standard wire mesh senors.

Moreover, fluid samples can be taken from various locations in the separator. These sample are analyzed in a Beckman Coulter LS 230 laser diffractiometer to determine the droplet size distribution. After extraction the sample is stabilized by a BRIJ-35 solution to prevent coalescence. Alternatively, an endoscope can be position in the flow to make in-situ recordings of the flow. Post-processing needs to be carried out in order the obtain de droplet size distribution from these images. 
CHAPTER 4

Results for single-phase flow

\subsection{Introduction}

In this chapter the results for single-phase flow are discussed. Although the separator will be used for multi-phase flow, it is useful to first analyze single-phase flow. Single-phase flow is computationally less expensive. Therefore, it has been used for the design phase in chapter 3 and for mesh convergence studies in the present chapter. Single-phase flow is also less complex than multiphase flow, making it easier to study the flow behavior in parameter studies. Moreover, single-phase flow is better understood than multiphase flow, leading to less uncertainty in the modeling and to more reliable results. Since measurement of the velocity distribution is only possible for single-phase water flows, the simulations of single-phase flow can be compared to the experimental data obtained in the experimental setup at the TUD.

The chapter is organized in the following way: first the preprocessing for the numerical simulations is discussed. Subsequently, the computed flow patterns are discussed and compared to experimental findings. Also, some interesting flow phenomena are studied in more detail. In this chapter the emphasis is more on the flow phenomena seen in the separator, while chapter 5 on two-phase flow will focus more on the separation characteristics. Finally, observed trends in flow parameters studies are reviewed.

\subsection{Preprocessing}

The prerequisites for the numerical simulations are considered in this section. These are the computational mesh and the settings of the solver. 


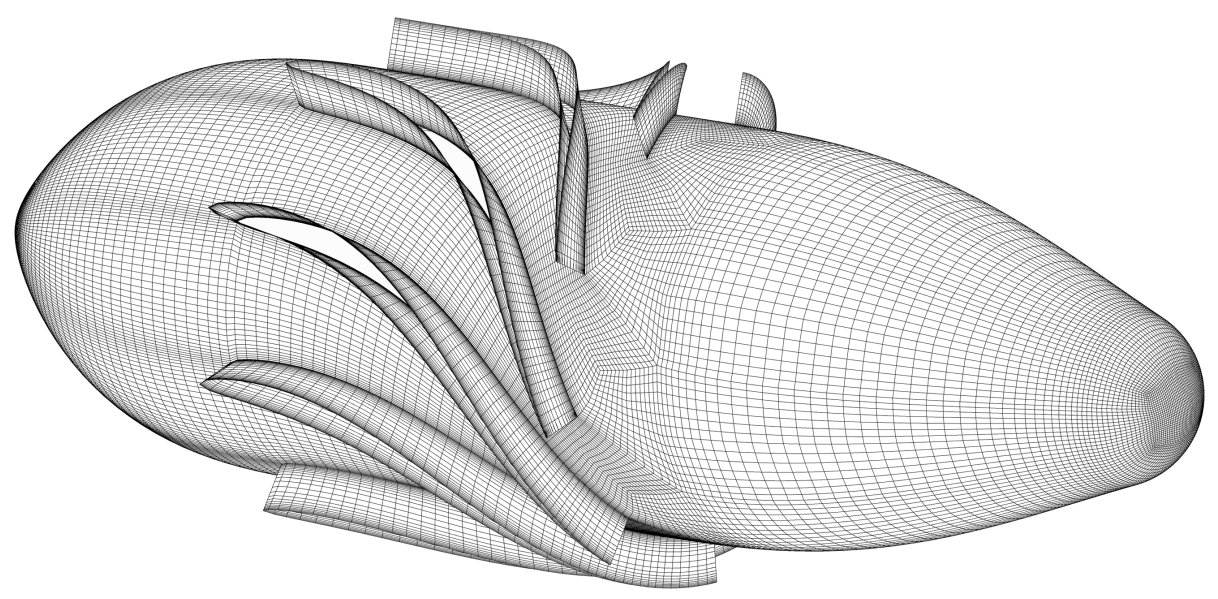

Figure 4.1: Computational surface mesh on ISE.

\subsubsection{Computational mesh}

For the flow simulations a computational mesh has to be created. This was accomplished employing ICEM CFD 14.0. The flow domain is divided into a not too large number of large hexahedral volumes, called blocks. These blocks are themselves divided into hexahedral elements. Care is taken to comply with the mesh quality requirement such as minimum and maximum angles of the hexahedral elements, variation in volume between adjacent elements and values of $y^{+}$at the walls, without using an unacceptably large number of hexahedral elements. The result of the long procedure to generate, iteratively, an adequate grid is given here, along with some meshing strategies that have been developed while generating the mesh.

In figure 4.1 the mesh on the surface of the ISE is shown. The fine mesh near the solid walls can be seen at the intersection of the vanes with the central body. This refinement is essential to capture the production of turbulent kinetic energy in the shear layer next to the walls. The large deflection of the flow by the vanes in combination with the periodicity of the geometry necessitated the introduction of a point where three, instead of four, blocks connect in order to increase the element angle to acceptable values. This point can be seen just downstream of the vanes. For the same purpose the block edges are slightly curved at various locations. At the nose and tail of the ISE all mesh lines converge, leading to a region with small elements.

A mesh without hanging nodes between the blocking interfaces is created to avoid interpolation between non-matching mesh parts. Such interpolation would lead to small wiggles in pressure and velocity and to increased computational time. To obtain such a mesh without hanging nodes the 9 blocking structures of the 9 vane passages need to connect in the center of the pipe further downstream. This leads to an O-mesh type of structure shown in figure 4.2. This figure shows a cross-section of the mesh at $z=1.0 \mathrm{~m}$. A series of numerical flow simulations has been carried out using increasingly fine meshes 
in order to determine the mesh convergence of the flow solution. It was found that for mesh convergence the element size in the center of the pipe is of prime importance. The fine mesh in the center of the pipe is required to accurately resolve the azimuthal and axial velocity distribution. It turned out that the mesh spacing in the radial and axial direction may be coarser than the mesh distribution in the azimuthal direction.

At the entrance to the pick-up tube the flow domain splits into a central cylinder leading towards the LPO and an annular region leading towards the HPO. The cut-away of the mesh in figure 4.3 shows the three-dimensional structure of the mesh in this region. The pick-up tube can also be distinguished in the figure. The mesh in the pipe can be divided into three radial sections; central, middle and near-wall. The central part contracts as it enters the pick-up tube, leading to a fine mesh in the center. It was necessary to extend the pick-up tube for $1.20 \mathrm{~m}$ in order to avoid upstream influence of imposing the location of the boundary condition on the region near the pick-up tube entrance. A C-mesh is placed around the leading edge of the pick-up tube in order to better account for the boundary layer along the wall of the pick-up tube. The middle part terminates on the leading edge of the pick-up tube, providing a high resolution mesh at this high-velocity gradient location. Again, curved block edges are employed near the pick-up tube entrance to achieve more desirable element angles. The near-wall section clearly shows the inflation layers at the pipe wall necessary to resolve the boundary layer along the pipe wall.

This mesh has $2.3 \mathrm{M}$ hexahedral elements and is used in the remainder of this research,

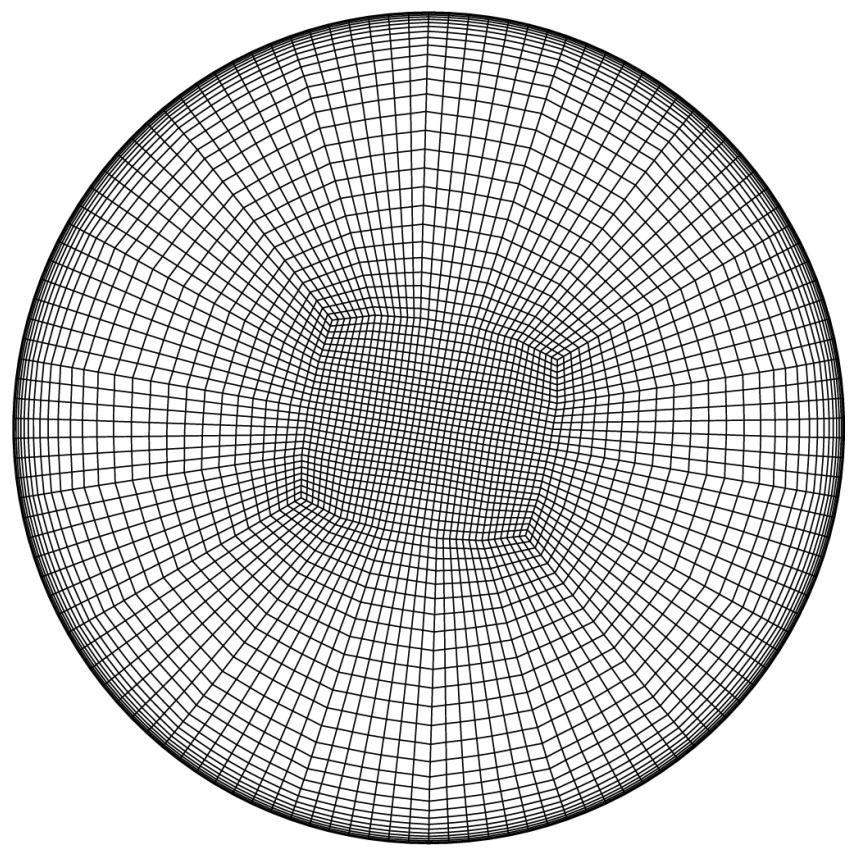

Figure 4.2: Computational mesh on cross-sectional plane in separator, $1.0 \mathrm{~m}$ downstream of ISE. 


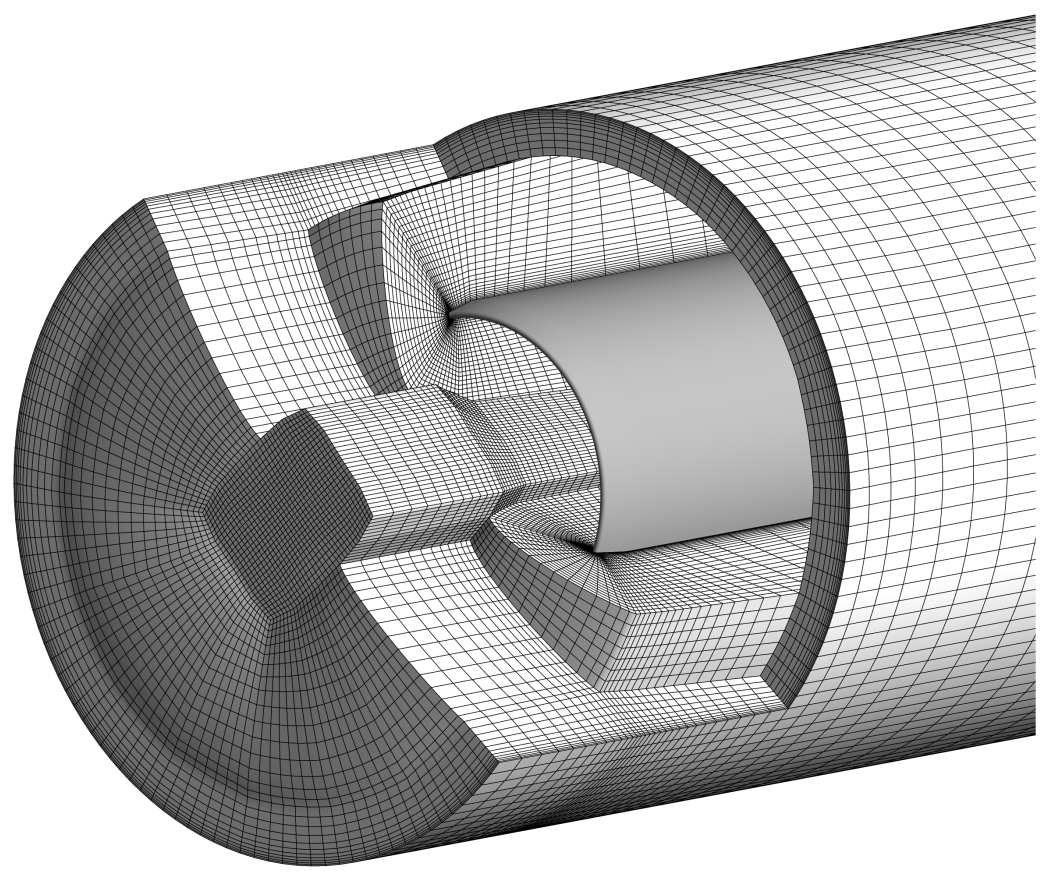

Figure 4.3: Cut-away showing three-dimensional mesh structure around entrance to the pick-up tube. Pick-up tube is shown as non-meshed surface. C-mesh surrounding pick-up tube leading edge, as well as inflation layer near pipe wall can be clearly distinguished.

unless stated otherwise. The minimum angle between grid lines is larger than $50^{\circ}$, while the maximum angle is smaller than $130^{\circ}$. The variation of the element volume of adjacent elements is smaller than a factor 1.75 for more than $99 \%$ of the elements. The maximum variation in volume is a factor 2.5 . It is advised not to let the mesh growth rates in any direction exceed 1.3. For the present three-dimensional mesh the volume variations are within this limit.

\subsubsection{Solver settings}

The boundary conditions used for the simulations of single-phase flow are shown in figure 4.4. At the inlet an axial velocity of $2 \mathrm{~m} / \mathrm{s}$ is imposed. At the LPO a mass flow is prescribed, which determines the flow split FS for single-phase flow. The velocity can vary over the cross-section of the outlet since only the total mass flow is kept at the specified value. At the HPO an area-averaged pressure is set. This combination of inlet and outlet conditions together with the solid-wall boundary conditions completely determines the flow inside the separator. The outlet conditions on LPO and HPO cannot be interchanged. Imposing a pressure condition on the LPO leads to back flow at that outlet into the flow domain. The back flow is numerical in origin and is caused by the low 

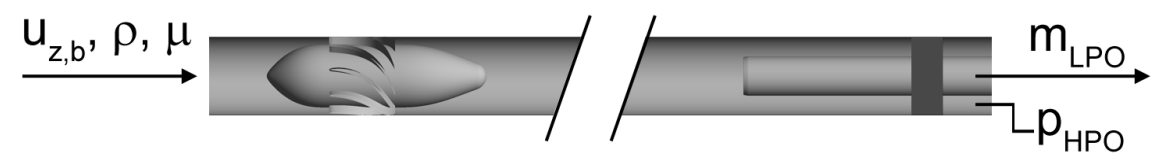

Figure 4.4: Boundary conditions used for simulations of single-phase flow.

pressure in the center of the pipe due to the swirling flow and the scaling of the pressure in order to comply with the imposed boundary condition. Moreover, the gravity vector points in the negative z-direction, that is, the fluid is flowing upwards, just like in the experimental setup at the TUD.

In the transient flow simulations the timestep $\Delta t$ is set at $0.5 \mathrm{~ms}$. The timestep has been varied to check the effect of the timestep on the flow solution. It was found that very similar results were attained for half the timestep, while increasing the timestep by a factor of two led to damping of temporal oscillations in the velocity. A smaller timestep was used when the solution had difficulty in converging.

The RMS residual criteria is set to $2.5 \times 10^{-6}$, since a convergence study of the residual revealed minimal improvement in the results for stricter criteria of the RMS residuals. Note that Ansys CFX uses a normalization procedure, however, the details of which are not disclosed in the manual.

Since the interest is not in the temporal behavior of the flow, but in the mean behavior, the results are time-averaged. In the computational domain monitoring points are placed at which the evolution of the velocity components is tracked. An operational state is defined as the state in which the velocity oscillates around a certain mean value. When this state is attained the logging of transient statistics is enabled in order to determine the time-averaged quantities. In the single-phase flow cases, it is generally sufficient for the transient statistics to run the simulations for about 1 second, that is 2000 time steps. For these transient statistics, additional solver memory needs to be allocated in the solver definition in order to prevent a memory overflow when statistics are updated after each time step.

\subsubsection{Modeling of the flow through straightener}

The flow straightener in the annular channel leading towards the HPO eliminates the swirl in the HPO. It has a honeycomb-like structure, which is very cumbersome to represent accurately in the mesh. Also, the details of the flow through the flow straightener are not of prime importance to this project.

Therefore, the flow straightener is modeled in the numerical simulations is modeled as a porous region. For this flow straightener the porosity is $\gamma=0.2667$. The reduction in flow area leads to a substantial increase in velocity. The momentum loss term $\mathbf{S}_{m}$ is given by the vector

$$
\mathbf{S}_{m}=-\mathbf{K}_{\text {loss }} \frac{\rho}{2}|\langle\mathbf{u}\rangle|\langle\mathbf{u}\rangle
$$


There is not sufficient experimental data available for calibration of the constants in the tensor $\mathbf{K}_{\text {loss }}$. Therefore, estimates have to be made and the calculated pressure drop will contain a considerable uncertainty.

It is assumed that the flow is accelerated and decelerated without losses at the start and end of the porous region, respectively. So the velocities are constant in the porous region. In addition, the Reynolds stresses are assumed to be constant as well. Since the porous region is relatively short, the viscous losses are expected to be small compared to the momentum losses caused by $\mathbf{S}_{m}$. Therefore the following approximation is found

$$
\nabla p=\mathbf{S}_{m}
$$

The pressure drop in the flow straightener is presumed to be caused by the contraction and expansion of the flow area. The losses experienced in a pipe contraction or expansion is often expressed as

$$
\Delta p=\mathbf{c}_{l o s s} \frac{\rho}{2}\langle\mathbf{u}\rangle^{2}
$$

Here $\mathbf{c}_{l o s s}$ is an empirically determined loss coefficient. For constant velocity $\langle\mathbf{u}\rangle$, the different contributions in $\mathbf{c}_{\text {loss }}$ from various pipe features can be summed to obtain the total loss coefficient $\mathbf{C}_{\text {loss }}$. Using $\nabla p=\Delta p / l_{s t r}$, where $l_{s t r}$ is the length of the flow straightener (about $0.04 \mathrm{~m}$ ), an estimate for $\mathbf{K}_{\text {loss }}$ is obtained

$$
\mathbf{K}_{\text {loss }}=\frac{\mathbf{C}_{\text {loss }}}{l_{\text {str }}}
$$

Since burrs have been removed after fabrication of the flow straightener, the edges of the contractions and expansion are assumed to be slightly rounded. The contraction and expansion are assumed to have the same loss coefficient $\mathbf{c}_{l o s s}=0.25$ [74], leading to $\mathbf{C}_{\text {loss }}=0.50$. The transverse components of the tensor $\mathbf{K}_{\text {loss }}$ are set a factor ten higher than the axial component to ensure the suppression of the radial and azimuthal velocity components. The loss tensor $\mathbf{K}_{\text {loss }}$ is then given by

$$
\mathbf{K}_{\text {loss }}=\left[\begin{array}{ccc}
120 & 0 & 0 \\
0 & 120 & 0 \\
0 & 0 & 12
\end{array}\right]\left[m^{-1}\right]
$$

As indicated by the many assumptions, this estimate of $\mathbf{K}_{\text {loss }}$ probably contains an relatively large error. However, the precise details of the flow in the flow straightener are not the focus of the project, merely the effect of eliminating the swirl needs to be accomplished.

\subsection{Flow patterns}

The predicted flow shows a distinct flow pattern, which is associated with the swirling flow imposed by the ISE. In figure 4.5 a contour plot of the time-averaged azimuthal and axial velocity components is presented for a plane through the axis of the separator. The 
highest azimuthal velocity occurs just downstream of the ISE, reaching velocities up to 16 $\mathrm{m} / \mathrm{s}$. Initially, the azimuthal velocity decreases rapidly, but further downstream the rate of decay of the swirl diminishes. The maximum azimuthal velocity at every axial location remains higher than $8 \mathrm{~m} / \mathrm{s}$. In downstream direction the location of the maximum in the radial distribution of the azimuthal velocity moves towards the center of the pipe. Near the leading edge of the pick-up tube the flow converges, leading to centrifugal accelerations as high as $600 \mathrm{~g}$. The porous region emulating the flow straightener is indicated by the dark grey ring in figure 4.5. Downstream of the flow straightener the azimuthal velocity component is zero.

The radial distribution of the azimuthal velocity is as expected. On the contrary, the distribution of the axial velocity can not be readily explained. Over most of the length of the pipe an annular region of reversed flow is observed. With a positive axial velocity near the wall and in the center, the radial distribution of the axial velocity is W-shaped. In the region of the afterbody of the ISE the flow detaches from the surface of the ISE, this is caused by the swirling flow. The location of this detachment line depends on the amount of swirl, as will be discussed later on.

Although obscured by the time-averaging, the flow is quite unsteady near the center of the pipe. Also obscured by the time-averaging is the mild precession of the vortex core. This vortex core precession is a low-amplitude time-dependent motion of a helical vortex core around the geometrical axis of the pipe. The vortex core precession is strongest just aft of the ISE. The unsteadiness decreases in the downstream direction. The latter may be attributed to the pick-up tube, which appears to have a stabilizing effect on the flow.

\subsubsection{Flow reversal}

Reversed axial flow in strongly swirling pipe flow has been reported before in the literature $[57,29]$. Often the axial velocity distribution shows a local minimum in the center of the pipe, resulting in a V-shaped velocity distribution. This distribution is sometimes explained by the adverse pressure gradient along the pipe axis [16]. The swirl decreases

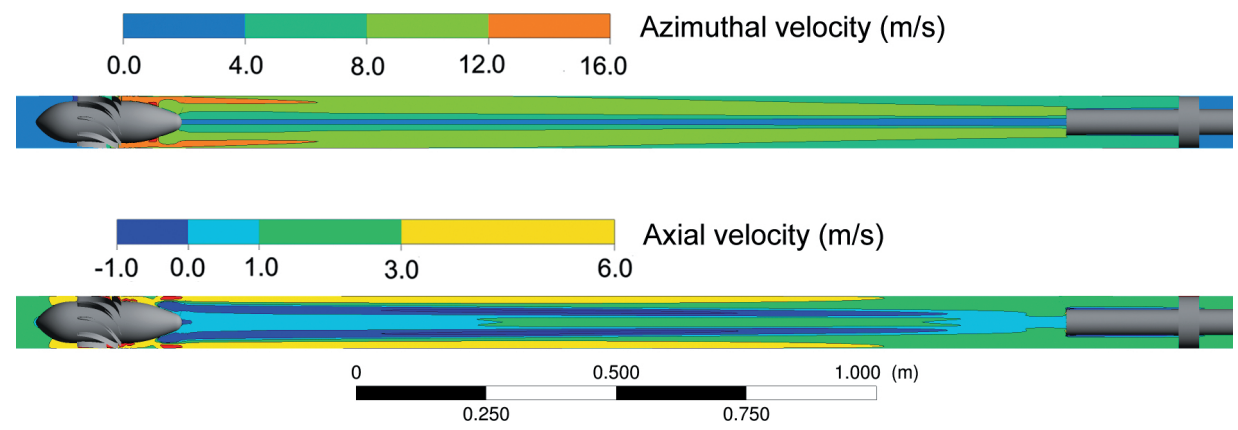

Figure 4.5: Contours of time-averaged azimuthal (top) and axial velocity (bottom) on the plane $x=0 \mathrm{~m}$ through the axis of separator. Annular reversed flow region is visible. Inlet bulk velocity is $2 \mathrm{~m} / \mathrm{s}, \mathrm{HPO}$ reference pressure is $0 \mathrm{~Pa}, \dot{m}_{L P O}=5.03 \mathrm{~kg} / \mathrm{s}$. 


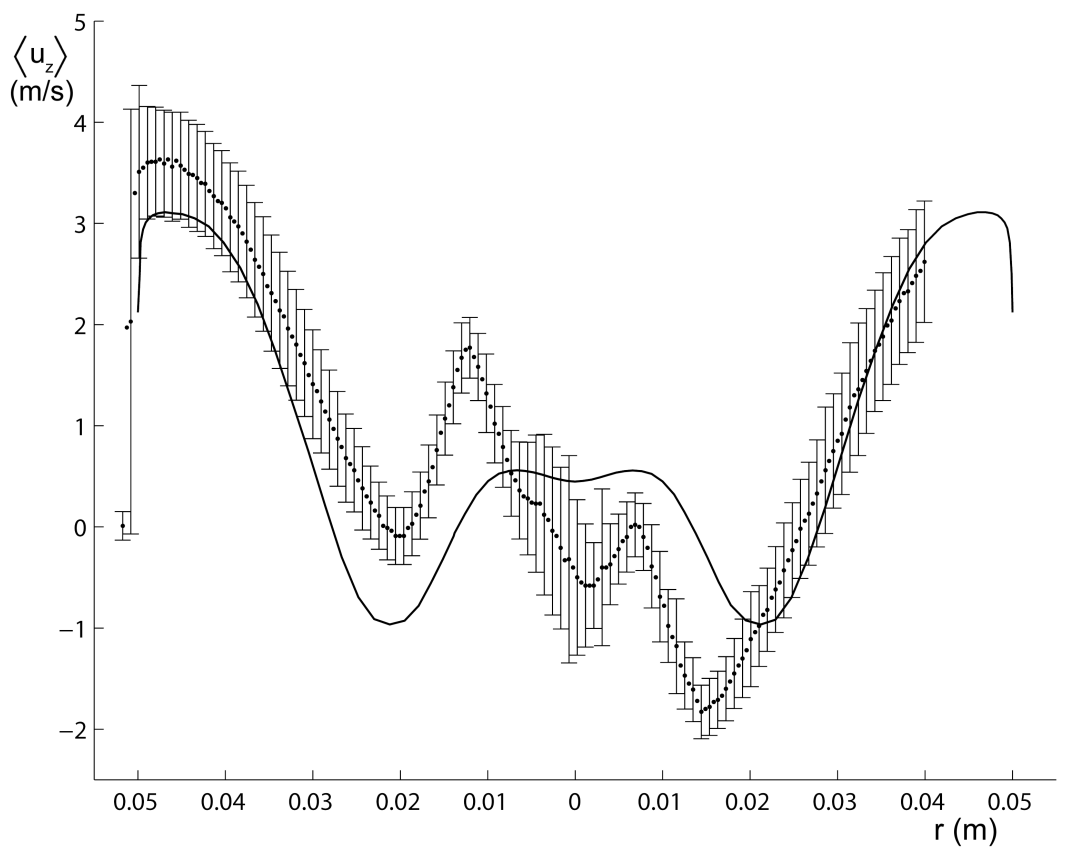

Figure 4.6: Comparison of calculated (solid) and measured (dotted) radial distribution of the time-averaged axial velocity along a line through the axis of the separator at $z=0.44 \mathrm{~m}$ downstream of ISE. Inlet bulk velocity in simulation is $1.5 \mathrm{~m} / \mathrm{s}\left(42.2 \mathrm{~m}^{3} / \mathrm{h}\right)$. Flow rate in experiment is $43 \mathrm{~m}^{3} / \mathrm{hr}$. Error bars indicate standard deviation in experimental results. Experimental results by Laurens van Campen, TUD.

in the downstream direction due to friction. As a consequence, the difference in pressure between the center and the wall will also decrease in the axial direction $z$. This results in an increase in pressure in the center, while near the wall the pressure decreases. This mechanism gives not the complete explanation for all instances of swirling flow in which a $\mathrm{V}$-shaped distribution of the axial velocity is observed. For the current situation, showing a double reversal or $\mathrm{W}$-shaped distribution of the axial velocity, it fails to reveal the cause of the observed flow pattern.

The W-shaped profile has been observed before by Mattner et al. [75] and Brücker [13] for swirling pipe flow at very high swirl numbers. Mattner associated this flow pattern with the phenomena of vortex breakdown. Vortex breakdown can be described as a sudden structural change in the flow pattern in swirling flow. It is commonly seen in swirling pipe flow, wing trailing vortices, vortical flow generated by delta wings and inside combustion chambers. Although vortex breakdown has a long history of investigation, still little agreement exists on the complete explanation of the phenomenon. Various theories have been put forward to explain vortex breakdown. The critical state theory $[9,106]$ states, roughly, that vortex breakdown occurs at the transition between a state in which infinitesimal waves can only travel downstream into a state in which upstream propagation 


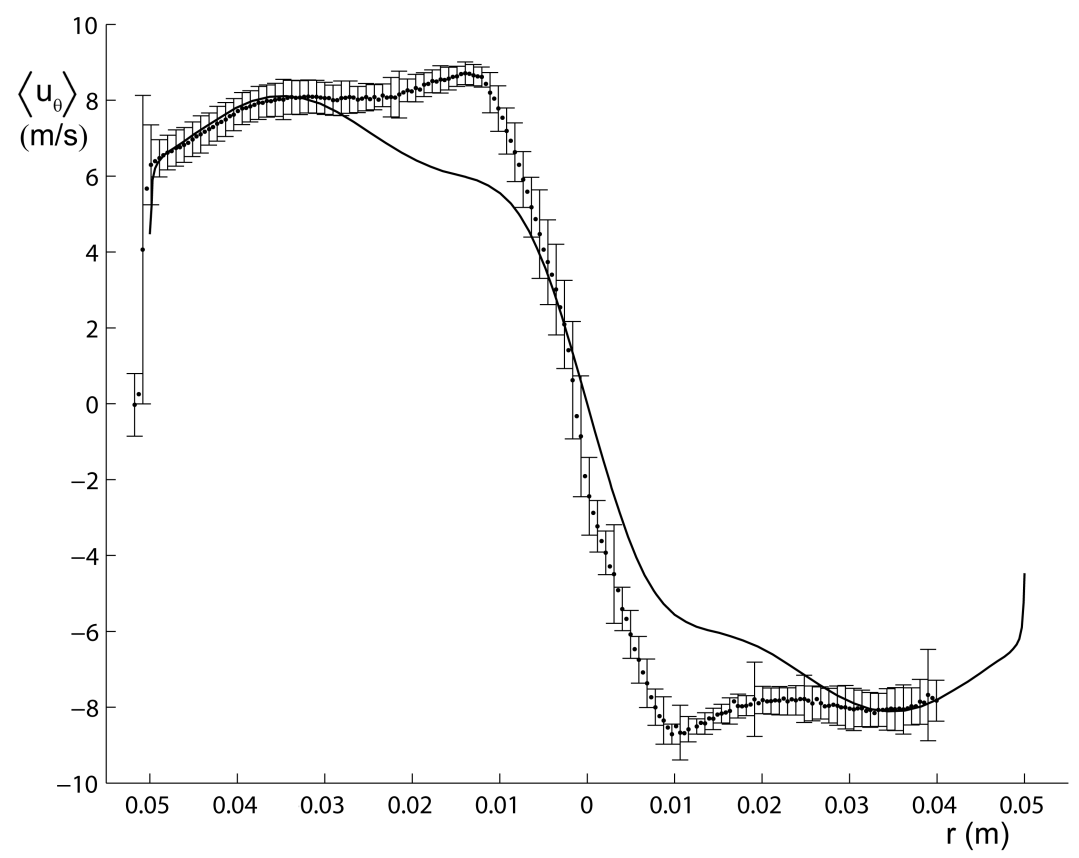

Figure 4.7: Comparison of calculated (solid) and measured (dotted) radial distribution of the time-averaged azimuthal velocity along a line through the axis of the separator at $z=0.44 \mathrm{~m}$ downstream of ISE. Inlet bulk velocity in simulation is $1.5 \mathrm{~m} / \mathrm{s}\left(42.2 \mathrm{~m}^{3} / \mathrm{h}\right)$. Flow rate in experiment is $43 \mathrm{~m}^{3} / \mathrm{hr}$. Error bars indicate standard deviation in experimental results. Experimental results by Laurens van Campen, TUD.

is possible. Another explanation may be provided by hydrodynamical instability, for instance Leibovich and Stewartson [66] supply a criterion below which the columnar vortex flow becomes unstable. The existence of multiple flow solutions at a certain swirl number and the flow transition between these solutions offers yet another way to gain insight into vortex breakdown [91]. Also attempts have been made to relate different theories in order to arrive at a more complete mechanism of vortex breakdown [80]. More mechanisms have been investigated [113], however a thorough examination of vortex breakdown is beyond the scope of this thesis. For a more comprehensive treatment of this phenomenon the reader is referred to literature reviews by for instance Lucca-Negro [80] and Leibovich [65].

\subsubsection{Validation by comparison with experimental data}

A comparison between the numerical and experimental results along a line through the axis of the separator at $z=0.44 \mathrm{~m}$ is shown in figures 4.6 and 4.7. The results are time-averaged velocities and the error bars indicate the standard deviation in the measurements. The LDA measurements have been provided by Laurens van Campen from 
the TUD. The flow rate in the experiment is $43 \mathrm{~m}^{3} / \mathrm{h}$. In the simulation the inlet velocity is $1.5 \mathrm{~m} / \mathrm{s}$, giving a flow rate of $42.4 \mathrm{~m}^{3} / \mathrm{h}$.

The experimental results show asymmetries, which are steady in time. These are caused by asymmetries in the experimental setup, presumably bends in the piping downstream of the LPO and small misalignment of the various parts in the setup. The asymmetry of the flow is greatly reduced when a flow straightener is placed upstream of the pick-up tube, preventing swirling flow in the pipe bend further downstream. Moreover, despite efforts to remove all air and oil from the incoming flow, a very thin kernel of lighter phase was present at the very center of the pipe. This slightly influences the flow in the center and affects the accuracy of the measurements.

Besides the asymmetries, good agreement on the flow pattern is attained. The experimental results show the $\mathrm{W}$-shaped distribution of the axial velocity with the annular reversed flow region. The comparison confirms that the computed flow pattern is also seen in reality and it is not some numerical artifact stemming from the modeling.

The radial distribution of the azimuthal velocity follows the same trend in the numerical simulation and the experiment, however, the experiment shows higher velocities near the center.

Overall, the accuracy of the result of the numerical simulations based on single-phase RANS is satisfactory and therefore these simulations are used for further study of the flow in the separator.

\subsubsection{Evaluation design approach}

In chapter 3, an engineering approach has been followed to design the ISE. This design approach is now evaluated by comparing the results from the CFD simulations to the estimated design requirements.

Various assumptions have been made in the derivation of the required azimuthal velocity at the trailing edge of the vanes. In equation (3.4) the azimuthal velocity is approximated by a core with a solid-body rotation and a constant velocity in the outer part. The matching of these velocity distributions is at $R_{C}=0.25 R$. The simulations confirm that this is a reasonable approximation of the velocity field in the design phase. However, a more accurate description can be attained in future work by using a potential-flow distribution for the azimuthal velocity in the outer part of the radial distribution.

Moreover, in deriving equation (3.9) it has been assumed that the droplets are subject to Stokes' drag and that turbulent dispersion of droplets is neglected. The range of validity of Stokes' drag stops when the incoming feed can no longer be seen as a dilute dispersion. Furthermore, the effect of turbulence was shown to be significant in exploratory droplet tracking calculations. Both effects cannot be predicted by simple engineering approaches. The best way is to account for these effects by using some safety margin in the design. The loss factor $\epsilon_{S W}$ should give the decrease is angular momentum caused by the transition from the flow in the vane section to the swirling pipe flow, that is, the decrease not accounted for by the viscous shear at the solid surfaces. The design velocity at the trailing edge of the vane scales inversely with this loss factor $\epsilon_{S W}$. Analysis of the numerical results shows that there is no sudden, large drop in angular momentum and that, also 
very close to the ISE, the decrease in angular momentum shows an exponential decay. In the work of Dirkzwager [29], the first measurement location showed a large decrease in angular momentum compared to the design specification of his axial hydrocyclone. To capture this effect the loss factor was introduced in the design phase. However, this first measurement location was 10 pipe diameters downstream of the swirl element. The numerical simulations show a very similar decrease in angular momentum of $46 \%$ over the same distance. Such losses are already accounted for in the exponential decay of the azimuthal velocity given in equation (3.6). In retrospect it was wrongfully assumed that these losses were caused by transitional effects. The advice is to not use the loss factor $\epsilon_{S W}$ for future design studies.

Furthermore, the axial velocity in the calculation of the angular momentum was assumed constant over the cross-section. Simulations show that this is not the case. It is, however, difficult to account for the complex flow patterns in these engineering design methods.

Inspection of the flow around the vanes shows that flow separation does not occur. The azimuthal velocity, averaged over a cross-flow plane just aft of the vanes, is $13.6 \mathrm{~m} / \mathrm{s}$. This corresponds to an averaged flow deflection of $69^{\circ}$ measured with respect to the axial direction. This is very close to the angle of the camber line of $68.1^{\circ}$ as given in section 3.2.2. Therefore, the assumptions made in the vane design process and the vane design procedures, that is the use of a potential flow method and a two-dimensional cascade geometry, are considered appropriate. 


\subsection{Velocity distributions}

A more detailed view of the flow field can be obtained by examining the radial distribution of the axial and azimuthal velocity components. Figures 4.8, 4.9 and 4.10 show the evolution with axial distance from the ISE of the time-averaged axial velocity. In the pipe downstream from the ISE, the characteristic W-shape is seen with a small dip at the center of the pipe. Halfway the pipe, a distinct trend can be seen; the annular reversed flow region moves inward while its minimum velocity slowly become less negative. Simultaneously the axial velocity near the pipe wall decreases, while the axial velocity in the center of the pipe increases. Also the dip in the axial velocity distribution in the center of the pipe becomes more distinct in downstream direction.

The flow changes when it approaches the pick-up tube at $z=1.70 \mathrm{~m}$. The trends seen in the annular reversed flow region continue, while the axial velocity in the center starts to decrease. This yields a smoother velocity distribution in which the annular reversed flow region dissolves, see figure 4.9 .

The last shown axial location at $z=1.75 \mathrm{~m}$ is just inside the pick-up tube. The discontinuity in the velocity distribution is due to the wall of the pick-up tube, which has an inner and outer radius of 23 and $25 \mathrm{~mm}$, respectively. In the pick-up tube a local maximum forms at the center, replacing the dip, see figure 4.10. The cause of this is not easily found and is likely to be related to the change in swirl.

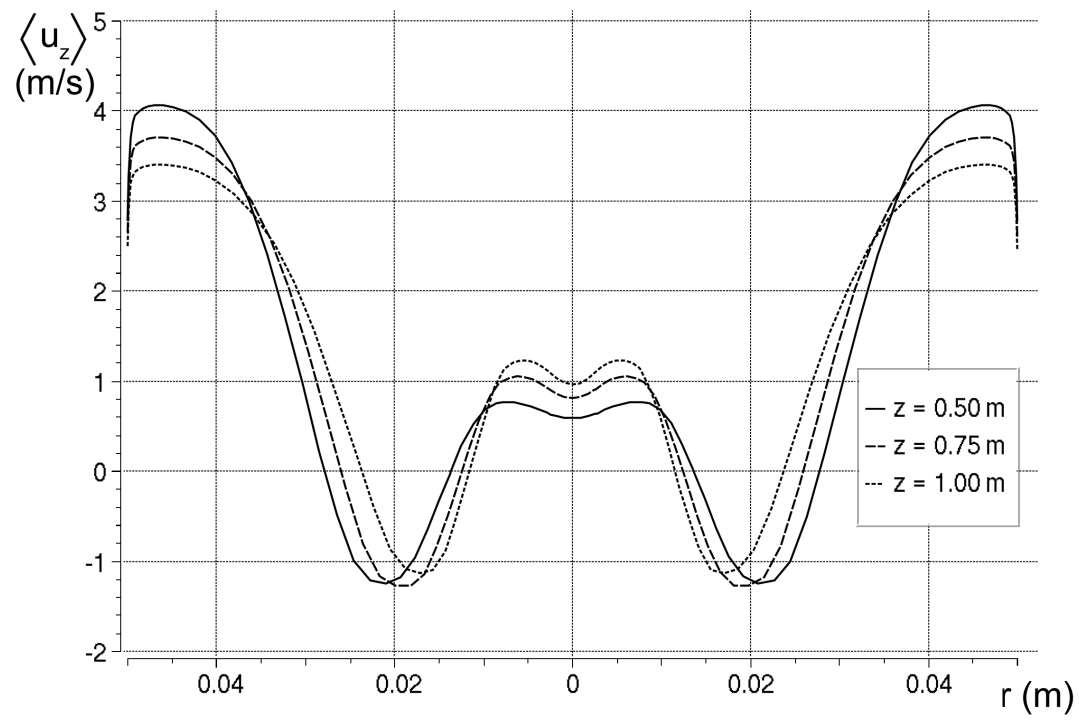

Figure 4.8: Radial distribution of the time-averaged axial velocity on a line through the center of the pipe at $z=0.50,0.75$ and $1.00 \mathrm{~m}$. Inlet bulk velocity is $2 \mathrm{~m} / \mathrm{s}, \mathrm{HPO}$ reference pressure is $0 \mathrm{~Pa}, \dot{m}_{L P O}=5.03 \mathrm{~kg} / \mathrm{s}$. 


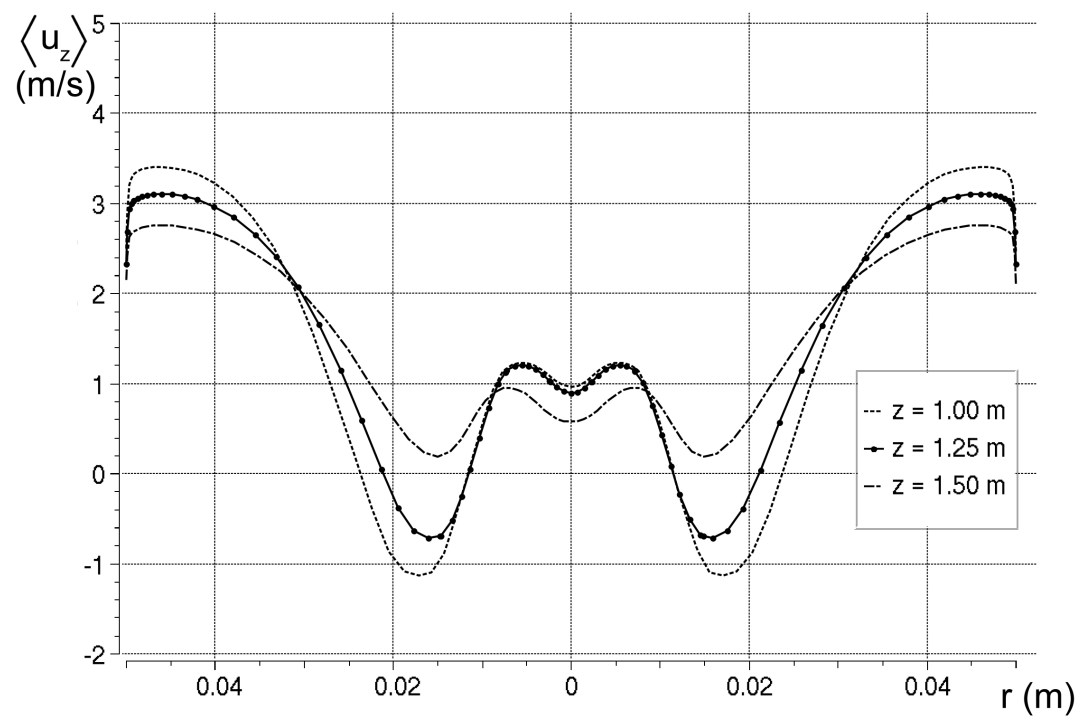

Figure 4.9: Radial distribution of the time-averaged axial velocity on a line through the center of the pipe at $z=1.00,1.25$ and $1.50 \mathrm{~m}$. Inlet bulk velocity is $2 \mathrm{~m} / \mathrm{s}, \mathrm{HPO}$ reference pressure is $0 \mathrm{~Pa}, \dot{m}_{L P O}=5.03 \mathrm{~kg} / \mathrm{s}$.

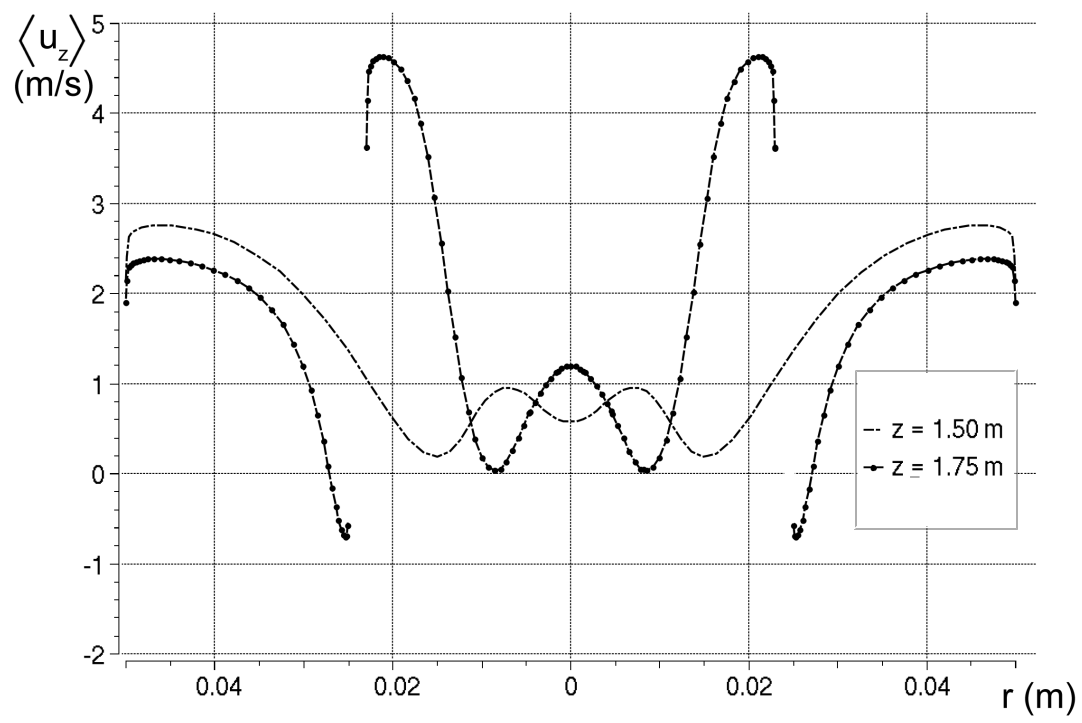

Figure 4.10: Radial distribution of the time-averaged axial velocity on a line through the center of the pipe at $z=1.50$ and $1.75 \mathrm{~m}$. Inlet bulk velocity is $2 \mathrm{~m} / \mathrm{s}$, HPO reference pressure is 0 $\mathrm{Pa}, \dot{m}_{L P O}=5.03 \mathrm{~kg} / \mathrm{s}$. 
The azimuthal velocity distribution in the pipe is plotted in figure 4.11. The azimuthal velocity distribution can be divided into three regions. Near the wall a thin boundary layer is observed. Further away from the wall a region exists with high azimuthal velocities and a distribution similar to that of a potential-flow vortex. Near the center the distribution resembles that of a solid-body rotation, although the gradient is not constant. The velocity at the wall does not become zero since the meshing is too coarse to fully resolve the boundary layer. Instead wall functions are employed and the $y^{+}$at the outer wall varies from 90 near the location of the detachment line on the ISE to 40 at the end of the separator near the flow straightener. This is the range of $y^{+}$for which the log law applies.

In the potential flow vortex region the velocity decreases with axial distance due to friction. After an initial decline the maximum azimuthal velocity increases further downstream, while the location of the maximum moves towards the pipe axis. In the center of the solid-body rotation core, the slope of the azimuthal velocity decreases slightly in the downstream direction, while it increases at the edge of this core.

Near the ISE a dent in the azimuthal velocity distribution can is seen around $r=0.02 \mathrm{~m}$. This is due to the specific flow around and just downstream of the ISE. Close to the ISE there is actually a local minimum in the azimuthal velocity, however, at $z=0.50 \mathrm{~m}$ this minimum in the distribution is already smoothed out.

In figure 4.12 the azimuthal velocity distribution in the pick-up tube is given. Again the location of maximum velocity moves inward with the downstream direction. A relatively

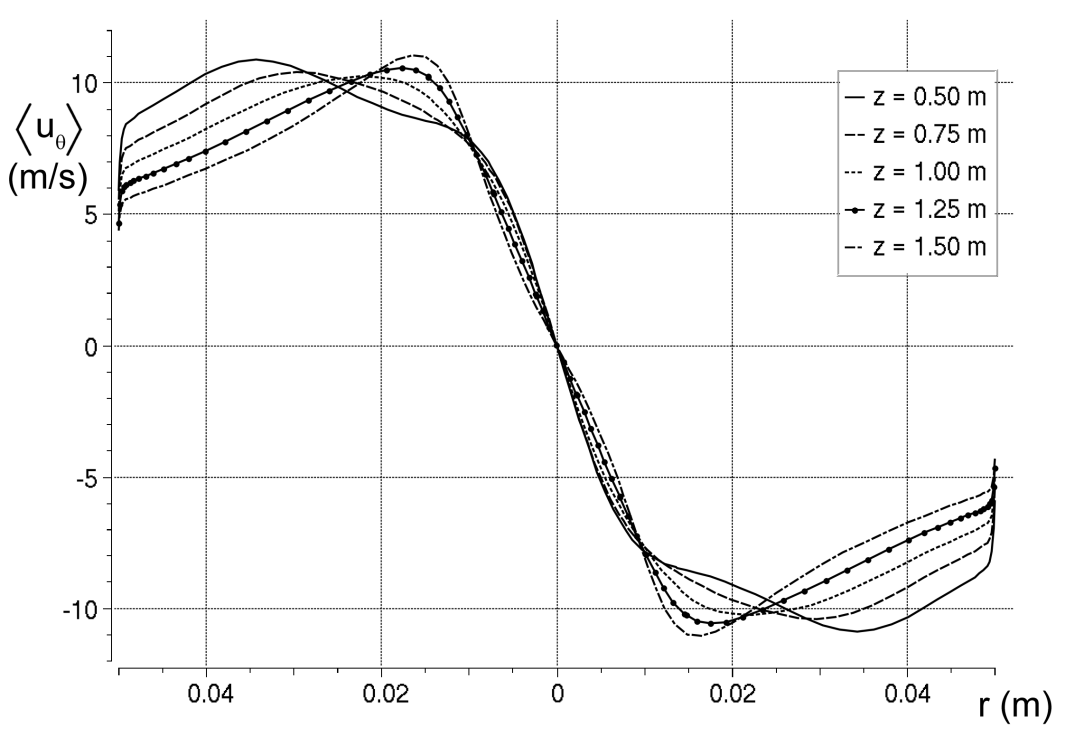

Figure 4.11: Radial distribution of the time-averaged azimuthal velocity on a line through the center of the pipe at $z=0.50,0.75,1.00,1.25$ and $1.50 \mathrm{~m}$. Inlet bulk velocity is $2 \mathrm{~m} / \mathrm{s}$, HPO reference pressure is $0 \mathrm{~Pa}, \dot{m}_{L P O}=5.03 \mathrm{~kg} / \mathrm{s}$. 
rapid decrease is seen in the maximum velocity due to friction at the wall of the pick-up tube. In the center the velocity gradient now increases in downstream direction. This is different from the trend observed in the pipe. However, this steepening of the velocity gradient in downstream direction has been reported before by Kitoh [57] and Dirkzwager [29]. At $z=2.00 \mathrm{~m}$ the flow has passed the flow straightener, which effectively sets the azimuthal velocity component equal to zero in the HPO.

Figure 4.13 shows the evolution of the maximum time-averaged azimuthal velocity in the cross-flow plane as function of $z$. A gradual decrease in maximum azimuthal velocity is observed up to $z=1.00 \mathrm{~m}$, after which it increases until the entrance to the pick-up tube is reached. As the flow enters the pick-up tube a sharp peak in maximum azimuthal velocity is seen. Inside the pick-up tube the maximum azimuthal velocity is quickly reduced.

The radial velocity component is very small compared to the other two components. The radial component of the velocity is smaller than $2 \%$ of the axial bulk velocity $u_{z, b}$ except for regions near the ISE and the pick-up tube.

Another way to examine the distribution of the azimuthal velocity is to plot the distribution of the axial vorticity $\omega_{z}$

$$
\omega_{z}=\frac{1}{r} \frac{\partial}{\partial r}\left(r u_{\theta}\right)
$$

Near the wall of the pipe the azimuthal velocity rapidly decreases with increasing radius, therefore a large negative axial vorticity $\omega_{z}$ will be present in this region. Further away from the wall a region with a potential-flow like distribution of the azimuthal velocity is

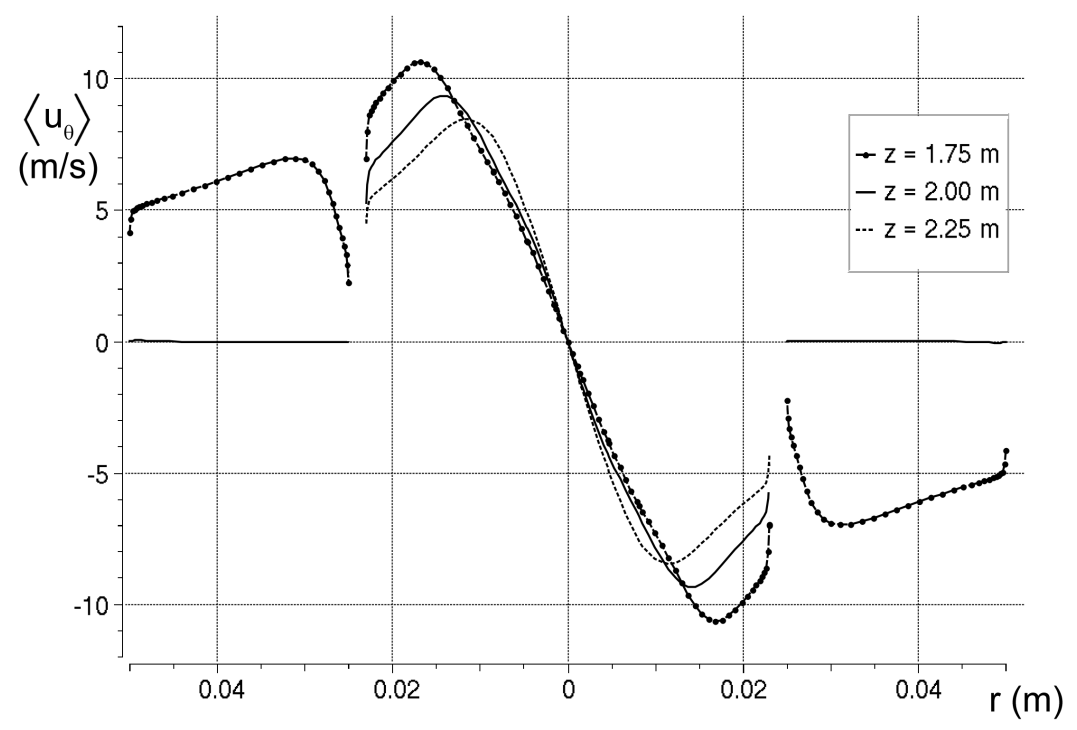

Figure 4.12: Radial distribution of the time-averaged azimuthal velocity on a line through the center of the pipe at $z=1.75,2.00$ and $2.25 \mathrm{~m}$. Inlet bulk velocity is $2 \mathrm{~m} / \mathrm{s}$, HPO reference pressure is $0 \mathrm{~Pa}, \dot{m}_{L P O}=5.03 \mathrm{~kg} / \mathrm{s}$. 


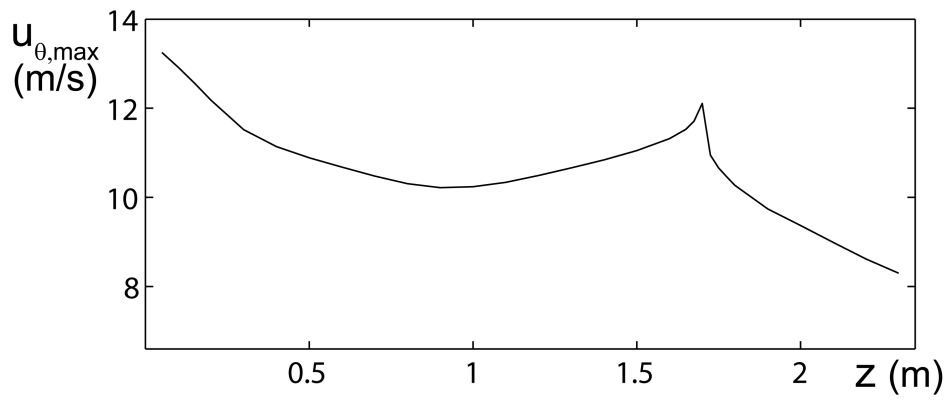

Figure 4.13: Maximum time-averaged azimuthal velocity in cross-flow plane as function of $z$. Inlet bulk velocity is $2 \mathrm{~m} / \mathrm{s}, \mathrm{HPO}$ reference pressure is $0 \mathrm{~Pa}, \dot{m}_{L P O}=5.03 \mathrm{~kg} / \mathrm{s}$.

identified. In such a region the azimuthal velocity varies with the radius as $u_{\theta}(r) \propto 1 / r$, this yields a region of zero axial vorticity. Near the center of the pipe solid-body rotationlike distributions of the azimuthal velocity with different slopes are seen. In these regions the azimuthal velocity varies as $u_{\theta}(r) \propto r$ and so $\omega_{z}$ is piece-wise constant in these regions.

Figure 4.14 shows the radial distribution of the time-averaged axial vorticity $\omega_{z}$ on a line through the center of the pipe at $z=0.50,1.00$ and $1.50 \mathrm{~m}$. Near the wall a thin region of large negative axial vorticity is indeed seen. Note that the vertical axis of the figure is clipped at $\omega_{z}=-10001 / \mathrm{s}$ for convenience. The actual potential-flow region is smaller than expected based on figure 4.11, as appreciable axial vorticity levels are observed in the region $0.02<r<0.04 \mathrm{~m}$. However, the distribution becomes more potential-flowlike in the downstream direction. In the center the distribution of axial vorticity shows a

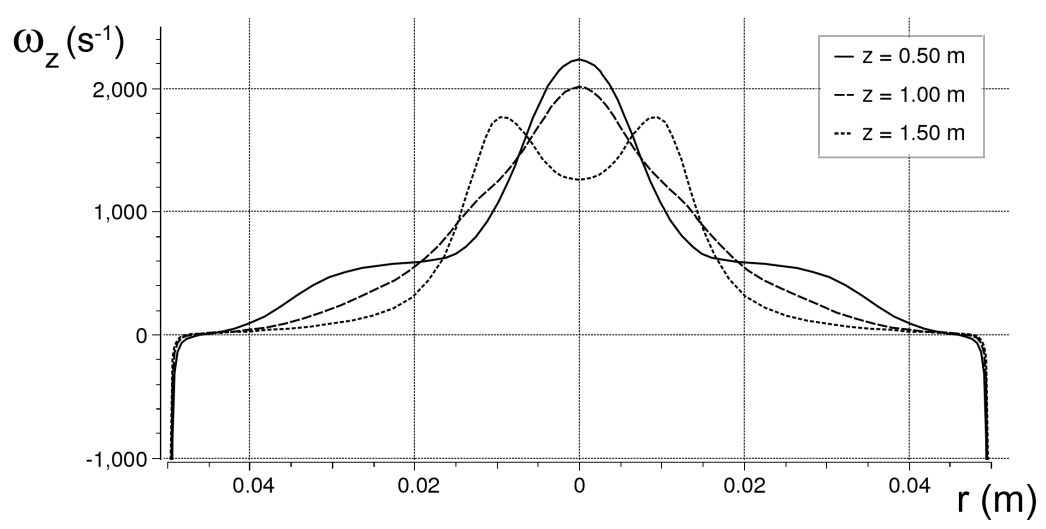

Figure 4.14: Radial distribution of the time-averaged axial vorticity on a line through the center of the pipe at $z=0.50,1.00$ and $1.50 \mathrm{~m}$. Inlet bulk velocity is $2 \mathrm{~m} / \mathrm{s}, \mathrm{HPO}$ reference pressure is $0 \mathrm{~Pa}, \dot{m}_{L P O}=5.03 \mathrm{~kg} / \mathrm{s}$. 
more parabolic behavior, rather than a constant value. At $z=1.50 \mathrm{~m}$, the axial vorticity distribution in the center corresponds to the lower slope of the azimuthal velocity at the pipe axis and a steeper slope at large radius, as is seen in figure 4.11. The variation in axial vorticity indicates a continuously varying slope of the azimuthal velocity distribution and therefore the distribution of the azimuthal velocity only approximates the solid-body rotation and potential-flow velocity distributions.

\subsection{Pressure distribution}

The absolute pressure $p$ can be split into three contributions

$$
p=\hat{p}+\rho g(h-z)+p_{0} .
$$

Here $p_{0}$ is a reference pressure, set to zero in this work for convenience. At the reference height $h$, the hydrostatic pressure is zero relative to $p_{0}$. The gradient of the hydrostatic pressure component $\rho g(h-z)$ cancels out with the gravity term $\rho g$ and only $\hat{p}$ influences the velocity distribution. Moreover, in the current application both $p_{0}$ and $h$ are not uniquely determined, which leads to an arbitrary absolute pressure. Therefore, only the pressure component $\hat{p}$ will be discussed and the hat is subsequently dropped for convenience. Figure 4.15 shows the time-averaged radial pressure distribution at $z=0.50,1.00$ and $1.50 \mathrm{~m}$. Negative pressures are seen because the reference pressure is set at the HPO

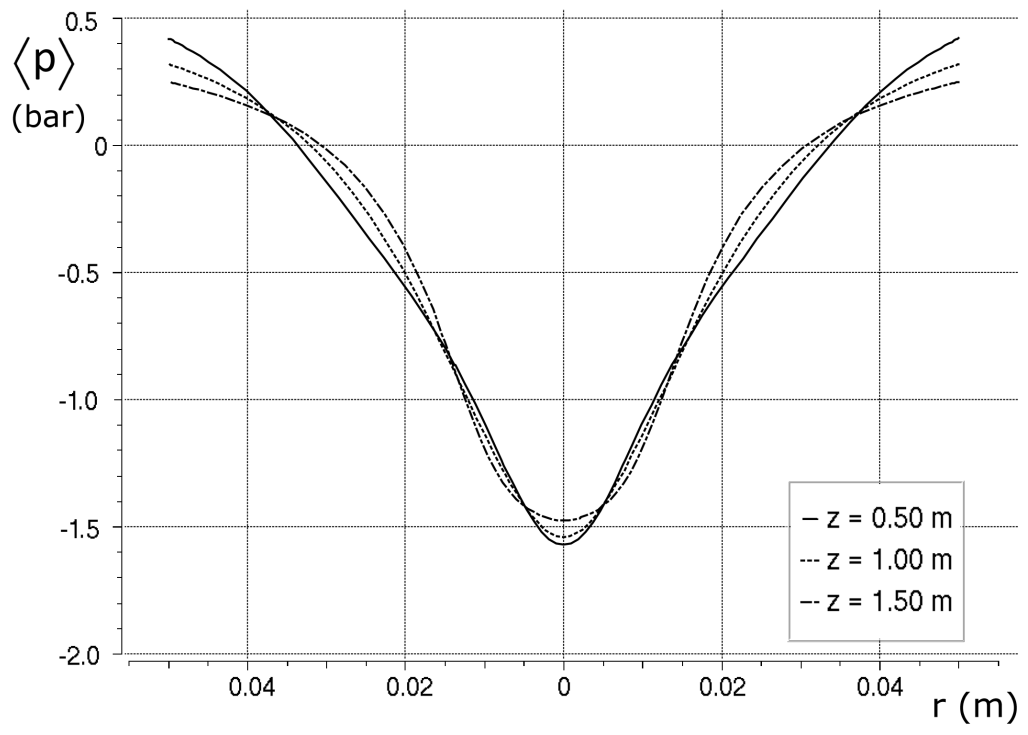

Figure 4.15: Radial distribution of the time-averaged static pressure on a line through the center of the pipe at $z=0.50,1.00$ and $1.50 \mathrm{~m}$. Inlet bulk velocity is $2 \mathrm{~m} / \mathrm{s}, \mathrm{HPO}$ reference pressure is $0 \mathrm{~Pa}, \dot{m}_{L P O}=5.03 \mathrm{~kg} / \mathrm{s}$. 
to $0 \mathrm{~Pa}$. In the region just outside the center $(r>5 \mathrm{~mm})$, the radial pressure gradient increases in downstream direction, which corresponds to the increase in the maximum azimuthal velocity gradient seen in figure 4.11. The pressure difference between the wall and the center is declining from 2.2 bar just downstream of the ISE to 1.7 bar just upstream of the pick-up tube.

From the distributions in figure 4.15 it can be inferred that the axial gradient of the pressure changes sign three times from the center to the wall. This can be matched with the behavior of the distribution of the axial velocity. The negative axial pressure gradient near the wall and in the region $5 \mathrm{~mm}<r<15 \mathrm{~mm}$, corresponds with a positive axial velocity. The annular region of reversed flow is seen at $15 \mathrm{~mm}<r<35 \mathrm{~mm}$. In the very center the pressure gradient is also positive. Although the axial velocity remains positive there, the velocity dip deepens as can be observed in figure 4.8 , that is the flow decelerates.

The time-averaged pressure along a line on the pipe wall at $r=0.05 \mathrm{~m}$ can be seen in figure 4.16. The pressure shows an exponential-like decay. The brief pressure increase at $z=1.70 \mathrm{~m}$ is caused by the presence of the pick-up tube entrance.

The time-averaged pressure along the axis of the pipe is shown in figure 4.17. From $z=$ $0.50 \mathrm{~m}$ the pressure rises to a maximum at about $z=1.60 \mathrm{~m}$, after which the pressure decreases rapidly as the flow enters the pick-up tube indicating an increase of the swirl of the flow.

A relevant separator characteristic is the pressure drop between the inlet and the outlets. Since the pressure drop over the flow straightener is not accurate because of the simple model used for the losses in that region, the area-averaged pressure at the HPO is evaluated at the annular plane half a pipe diameter upstream of the flow straightener. For fair comparison the area-averaged pressure at the LPO is evaluated at the same axial location. The pressure drop between the inlet and the HPO is 1.6 bar, while the pressure drop

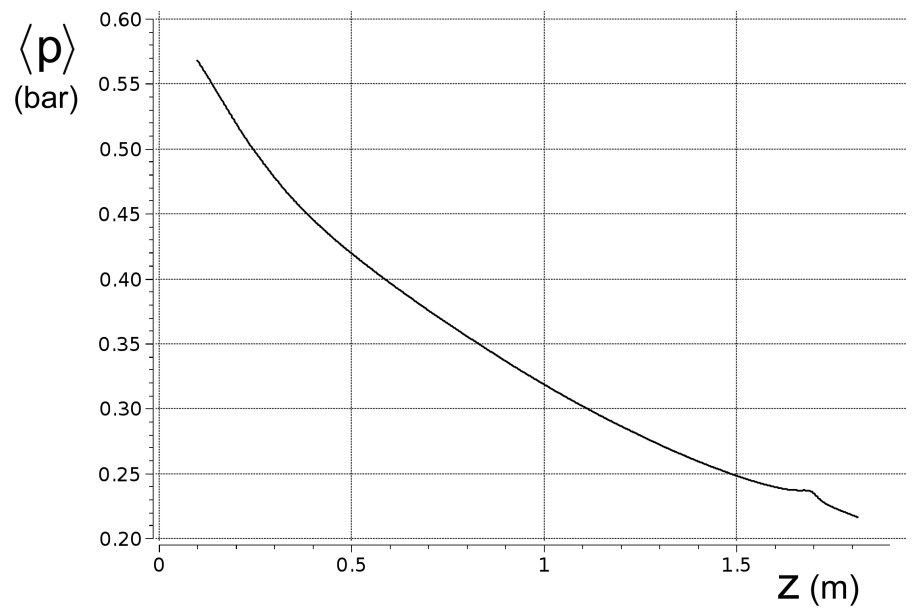

Figure 4.16: Time-averaged static pressure along a line on the pipe wall at $r=0.05 \mathrm{~m}$. Inlet bulk velocity is $2 \mathrm{~m} / \mathrm{s}, \mathrm{HPO}$ reference pressure is $0 \mathrm{~Pa}, \dot{m}_{L P O}=5.03 \mathrm{~kg} / \mathrm{s}$. 


\begin{tabular}{|l||c|c|c|}
\hline & Inlet - ISE & Inlet - HPO & Inlet - LPO \\
\hline \hline$\Delta\langle\bar{p}\rangle$ (bar) & 1.8 & 1.6 & 2.5 \\
$\Delta\left\langle\bar{p}_{\text {tot }}\right\rangle$ (bar) & 1.1 & 1.4 & 2.1 \\
\hline
\end{tabular}

Table 4.1: Area-averaged static and total pressure difference $\Delta\langle\bar{p}\rangle$ and $\Delta\left\langle\bar{p}_{\text {tot }}\right\rangle$ between inlet and outlets.

between the inlet and LPO is 2.5 bar. The ISE generates the swirling flow at the expense of a decrease in static pressure of 1.8 bar. This is computed by taking the area-averaged pressure over a cross-sectional plane $10 \mathrm{~mm}$ upstream and downstream of the ISE.

The energy losses in the separator can be made clear by examining the time-averaged total pressure $\left\langle p_{t o t}\right\rangle$ area-averaged over the cross-flow plane:

$$
\left\langle\bar{p}_{t o t}\right\rangle=\frac{1}{\pi R^{2}} \int_{0}^{R}\left\langle p_{t o t}\right\rangle 2 \pi r d r
$$

The drop in total pressure $\left\langle\bar{p}_{\text {tot }}\right\rangle$ between inlet and the HPO is 1.4 bar, while between the inlet and the LPO 2.1 bar of $\left\langle\bar{p}_{\text {tot }}\right\rangle$ is lost. Inside the pick-up tube high losses are observed due to the high velocities in the narrow pipe. The decrease in the kinetic energy can also be deduced from figure 4.12. Comparing the total and static pressure drops between inlet and outlets is clear that due to high losses only limited gains can be made by pressure recovery, for instance by de-swirling, in downstream piping. The decrease in $\left\langle\bar{p}_{t o t}\right\rangle$ over the ISE is 1.1 bar. So only about $40 \%$ of the static pressure drop is converted into kinetic energy. The difference in static pressure, averaged over a plane just after the

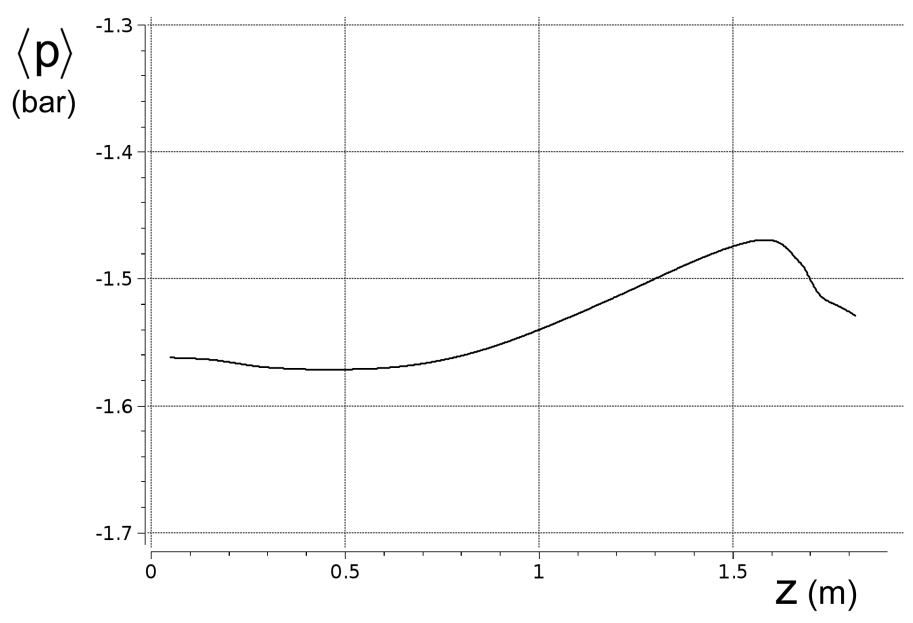

Figure 4.17: Time-averaged static pressure along the axis of the pipe. Inlet bulk velocity is 2 $\mathrm{m} / \mathrm{s}, \mathrm{HPO}$ reference pressure is $0 \mathrm{~Pa}, \dot{m}_{L P O}=5.03 \mathrm{~kg} / \mathrm{s}$. 


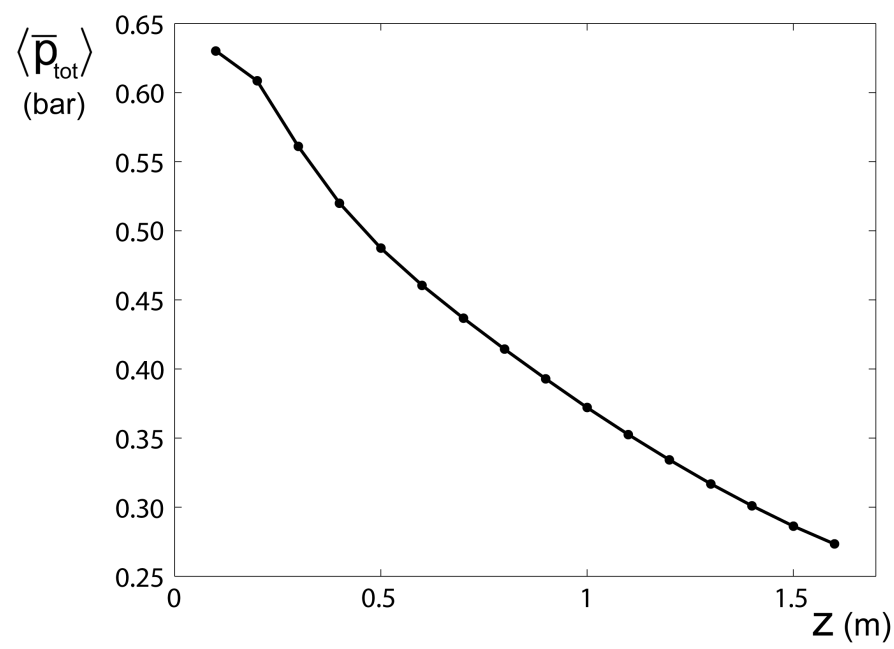

Figure 4.18: Time-averaged total pressure, area-averaged over cross-flow plane as function of $z$. Inlet bulk velocity is $2 \mathrm{~m} / \mathrm{s}$, HPO reference pressure is $0 \mathrm{~Pa}, \dot{m}_{L P O}=5.03 \mathrm{~kg} / \mathrm{s}$.

ISE and just before the pick-up tube, is relatively small. However, the decrease is total pressure over this part of the pipe is about 0.37 bar, so about half the kinetic energy is dissipated in the main part of the separator. This evolution of $\left\langle\bar{p}_{t o t}\right\rangle$ with $z$ is presented in figure 4.18, showing an exponential-like decay. A good fit is obtained for $z>0.40 \mathrm{~m}$ by $\left\langle\bar{p}_{t o t}\right\rangle=0.64 e^{-0.53 z}$. Therefore, of the 1.8 bar static pressure drop over the ISE, $60 \%$ is lost during the swirl generation and an additional $20 \%$ is lost before the flow reaches the pick-up tube. 


\section{Axial velocity $(\mathrm{m} / \mathrm{s})$}

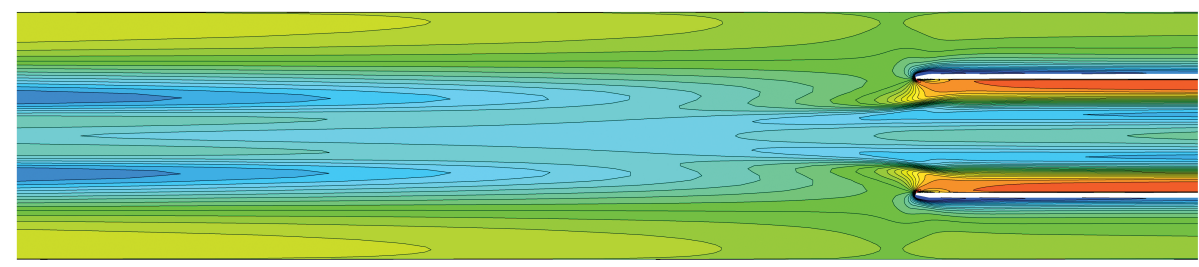

Figure 4.19: Time-averaged axial velocity near the pick-up tube on the plane $x=0 \mathrm{~m}$ through the separator. Inlet bulk velocity is $2 \mathrm{~m} / \mathrm{s}, \mathrm{HPO}$ reference pressure is $0 \mathrm{~Pa}, \dot{m}_{L P O}=5.03 \mathrm{~kg} / \mathrm{s}$.

\subsection{Flow near pick-up tube}

The pick-up tube has a significant influence on the flow. A contour plot of the timeaveraged axial velocity is given in figure 4.19, see also figure 4.10. A local velocity maximum seems to develop within the central minimum in downstream direction. The annular region of low axial velocity can be seen to widen as it approaches to pick-up tube. At the inner side of the pick-up tube, the axial velocity increases significantly.

On the outer surface of the pick-up tube, an adverse pressure gradient is experienced by the flow. This pressure gradient is stronger closer to the surface. The flow is decelerated in the region surrounding the outer surface and the flow reverses direction while it moves inward. The reversed flow is accelerated towards the leading edge of the pick-up tube, where the fluid flow bends around the leading edge, enters the pick-up tube and subsequently flows towards the LPO. The flow reversal is caused by the swirling flow and not by the suction effect due to a lower pressure inside the pick-up tube. When the LPO is closed this reversed flow region is still present. However, the size of the region of reversed flow surrounding the pick-up tube increases with increasing flow rate through the LPO.

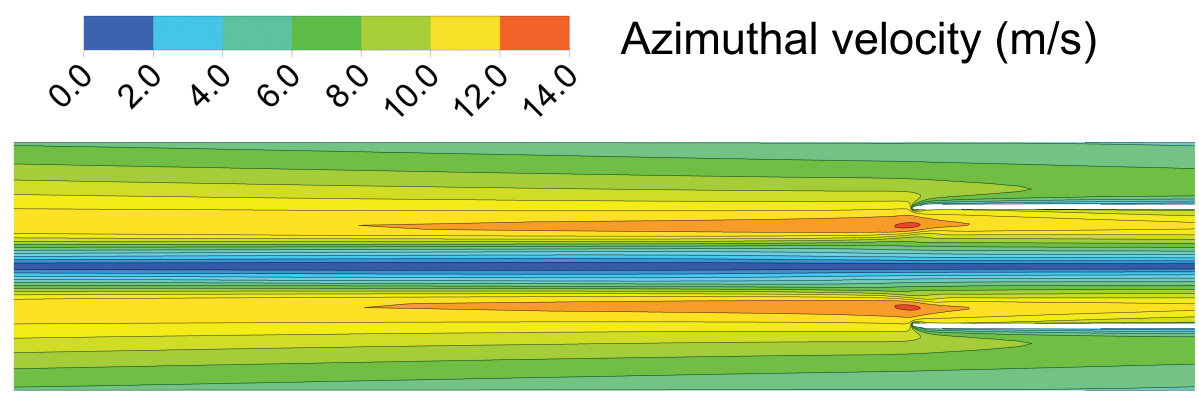

Figure 4.20: Time-averaged azimuthal velocity near the pick-up tube on the plane $x=0 \mathrm{~m}$ through the separator. Inlet bulk velocity is $2 \mathrm{~m} / \mathrm{s}, \mathrm{HPO}$ reference pressure is $0 \mathrm{~Pa}, \dot{m}_{L P O}=$ $5.03 \mathrm{~kg} / \mathrm{s}$. 
Although wall-functions are employed for the current results, a similar flow reversal was seen in previous simulations in which integration to the wall was carried out. The flow reversal is therefore not an artifact of the wall-functions.

The time-averaged azimuthal velocity field is depicted in figure 4.20, see also figures 4.11 and 4.12. As the flow enters the pick-up tube the azimuthal velocity increases due to vortex stretching. Also, a significant reduction in azimuthal velocity can be seen near the surface of the pick-up tube in the region between the pick-up tube and the pipe wall.

More insight into the flow can be gained by examining the two-dimensional streamlines on the plane through the separator, see figure 4.21. These streamlines have been constructed using the time-averaged two-dimensional velocity field on that plane. Since the timeaveraged flow field is axisymmetric, a three-dimensional streamline, which crosses the twodimensional streamline, keeps intersecting that two-dimensional streamline downstream. The two-dimensional streamlines are the intersections of the three-dimensional streamlines with the two-dimensional plane. Therefore, the two-dimensional streamlines give useful information on the three-dimensional flow field. The figure is divided into two parts by the white line at $r=0 \mathrm{~m}$. The background coloring on the top half is the axial velocity and on the lower half the azimuthal velocity. The streamlines are converging to the inner side of the surface of the pick-up tube and at the center. Here the axial and azimuthal velocities increase. Likewise, diverging streamlines are seen where the annular region with reversed flow inside the pick-up tube develops.

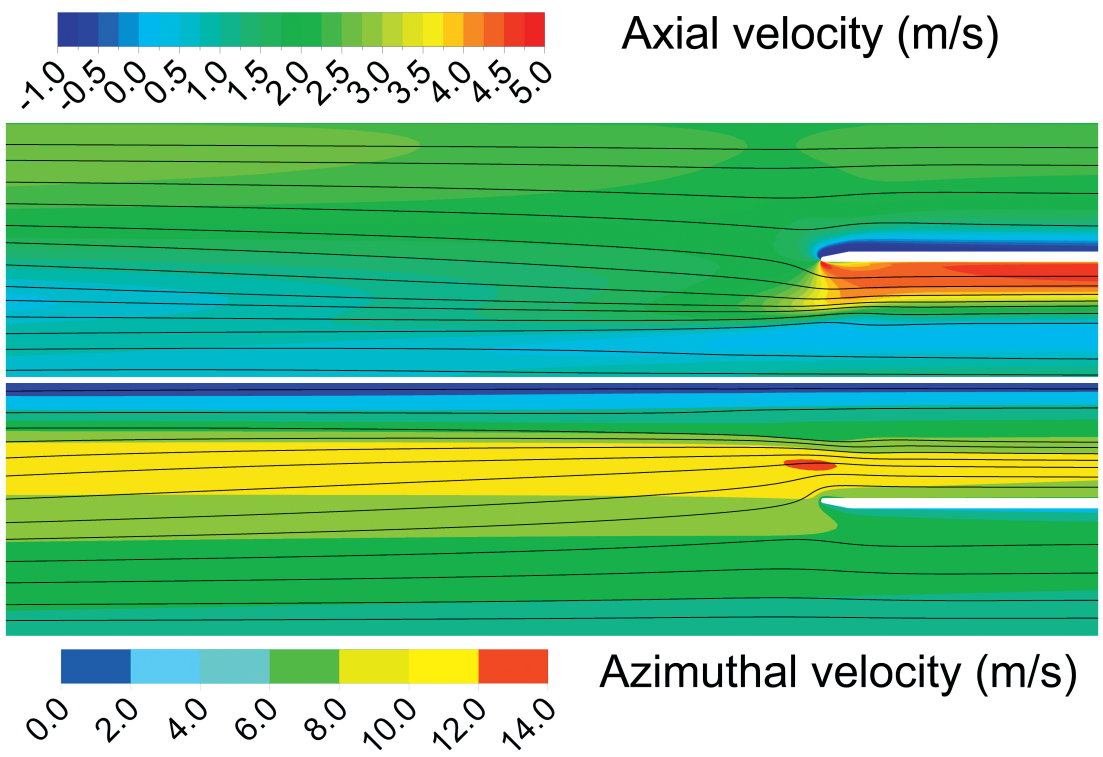

Figure 4.21: Two-dimensional streamlines of time-averaged velocity field on plane through separator axis near pick-up tube. Background coloring shows time-averaged axial (top half) and time-averaged azimuthal (bottom half) velocity divided by white line at $r=0 \mathrm{~m}$. Inlet bulk velocity is $2 \mathrm{~m} / \mathrm{s}, \mathrm{HPO}$ reference pressure is $0 \mathrm{~Pa}, \dot{m}_{L P O}=5.03 \mathrm{~kg} / \mathrm{s}$. 


\subsection{Parameter studies}

Sofar only the design point condition of the separator has been considered. In this section flow parameters are varied in order to obtain a better understanding of the behavior of the separator for other conditions than the design point condition.

\subsubsection{Dimension analysis}

The Buckingham $\Pi$ theorem is applied to find non-dimensional groups of variables to describe the flow. Restating the solution is terms of non-dimensional variables reduces the total number of variables that is to be varied in the parameter study. Furthermore, the use of non-dimensional variables can reveal patterns in the results otherwise obscured when dimensionfull variables are used. Examples of these patterns are self-similarity solutions, in which different solutions collapse into a single curve when expressed in terms of dimensionless variables. For self-similarity solutions the influence of one aspect of the flow, such as the Reynolds number, on the solution can often be clearly identified.

The Buckingham $\Pi$ theorems states that a results of a physical problem depending on $\beta$ variables can be rewritten into a dimensionless quantity depending on $\Pi$ non-dimensional variables. The number $\Pi$ is equal to $\beta$ minus the number of independent dimensions present in set $\beta$. For more information on the Buckingham $\Pi$ theorem the reader is referred to textbooks, for instance Fox and McDonald [38]. For the present case $\beta$ is seven. The seven variables are the inlet velocity $u_{z, b}$, the density $\rho$, the viscosity $\mu$, the flow rate through the LPO $Q_{L P O}$, flow deflection at the vanes, the pipe diameter $D$ and the distance between ISE and pick-up tube $L$.

The azimuthal velocity at the trailing edge of the vanes $u_{\theta, t e}$ is used as flow deflection scale. It is area-averaged on a cross-sectional plane just after the vanes. The variable characterizing the swirling flow is not unique. Here $u_{\theta, t e}$ is chosen since it can be unambiguously defined and is closely linked to the design of the ISE.

There are three independent dimensions: mass, length and time. The density $\rho$, velocity $u_{z, b}$ and pipe diameter $D$ are selected as recurring variables. Four non-dimensional numbers can be found by combining these three recurring variables with one of the four remaining variables from the set. The non-dimensional numbers describing the singlephase flow are the length ratio L', the flow split FS, the Reynolds number $\operatorname{Re}_{\mathrm{D}}$ and the trailing edge swirl angle Sw.

$$
\begin{aligned}
\mathrm{L}^{\prime} & =\frac{L}{D} \\
\mathrm{FS} & =\frac{Q_{L P O}}{\pi \rho u_{z, b} D^{2} / 4} \\
\mathrm{Re}_{\mathrm{D}} & =\frac{\rho u_{z, b} D}{\mu} \\
\mathrm{Sw} & =\frac{u_{\theta, t e}}{u_{z, b}}
\end{aligned}
$$


In subsequent chapters the length ratio $L / D=17$ at all times. The dimensionless numbers $\mathrm{FS}, \mathrm{Re}_{\mathrm{D}}$ and $\mathrm{Sw}$ will be varied in subsequent sections.

\subsubsection{Variation of flow rate}

Of interest is the change in the behavior of the flow in the separator with variation in flow rate $Q_{\text {inlet }}$. A change in flow rate amounts to a change in $\operatorname{Re}_{\mathrm{D}}$. Here the results of water flows with 75,100 and $125 \%$ of the design flow rate are discussed. This is an appropriate range for the flow rate, but leads only to a relatively small change in $\operatorname{Re}_{\mathrm{D}}$. To explore the effect of large variations in $\mathrm{Re}_{\mathrm{D}}$ also a numerical simulation for a single-phase oil flow is carried out. The properties of the oil are that of Vitrea 10. All relevant material properties, flow conditions and non-dimensional variables are listed in table 4.2. To mimic the flow in the experimental setup as closely as possible the measured material properties of water with $9 \mathrm{wt} \%$ salt are used.

The time-averaged non-dimensional axial and azimuthal velocity distributions in the crossflow plane at $z=0.50 \mathrm{~m}$ are shown in figures 4.22 and 4.23 , respectively. The distributions at $z=1.50 \mathrm{~m}$ are shown in figures 4.24 and 4.25 . The results for the single-phase water flows almost collapse onto a single curve, indicating that for this range of $\operatorname{Re}_{\mathrm{D}}$ the variation due to Reynolds number is insignificant. For dilute oil-water flows independency of $R_{D}$ can therefore be expected. However, more viscous fluids will, for typical flow rates, have a range of $\mathrm{Re}_{\mathrm{D}}$ for which viscous effects have a clear influence on the flow pattern.

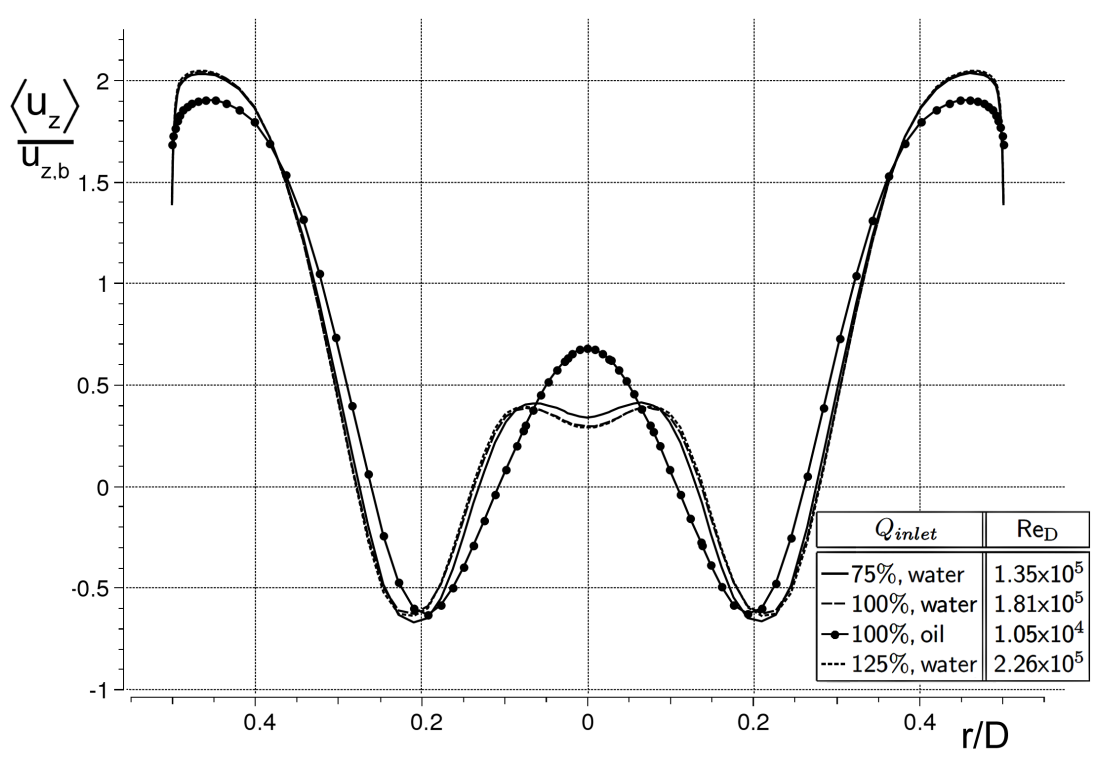

Figure 4.22: Radial distribution of the time-averaged non-dimensional axial velocity on a line through the center of the pipe at $z=0.50 \mathrm{~m}$ for variations in $\mathrm{Re}_{\mathrm{D}}$. Flow parameters are specified in table 4.2. 


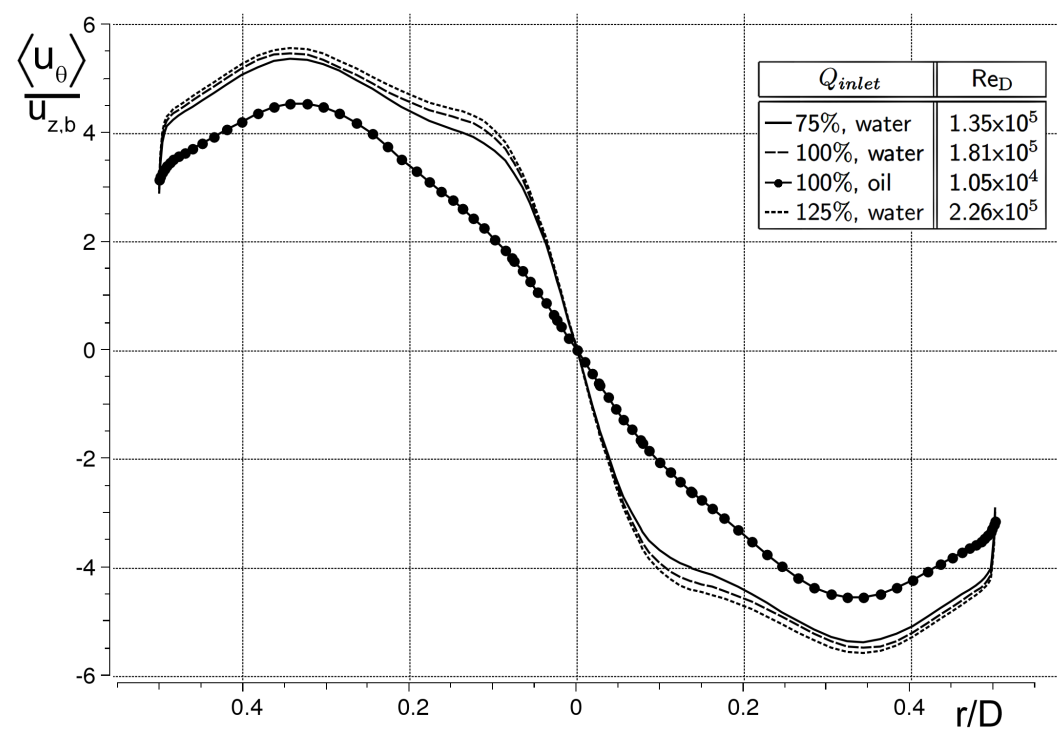

Figure 4.23: Radial distribution of the time-averaged non-dimensional azimuthal velocity on a line through the center of the pipe at $z=0.50 \mathrm{~m}$ for variations in $\mathrm{Re}_{\mathrm{D}}$. Flow parameters are specified in table 4.2 .

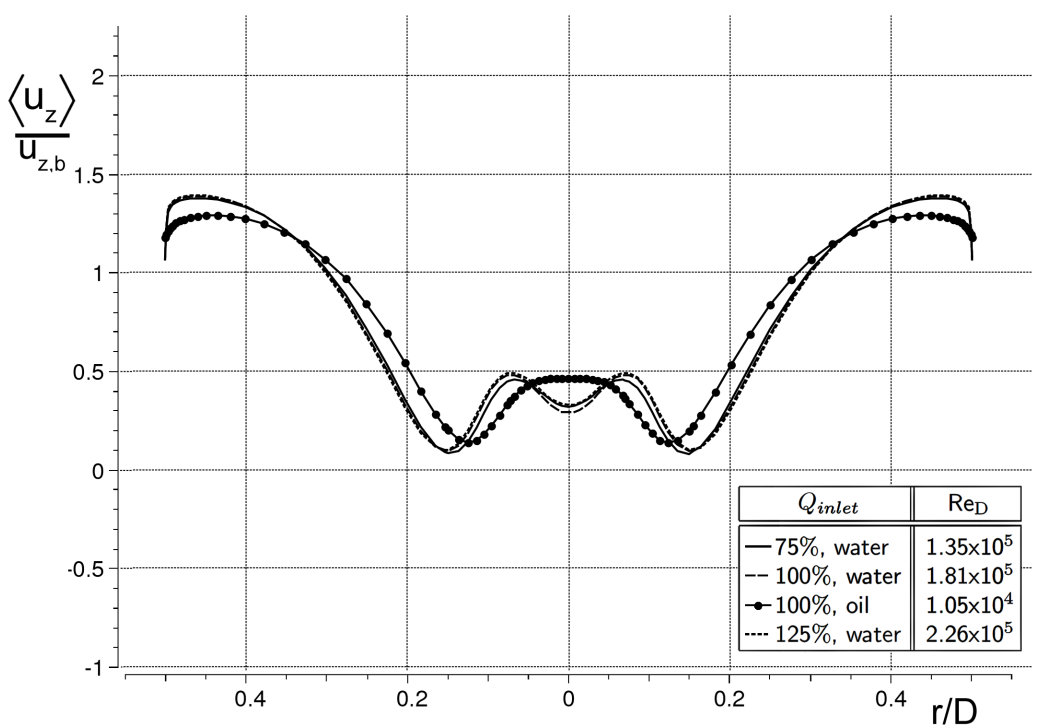

Figure 4.24: Radial distribution of the time-averaged non-dimensional axial velocity on a line through the center of the pipe at $z=1.50 \mathrm{~m}$ for variations in $\mathrm{Re}_{\mathrm{D}}$. Flow parameters are specified in table 4.2 . 


\begin{tabular}{|l|c|c|c||c|c|c||c|}
\hline \multicolumn{1}{|c|}{$Q_{\text {inlet }}$} & $\begin{array}{c}\rho \\
\left(\mathrm{kg} / \mathrm{m}^{3}\right)\end{array}$ & $\begin{array}{c}\mu \\
(\mathrm{kg} /(\mathrm{m} . \mathrm{s}))\end{array}$ & $\begin{array}{c}u_{z, b} \\
(\mathrm{~m} / \mathrm{s})\end{array}$ & $\mathrm{FS}$ & $\operatorname{Re}_{\mathrm{D}}$ & $\mathrm{Sw}$ & $\mathrm{Eu}$ \\
\hline \hline $75 \%$, water & 1067.8 & $1.183 \times 10^{-3}$ & 1.50 & 0.30 & $1.35 \times 10^{5}$ & 6.71 & 115.4 \\
$100 \%$, water & 1067.8 & $1.183 \times 10^{-3}$ & 2.00 & 0.30 & $1.81 \times 10^{5}$ & 6.72 & 118.2 \\
$100 \%$, oil & 869 & $16.5 \times 10^{-3}$ & 2.00 & 0.30 & $1.05 \times 10^{4}$ & 6.58 & 97.7 \\
$125 \%$, water & 1067.8 & $1.183 \times 10^{-3}$ & 2.50 & 0.30 & $2.26 \times 10^{5}$ & 6.73 & 120.6 \\
\hline
\end{tabular}

Table 4.2: Material properties, flow conditions and dimensionless variables in study on variation in the inlet flow rate.

The results of variation of $\operatorname{Re}_{\mathrm{D}}$ can no longer be deduced from the non-dimensional solution. Although the basic flow pattern, such as the $\mathrm{W}$-shape remains intact for flows at low $\operatorname{Re}_{\mathrm{D}}$, the distribution now does not show a reduction of the axial velocity at the center.

The distribution of the azimuthal velocity for the different $\mathrm{Re}_{\mathrm{D}}$ shows larger differences, also for $\operatorname{Re}_{\mathrm{D}}$ around the one of the design point. For the case of single-phase oil flow the reduction in azimuthal velocity is especially clear. A lower azimuthal velocity for low $\mathrm{Re}_{\mathrm{D}}$ flow will lead to lower centrifugal forces decreasing the separation efficiency. In addition, the dent in the distribution seen at $r / D \approx 0.1$ for $z=0.50 \mathrm{~m}$ is almost

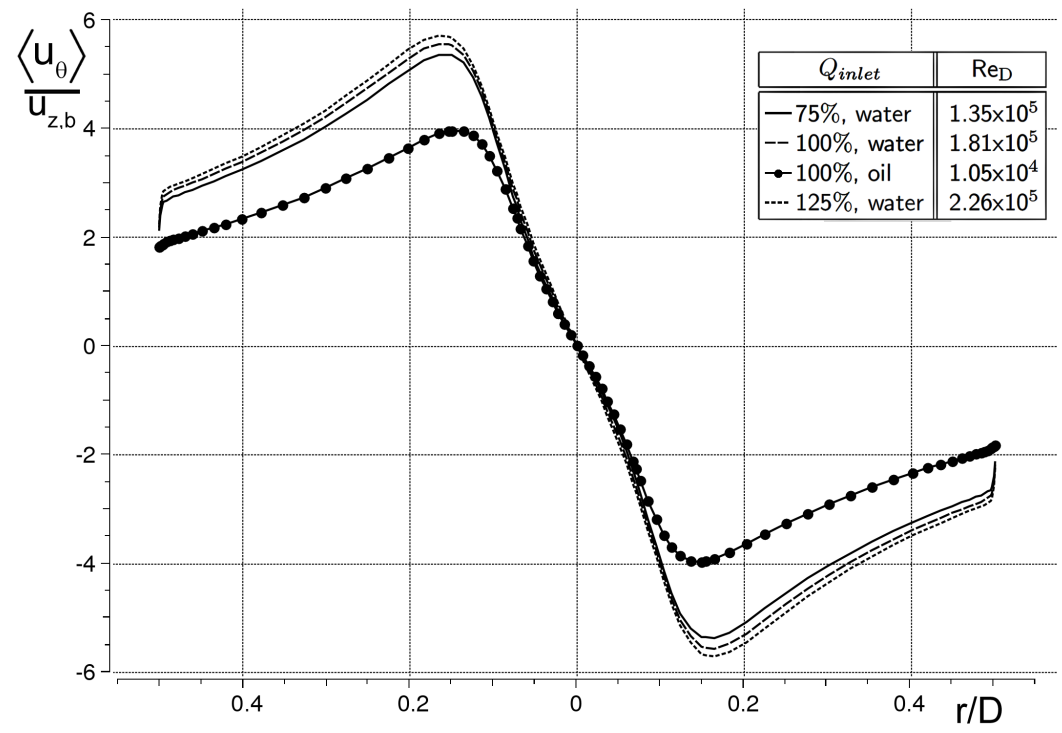

Figure 4.25: Radial distribution of the time-averaged non-dimensional azimuthal velocity on a line through the center of the pipe at $z=1.50 \mathrm{~m}$ for variations in $\mathrm{Re}_{\mathrm{D}}$. Flow parameters are specified in table 4.2 . 
smoothed out for the pure oil case, which also shows a lower value of the axial velocity here. Further downstream, at $z=1.50 \mathrm{~m}$, the differences in the distribution of the axial velocity resemble those for $z=0.50 \mathrm{~m}$. The distribution of the azimuthal velocity has now about the same slope in the center of the pipe for all $\operatorname{Re}_{\mathrm{D}}$ considered, but for the the low $\operatorname{Re}_{\mathrm{D}}$ it does not reach the levels it reaches for the higher $\operatorname{Re}_{\mathrm{D}}$.

The non-dimensional pressure on a line through the center of the pipe at $z=1.00 \mathrm{~m}$ is shown in figure 4.26. While the results for the azimuthal velocity show minor differences, the pressure distribution for the single-phase water cases clearly do not collapse onto a single curve, that is, there is a dependence on $\mathrm{Re}_{\mathrm{D}}$. The pressure difference between wall and the center of the pipe increases for increasing $R_{\mathrm{D}}$. The result for the single-phase oil flow clearly shows a much higher pressure in the center which corresponds to the much lower azimuthal velocity for this case, thus less solid-body rotation.

The area-averaged pressure difference $\Delta\langle\bar{p}\rangle$ between inlet and LPO is normalized by the dynamic pressure at the inlet to form the Euler number Eu

$$
\mathrm{Eu}=\frac{\Delta\langle\bar{p}\rangle}{\frac{1}{2} \rho u_{z, b}^{2}}
$$

Again, the pressure difference $\Delta\langle\bar{p}\rangle$ does not include the hydrostatic pressure. Figure 4.27 shows $\mathrm{Eu}$ as function of $\mathrm{Re}_{\mathrm{D}}$. The values of Eu are included in table 4.2. Only the single-phase water flow results are presented here since the pressure drop is sensitive to relatively small changes in the swirl angle Sw. Therefore, the results for single-phase oil flow does not lie in the $\operatorname{Re}_{\mathrm{D}}-\mathrm{FS}$ plane at constant $\mathrm{Sw}_{w}$ shown in figure 4.27. The curve is

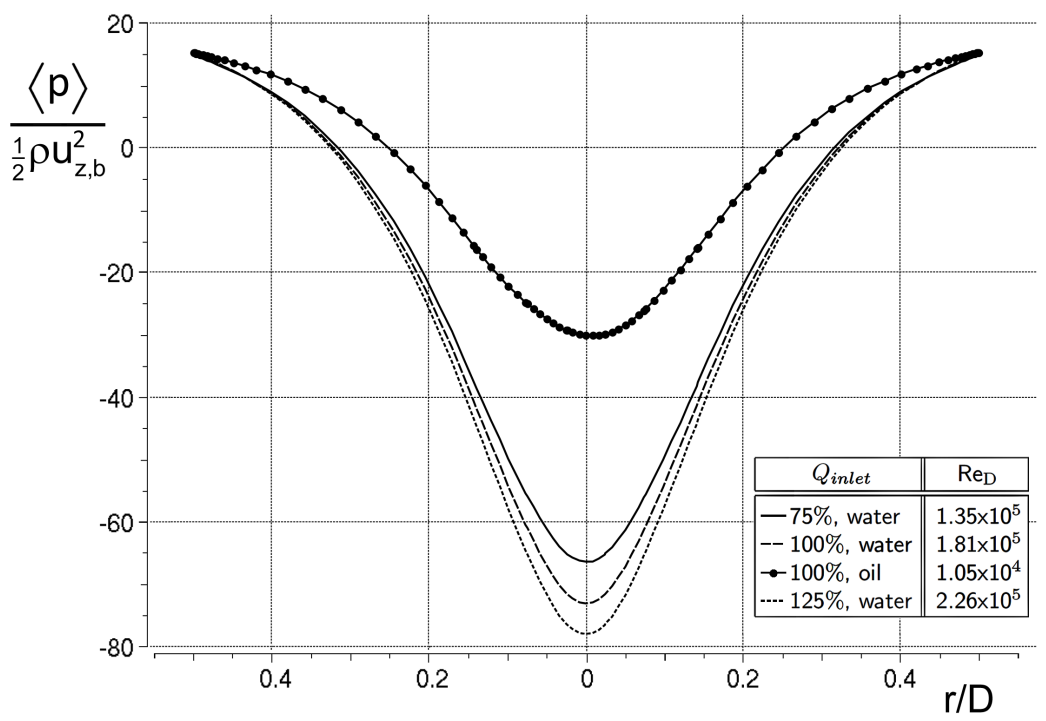

Figure 4.26: Radial distribution of the time-averaged non-dimensional pressure at $z=1.00 \mathrm{~m}$ for different values of $\mathrm{Re}_{\mathrm{D}}$. Flow parameters are specified in table 4.2. 
fitted to the data by a power law, which is given by

$$
\mathrm{Eu}=42.2 \operatorname{Re}_{\mathrm{D}} 0.085
$$

A higher pressure drop between inlet and LPO is expected for higher inlet velocities in the separator. However, here an increase in $\mathrm{Eu}$ is also observed for increasing $\mathrm{Re}_{\mathrm{D}}$. So the pressure drop scales as $\Delta\langle\bar{p}\rangle \propto u_{z, b}^{2.085}$.

The value of Eu in non-swirling pipe flow is given by

$$
\frac{\Delta p}{\frac{1}{2} \rho u_{z, b}^{2}}=f \frac{L}{D}
$$

Here $f$ is the friction factor which dependent on $\operatorname{Re}_{\mathrm{D}}$ and $L / D=17$. For turbulent pipe flow the friction factor $f$ always decreases with increasing $\operatorname{Re}_{\mathrm{D}}$. Contrary to non-swirling pipe flow, the non-dimensional pressure drop between inlet and LPO for strongly swirling flow increases with higher $\operatorname{Re}_{\mathrm{D}}$.

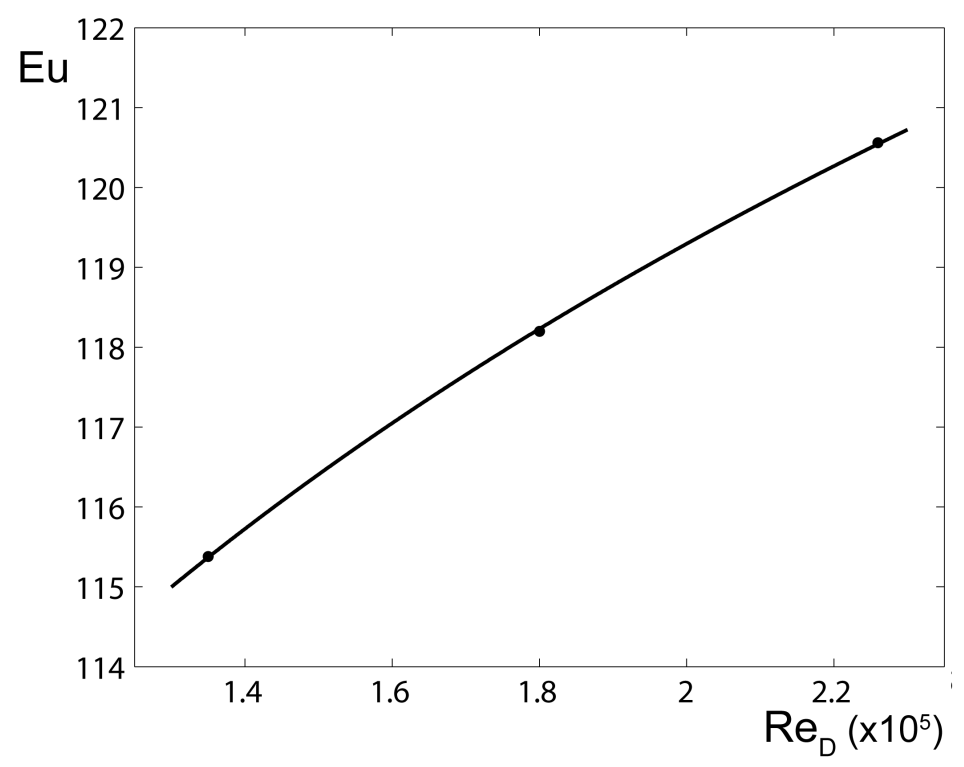

Figure 4.27: Dimensionless pressure drop from inlet to $L P O$ as function of $\mathrm{Re}_{\mathrm{D}}$ with power law curve fit for single-phase water flow. Data from table 4.2 is used. 


\subsubsection{Variation of flow split}

Another important flow parameter is the flow split FS. The separator is designed to handle mixtures in which water is the continuous phase, with a typical oil cut in the feed of around 15 to $40 \%$. The flow split is usually higher than the inlet oil volume fraction since cleaner water is in general more valued than dryer oil. Of interest is the performance of the separator for different feeds and therefore different flow splits. The variation of the flow split FS for the present study is FS $=[0.20,0.30,0.40,0.50]$.

The radial distributions of the time-averaged axial and azimuthal velocity on a line through the center of the pipe at $z=0.50 \mathrm{~m}$ are shown in figures 4.28 and 4.29 , respectively. A change in flow split alters the axial velocity distribution in a region more than 10 diameters upstream from the pick-up tube. With increasing flow split the central dip flattens and develops into an local maximum. However, the rest of the distribution is not much affected by the change in FS, it is only the central part, the core region that changes.

Larger differences, as well as over a larger region can be observed in the radial distribution of the azimuthal velocity. For increasing flow split the dent in the distribution at $r / D \approx 0.1$ is more pronounced and moves slightly inwards together with the increase in the strength of the solid-body rotation. Striking is this increase in magnitude of the azimuthal velocity for higher flow splits that far upstream of the pick-up tube.

The axial velocity distribution on a line through the center of the pipe at $z=1.50 \mathrm{~m}$ can be seen in figure 4.30. The axial velocity increases for higher flow splits in the region 0 $<r / D<0.275$. However, the axial velocity increase is not evenly distributed as the flow

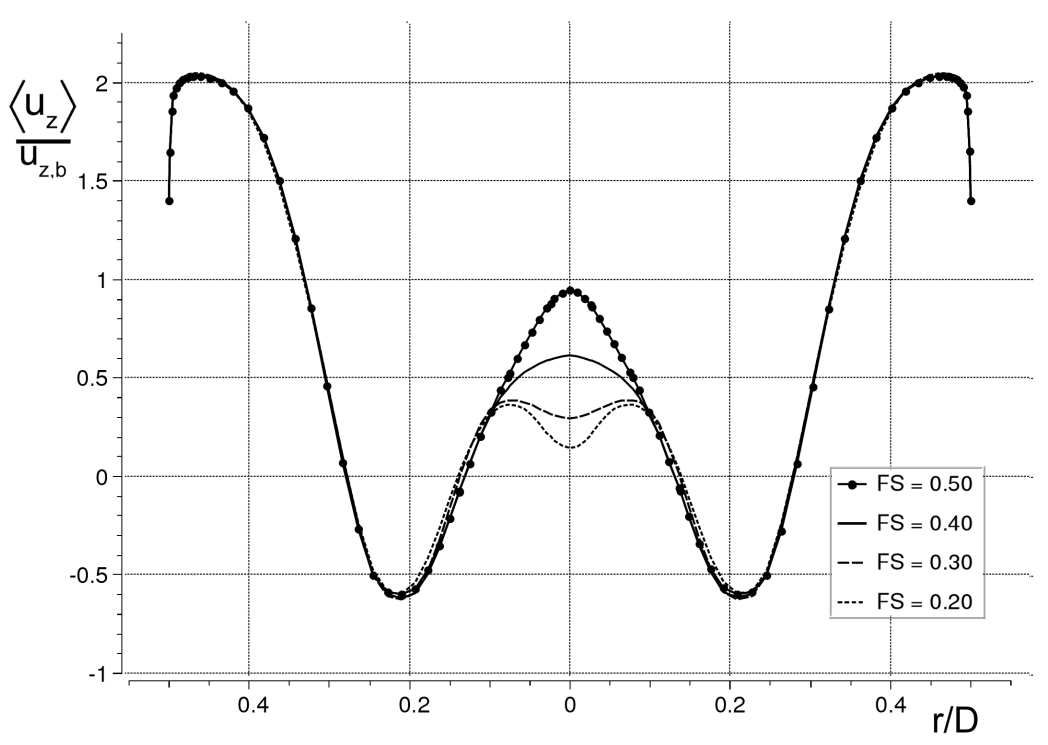

Figure 4.28: Radial distribution of the time-averaged non-dimensional axial velocity on a line through the center of the pipe at $z=0.50 \mathrm{~m}$ for flow split $\mathrm{FS}=[0.20,0.30,0.40,0.50]$. For all cases $\operatorname{Re}_{\mathrm{D}}=1.81 \times 10^{5}$ and $\mathrm{Sw}=6.72$. 


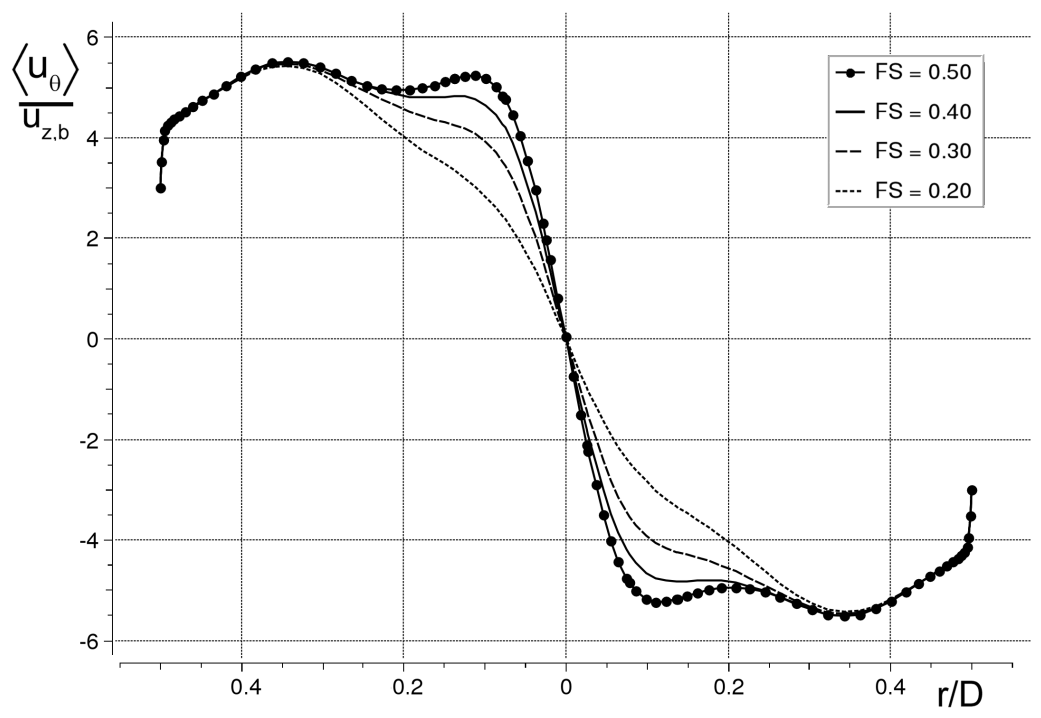

Figure 4.29: Radial distribution of the time-averaged non-dimensional azimuthal velocity on a line through the center of the pipe at $z=0.50 \mathrm{~m}$ for flow split FS $=[0.20,0.30,0.40,0.50]$. For all cases $\operatorname{Re}_{\mathrm{D}}=1.81 \times 10^{5}$ and $\mathrm{Sw}=6.72$.

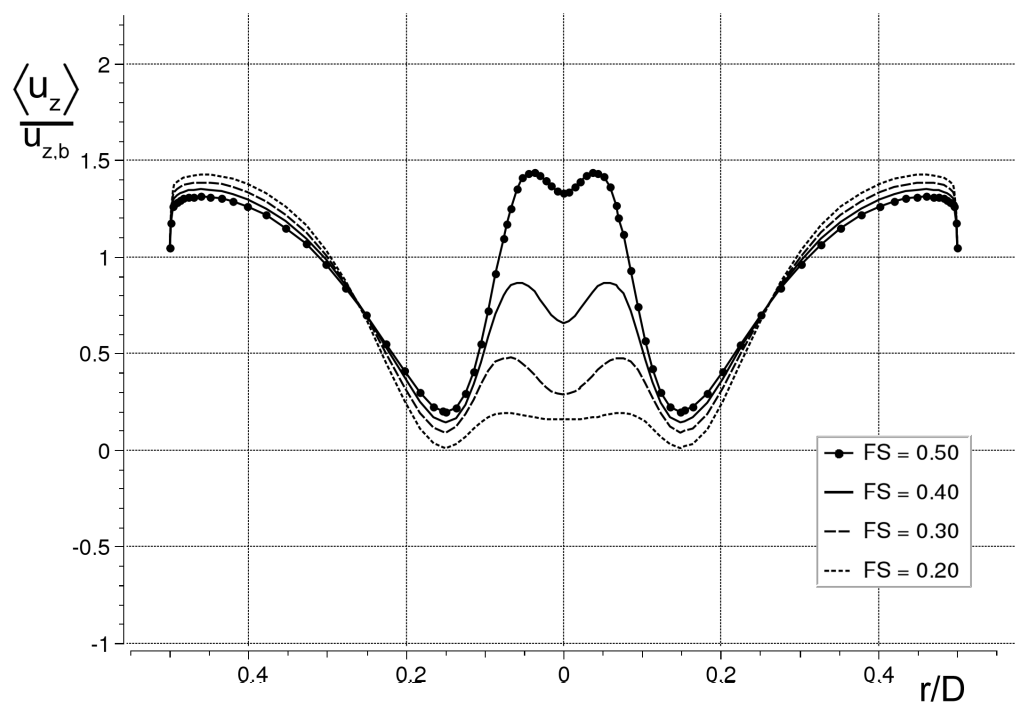

Figure 4.30: Radial distribution of the time-averaged non-dimensional axial velocity on a line through the center of the pipe at $z=1.50 \mathrm{~m}$ for flow split $\mathrm{FS}=[0.20,0.30,0.40,0.50]$. For all cases $\operatorname{Re}_{\mathrm{D}}=1.81 \times 10^{5}$ and $\mathrm{Sw}=6.72$. 
in the center is much more affected. In figure 4.31 the azimuthal velocity distribution on a line through the center of the pipe at $z=1.50 \mathrm{~m}$ is shown. Similar to the flow further upstream, in the core the swirl increases with increasing flow split and the inward motion of the location where the azimuthal velocity is maximal, is now more distinct. In addition, for flows with low flow split FS the solid-body rotation core has a lower slope in the center and the slope increases just outside the center. This forms a dent in the distribution of the azimuthal velocity, which is more pronounced for smaller FS and straightens out for increasing flow split.

While scaling the results with the inlet bulk velocity showed a weak dependence on $\operatorname{Re}_{\mathrm{D}}$, for the range considered, here no straightforward dependence of the flow on the flow split is found.

Due to the larger axial velocity in the center for higher flow splits more fluid will flow to the center. The inward motion of angular momentum leads to the observed higher azimuthal velocities and therefore to higher centrifugal forces acting on the dispersed phase in multiphase applications. The centrifugal acceleration on a line through the center of the pipe at $z=0.50 \mathrm{~m}$ and $1.50 \mathrm{~m}$ for FS varying from 0.20 to 0.50 is presented in figures 4.32 and 4.33 , respectively. As shown in the figures the maximum centrifugal force rises substantially for increasing flow split FS. The motion of the location of the maximum centrifugal force with respect to the pipe axis does not show a consistent trend. For a high flow split of 0.40 and 0.50 the distance between location of the maximum centrifugal

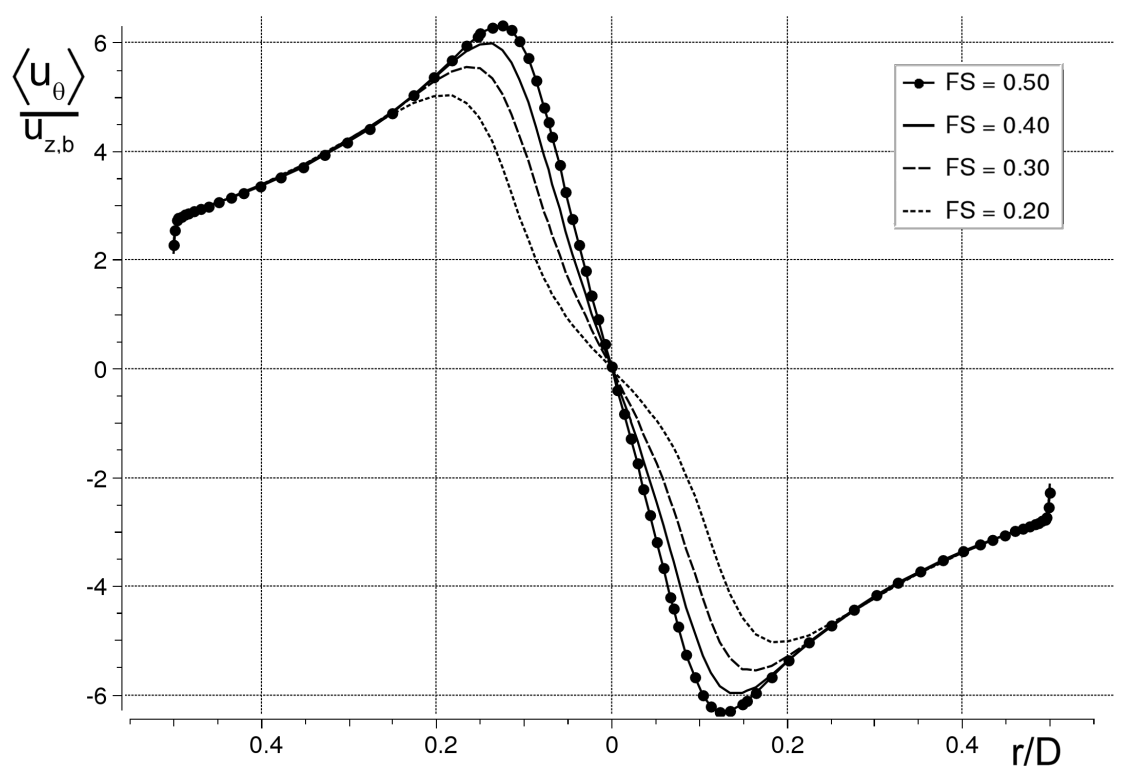

Figure 4.31: Radial distribution of the time-averaged non-dimensional azimuthal velocity on a line through the center of the pipe at $z=1.50 \mathrm{~m}$ for flow split FS $=[0.20,0.30,0.40,0.50]$. For all cases $\operatorname{Re}_{\mathrm{D}}=1.81 \times 10^{5}$ and $\mathrm{Sw}=6.72$. 


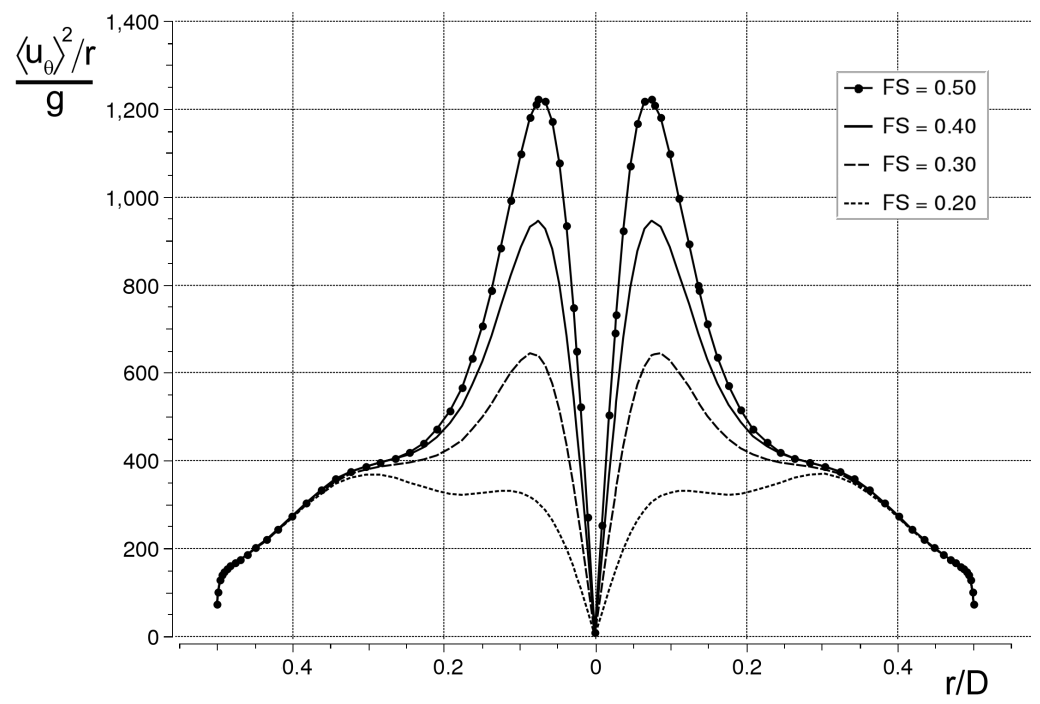

Figure 4.32: Radial distribution of the time-averaged non-dimensional centrifugal acceleration on a line through the center of the pipe at $z=0.50 \mathrm{~m}$ for flow split FS $=[0.20,0.30,0.40$, 0.50 ]. For all cases $\operatorname{Re}_{\mathrm{D}}=1.81 \times 10^{5}$ and $\mathrm{Sw}=6.72$.

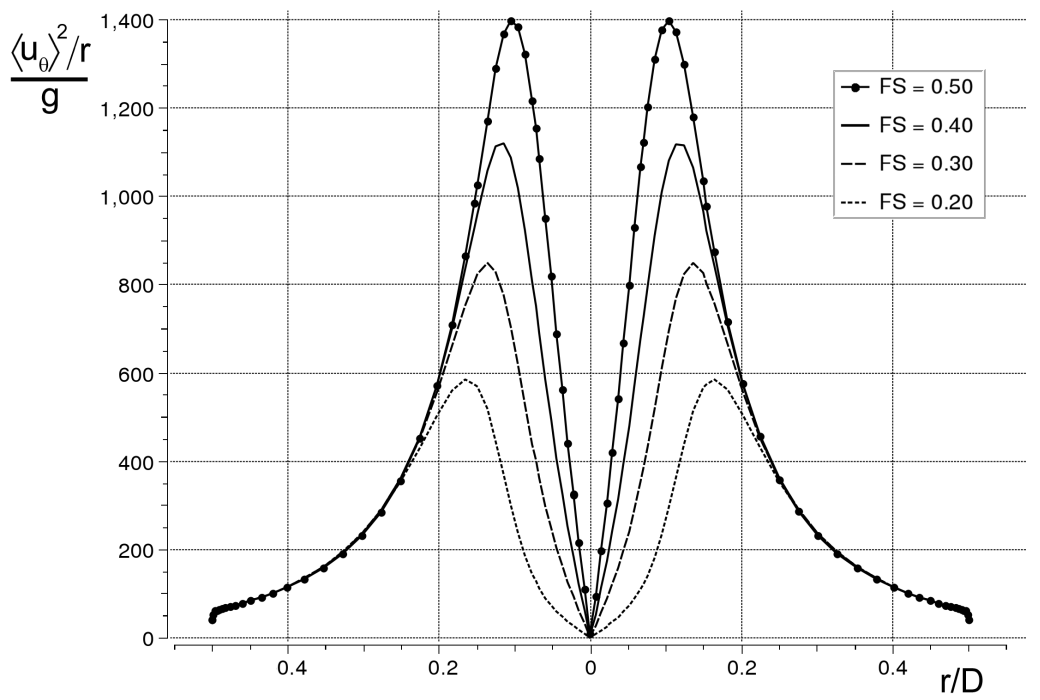

Figure 4.33: Radial distribution of the time-averaged non-dimensional centrifugal acceleration on a line through the center of the pipe at $z=1.50 \mathrm{~m}$ for flow split FS $=[0.20,0.30,0.40$, 0.50 ]. For all cases $\operatorname{Re}_{\mathrm{D}}=1.81 \times 10^{5}$ and $\mathrm{Sw}=6.72$. 


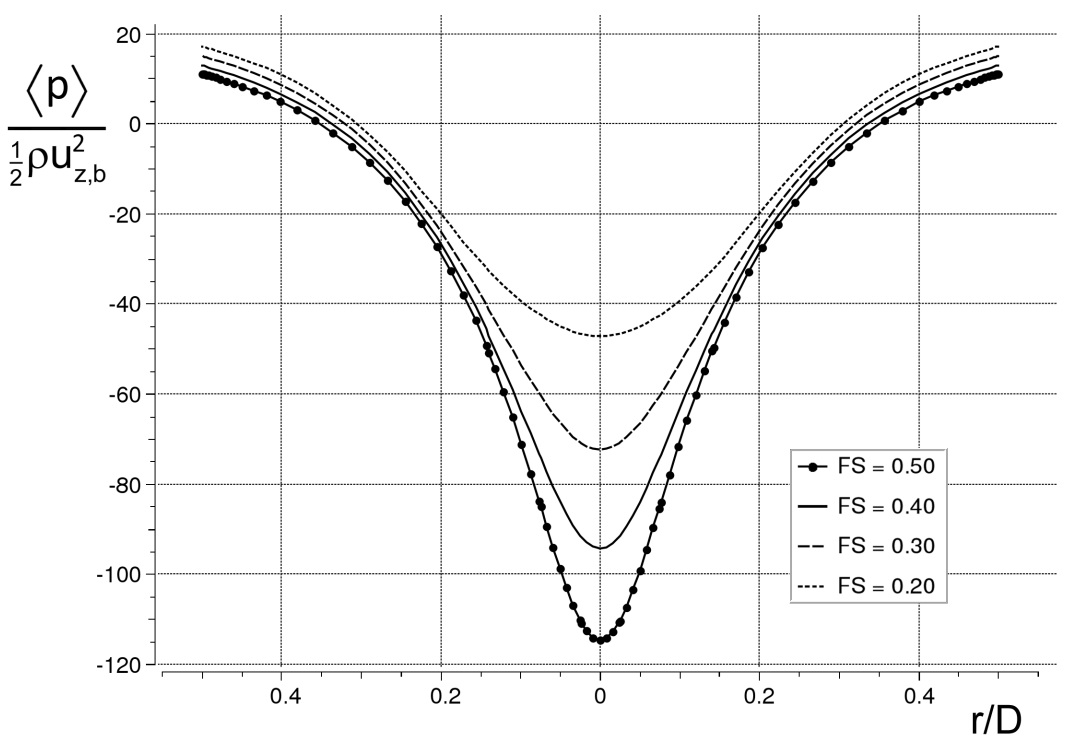

Figure 4.34: Radial distribution of the time-averaged non-dimensional pressure on a line through the center of the pipe at $z=1.00 \mathrm{~m}$ for flow split $\mathrm{FS}=[0.20,0.30,0.40,0.50]$. For all cases $\operatorname{Re}_{\mathrm{D}}=1.81 \times 10^{5}$ and $\mathrm{Sw}=6.72$.

force and the center of the pipe increases with increasing $z$, while for lower flow splits the location of the maximum centrifugal force initially moves radially outwards and starts to move inwards further downstream. However, at a fixed axial station the location of the maximum centrifugal force is closer to the axis of the pipe for increasing flow split.

Due to the strong variations observed in the g-forces acting on droplets, the separation efficiency for oil-water mixtures will increase for higher flow splits. Therefore it may be beneficial to design, for a given oil cut in the feed, a separator with a relatively small diameter pick-up tube and thus a higher axial velocity in the center of the pipe. This will lead to vortex stretching and therefore to a spin-up of the azimuthal velocity, achieving higher centrifugal forces. Although, for higher flow splits the residence times of the oil will be shorter due to the increased axial velocity in the center. Therefore, the oil phase will have less time to separate. Moreover, for increased flow split higher turbulent kinetic energy and dissipation rates are seen. These two effects reduce the gains in separation efficiency to some, probably small, extent.

However, at the same time the higher flow split will lead to a larger pressure drop over the separator. The dimensionless pressure on a line through the center of the pipe at $z=$ $1.00 \mathrm{~m}$ is shown in figure 4.34. The pressure difference between the wall and the center of the pipe doubles when the flow split is increased from 0.20 to 0.50 . In figure 4.35 the dimensionless pressure drop Eu is shown as a power law fit. The dimensionless pressure drop Eu as function the flow split FS is fitted as

$$
\mathrm{Eu}=145.5 \mathrm{FS}^{0.17}
$$




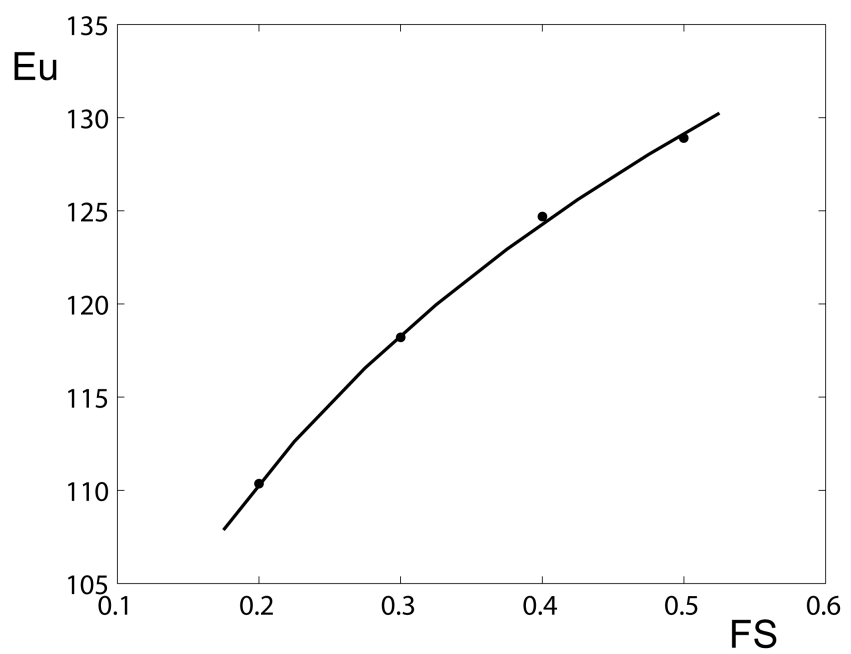

Figure 4.35: Euler numbers Eu as function of flow split with power law fit Eu $=145.5 \mathrm{FS}^{0.17}$. For all cases $\operatorname{Re}_{\mathrm{D}}=1.81 \times 10^{5}$ and $\mathrm{Sw}=6.72$.

So, as expected, an increase in pressure drop is seen for increasing flow split, although the increase in $\mathrm{Eu}$ is not strong. The increase in pressure drop decreases for higher flow split.

\subsubsection{Variation of swirl angle}

The shape of the vanes of the ISE has been determined by the design procedure discussed in chapter 3. An ISE with a different vane deflection angle will lead to a change in the downstream velocity distribution and will therefore alter the separation characteristics. Not only will a stronger deflection result in higher g-force on the dispersed phase, it will likely lead to more turbulence, higher pressure drop and possibly to droplet break-up. The increased turbulence level could remix the segregated phases, undoing the effect of the higher centrifugal forces.

An optimized design is desired for commercial application of this technology. However, a thorough study of design variations falls beyond the scope of the present study. Instead results are presented here for a few swirl angles to give an outline of its effect on the flow field.

A complete re-design of the ISE, essentially following the steps in chapter 3 once more, and subsequent meshing efforts would take an unwarranted amount of time. Therefore, a simplified geometry is used in which the nose section, the vanes and the initial part of the afterbody are removed. The velocity components are directly imposed on the newly formed inlet boundary. Figure 4.36 shows the original geometry and the simplified geometry.

The differences introduced by not resolving the vanes can be evaluated by comparing the 
solution for the vane-less configuration with that for the full configuration. Therefore, area-averaged velocity components for the full geometry have been determined at the location corresponding to the inlet of the vanes-less geometry. These area-averaged values $u_{z}^{v l}=5.35$ and $u_{\theta}^{v l}=13.44$ are subsequently used as input for the numerical simulation of the flow in the vanes-less configuration. It turns out that for the vane-less geometry the flow is more unsteady than for the original complete configuration. Nevertheless the results also show that the time-averaged velocity distributions of the two configurations agree very well, as will be shown later. However, for the vane-less geometry the Reynolds stresses and turbulent dissipation are considerably lower, since the upstream shear layers are absent. However, these effect do not influence the velocity distribution strongly. On the other hand it is expected that in the case of two-phase flow, the numerical simulations for polydispersed multiphase flow using a vane-less geometry will not yield accurate results for the variation in droplet size. This is primarily because much of the droplet break-up is expected to occur around the ISE, especially in the vane section.

Four simulations for the vane-less geometry have been carried out for the flow parameters given in table 4.3. Lower azimuthal velocities are chosen here because the safety margins used in the design, such as $\epsilon_{S W}$, make it likely that enough centrifugal force is available and lower velocities may still lead to the required separation but for a lower pressure drop. The distribution of the time-averaged axial velocity on the plane $x=0 \mathrm{~m}$ through the upstream part of the separator for the four cases given in table 4.3 and the full geometry case are shown in figure 4.37. The vanes in the full geometry cause non-axisymmetric features in the flow surrounding the afterbody. These features are not present in the results of the simulation for the vane-less configurations with $\mathrm{S}_{w}=6.72$. Therefore, differences can be seen in the darkly colored reversed flow region near the afterbody of the ISE. However, these features are smoothed out rapidly and the results for the vane-less geometry do not differ significantly from the results for the vaned geometry in the velocity distribution further downstream.

For the medium swirl case the flow directly aft of the afterbody shows that the detachment
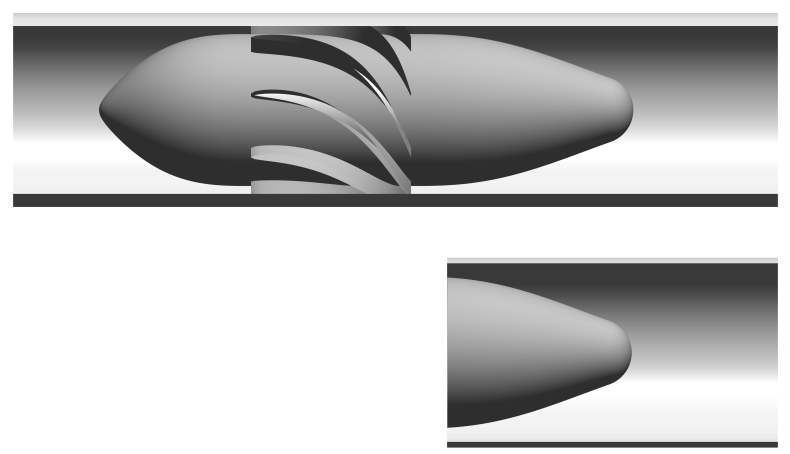

Figure 4.36: Cut-away view of full ISE geometry (top) and geometrically simpler vane-less geometry (bottom) used for the study of the effect of swirl angle variation. In vane-less geometry constant axial velocity $u_{z}^{v l}$ and azimuthal velocity $u_{\theta}^{v l}$ are imposed on inlet. 


\begin{tabular}{|l|c|c||c|c|c|}
\hline & $u_{z}^{v l}(\mathrm{~m} / \mathrm{s})$ & $u_{\theta}^{v l}(\mathrm{~m} / \mathrm{s})$ & $\mathrm{Sw}(-)$ & $\mathrm{FS}$ & $\mathrm{Re}_{\mathrm{D}}$ \\
\hline \hline nominal swirl & 5.35 & 13.44 & 6.72 & 0.30 & $1.81 \times 10^{5}$ \\
medium swirl & 5.35 & 5.00 & 2.50 & 0.30 & $1.81 \times 10^{5}$ \\
low swirl & 5.35 & 1.00 & 0.50 & 0.30 & $1.81 \times 10^{5}$ \\
zero swirl & 5.35 & 0.00 & 0.00 & 0.30 & $1.81 \times 10^{5}$ \\
\hline
\end{tabular}

Table 4.3: Inlet conditions for study of variation in swirl angle.

of the flow from the afterbody is delayed. Possibly criteria for flow detachment depending on the swirl strength are satisfied at a location further downstream when the swirl is reduced. The azimuthal velocity, which increases along the surface of the afterbody due to the conservation of angular momentum, needs a longer distance to increase such that these criteria are satisfied. Previous preliminary simulations also indicated that the detachment line moves upstream for stronger swirl.

Flow detachment and regions of reversed flow are also seen for the cases of low and zero swirl. The location of flow detachment is approximately the same for the latter two cases. So the downstream movement of the flow detachment for lower swirl is not continued here. Also, the shape of the reversed flow region for the cases of low and zero swirl is different compared to the one for the cases at the higher swirl. For the nominal and medium swirl angles the reversed flow region has, at its inception on the afterbody, a front which is almost perpendicular to the surface of the afterbody. This is not seen for the low and zero swirl cases, for which the angle of the reversed flow front with the surface
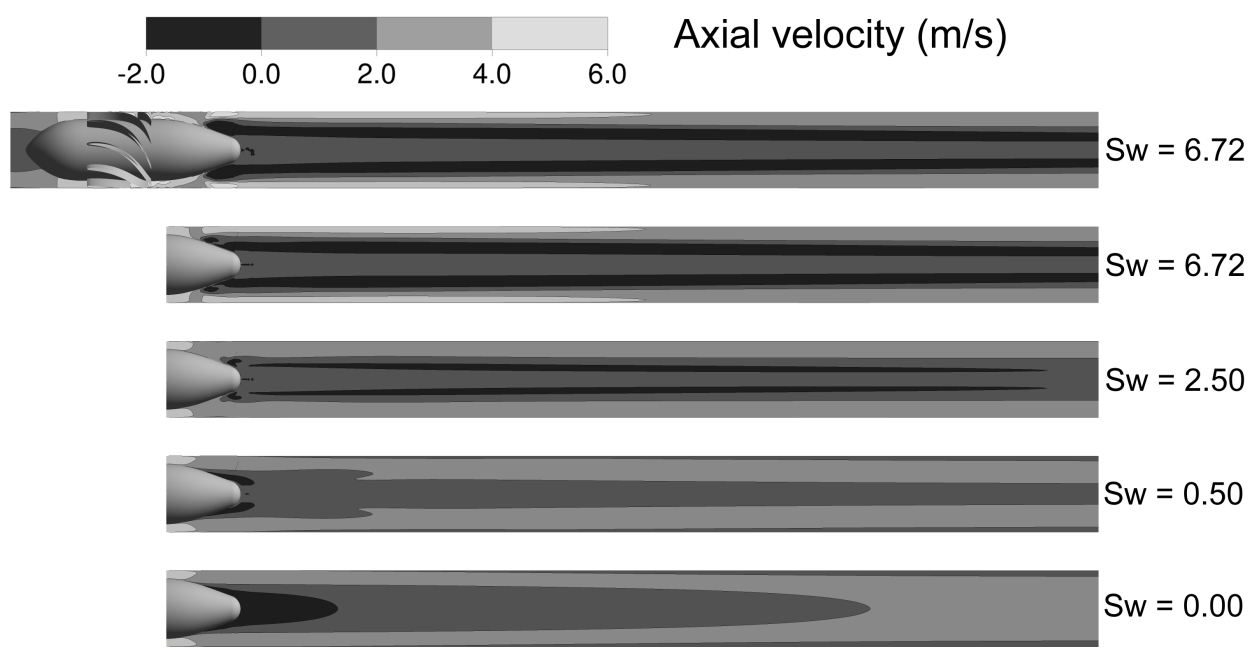

Figure 4.37: Contours of time-averaged axial velocity in the region downstream of the vanes of the ISE on a plane through of separator for variations swirl angel Sw. 
of the afterbody is much smaller.

Inspection of the axial pressure gradient shows that for the nominal and medium swirl cases, the increasing velocity near the afterbody yields a negative pressure gradient in the region upstream of the flow detachment. On the contrary, for the cases of low and zero swirl an adverse pressure gradient along the afterbody is seen. It is believed that for the latter two cases this flow separation is caused by the adverse pressure gradient instead of the swirl.

The time-averaged axial velocity distribution on a line through the center of the pipe at $z=0.50 \mathrm{~m}$ is shown in figure 4.38. Clear differences can be observed in the results for different swirl angles. By decreasing the swirl angle the difference between maximum and minimum velocity seen in the cross-plane also decreases. For medium swirl, the central dip in the radial distribution of the axial velocity is no longer present. Also the annular region of reversed flow becomes narrower, which may be explained by the smaller radius at which the flow detaches from the afterbody. The interaction of the swirl and specific afterbody geometry is not clear.

For low swirl the axial flow pattern changes from a W-shaped into a $\mathrm{V}$-shaped distribution. This V-shaped velocity distribution was previously observed, among others, by Kitoh [57] and Dirkzwager [29]. The case of zero swirl shows that the wake caused by the ISE is much wider and less pronounced than the central decrease in axial velocity in the case of low swirl. Moreover, the wake in the case of zero swirl smoothens out in the downstream direction and eventually a flat profile develops at $z=1.00 \mathrm{~m}$. The $\mathrm{V}$-shape distribution in the low swirl case is maintained in downstream direction, indicating that this flow pattern

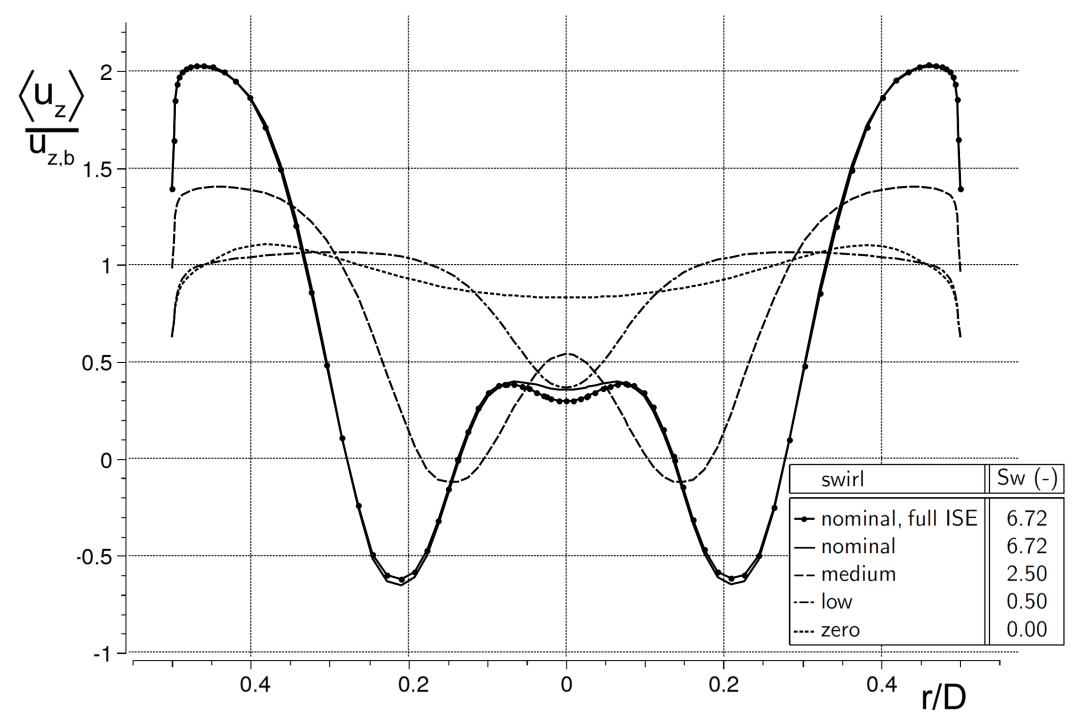

Figure 4.38: Radial distribution of the time-averaged non-dimensional axial velocity on a line through the center of the pipe at $z=0.50 \mathrm{~m}$ for the different swirl angles specified in table 4.3. For all cases $\operatorname{Re}_{\mathrm{D}}=1.80 \times 10^{5}$ and $\mathrm{FS}=0.3$. 
is primarily caused by the swirl and not by the wake of the ISE.

The radial distribution of the time-averaged azimuthal velocity on a line through the center of the pipe at $z=0.50 \mathrm{~m}$ is shown in figure 4.39. For the case of nominal swirl, $\mathrm{Sw}=6.72$, a dent in the azimuthal velocity at $r / D \approx 0.1 \mathrm{~m}$ is seen, followed by a region with a lower gradient until the maximum is reached. This change in the gradient of the azimuthal velocity is also seen in the case of medium swirl, for which $S w=2.50$, but here it is less distinct. However, the change in the gradient is absent in the case of low swirl, for which $\mathrm{Sw}=0.50$. For the case of low swirl a different flow pattern surrounds the afterbody, which is due to the different mechanism of flow detachment. For the case of low swirl, instead of an increase in azimuthal velocity near the surface of the afterbody, the azimuthal velocity is decreasing in the zone upstream of the flow separation. In addition, the location of the maximum azimuthal velocity is shifting towards the center for lower swirl.

Figures 4.40 and 4.41 show the distribution of the time-averaged axial and azimuthal velocity on a line through the center at $z=1.50 \mathrm{~m}$, respectively. The case of medium swirl now shows a local minimum in the center just as seen for the stronger swirling nominal case. Also the case of low swirl starts to form a local maximum in the center, which increases in the downstream direction. This gives the axial velocity distribution a $\mathrm{W}$-shape, just as for the case of medium swirl at $z=0.50 \mathrm{~m}$. For the case of low swirl the annular region of low velocity disappears inside the pick-up tube and a jet-like velocity profile is seen in the center. However, further downstream in the pick-up tube

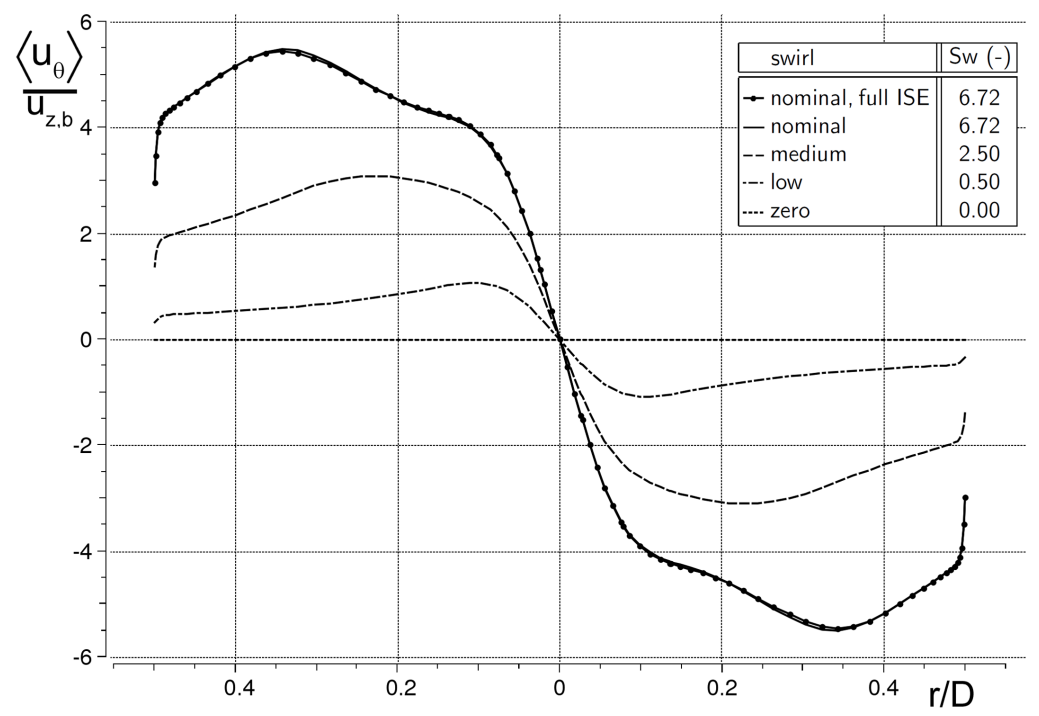

Figure 4.39: Radial distribution of the time-averaged non-dimensional azimuthal velocity on a line through the center of the pipe at $z=0.50 \mathrm{~m}$ for the different swirl angles specified in table 4.3. For all cases $\operatorname{Re}_{\mathrm{D}}=1.80 \times 10^{5}$ and $\mathrm{FS}=0.3$. 


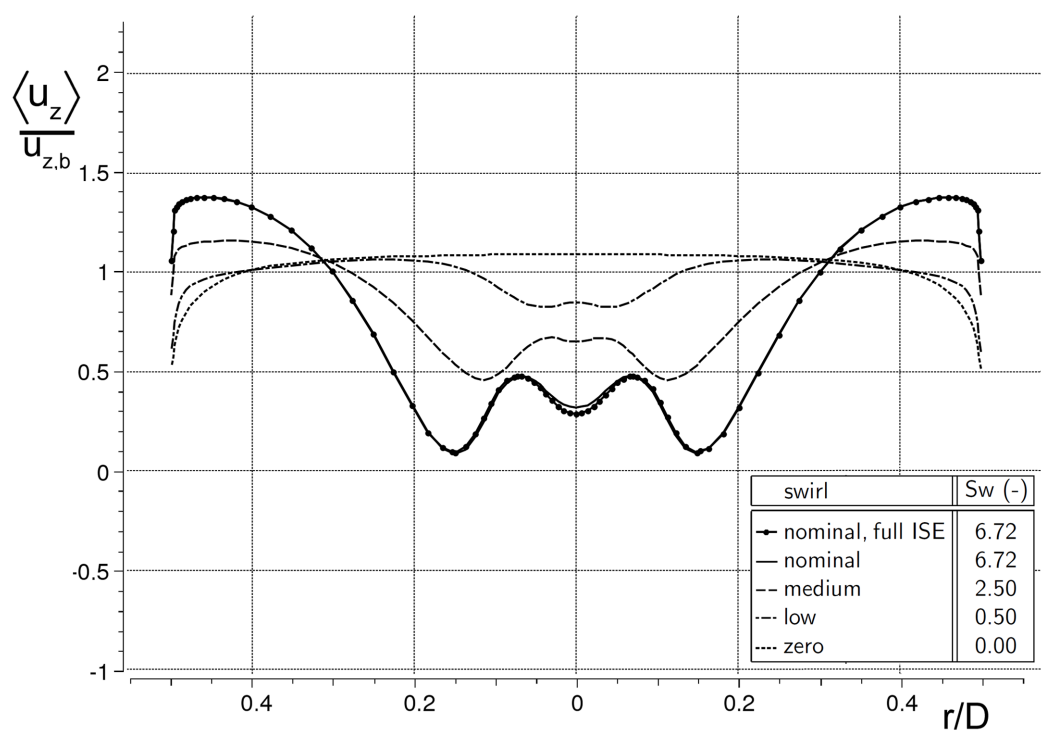

Figure 4.40: Radial distribution of the time-averaged non-dimensional axial velocity on a line through the center of the pipe at $z=1.50 \mathrm{~m}$ for the different swirl angles specified in table 4.3. For all cases $\operatorname{Re}_{\mathrm{D}}=1.80 \times 10^{5}$ and $\mathrm{FS}=0.3$.

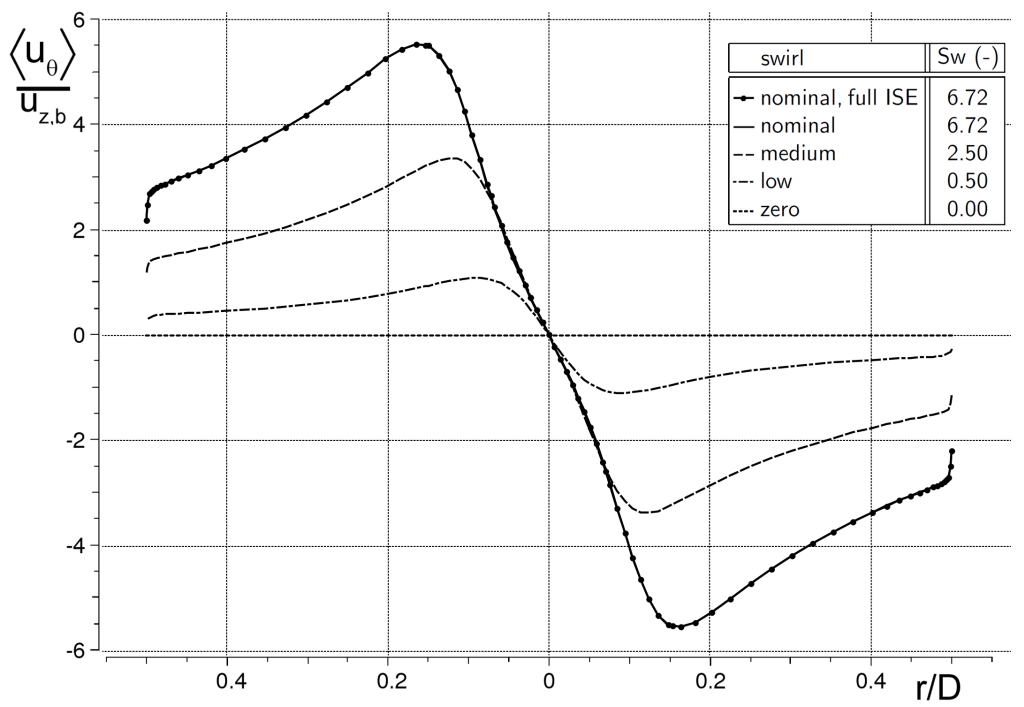

Figure 4.41: Radial distribution of the time-averaged non-dimensional azimuthal velocity on a line through the center of the pipe at $z=1.50 \mathrm{~m}$ for the different swirl angles specified in table 4.3. For all cases $\operatorname{Re}_{\mathrm{D}}=1.80 \times 10^{5}$ and $\mathrm{FS}=0.3$. 
the $\mathrm{V}$-shaped of the axial velocity distribution is recovered. As stated before the wake in the case of zero swirl has disappeared and a plug flow type of velocity distribution is seen. The distribution of the azimuthal velocity at $z=1.50 \mathrm{~m}$ shows similar features as observed at $z=0.50 \mathrm{~m}$, that is a lower maximum which moves inward with decreasing swirl angle. The azimuthal velocity distribution appears to collapse in the center, showing the same velocity gradient, that is, the same level of axial vorticity and solid-body rotation. This is not seen at upstream and downstream locations and is considered a coincidence. Contrary to the variation in $\mathrm{Re}_{\mathrm{D}}$, no straightforward trend has been found for results for variation in the swirl angle.

The radial distribution of the time-averaged non-dimensional pressure on a line through the center of the pipe at $z=1.00 \mathrm{~m}$ for the variations in swirl angle specified in table 4.3 is shown in figure 4.42. The increase in pressure difference between the pipe wall and the center with increasing swirl angle Sw can clearly be seen. Also the pressure at the pipe wall increases for increasing Sw. This is caused by the boundary condition at the HPO. For higher Sw the azimuthal velocity is higher in the annular channel towards the HPO. A higher incoming azimuthal velocity leads to a higher pressure drop over the flow straightener. Since the reference pressure is set to zero at the HPO, the pressure near the wall further upstream is higher for higher Sw.

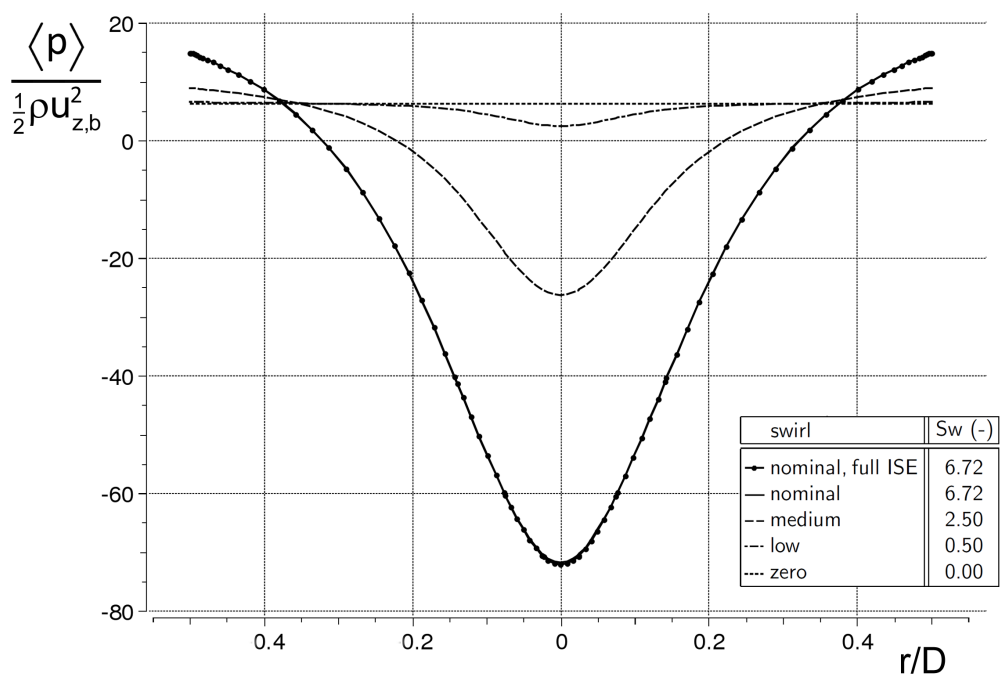

Figure 4.42: Radial distribution of the time-averaged non-dimensional pressure on a line through the center of the pipe at $z=1.00 \mathrm{~m}$ for the differnt swirl angles specified in table 4.3. For all cases $\operatorname{Re}_{\mathrm{D}}=1.80 \times 10^{5}$ and $\mathrm{FS}=0.3$. 


\subsection{Conclusions}

The analysis of the single-phase flow in the in-line separator shows a complicated flow pattern with a W-shaped radial distribution of the axial velocity. A straightforward, mechanistic explanation of this flow pattern is not available. The region of annular reversed flow is validated using experimental data from the TUD. Quantitative differences in the radial distribution of the time-averaged velocity components are found when comparing numerical and experimental results. However, the overall agreement is reasonable. Therefore, RANS CFD is deemed adequate to perform parameter studies to investigate the behavior of the flow in the separator and its performance in terms of separation efficiency.

In the separator the static pressure varies considerably. For the investigated design the swirling flow is generated at the expense of a 1.8 bar pressure drop over the ISE. The swirling flow leads to a parabolic-like radial distribution of the pressure, with a minimum pressure in the center. The maximum pressure difference between the pipe wall and center is 2.2 bar. The pressure differences between inlet and HPO and between inlet and LPO are 1.6 and 2.5 bar, respectively. Examination of the distribution of the total pressure indicates that it is unlikely that pressure recovery from de-swirling the flow is worthwhile. The velocity distributions scale linearly with the inlet bulk velocity when the flow rate is varied. The $\mathrm{W}$-shaped distribution of the axial velocity stays intact within a range of $\mathrm{Re}_{\mathrm{D}}$ of more than an order of magnitude. For low-viscosity incoming feeds the distribution of the velocity is independent of $\mathrm{Re}_{\mathrm{D}}$ for the considered range of flow rates.

Altering the flow split leads to a significant change in the velocity in the center of the pipe, but the region near the pipe wall is largely unaffected. Also, a large increase in the g-forces experienced by the flow are seen for higher flow splits. However, a higher value of FS also reduces the residence times in the separator due to the higher axial velocities in the center.

In general, changes in boundary conditions in the center, such as changes in flow split or modifications of the geometry of the pick-up tube, have a large influence on the flow field. On the other hand, features in the region closer to the pipe wall, such as the flow straightener, barely have an impact in upstream direction.

The effects of alternative vane designs are approximated by imposing the distribution of the axial and azimuthal velocity on a cross-flow plane just aft of the location of the trailing edge of the vanes. Comparison of the results for the vane-less geometry with the ones for the full geometry shows that this is an accurate approximation. The vane-less geometry, for which it is easier to generate the mesh and is less computationally expensive, can be used in the design stage to determine the required cross-sectional velocity distribution just aft of the vanes and therefore the criteria for vane design. A reduction of the swirl leads to a smaller difference between the maximum and the minimum in the radial distribution of the axial velocity. Reducing the swirl further will eventually lead to a V-shaped distribution of the axial velocity. However, for the present separator the W-shaped distribution of the axial velocity is observed for the range of required azimuthal velocities. The location at which the azimuthal velocity is maximal moves inwards for decreasing swirl. Nevertheless, compared to the axial velocity, the change in azimuthal velocity for different levels of swirl can be more easily anticipated. 
It has been shown that the swirling flow occurring in the present separator design is complex. Changes in flow parameters often lead to substantial changes in the velocity distributions. The dependance of the flow on these parameters is not straightforward. Additional simulations are required to determine this dependency. 
CHAPTER 5

Results for two-phase flow

In this chapter the results of oil-water flow using the two-fluid model are discussed. First, the modeling of the interaction between the two phases is analyzed. Subsequently, the results for two-phase flow are compared to the solution of the single-phase flow for the standard case of a flow rate of $Q=56.5 \mathrm{~m}^{3} / \mathrm{h}$ and a flow split $\mathrm{FS}=0.3$. Finally, the results of the study of the dependency of the flow solution on parameters like the inlet oil volume fraction $\alpha$, the flow split FS and droplet diameter $d$ are discussed and compared to measurements.

\subsection{Introduction}

Much work has been carried out on the numerical simulation of single-phase flow in separators, such as hydrocyclones [28, 49, 55, 78]. However, for multi-phase flow fewer literature sources are available and most are concerned with gas-liquid separation [20,50]. As indicated in chapter 2, two-phase flow is much more complex than single-phase flow. Therefore, it is more difficult to obtain models which describe the behavior of the twophase flow adequately.

For the two-phase flow simulations the same computational mesh is used as for the

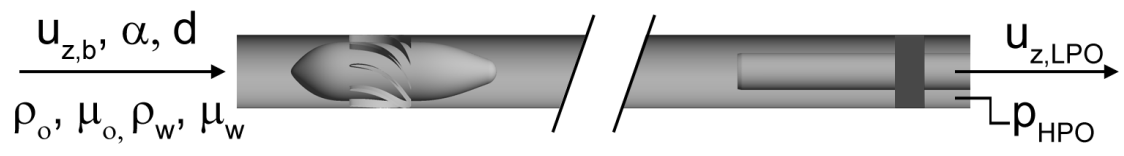

Figure 5.1: Boundary conditions used for simulation of two-phase flow. 
single-phase flow. The boundary conditions for the two-phase flow simulations are shown in figure 5.1. At the inlet the velocity of the oil-water mixture is imposed. The oil and water velocities are identical to $u_{z, b}$. In addition, the oil volume fraction $\alpha_{\text {inlet }}$ is set at the inlet. As before the reference pressure is set to $0 \mathrm{~Pa}$ at the HPO. To control the flow split FS the boundary condition at the LPO is employed. The mass flow at the LPO can no longer be used to determine this condition, because the mass flow depends on the composition of the mixture, which is now an outcome of the simulation. Therefore, a normal velocity $u_{z, L P O}$ is imposed to fix the volume flow rate through the LPO. Only the axial component of the velocity is set, while the radial and azimuthal velocity components are part of the solution. Consequently, the swirling flow is not suppressed by the boundary condition at the LPO.

The input parameters given in table 5.1 are used in the numerical simulations of the flow through the separator discussed in this chapter. Other parameters may vary for different simulations and complete lists of input parameters will be specified in each section. The time step is occasionally reduced slightly when convergence slowed down. The RMS residuals need to converge below $2.5 \times 10^{-6}$. The time-averaging procedure is identical to the one used for single-phase flow. The two-phase flow simulations need to run for a considerably longer simulation time than the single-phase counterparts in order to obtain the oil volume fraction distribution of the operational state.

The number of non-dimensional parameters is higher for two-phase flow due to the increased number of variables. Now seven non-dimensional numbers can be found. The non-dimensional numbers describing the two-phase flow are the length ratio L', the flow split FS, the Reynolds number $\mathrm{Re}_{\mathrm{D}}$, the trailing edge swirl angle $\mathrm{Sw}$, the oil volume fraction $\alpha$, the ratio of the densities $\rho_{o} / \rho_{w}$ and the ratio of the viscosities $\mu_{o} / \mu_{w}$. For two-phase flow $\operatorname{Re}_{\mathrm{D}}$ is based on the mixture viscosity $\mu_{m}$ given by [53]

$$
\mu_{m}=\mu_{w}(1-\alpha)^{-2.5 \frac{\mu_{o}+2 / 5 \mu_{w}}{\mu_{o}+\mu_{w}}}
$$

and the mixture density $\rho_{m}$ is given by

$$
\rho_{m}=\alpha \rho_{o}+(1-\alpha) \rho_{w}
$$

Furthermore, just as L' the ratios of the densities and the viscosities are fixed in the remainder of the present work. In later sections the FS and $\alpha$ will be varied.

\begin{tabular}{|ll||lll|}
\hline$u_{z, b}=2.00 \mathrm{~m} / \mathrm{s}$ & $\rho_{o}$ & $=881 \mathrm{~kg} / \mathrm{m}^{3}$ \\
$\Delta t$ & $=0.5 \mathrm{~ms}$ & $\rho_{w}$ & $=1067.8 \mathrm{~kg} / \mathrm{m}^{3}$ \\
$\mu_{o}$ & $=19.4 \times 10^{-3}$ Pa.s & $\sigma$ & $=0.021 \mathrm{~N} / \mathrm{m}$ \\
$\mu_{w}=1.183 \times 10^{-3}$ Pa.s & $p_{H P O}=0 \mathrm{~N} / \mathrm{m}^{2}$ \\
\hline
\end{tabular}

Table 5.1: Input parameters used for two-phase flow simulations. 


\subsection{Evaluation of the modeling}

Due to the complexity of two-phase flow the modeling will be based on assumption and approximations. It is therefore useful to examine the various models available in Ansys CFX 14.0 in order to obtain an indication of their applicability to the flow in the separator.

\subsubsection{Evaluation of drag law ${ }^{1}$}

Since the influence of the drag law in the multiphase results is of prime importance, it is analyzed more closely. Ishii and Zuber [53] compared the results for two-phase flow attained with their correlation

$$
C_{D}=\frac{24}{R e_{d}}\left(1+0.15 R e_{d}^{0.687}\right)(1-\alpha)^{-2.5 \frac{\mu_{o}+2 / 5 \mu_{w}}{\mu_{o}+\mu_{w}}}
$$

with experimental data and found very favorable agreement. Another evaluation of different drag law correlations has been carried out by Rusche [92] using available experimental data from a wide range of systems and flow conditions. To characterize the experimental data for liquid-liquid flows the Archimedes number Ar is used.

$$
\operatorname{Ar}=\frac{d^{3} \rho_{w}|\Delta \rho| a}{\mu_{w}^{2}}
$$

Here $\Delta \rho=\rho_{w}-\rho_{o}$ and $a$ is the acceleration experienced by the droplet. In the current application $0<\operatorname{Ar}<100$, for which Rusche found that quite accurate predictions were obtained with the Ishii-Zuber correlation. In the experimental data the volume fraction of the dispersed phase ranged from $\alpha=0.2$ to $\alpha=0.6$. However, in the separator even higher volume fractions are expected near the center. Experiments on emulsion compression have been performed at the WUR by Thomas Krebs in order to investigate the accuracy of the Ishii-Zuber drag law at these high $\alpha$ conditions.

\section{Micro-centrifuge setup}

For the micro-centrifuge experiments at the WUR, a custom-made setup was used. Figure $5.2 \mathrm{~A}$ displays a photo of the experimental setup. The setup consists of a disk, which is connected to a DC motor. The range of accessible rotation frequencies $f_{m c}$ is $1.5 \mathrm{~Hz}<$ $f_{m c}<100 \mathrm{~Hz}$. A sample holder can be mounted on the disk. The distance $r_{m c}$ between the center of this sample holder and the motor axis is $0.10 \mathrm{~m}$. The radial acceleration $a_{r}$ is then given by

$$
a_{r}=\left(2 \pi f_{m c}\right)^{2} r_{m c}
$$

The range of the accelerations is thus $8.88 \mathrm{~m} / \mathrm{s}^{2}<a_{r}<39500 \mathrm{~m} / \mathrm{s}^{2}$, or $0.905<a / g<$ 4020 , when expressed in terms of multiples $a / g$ of the normal gravitational acceleration $g=9.81 \mathrm{~m} / \mathrm{s}^{2}$. For the entire range of $a / g$ the induction time of the motor to reach $95 \%$ of the desired acceleration was smaller than $0.5 \mathrm{~s}$.

\footnotetext{
${ }^{1}$ Part of the results presented in this section have been published in revised form in Krebs et al. [61]
} 
The disk and the motor are enclosed in a protective housing. The entire assembly is placed on a custom-made plate, which is mounted on an inverted optical microscope (Axiovert 200, Zeiss). Three set screws allow to align the sample with the objective of the microscope. A high-speed camera (Y4-S2, IDT Inc.) is connected to the microscope. A software trigger has been used (Motion Pro Studio, IDT Inc), which triggered recording when at least one pixel in the image was not black, indicating that the transparent sample chamber is passing by the microscope. A frame rate of $5000 \mathrm{~Hz}$ and an exposure time of 100 ns were used for the experiments. The recorded images were processed with the program ImageJ [37] using custom-written scripts.

A drawing of the sample chamber, which is placed in the sample holder, is displayed in figure 5.2B. The sample chamber consists of two parts, both made from polydimethylsiloxane (PDMS). The bottom part contains a channel of dimensions $5.25 \mathrm{~mm} \times 1 \mathrm{~mm} \times$ $0.2 \mathrm{~mm}$, which is open at one side. The top part of the sample holder contains no specific features. The parts are made by mold casting using the Sylgard Elastomer Kit, which consists of liquid PDMS and a curing agent [86]. The top and bottom part are chemically bonded after exposure to an oxygen plasma. Before each experiment, the sample chamber
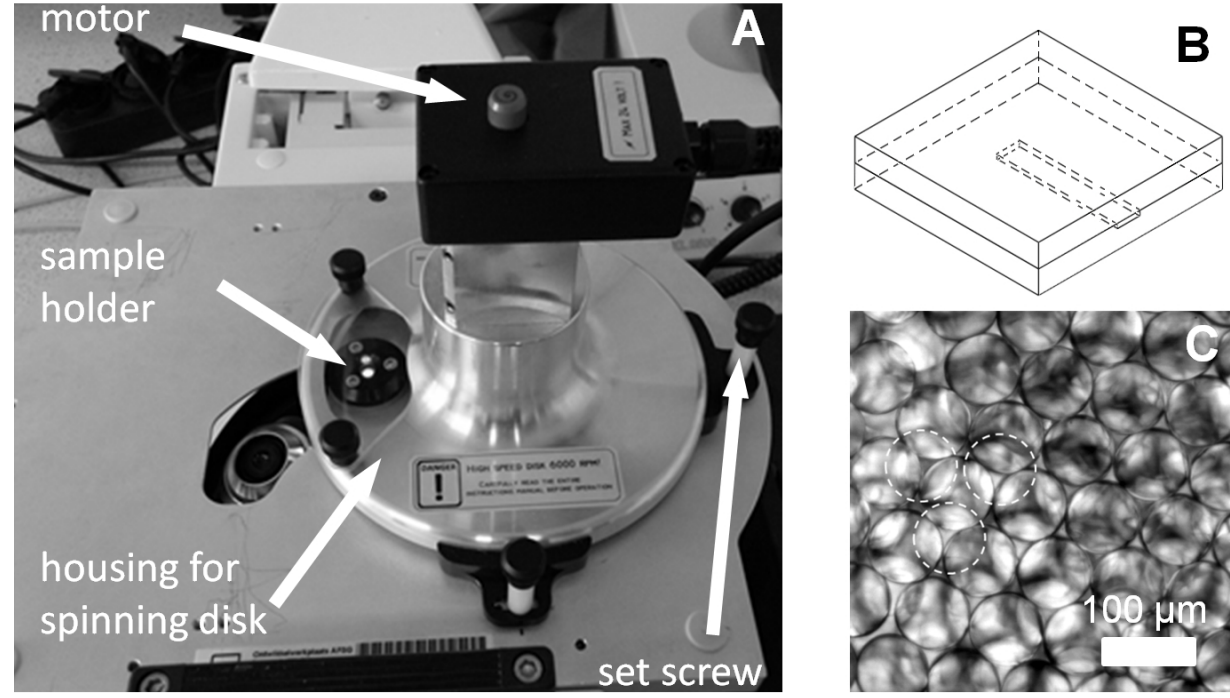

Figure 5.2: Panel A: photograph of the experimental setup at the WUR. Panel B: sketch of the sample chamber used for the centrifuge experiments. The chamber is made from two PDMS disks. The bottom disk contains a channel of dimensions $5.25 \mathrm{~mm} \times 1 \mathrm{~mm} \times 0.2 \mathrm{~mm}$, in which the emulsion is inserted. Panel C: photograph of an oil-in-water emulsion in the sample chamber. The oil phase was Sil180 silicone oil, the aqueous phase a $10 \mathrm{mM}$ SDS solution. The top layer of a bilayer of monodisperse droplets with a mean diameter of $79.3 \mu \mathrm{m}$ is seen. The position of the second droplet layer is indicated by the dotted circles. 
was exposed to an oxygen plasma to render the chamber walls hydrophilic, in order to enable wetting of the channel walls with the continuous phase [86].

\section{Emulsion preparation}

Monodisperse droplets of Sil180 silicone oil in a $10 \mathrm{mM}$ aqueous sodium n-dodecyl sulfate (SDS) solution were produced with a microfluidic T-junction. This experimental setup and its parameters have been described previously by Krebs et al. [59]. At a temperature of $293 \mathrm{~K}$, the density of Sil180 is $931 \mathrm{~kg} / \mathrm{m}^{3}$ and the viscosity equals $10.4 \mathrm{mPas}$. The difference in density between pure water and this oil is $\Delta \rho=69 \mathrm{~kg} / \mathrm{m}^{3}$. The SDS prevented coalescence and thereby stabilized the emulsion for the entire duration of the micro-centrifuge experiments.

An oil-in-water emulsion with a mean droplet diameter $d=79.3 \pm 0.8 \mu \mathrm{m}$ was obtained. The constant diameter of the oil droplets and the fact that the oil phase remains the dispersed phase are in accordance with the assumptions made in the two-fluid model simulations using the Ishii-Zuber model. The emulsion is creamed in a storage flask; droplets are removed from the dense layer using a pipette and are injected into the sample chamber. The mean oil volume fraction $\alpha_{0}$ in the creamed emulsion layer was measured
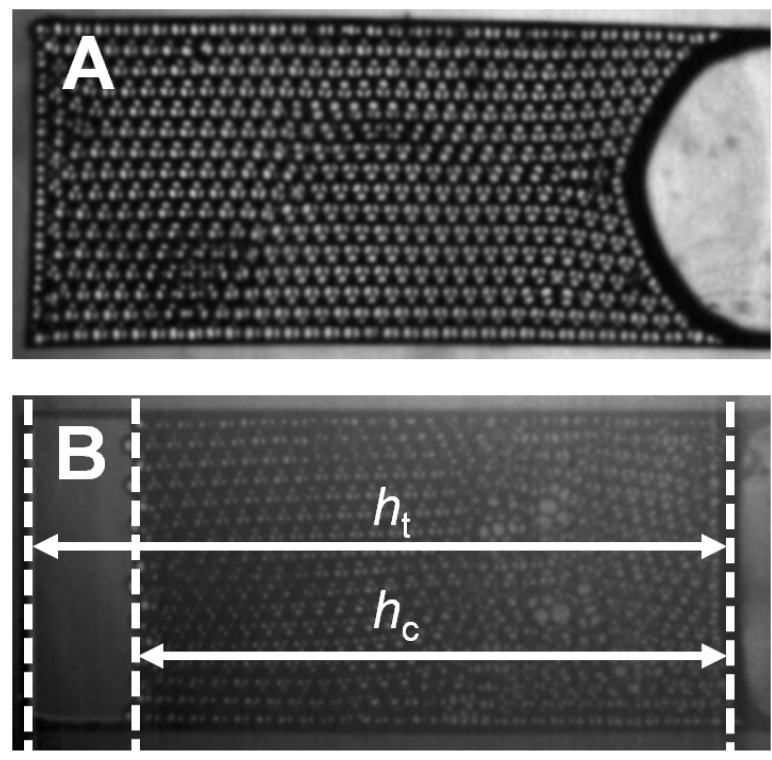

Figure 5.3: Panel A: photograph of the emulsion at rest. The droplet diameter is $d=79.3$ $\mu \mathrm{m}$. An air bubble can be seen on the right. Panel B: Photograph of the same emulsion as in panel A after $5 \mathrm{~s}$ of centrifugation at $231 \mathrm{~g}$. $h_{c}$ and $h_{t}$ indicate the height of the compressed emulsion layer and total height of the liquid column, respectively. Emulsion chamber rotates counterclockwise. 


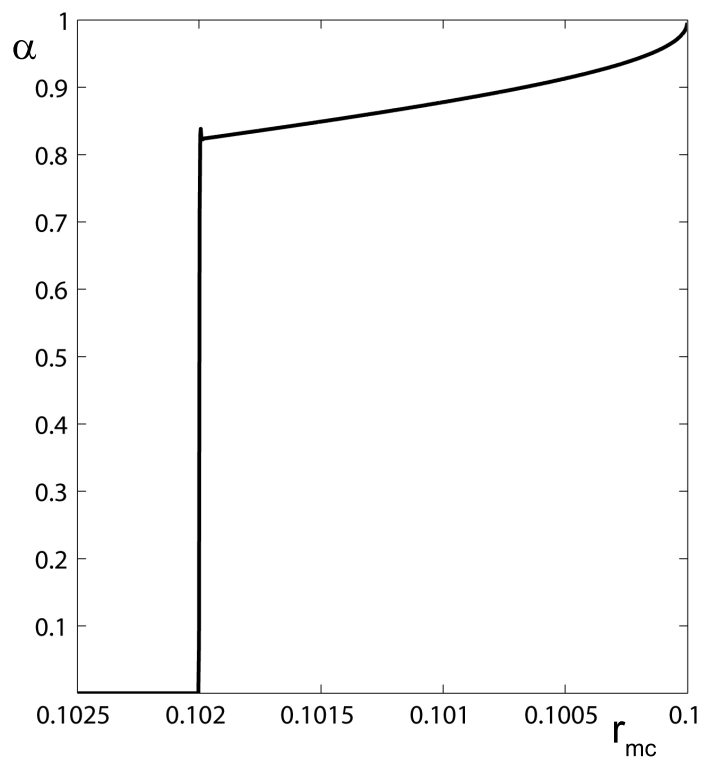

Figure 5.4: Distribution of oil volume fraction $\alpha$ on a line through the center of the sample chamber after $10 \mathrm{~s}$ of centrifugation at $103 \mathrm{~g}$ as obtained from the CFD calculation.

by removing $1 \mu \mathrm{l}$ of sample and counting the number of droplets after spreading of the test volume on a microscope slide. Figure 5.2C displays a photograph of the emulsion in the sample chamber. The droplets have a circular cross section and form a bilayer. The image is focussed on the top layer of droplets. The second layer of droplets is visible, but it appears blurry in figure $5.2 \mathrm{C}$. The position of the droplets in the bottom layer relative to those in the top layer is sketched by the dotted circles.

\section{Numerical simulations}

The governing equations have been solved using the commercial CFD package Ansys CFX. A mesh of 3.3 million hexahedral elements and the use of double precision executables are required to capture the steep gradients in the oil volume fraction and to avoid strong oscillations in the region around that gradient in the numerical solution. A no slip condition is imposed on the walls. The use of a spatially varying centrifugal force leads to numerical oscillations in oil volume fraction. Therefore, a constant body force is used instead. Due the large radius of $\mathcal{O}(100 \mathrm{~mm})$ at which the chamber is placed in the centrifuge, compared to the chamber dimensions of $\mathcal{O}(1 \mathrm{~mm})$, the error made in the centrifugal force is smaller than $1 \%$. All other parameters used in the calculation, such as $d, a$ and $\alpha_{0}$ are matched with the experimental values. As initial condition a homogeneous emulsion with oil volume fraction $\alpha_{0}=0.705$ is used. 


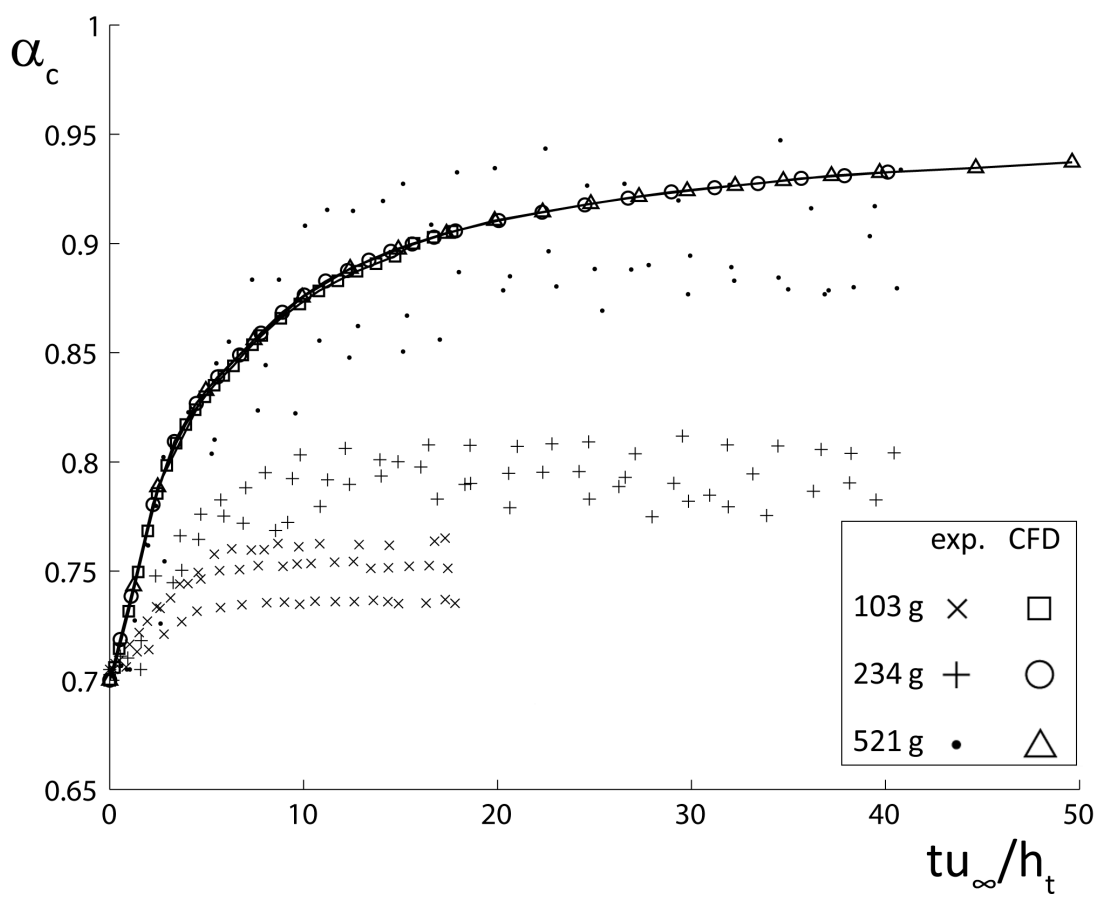

Figure 5.5: Comparison of experimentally and numerically determined averaged oil volume fraction $\alpha_{c}$ in the compressed droplet layer as a function of dimensionless time for $a=103 \mathrm{~g}$ (exp.: $\mathrm{x}$, CFD: $\square), a=234 \mathrm{~g}(\exp .:+$, CFD: $\circ)$ and $a=521 \mathrm{~g}($ exp.: o, CFD: $\triangle$ ) centrifugal acceleration. Three experiments have been carried out for each $a$. Due to the scaling all computed values collapse onto a single curve. $u_{\infty}$ is the terminal velocity of an isolated oil droplet in an infinite domain.

\section{Results and discussion}

Two images from the compression experiments are shown in figure 5.3. Panel A displays the emulsion at rest before centrifugation. Panel B displays a snapshot of the emulsion during centrifugation at $231 \mathrm{~g}, 5 \mathrm{~s}$ after the start of centrifugation. Upon centrifugation, droplets are accelerated towards the axis of rotation, thereby forming a dense layer. From the height of the compressed droplet layer $h_{c}$ and the total height of the liquid column $h_{t}$ the averaged oil volume fraction $\alpha_{c}$ in the compressed layer can be calculated; $\alpha_{c}=$ $\alpha_{0} h_{t} / h_{c}$. For the example shown in figure 5.3B, we obtain $\alpha_{c}=0.83$.

Figure 5.4 shows the distribution of the oil volume fraction $\alpha$ on a line through the center of the sample chamber during centrifugation as obtained from the CFD calculations at $103 \mathrm{~g}$ after $10 \mathrm{~s}$ of centrifugation. Due to the density difference between the two phases, oil is concentrated at the right side of the sample chamber. At the left side of the sample chamber furthest away from the axis of rotation, pure water accumulates. The transition 
between the oil-rich and the oil-free layer is sharp. In the oil-rich layer, $\alpha\left(r_{m c}\right)$ increases with decreasing distance from the rotation axis, and eventually reaches unity. From the profiles of $\alpha\left(r_{m c}\right)$ the average oil volume fraction $\alpha_{c}$ of the oil-rich layer is calculated, which can be used to compare the CFD results with the results of the experiments. For this particular example shown in figure $5.4, \alpha_{c}=0.87$ is obtained.

Figure 5.5 displays $\alpha_{c}$ as a function of time, obtained from the experiments and compares this with the results from the CFD calculations, for 103, 234 and $521 \mathrm{~g}$ centrifugal acceleration. The time is non-dimensionalized by the length scale $h_{t}$ and the terminal velocity $u_{\infty}$ of a single droplet subjected to a constant acceleration in an infinite domain.

$$
u_{\infty}=\frac{\Delta \rho a d^{2}}{18 \mu_{c}}
$$

At the terminal velocity the body forces and the drag force on the droplet are in balance. Note that the terminal velocity depends linearly on the acceleration $a$. The computed values in figure 5.5 collapse onto a single line when the time is non-dimensionalized as $t u_{\infty} / h_{t}$. This clearly does not happen for the experimental data. The agreement between the experimental and numerical curve for $\alpha_{c}(t)$ is not good, nor from a quantitative nor from a qualitative point of view. The calculated values overestimate the experimental values, however, this difference decreases with increasing centrifugal acceleration.

While the curves for $\alpha_{c}(t)$ obtained from CFD continue to increase in time, the measured $\alpha_{c}(t)$ eventually appear to approach asymptotic values. The maximum volume fraction for equal-diameter hard spheres is $\approx 0.74$, which is close to the measured value of the monodisperse dense droplet layer from which the emulsion was sampled. For droplets, higher packing fractions can be achieved, since droplets are deformable. The Bond number Bo is given by

$$
\text { Bo }=\frac{\Delta \rho a d^{2}}{\sigma}
$$

Here $\sigma$ is the interfacial tension. The Bond number Bo compares the magnitude of the body force and that of the interfacial tension force. For the present system, at the smallest acceleration of $103 \mathrm{~g}$, we obtain Bo $\approx 0.11$, which indicates that droplet deformation will take place [44], and that the measured values of $\alpha_{c}$ are physically reasonable. For $a=$ 103,234 and $521 \mathrm{~g}$, Bo $=0.11,0.25$ and 0.55 , respectively, and the measured maximum oil volume fractions $\alpha_{c, \max }=0.75,0.79$ and 0.90 , respectively.

The maximum oil volume fraction $\alpha_{c, \max }$ appears to increase linearly as function of the Bond number Bo as is shown in figure 5.6. The linear fit shown in the figure is given by

$$
\alpha_{c, \max }=0.355 \mathrm{Bo}+0.71
$$

Error bars give standard deviation in experimental results.

Due to the volume averaging procedure carried out in the derivation of the two-fluid model, the oil phase no longer has the characteristics of discrete droplets, but those of a continuous medium. Therefore, the behavior of the emulsion related to the droplet deformation cannot be captured and the calculated oil volume fraction $\alpha$ will eventually approach unity for any non-zero radial acceleration. In addition, as pointed out in chapter 


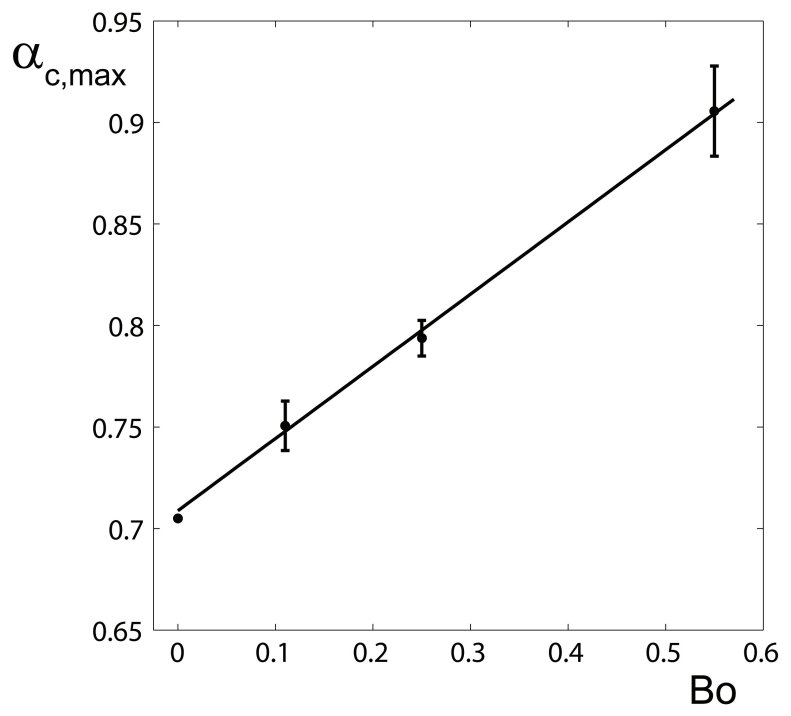

Figure 5.6: Maximum oil volume fraction $\alpha_{c, \max }$ as function of Bond number Bo found in experiment on emulsion compression with linear curve fit: $\alpha_{c, \max }=0.355 \mathrm{Bo}+0.71$. Error bars give standard deviation in experimental results.

2, Taylor [109] assumed spherical droplets in his derivation of the mixture viscosity, while in the present experiments considerable deformation of the droplets are seen. Furthermore, while some deviations from the spherical shape are permitted according to Roscoe [88], his derivation of the mixture viscosity assumes a very wide particle size distribution. In the present numerical simulations, a monodisperse emulsion is used. These differences between the experiments and the assumptions in the derivation of the model also contribute to the observed discrepancies in the results.

In principle the maximum oil volume fraction in the numerical simulations can be limited based on experimental correlation such as given in equation (5.8). However, in that case the numerical method will be tailored to these experiments with emulsions in which coalescence is prevented. In the experimental setup at the TUD coalescence is expected, leading to a different behavior of the emulsion. Therefore imposing a limit on the oil volume fraction may not lead to more accurate results.

The results demonstrate that the Ishii-Zuber drag law should be applied with caution in CFD calculations simulating multiphase flows with high volume fractions of the dispersed phase since large deviations from experiments are seen even for relatively simple flows. 


\subsubsection{Turbulent dispersion ${ }^{2}$}

Preliminary studies with Euler-Lagrangian droplet tracking indicated the importance of modeling the turbulent dispersion for the separation of liquid-liquid mixtures in dilute multi-phase systems. Therefore, these results are discussed here as they imply that turbulent dispersion will also have an effect in the case of denser systems. These computationally inexpensive two-phase flow simulations were carried out in the design phase of the ISE in order to obtain a first estimate on the behavior of the dispersed phase. In these early computations in the design phase a separate oil outlet (LPO) was not present.

\section{Lagrangian droplet tracking method}

A small number of oil droplets was simultaneously released at the inlet and followed through the separator. One-way coupling was used, so there is no influence of the dispersed droplets on the continuous flow field. The one-way coupling is justified as indicated by Crowe [24], since the droplets are small $(d \ll 1 \mathrm{~mm})$, the number of droplets is low $\left(n_{d} \sim\right.$ $\left.10^{3}\right)$, the density difference between the phases is low $\left(\Delta \rho \approx 200 \mathrm{~kg} / \mathrm{m}^{3}\right)$ and viscosity of water is relatively high.

The equation of motion of a droplet is given by [3]:

$$
\begin{aligned}
m_{d} \frac{d\left\langle\mathbf{u}_{\mathbf{d}}\right\rangle}{d t}= & \frac{1}{2} \rho_{c} C_{D} A_{d}\left|\left\langle\mathbf{u}_{c}\right\rangle-\left\langle\mathbf{u}_{d}\right\rangle\right|\left(\left\langle\mathbf{u}_{c}\right\rangle-\left\langle\mathbf{u}_{d}\right\rangle\right) \\
& +\left(m_{d}-m_{c}\right) \mathbf{g} \\
& +\frac{1}{2} m_{c}\left(\frac{d\left\langle\mathbf{u}_{c}\right\rangle}{d t}-\frac{d\left\langle\mathbf{u}_{d}\right\rangle}{d t}\right) \\
& +\frac{m_{c}}{\rho_{c}} \nabla\langle p\rangle
\end{aligned}
$$

Here $m_{d}$ is the mass of the oil droplet, $m_{c}$ is the mass of the displaced water and $A_{d}$ is the effective droplet cross-sectional area. The correlation of Schiller and Naumann [97], equation (2.92), is used for the drag coefficient $C_{D}$. The subsequent terms on the righthand side of equation (5.9) represent: the drag force; the buoyancy force due to gravity; virtual mass force; and the pressure gradient force, respectively. Note that in the virtual mass force the time derivative of the velocity of the water phase is used instead of the material derivative. This corresponds to the implementation of the virtual mass force in CFX 14.0. In addition, the Basset history force is not included. For the present case the history force is assumed to be small relative to the drag force [32].

The motion of small droplets is affected by the turbulent fluctuations of the continuous flow. Turbulent eddies can displace a droplet and therefore frustrate the segregation process. This turbulent dispersion is accounted for by using the model of Gosman and loannides [41]. This model assumes that the droplet is present in and interacts with a single turbulent eddy. The interaction with the eddy is modeled by adding velocity increments $\tilde{\mathbf{u}}_{c}^{\prime}$ to the mean velocity $\left\langle\mathbf{u}_{c}\right\rangle$ of the fluid surrounding the droplet. Velocity increment $\tilde{\mathbf{u}}_{c}^{\prime}$ is an approximation of the turbulent velocity fluctuation $\mathbf{u}_{c}^{\prime}$. The new estimate of the

\footnotetext{
${ }^{2}$ Part of the results presented in this section have been published in revised form in Slot et al. [104]
} 
instantaneous fluid velocity $\left\langle\tilde{\mathbf{u}}_{c}\right\rangle=\left\langle\mathbf{u}_{c}\right\rangle+\tilde{\mathbf{u}}_{c}^{\prime}$ replaces $\left\langle\mathbf{u}_{c}\right\rangle$ and is used in the calculation of the drag force and the relative Reynolds number. The velocity increment is defined as:

$$
\tilde{\mathbf{u}}_{c}^{\prime}=\boldsymbol{\Gamma} \sqrt{2 k / 3}
$$

Here $\boldsymbol{\Gamma}$ is a normal-distributed random vector, which ensures that the velocity increments are randomly distributed over the different directions. In order to conserve kinetic energy $\boldsymbol{\Gamma}$ is a unit vector, that is $\|\boldsymbol{\Gamma}\|=1$. Furthermore $k$ is the local turbulent kinetic energy. Note that $\sqrt{2 k / 3}$ is the magnitude of the velocity fluctuations in isotropic turbulent flow. The velocity increment $\tilde{\mathbf{u}}_{c}^{\prime}$ is constant during the droplet-eddy interaction time $\tau_{\text {int }}$. This is the minimum of two timescales: the eddy lifetime $\tau_{e}$ and transit time $\tau_{t r}$. The characteristic length scale of the eddy is

$$
l_{e}=\frac{C_{\mu}^{1 / 2} k^{3 / 2}}{\epsilon}
$$

Here $C_{\mu}$ is a turbulence constant equal to 0.09 and $\epsilon$ is the turbulent dissipation rate. The eddy lifetime is then given by

$$
\tau_{e}=\frac{l_{e}}{\sqrt{2 k / 3}}
$$

The transit time is defined as the time required for the droplet to transverse the eddy. This time scale is estimated from the solution of the simplified equation of motion of the

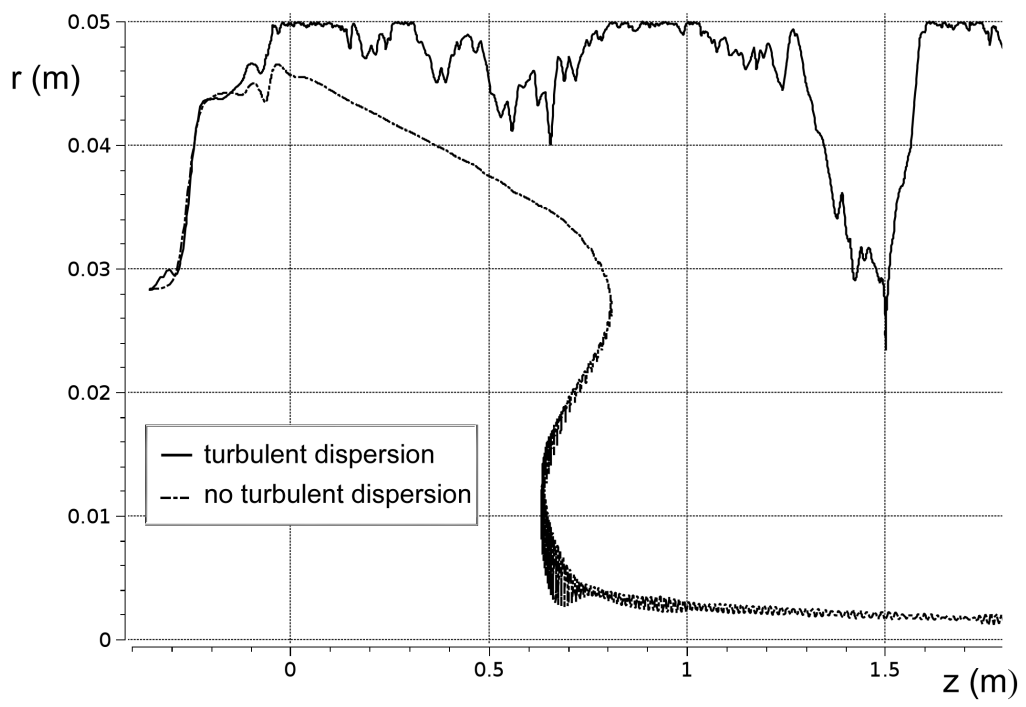

Figure 5.7: Radial distance from pipe axis of a $50 \mu \mathrm{m}$ diameter droplet as function of axial distance $z$, calculated with (solid) and without (dash-dotted) turbulent dispersion. $\operatorname{Re}_{\mathrm{D}}=$ $1.81 \times 10^{5}$ and $\mathrm{Sw}=6.72$. 
droplet

$$
\tau_{t r}=-\tau_{d} \ln \left(1-\frac{l_{e}}{\tau_{d}\left|\left\langle\tilde{\mathbf{u}}_{c}\right\rangle-\left\langle\mathbf{u}_{d}\right\rangle\right|}\right)
$$

Here $\left\langle\mathbf{u}_{d}\right\rangle$ is the mean velocity of the droplet and $\tau_{d}$ is the droplet response time, given by:

$$
\tau_{d}=\frac{\rho_{d} d^{2}}{18 \mu_{c}}
$$

The product $\tau_{d}\left|\left\langle\tilde{\mathbf{u}}_{c}\right\rangle-\left\langle\mathbf{u}_{d}\right\rangle\right|$ is an estimate of the distance the droplet travels before it adjusts to the characteristic velocity of the eddy. This needs to be larger than $l_{e}$, if the droplet is to cross the eddy at all. When the droplet-eddy interaction time is reached, new values of $\tilde{\mathbf{u}}_{c}^{\prime}, l_{e}$ and $\tau_{\text {int }}$ are calculated based on local values of the mean velocity $\left\langle\mathbf{u}_{c}\right\rangle$, the turbulent kinetic energy $k$ and turbulent dissipation rate $\epsilon$ at the position of the droplet.

Equation (5.9) is integrated in time using the velocity field that is calculated for the present time step. Equation (5.9) is integrated using 250 integration steps per cell of the mesh. This is higher than the default setting but is required to yield results which are independent of the number of integration steps. At every droplet position the forces are determined by interpolating linearly between values at the the surrounding vertices. Integration is continued until one time step is completed. At a given time 200 droplets are simultaneously released at the inlet. The droplets are randomly distributed over the crosssectional area of the inlet. The droplets have a density $\rho_{d}$ of $800 \mathrm{~kg} / \mathrm{m}^{3}$, the water has a density of $997 \mathrm{~kg} / \mathrm{m}^{3}$. Three calculations have been carried out for a mono-dispersed droplet size of 50,75 and $100 \mu \mathrm{m}$. Due to computational constraints a somewhat coarser mesh of $1.25 \times 10^{6}$ hexahedral elements was used for the droplet trajectory calculations.

\section{Droplet trajectories}

The effect of the turbulent dispersion is illustrated in figure 5.7. This figure shows the radial distance from the pipe axis of a $50 \mu \mathrm{m}$ diameter droplet as function of axial distance $z$, which is calculated with and without the turbulent dispersion model. The result of the simulation with turbulent dispersion taken into account shows an erratic trajectory of the oil droplets through the separator. As the trajectory indicates, droplets can also be transported away from the center after being segregated. In contrast, the simulation

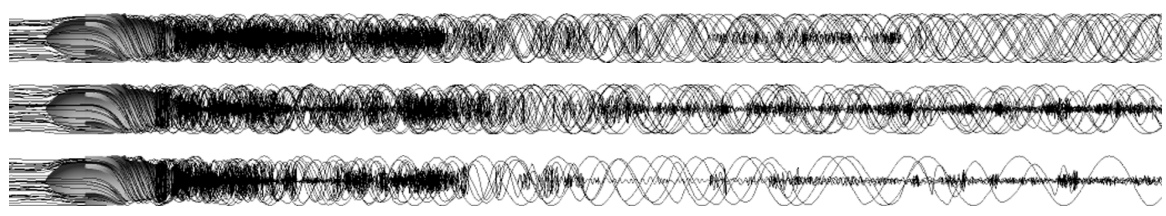

Figure 5.8: Trajectories of droplets of 50 (top), 75 (middle) and $100 \mu \mathrm{m}$ (bottom) diameter at $t=2.8 \mathrm{~s}$ after release at inlet. For each droplet size 40 trajectories are shown. $\operatorname{Re}_{\mathrm{D}}=1.81 \times 10^{5}$ and $\mathrm{Sw}=6.72$. 
without the turbulent dispersion modeling shows a relatively smooth motion toward the center of the pipe with only small-amplitude oscillations near the center.

Figure 5.8 shows trajectories of 50,75 and $100 \mu \mathrm{m}$ diameter droplets at $t=2.8 \mathrm{~s}$ after release of the droplets at the inlet. For each diameter size 40 randomly selected trajectories of the 200 computed trajectories are shown. After $t=2.8 \mathrm{~s}$ not all droplets have reached the outlet. Some droplets have segregated towards the center of the pipe and are slowly transported downstream. Away from the center, the axial velocity is much higher and there the droplets move quickly to the outlet. The figure clearly demonstrates the influence of the droplet size on the separation. A large number of trajectories of the smaller droplets remain near the wall and no apparent inward motion is observed. A detailed view on two droplet trajectories is shown in figure 5.9. The figure shows the radial distance from the pipe axis of a 50 and a $100 \mu \mathrm{m}$ diameter droplet as function of the axial distance $z$. The effect of the turbulent dispersion decreases with increasing droplet diameter due to the higher droplet response times of the larger droplets. However, the $100 \mu \mathrm{m}$ diameter droplets can still be considered small since both 50 and $100 \mu \mathrm{m}$ diameter droplets experience radial in- and outward displacement of roughly the same order of magnitude and frequency. The larger centrifugal force on the $100 \mu \mathrm{m}$ diameter droplets is responsible for the better segregation of these droplets. Close inspection of the $100 \mu \mathrm{m}$ droplet trajectory reveals the presence of the annular reversed flow region, since around $z=1.1 \mathrm{~m}$ the droplet briefly moves upstream.

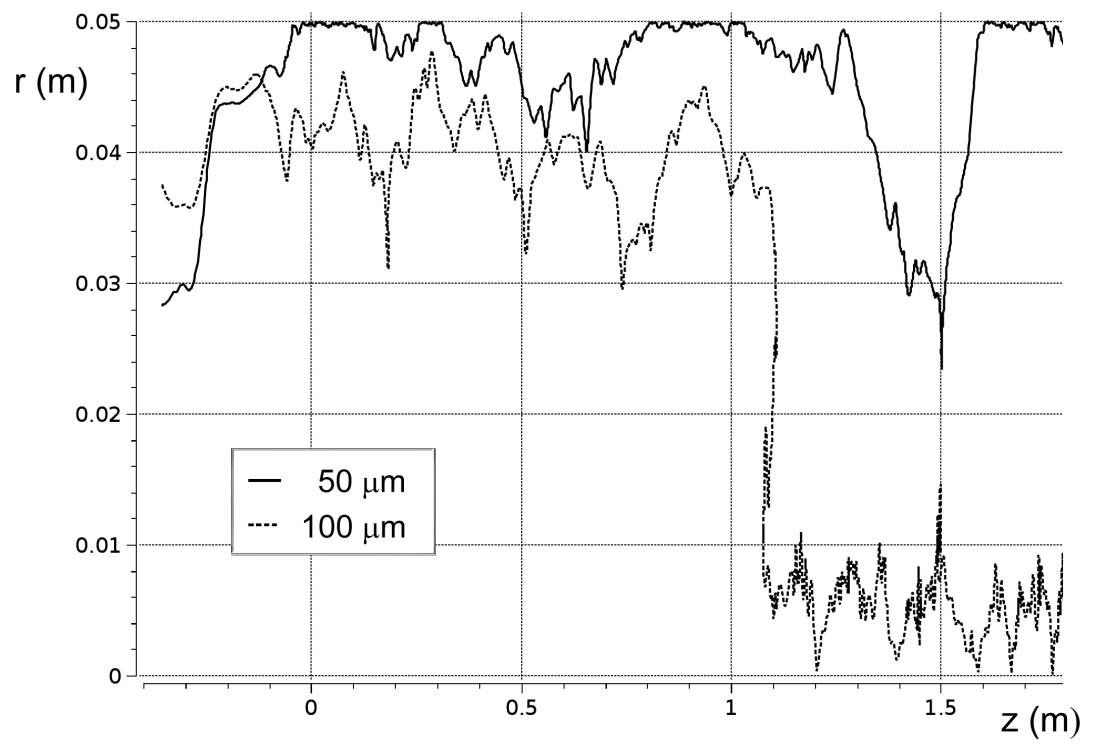

Figure 5.9: Radial distance from pipe axis of a $50 \mu \mathrm{m}$ diameter droplets (solid) and a $100 \mu \mathrm{m}$ diameter droplet (dotted) as function of axial distance $z$. $\operatorname{Re}_{\mathrm{D}}=1.81 \times 10^{5}$ and $\mathrm{Sw}=6.72$. 


\section{Two-fluid turbulent dispersion model}

The droplet tracking results indicate that turbulent dispersion is an important effect and it should be incorporated in the two-fluid modeling. This turbulent dispersion model has been discussed in section 2.4.2. Unfortunately, the turbulent dispersion model of Burns et al. [14] assumes isotropic turbulence, while the turbulence inside the separator is clearly anisotropic.

The turbulent dispersion model by Burns et al. [14] contains an adjustment parameter $C_{1}$, which can be altered to tune the model. The default value of $C_{1}$ is 1.0 . To assess the sensitivity of the result to the adjustment parameter in the turbulent dispersion model of Burn et al. [14], simulations were run for three values of $C_{1}: 0,0.5$ and 1 . The magnitude of the turbulent dispersion depends linearly on the drag coefficient $C_{D}$. For these simulation the Schiller-Naumann drag law, given in equation (2.92), is used to define $C_{D}$. In other two-phase flow simulations the Ishii-Zuber drag law is applied because of the high volume fraction of the dispersed phase. However, the observed trends with regards to $C_{1}$ in the simulations with the Schiller-Naumann drag relation also hold for different drag laws. Figure 5.10 shows the predicted time-averaged oil volume fraction $\alpha$ on the cross-sectional plane through the axis of the separator. In the results presented in figure 5.10 the droplet size $d=100 \mu \mathrm{m}$ and $\operatorname{Re}_{\mathrm{D}}=0.86 \times 10^{5}$. Moreover, figure 5.11 shows the radial distribution of the oil volume fraction for the three cases with varying $C_{1}$ on a line through the center of the pipe in the plane $z=1.5 \mathrm{~m}$.

Clearly, the impact of using the model of turbulent dispersion is large. The case without turbulent dispersion shows pure oil in the center, while the other cases show a decrease in oil volume fraction along the centerline in downstream direction. The diffusion of the oil volume fraction is very likely to be too strong, and therefore the oil volume fraction is underestimated. The reason for this strong dispersion is the underlying assumption that the turbulent transport is described by the eddy diffusivity hypothesis and the increased radial diffusion of the oil volume fraction is similar to the increased radial diffusion of momentum observed when using two-equation turbulence models for this type of swirling

$\begin{array}{llllll} & & & & \text { Oil volume fraction (-) } \\ 0.00 & 0.25 & 0.50 & 0.75 & 1.00 & \end{array}$

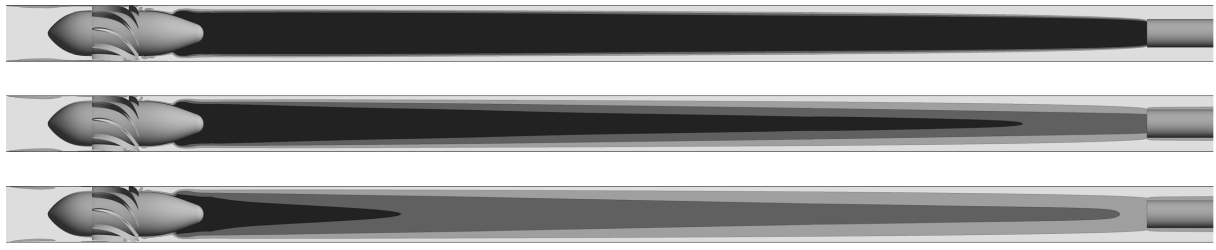

Figure 5.10: Time-averaged oil volume fraction $\alpha$ on a plane through the axis of separator for different constants $C_{1}$ in equation (2.99): $C_{1}=0$ (top), $C_{1}=0.5$ (middle) and $C_{1}=1$ (bottom). In axial direction the oil volume fraction can clearly be seen to decrease when turbulent dispersion is enabled. $\mathrm{FS}=0.30, \operatorname{Re}_{\mathrm{D}}=0.86 \times 10^{5}, \mathrm{Sw}=6.61, d / D=1.00 \times 10^{-3}, \alpha_{\text {inlet }}=0.25$. 


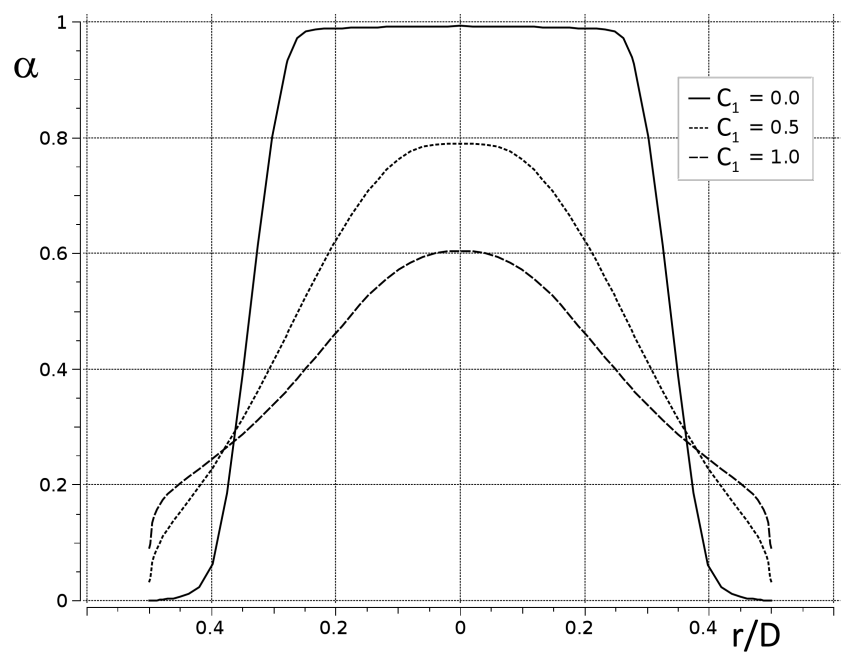

Figure 5.11: Radial distribution of time-averaged oil volume fraction $\alpha$ for $C_{1}=0$ (solid), $C_{1}$ $=0.5$ (dotted) and $C_{1}=1$ (dashed) on a line through the center of the pipe in the plane $\mathrm{z}=$ $1.5 \mathrm{~m}$. Dispersion of oil phase can clearly be seen when turbulent dispersion is enabled. $\mathrm{FS}=$ $0.30, \operatorname{Re}_{\mathrm{D}}=0.86 \times 10^{5}, \mathrm{Sw}=6.61, d / D=1.00 \times 10^{-3}, \alpha_{\text {inlet }}=0.25$.

flow [78]. The current model seems inadequate for swirling flow.

In light of the results for the dilute two-phase flow, turbulent dispersion may be an significant feature of the flow and it needs to be modeled accurately. However, the higher oil volume fractions may dampen the turbulence and the possible formation of an oil continuous layer will lead to a different effect of the turbulence on the oil phase compared to the case of dilute two-phase flow. Further research, both experimental and modeling, is required to shed light on the significance of turbulent dispersion for dense systems and the appropriate modeling of turbulent dispersion that can be used for numerical simulations. Currently, a model for anisotropic turbulent dispersion model is not available within CFX 14.0. The present turbulent dispersion model is thought to over-predict the diffusion of oil and accurate tuning of $C_{1}$ is not known. Therefore, the turbulent dispersion model is not used in subsequent simulations of two-phase flow, as its use presumably results in inaccurate flow solutions.

\subsubsection{Virtual mass force}

A numerical simulation has been carried out in which the virtual mass force is enabled in order to investigate its influence on the flow field. This influence was expected to be small, however, the virtual mass force is expected to slow down the radially inward motion of the oil and will therefore negatively affect the separation.

Unfortunately, the use of the virtual mass force as implemented in CFX 14.0 leads to problems. In figure 5.12 the distribution of the oil volume fraction on the wall just aft 
of the ISE is plotted. The vanes can be seen on the left in the figure. Upstream of the vanes the oil volume fraction at the wall is increasing to a value just above $\alpha=0.25$. The elongated streaks of oil-rich fluid on the pipe wall are oriented almost perpendicular to the direction of the swirling flow and the streaks are advected downstream. The azimuthal velocity is non-zero at the location of these streaks and it is unclear what is holding the oil at the wall. In addition, large amplitude wiggles in velocity and large slip velocities between the phases are seen near the wall. Based on equation (2.101) of the virtual mass force this behavior is unexpected and thought to be a numerical artefact. Enabling double precision solver executables or time step reductions did not alleviate the problem. Also difficulties in the convergence of the residuals were encountered. The virtual mass force therefore is considered not to be suited for the present application and it is not used in further numerical simulations.

\subsubsection{Lift force}

Two simulations have been carried out to evaluate the effect of enabling the Saffman-Mei lift force $[93,94,77]$. One with the lift force enabled and the other without the lift force, in all other respects the input and computational grid for the two simulations are identical. The results show that the lift force is of minor influence on the flow solution. Although the vorticity is high, the velocity difference in most of the separator is quite small. The low slip velocity is due to the high drag force experienced at high volume fraction of the dispersed phase. This decreases the lift force, which is given in equations (2.102) to (2.107).

The axial velocity is only significantly affected in the center of the pipe. The numerical simulation with the lift force showed a lower velocity in the center. This difference in velocity increases in downstream direction. At $z=1.50 \mathrm{~m}$, the maximum difference is about $5 \mathrm{~cm} / \mathrm{s}$, as can be seen in figure 5.13 . The azimuthal velocity component is virtually identical with or without the lift force. The largest differences are of the order of $1 \%$ of the magnitude of the maximum azimuthal velocity. Minor differences in the distribution of the oil volume fraction are seen, resulting in insignificant changes in the separation

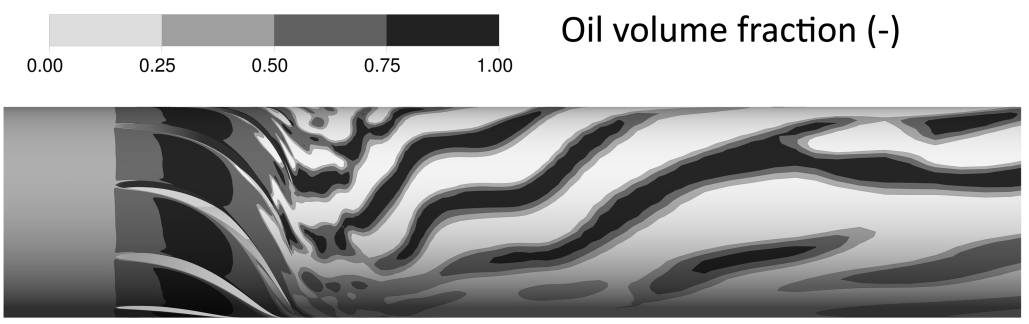

Figure 5.12: Distribution of oil volume fraction $\alpha$ on pipe wall just downstream of ISE. Oil streaks appear at the wall when the virtual mass force is taken into account. Location of the vanes can be seen on the left. $\mathrm{FS}=0.30, \mathrm{Re}_{\mathrm{D}}=0.86 \times 10^{5}, \mathrm{Sw}=6.61, d / D=1.00 \times 10^{-3}$, $\alpha_{\text {inlet }}=0.25$. 


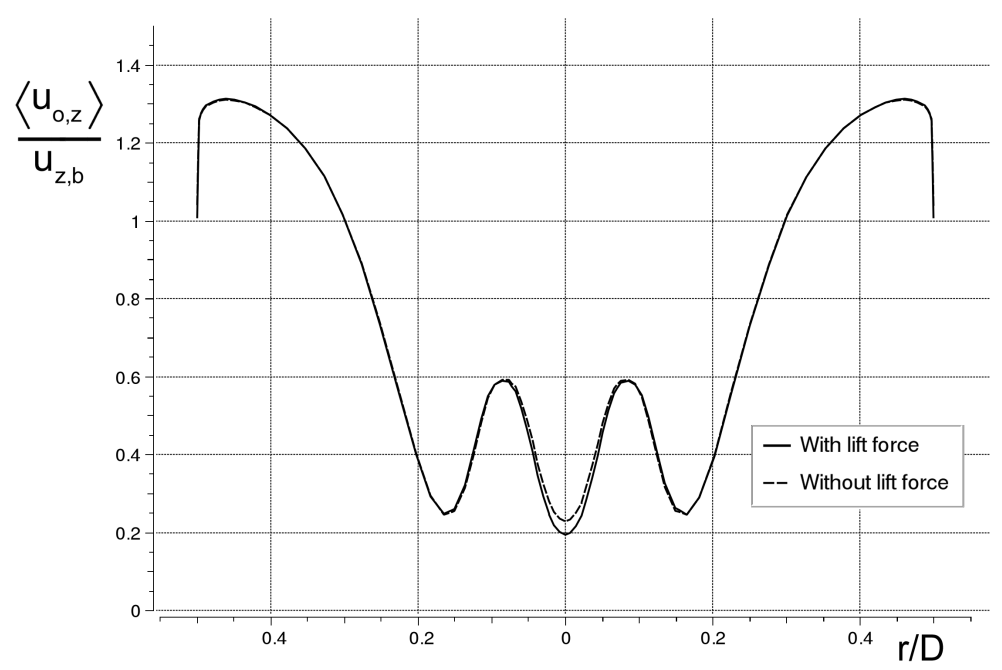

Figure 5.13: Comparison of the radial distribution of the time-averaged axial component of the oil velocity on a line through the center of the pipe in the plane $z=1.50 \mathrm{~m}$ for cases with (solid) and without (dashed) lift force enabled. $\mathrm{FS}=0.30, \mathrm{Re}_{\mathrm{D}}=0.86 \times 10^{5}, \mathrm{Sw}=6.61, d / D$ $=1.00 \times 10^{-3}, \alpha_{\text {inlet }}=0.25$.

performance.

Since the lift force is not important, it could be neglected in the numerical simulations. However, the model for the lift force is readily available within Ansys CFX and including it will incorporate more physics into the model. Therefore, the lift force will be used in subsequent numerical simulations.

\subsubsection{Conclusions}

The results of the numerical flow simulations obtained with the Ishii-Zuber drag law show large differences compared to experimental results, even for a relatively simple flow without turbulence and without phase inversion. Thus, the numerical solutions obtained with the Ishii-Zuber drag law should be critically reviewed and the end-user should be aware of the deviations from reality due to incomplete modeling. However, the Ishii-Zuber relation for the drag law is the best of the currently available models and is therefore employed in the present project.

Turbulent dispersion of the oil droplets is expected to be an important aspect of the flow. In the present project this flow feature cannot be incorporated accurately due to inadequacy of the model for turbulent dispersion in a swirling flow. Further research effort is necessary to develop and to implement appropriate modeling for turbulent dispersion in dense swirling two-phase flows.

In addition, it was shown that the use of the virtual mass force leads to unphysical oil distributions at the pipe wall. Therefore, the use of the virtual mass force is not 


\begin{tabular}{|c|c|c|c|c|c|}
\hline$d$ & $=$ & $100 \mu \mathrm{m}$ & $\alpha_{\text {inlet }}$ & $=$ & 0.25 \\
\hline FS & $=$ & 0.30 & $\mu_{o}$ & $=$ & $19.4 \times 10^{-3}$ Pa.s \\
\hline $\operatorname{Re}_{\mathrm{D}}$ & $=$ & $0.86 \times 10^{5}$ & $\mu_{w}$ & $=$ & $1.183 \times 10^{-3}$ Pa.s \\
\hline Sw & $=$ & 6.61 & $\rho_{o}$ & $=$ & $881 \mathrm{~kg} / \mathrm{m}^{3}$ \\
\hline$u_{z, b}$ & $=$ & $2.00 \mathrm{~m} / \mathrm{s}$ & $\rho_{w}$ & $=$ & $1067.8 \mathrm{~kg} / \mathrm{m}^{3}$ \\
\hline
\end{tabular}

Table 5.2: Input parameters used for two-phase flow simulations discussed in section 5.3.

recommended. Fortunately, the effect of the virtual mass force is presumed to be small due the relatively small density differences between the water phase and the oil phase. Since the density difference is small the lift force is thought to be of minor importance as well. However, the lift force is incorporated in the flow simulations since the model of the lift force is readily available.

Furthermore, from experiments it is known that for a certain range of volume fractions of the dispersed phase and droplet size the viscosity of the mixture can be much higher than the viscosity of the individual components [5]. Such a flow feature may be incorporated into a two-phase mixture model. In the mixture model the two-phase mixture is described by a single velocity field and the evolution of the oil volume fraction needs to be modeled, for example by a transport equation [71]. However, within in the framework of the twofluid model this is not possible. The mixture viscosity can indeed be incorporated into the drag law, but in this way it only affects the slip velocity. The velocity distribution of the two phases is coupled by the generalized interfacial drag term $M_{k, i}$, but the computation of these velocity distributions uses the individual viscosities $\mu_{o}$ and $\mu_{w}$. Flow features such as a peak in viscosity depending on the flow condition cannot be represented by the two-fluid model.

\subsection{Results for two-phase flow}

The numerical simulation of the two-phase flow for nominal conditions will now be discussed. The input parameters for the two-phase flow simulation are given in table 5.2. Note that here the oil has different material properties than the oil used in chapter 4. The reason is that in the present chapter the conditions for the numerical simulations

\begin{tabular}{lllllll}
\hline & & & & & & Oil volume fraction
\end{tabular}

Figure 5.14: Distribution of time-averaged oil volume fraction $\alpha$ on plane through the axis of the separator. Large hold-up of oil is seen and maximum $\alpha$ is just above 0.80 . $F S=0.30, \operatorname{Re}_{\mathrm{D}}$ $=0.86 \times 10^{5}, \mathrm{Sw}=6.61, d / D=1.00 \times 10^{-3}, \alpha_{\text {inlet }}=0.25$. 
are matched with the ones of the experiments at the TUD. The velocity field reaches the operational state in about 2 to 3 simulation seconds. However, the evolution of the oil volume fraction typically needs an additional 1 to 2 seconds of simulation time. In addition, two sets of governing equations for the phases need to be solved now. This leads to substantial computational times for two-phase flow simulations. Therefore, the total number of numerical simulations of two-phase flow discussed in this chapter is limited.

\subsubsection{Separation performance}

Generally the slip velocity between the water and the oil phase is quite small. Only in the outer region, close to the pipe wall, slip velocities between the two phases of 0.10 to $0.15 \mathrm{~m} / \mathrm{s}$ are seen. This is mostly due to the radial component of the slip velocity, which drives the separation. The axial and azimuthal components of the slip velocity of roughly $0.05 \mathrm{~m} / \mathrm{s}$, are about $1 \%$ of the magnitude of the respective velocity components of the oil and water phase. For $r<35 \mathrm{~mm}$, the slip velocity is smaller than $5 \times 10^{-3} \mathrm{~m} / \mathrm{s}$.

The distribution of the oil volume fraction $\alpha$ is of prime importance for the separation performance of the separator. The distribution of the oil volume fraction can be seen in figure 5.14. Oil quickly moves away from the outer region of the pipe towards the center, where a large region of oil-rich fluid is seen. The hold-up of oil is defined as the volume-averaged oil volume fraction $\alpha$ in the total volume of the separator. In the present

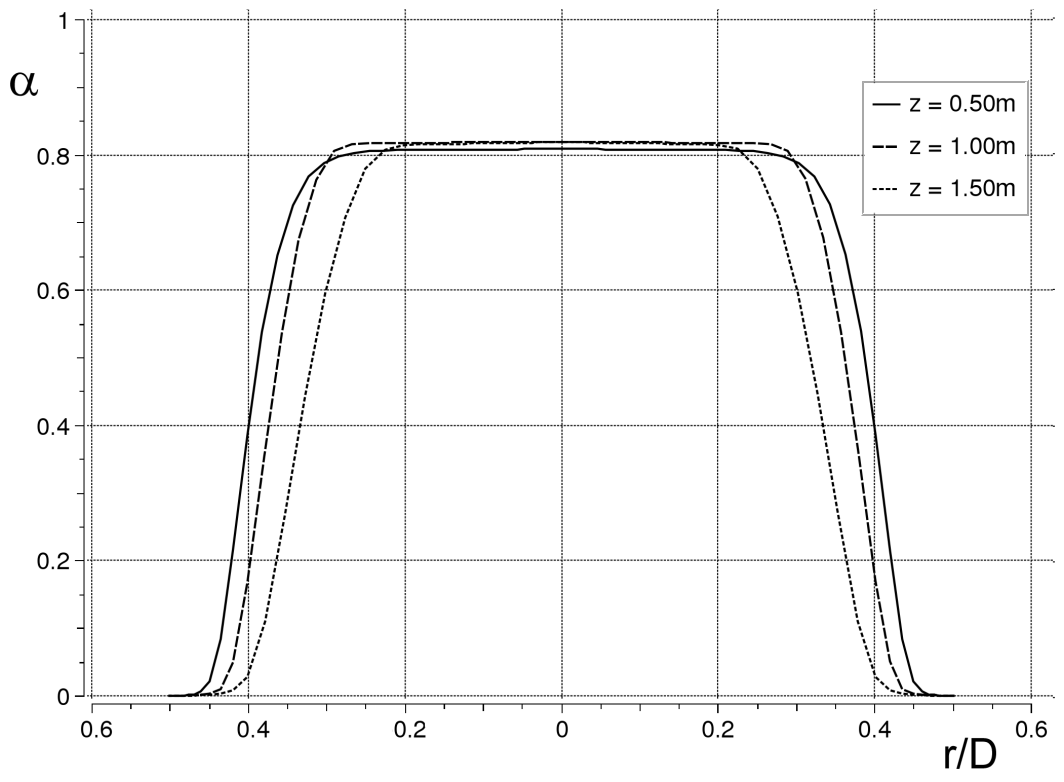

Figure 5.15: Radial distribution of the time-averaged oil volume fraction $\alpha$ in number of crossflow planes. In downstream direction the oil-rich core becomes more narrow and the maximum $\alpha$ increases to a value just above $0.80 . \mathrm{FS}=0.30, \mathrm{Re}_{\mathrm{D}}=0.86 \times 10^{5}, \mathrm{Sw}=6.61, d / D=1.00 \times 10^{-3}$, $\alpha_{\text {inlet }}=0.25$. 


\begin{tabular}{|c|c|c|c|c|c|c|c|}
\hline & $\alpha_{H P O}$ & $\alpha_{L P O}$ & hold up & $\eta_{1}$ & $\frac{\Delta Q_{o}}{Q_{o, \text { inlet }}}$ & $\eta_{2}$ & $\frac{1}{1-F S} \frac{\Delta Q_{o}}{Q_{o, \text { inlet }}}$ \\
& $(\%)$ & $(\%)$ & $(\%)$ & $(\%)$ & $(\%)$ & $(\%)$ & $(\%)$ \\
\hline \hline CFD & 4.9 & 72 & 42 & 86 & 0.56 & 80 & 0.80 \\
Exp. & 16 & 46 & - & 56 & - & 37 & - \\
\hline
\end{tabular}

Table 5.3: Separation characteristics predicted by CFD and measured in experiments at the TUD. FS $=0.30, \operatorname{Re}_{\mathrm{D}}=0.86 \times 10^{5}, \mathrm{Sw}=6.61, d / D=1.00 \times 10^{-3}, \alpha_{\text {inlet }}=0.25$.

case the hold up is 0.42 , much higher than the inlet oil volume fraction of $\alpha_{\text {inlet }}=0.25$. Also, the maximum oil volume fraction that is reached in the center is just over 0.80 . This is due to the high mixture viscosity resulting from the use of the Ishii-Zuber drag law. At these high oil volume fractions the drag is so high that further separation is not possible within the residence time of the fluids inside the separator.

The time-averaged radial distribution of the oil volume fraction at $z=0.50,1.00$ and $1.50 \mathrm{~m}$ is shown in figure 5.15. In the center the oil volume fraction increases until it reaches the maximum of $\alpha=0.82$. The oil-rich core becomes more narrow in downstream direction. Note that the annular reversed flow region is still present at $z=1.00 \mathrm{~m}$, so the outer part of the oil rich core is transported upstream.

As the oil phase in the outer region moves inwards, part of it is captured by the annular reversed flow region. The iso-surface with zero axial velocity, marking the boundaries of the annular reversed flow region, is not a stream tube so fluid is moving in and out of this region. Two effects that are seen are the oil phase moving inward due to the centrifugal force and in and out flow due to the unsteadiness of the flow. The latter effect can be averaged out by examining the time-averaged flow to study the flow of oil through the separator. The annular region of reversed flow does not appear to be beneficial to the separation, since analysis shows that most of the oil, that is collected in that region, is recirculated back into the outer region. Near the ISE the annular reversed flow region has a positive time-averaged radial oil velocity. This is to be expected since the capacity to transport oil through the center is limited due to the low axial velocity and small diameter of that part of the core which has a positive axial velocity. Therefore a relatively large fraction of the oil needs to separate in the downstream part of the separator where the annular reversed flow region is small or has ended.

To determine the separation performance the separation efficiencies $\eta_{1}$ and $\eta_{2}$ are used

$$
\begin{aligned}
& \eta_{1}=1-\frac{Q_{o, H P O}}{Q_{o, \text { inlet }}} \\
& \eta_{2}=1-\frac{1}{1-F S} \frac{Q_{o, H P O}}{Q_{o, \text { inlet }}}
\end{aligned}
$$

The separation characteristics for the nominal two-phase flow case obtained by CFD and by the experiments are given in table 5.3. The numerical simulations show high separation 


\begin{tabular}{|c|c|c|c|c|c|}
\hline & $\alpha_{\text {inlet }}$ & $d / D$ & $\mathrm{FS}$ & $\mathrm{Re}_{\mathrm{D}}$ & $\mathrm{Sw}$ \\
\hline \hline Single-phase flow & - & - & 0.30 & $1.81 \times 10^{5}$ & 6.72 \\
Two-phase flow & 0.25 & $1.00 \times 10^{-3}$ & 0.30 & $0.86 \times 10^{5}$ & 6.61 \\
\hline
\end{tabular}

Table 5.4: Dimensionless variables in single and two-phase flow comparison.

efficiencies, which indicates that the present design functions well as a bulk separator. However, the efficiency measurements at the TUD show a much lower separation efficiency. The difference between experiment and numerical simulation will be discussed in more detail in subsequent sections.

\subsubsection{Comparison of single and two-phase flow}

The solution of the two-phase flow is compared to the solution of the single-phase flow at the same flow rate. The comparison will show the effect on the flow of adding the lighter phase, as predicted using the two-fluid model. The non-dimensional variables used here are listed in table 5.4. The $\mathrm{Re}_{\mathrm{D}}$ differs between the single and two-phase flow cases because of the change in mixture density and mixture viscosity. Also the swirl angle Sw is slightly lower for the two-phase case, however, the difference is smaller than $2 \%$. To investigate whether such a small difference in Sw has a significant impact on the flow, an

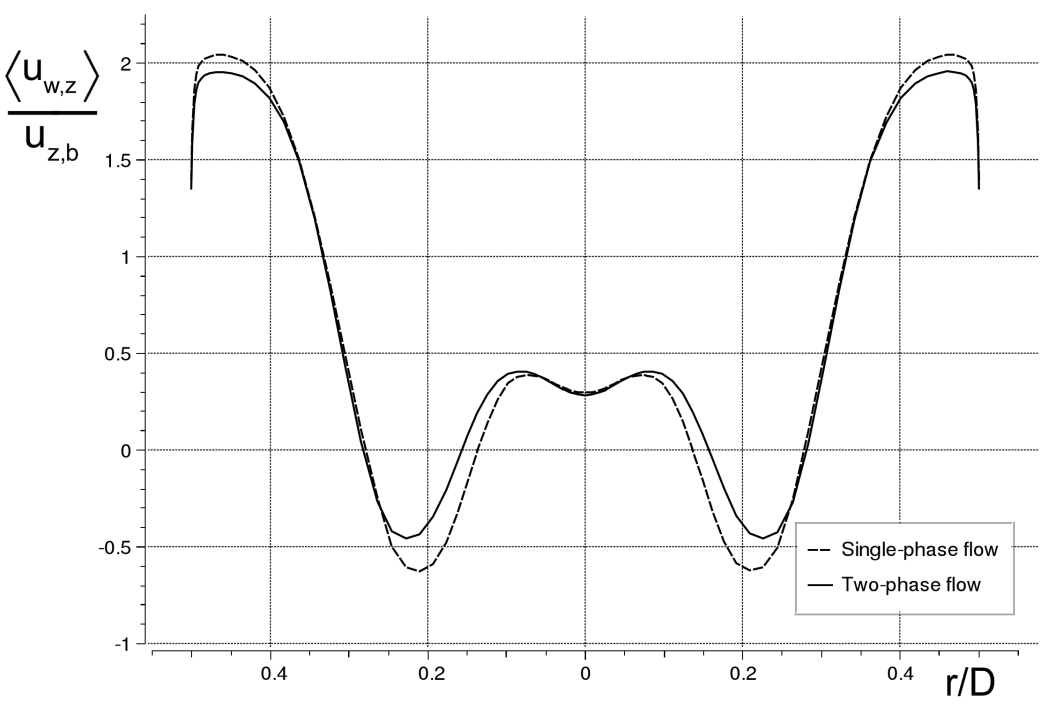

Figure 5.16: Comparison of the radial distribution of the time-averaged axial water velocity on a line through the center of the pipe at $z=0.50 \mathrm{~m}$ for single-phase water flow (dashed) and two-phase flow (solid). Single-phase flow: $\mathrm{FS}=0.30, \mathrm{Re}_{\mathrm{D}}=1.81 \times 10^{5}, \mathrm{Sw}=6.72$; two-phase flow: $\mathrm{FS}=0.30, \operatorname{Re}_{\mathrm{D}}=0.86 \times 10^{5}, \mathrm{Sw}=6.61, d / D=1.00 \times 10^{-3}, \alpha_{\text {inlet }}=0.25$. 
additional simulation for single-phase water flow has been carried out using the vane-less geometry, which is shown in figure 4.36. The flow in this simulation has the same $\operatorname{Re}_{\mathrm{D}}$ as the single-phase flow case: $\operatorname{Re}_{\mathrm{D}}=1.81 \times 10^{5}$ and the swirl angle Sw of the two-phase flow case: $S_{w}=6.61$. The comparison between the single-phase flows with $S_{w}=6.72$ and the one with $\mathrm{Sw}=6.61$ is discussed in appendix $\mathrm{C}$. The conclusion is that for singlephase flow the change in Sw does result in minor changes only. This suggests that the differences the between the single and the two-phase flow case are largely caused by other factors.

Figures 5.16 and 5.17 show the time-averaged radial distribution of the axial and azimuthal water velocities, respectively, on a line through the axis of the pipe at $z=0.50 \mathrm{~m}$ for single-phase water flow and two-phase oil-water flow. In the distribution of the axial velocity the $\mathrm{W}$-shaped flow pattern is seen in both cases. For the two-phase flow, the axial water velocity is equal in the center, less negative in the annular reversed flow region and lower in the outer region. As seen from the distribution of the azimuthal water velocity, the solid-body rotation core has a lower strength for the two-phase flow case.

Figures 5.18(a) and 5.18(b) show the radial distribution of the time-averaged axial and azimuthal water velocities, respectively, on a line through the axis of the pipe at $z=$ $1.50 \mathrm{~m}$ for single-phase water flow and two-phase oil-water flow. For the azimuthal water velocity the difference between the single and the two-phase flow results is similar to the one at the upstream station at $z=0.50 \mathrm{~m}$. However, in the center of the pipe the axial component of the water velocity now differs significantly.

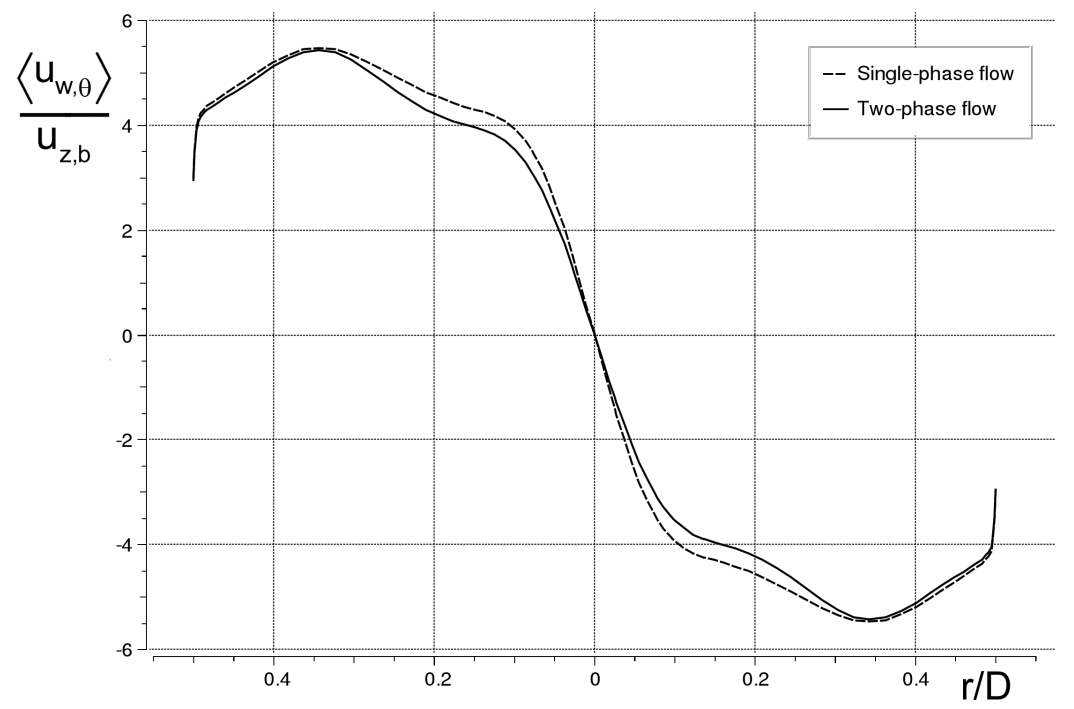

Figure 5.17: Comparison of the radial distribution of the time-averaged azimuthal water velocity on a line through the center of the pipe at $z=0.50 \mathrm{~m}$ for single-phase water flow (dashed) and two-phase flow (solid). Single-phase flow: $\mathrm{FS}=0.30, \mathrm{Re}_{\mathrm{D}}=1.81 \times 10^{5}, \mathrm{Sw}=6.72$; two-phase flow: $\mathrm{FS}=0.30, \operatorname{Re}_{\mathrm{D}}=0.86 \times 10^{5}, \mathrm{Sw}=6.61, d / D=1.00 \times 10^{-3}, \alpha_{\text {inlet }}=0.25$. 


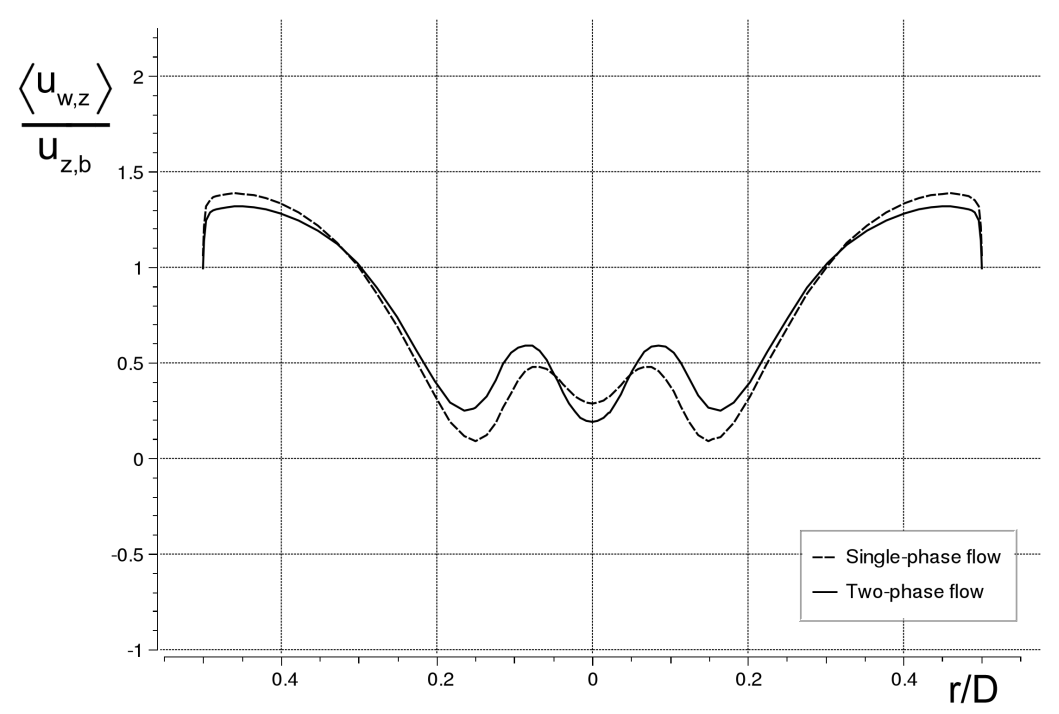

(a) Axial water velocity

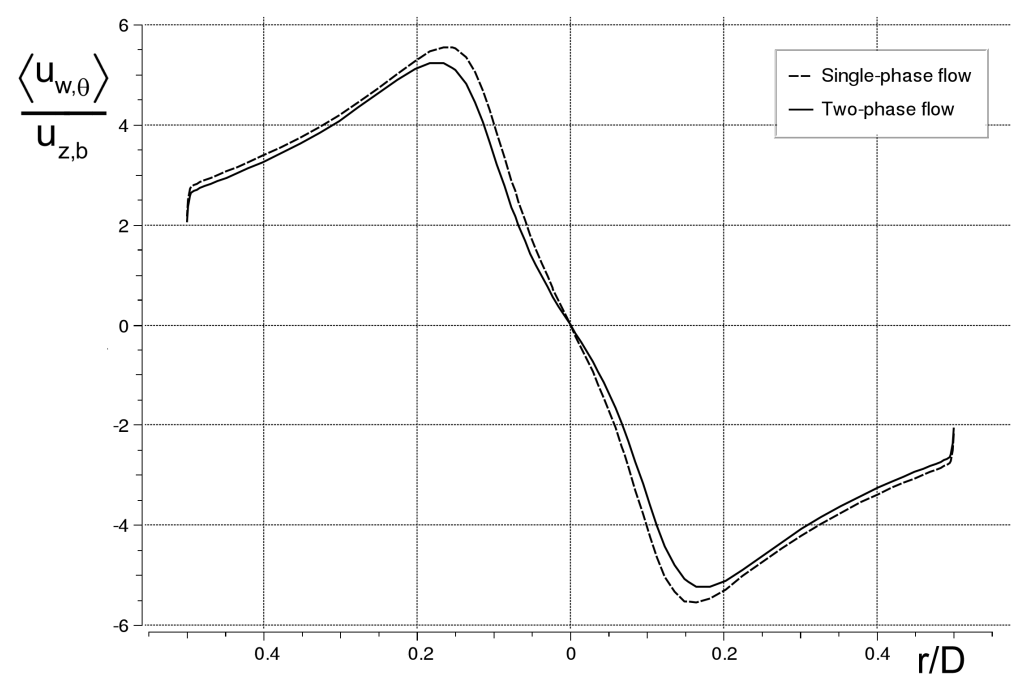

(b) Azimuthal water velocity

Figure 5.18: Comparison of the radial distribution of the time-averaged axial (top) and azimuthal (bottom) water velocity on a line through the center of the pipe in the plane $z=1.50 \mathrm{~m}$ for single-phase water flow (dashed) and two-phase flow (solid). Single-phase flow: $F S=0.30, \operatorname{Re}_{\mathrm{D}}$ $=1.81 \times 10^{5}, \mathrm{Sw}=6.72$; two-phase flow: $\mathrm{FS}=0.30, \mathrm{Re}_{\mathrm{D}}=0.86 \times 10^{5}, \mathrm{Sw}=6.61, d / D=$ $1.00 \times 10^{-3}, \alpha_{\text {inlet }}=0.25$. 
For the two-phase flow $\operatorname{Re}_{\mathrm{D}}=0.86 \times 10^{5}$, which is lower, but close to the values of the Reynolds numbers of the single-phase water flows shown in figures 4.22 and 4.23 . Therefore, similar scaling behavior of the velocity distributions might be expected. This appears to be true for the azimuthal velocity; for lower inlet $\mathrm{Re}_{\mathrm{D}}$ slightly more dissipation is observed yielding a lower azimuthal velocity. Yet for the axial velocity profile, a different trend is seen. The annular reversed flow region is less pronounced for the two-phase flow. This is clearly caused by the segregation of the phases, not the higher viscosity of the oil. Note that even for the pure oil case, with $\operatorname{Re}_{\mathrm{D}}=1.05 \times 10^{4}$, the most negative velocity in the reversed flow region and the width of this region are roughly the same as seen in the single-phase water flows.

As expected for the higher $\operatorname{Re}_{\mathrm{D}}$ for the single-phase flow, the Reynolds stresses are higher for that case, often as much as $25 \%$, compared to the ones in the two-phase flow case. In both cases the Reynolds stresses show higher values near the outer region and somewhat lower values in the center. Furthermore, the Reynolds stresses are increasing in the downstream direction for both flows. Likewise, the turbulent dissipation $\epsilon$ is also higher for the single-phase flow than for the two-phase flow. In the case of two-phase flow the turbulent dissipation stays below $30 \mathrm{~m}^{2} / \mathrm{s}^{3}$, except very close to the wall where it rises steeply. In the single-phase flow the turbulent dissipation is about $5 \mathrm{~m}^{2} / \mathrm{s}^{3}$ higher than that for $0<r / D<0.9$ and $z>1.0 \mathrm{~m}$. In the downstream direction little change is seen

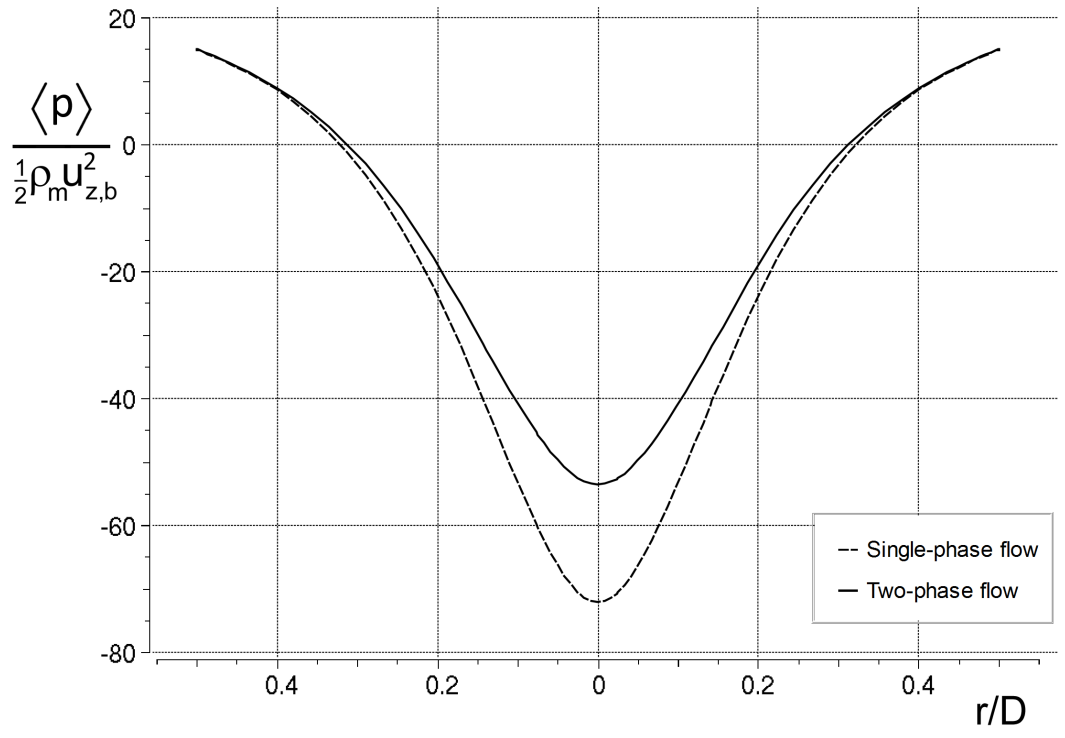

Figure 5.19: Comparison of the radial distribution of the time-averaged pressure on a line through the center of the pipe at $z=1.00 \mathrm{~m}$ for single-phase water flow (dashed) and two-phase flow (solid). Mixture density at the inlet is used for scaling. Single-phase flow: $F S=0.30, \operatorname{Re}_{\mathrm{D}}$ $=1.81 \times 10^{5}, \mathrm{Sw}=6.72$; two-phase flow: $\mathrm{FS}=0.30, \operatorname{Re}_{\mathrm{D}}=0.86 \times 10^{5}, \mathrm{Sw}=6.61, d / D=$ $1.00 \times 10^{-3}, \alpha_{\text {inlet }}=0.25$. 


\begin{tabular}{|l|c|c|c||c|c|c|}
\hline & $\begin{array}{c}\rho \\
\left(\mathrm{kg} / \mathrm{m}^{3}\right)\end{array}$ & $\begin{array}{c}\mu \\
(\mathrm{kg} /(\mathrm{m} . \mathrm{s}))\end{array}$ & $\begin{array}{c}u_{z, b} \\
(\mathrm{~m} / \mathrm{s})\end{array}$ & $\mathrm{FS}$ & $\mathrm{Re}_{\mathrm{D}}$ & $\mathrm{Sw}$ \\
\hline \hline $\mathrm{Q}=75 \%$, water & 1067.8 & $1.183 \times 10^{-3}$ & 1.50 & 0.30 & $1.35 \times 10^{5}$ & 6.71 \\
$\mathrm{Q}=100 \%$, oil & 869 & $16.5 \times 10^{-3}$ & 2.00 & 0.30 & $1.05 \times 10^{4}$ & 6.58 \\
$\mathrm{Q}=100 \%$, oil-water & 1021.1 (inlet) & $2.369 \times 10^{-3}$ & 2.00 & 0.30 & $0.86 \times 10^{5}$ & 6.61 \\
$\mathrm{Q}=100 \%$, water & 1067.8 & $1.183 \times 10^{-3}$ & 2.00 & 0.30 & $1.81 \times 10^{5}$ & 6.72 \\
$\mathrm{Q}=125 \%$, water & 1067.8 & $1.183 \times 10^{-3}$ & 2.50 & 0.30 & $2.26 \times 10^{5}$ & 6.73 \\
\hline
\end{tabular}

Table 5.5: Material properties, flow conditions and dimensionless variables in flow rate variation study.

in the turbulent dissipation rate for both single and two-phase flow.

The radial distribution of the pressure is presented in figure 5.19. Also for two-phase flow, the hydrostatic pressure component is subtracted from the pressure presented here. To calculate the hydrostatic pressure CFX does not use the local mixture density but a constant reference density, here set equal to the density of the water: $\rho_{w}=1067.8 \mathrm{~kg} / \mathrm{m}^{3}$. So the hydrostatic pressure only depends on the height and not on other parameters that have an effect on the mixture density in the separator, such as the oil volume fraction at the inlet or the flow split. In addition, the hydrostatic pressure at $z=1.00 \mathrm{~m}$ is a constant, which magnitude is also arbitrary since the reference height $h$ can be chosen freely. Now,

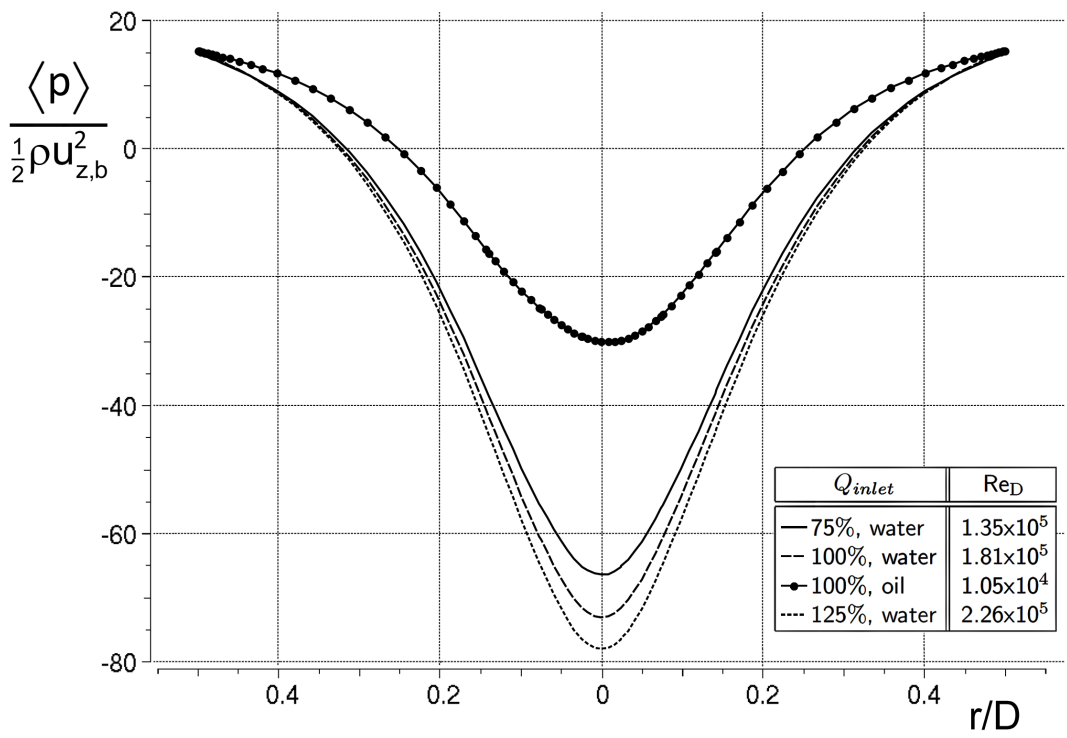

Figure 5.20: Time-averaged radial distribution of non-dimensional pressure on a line through the center of the pipe at $z=1.00 \mathrm{~m}$ for single-phase water flow. Flow rates specified in table 5.5. 
the gradient of the hydrostatic pressure $\rho_{w} g(h-z)$ in the momentum equations of the oil phase does not cancel out with the gravity term $\rho_{o} g$ but forms a constant buoyancy term $\Delta \rho g$ and therefore the hydrostatic pressure will have an effect on the velocity distribution. For comparison, figure 5.20 presents the pressure distribution of the single-phase flows for various values of $R e_{D}$. The single-phase water flow in figure 5.19 corresponds to the case of $100 \%$ flow rate in figure 5.20 . Just as the azimuthal velocity distribution, the pressure distribution of the two-phase flow follows the trend seen in the results for single-phase flow. The pressure in the center is less negative for lower $\operatorname{Re}_{\mathrm{D}}$.

An additional difference between single and two-phase flow is the radial variation of the mixture density. In the two-phase flow case, the mixture density in the center of the pipe can be up to about $10 \%$ lower than at the inlet. Therefore, the pressure will increase less steeply in radial direction in the center for the two-phase flow case. Near the wall the opposite effect is seen since there the mixture density is higher than at the inlet. However, this effect is smaller near the pipe wall since the difference in mixture density between the pipe wall and the inlet is smaller compared to the one between center of the pipe and the inlet. At the wall the pressure for single and two-phase flow are equal due to the boundary condition at the HPO where the pressure is set to the reference value of $0 \mathrm{~Pa}$. The evolution of the pressure with the axial direction show similar trends for both single and the two-phase flow.

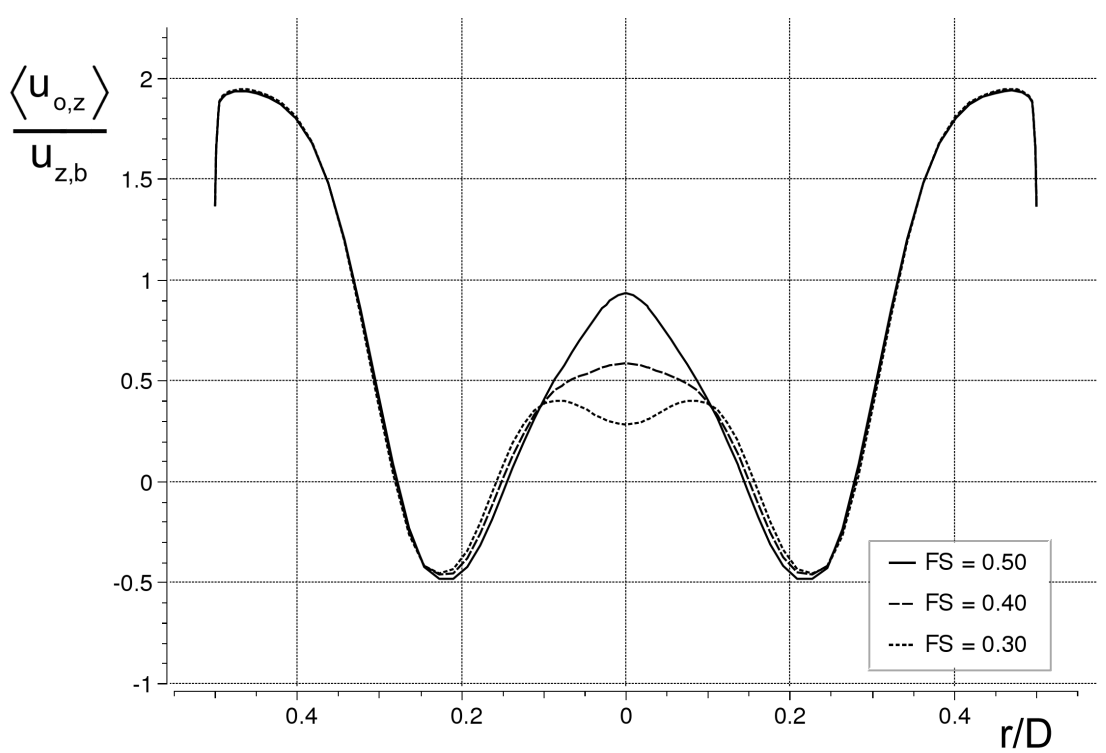

Figure 5.21: Radial distribution of the time-averaged axial oil velocity on a line through the center of the pipe in the plane $z=0.50 \mathrm{~m}$ for flow split $\mathrm{FS}=[0.30,0.40,0.50]$. For all cases $\operatorname{Re}_{\mathrm{D}}=0.86 \times 10^{5}, \mathrm{Sw}=6.61, d / D=1.00 \times 10^{-3}$ and $\alpha_{\text {inlet }}=0.25$. 


\subsection{Separation efficiency studies}

The flow split FS and the inlet oil volume fraction $\alpha_{\text {inlet }}$ have been varied in order to study their effect on the separation efficiency. The results from these numerical simulations are discussed and are subsequently compared to experimental measurements carried out at the TUD. The predictions of the numerical simulations are evaluated and explanations for the differences between numerical and experimental results are given.

\subsubsection{Variation of the flow split}

An increase of separation efficiency can be obtained by increasing the flow split FS. However, after a certain threshold value of FS the separation efficiency hardly improves with further increases in FS, while the pressure drop, as well as the amount of water phase that is leaving through the LPO, will still increase. Often the oil volume fraction in the HPO is required to fall below a certain value, which for example can be specified by legislation. Therefore, an optimum FS can be found for which the separator delivers water with the oil volume fraction below the specified value through the HPO with a minimal pressure drop over the separator.

In the present study the separation efficiency is examined for an inlet oil volume fraction $\alpha_{\text {inlet }}=0.25$ for $\mathrm{FS}=[0.30,0.40$ and 0.50$]$. In two-phase flow the distribution of the velocity for different values of the flow split FS is similar to the ones found in single-phase

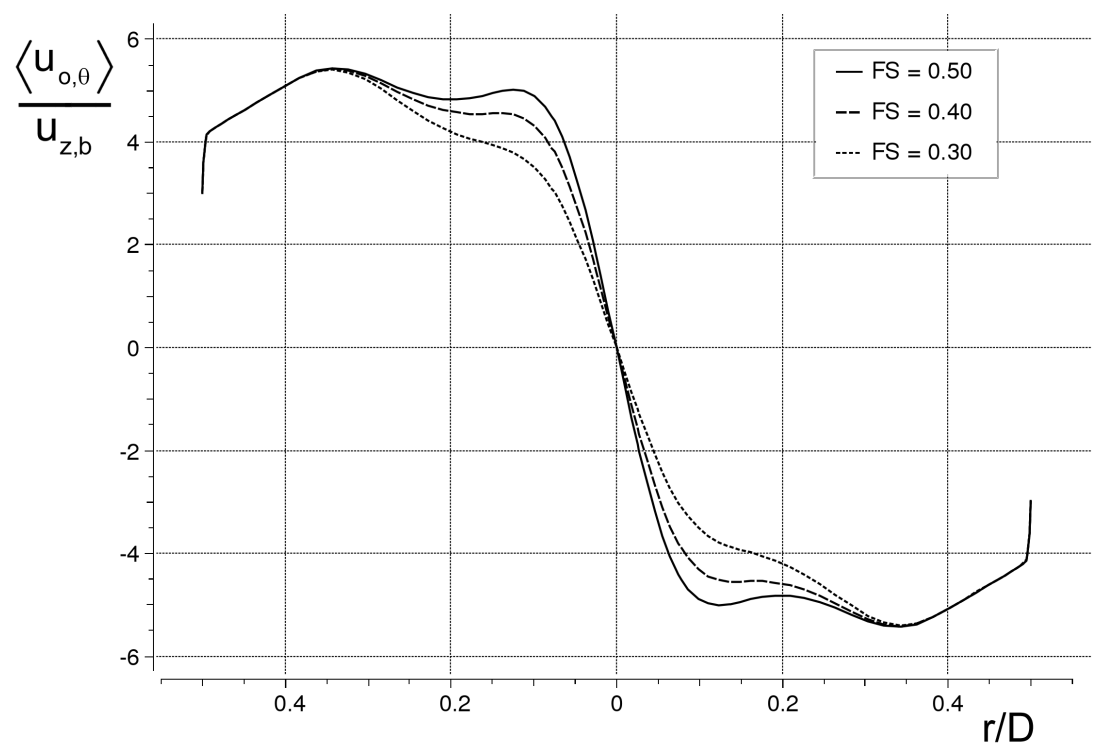

Figure 5.22: Radial distribution of the time-averaged azimuthal oil velocity on a line through the center of the pipe in the plane $z=0.50 \mathrm{~m}$ for flow split $\mathrm{FS}=[0.30,0.40,0.50]$. For all cases $\operatorname{Re}_{\mathrm{D}}=0.86 \times 10^{5}, \mathrm{Sw}=6.61, d / D=1.00 \times 10^{-3}$ and $\alpha_{\text {inlet }}=0.25$. 
flow. The distribution of the time-averaged axial oil velocity on a line through the center of the pipe at $z=0.50 \mathrm{~m}$ for a flow split FS of $0.30,0.40,0.50$ is shown in figure 5.21. Here only the axial velocity of the oil is shown, the axial water velocity is nearly identical except in the boundary layer at the pipe wall. As is seen for single-phase flow, the axial velocity component in the two-phase flow case shows a strong increase in the center of the pipe when the flow split FS is increased. However, the two-phase flow shows a lower axial velocity in the outer region near the pipe wall and less negative axial velocities in the region of reversed flow compared to single-phase flow.

The distribution of the time-averaged azimuthal velocity is shown in figure 5.22. The spin-up of the azimuthal velocity component with increasing FS can clearly be seen. As observed before in figure 5.17, the maximum azimuthal velocity is somewhat lower for two-phase flow compared to single-phase flow, see the single-phase flow results in figure 4.29. Due to the similarity between the velocity distributions of single and two-phase flow for variations in the flow split, only the results in the cross-flow plane $z=0.50 \mathrm{~m}$ are given here.

The radial distribution of the time-averaged pressure on a line through the center of the pipe in the plane $z=1.00 \mathrm{~m}$ for flow split FS $=[0.30,0.40,0.50]$ is shown in figure 5.23. Here larger differences between the results for single and two-phase flow as a function of FS can be seen. Near the pipe wall the pressure is the same for identical flow split for single and two-phase flow. However, a much higher pressure is seen at the axis of the pipe for two-phase flow. The difference in non-dimensional pressure is about 20, or 20 to $30 \%$. The main reason for this difference in the distribution of the pressure is the lower

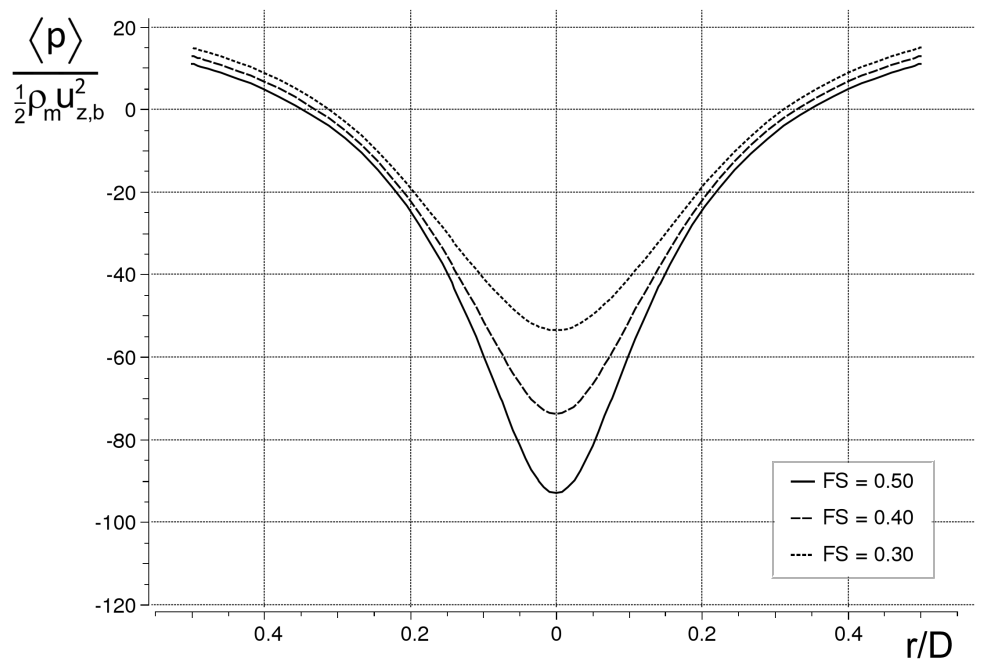

Figure 5.23: Radial distribution of the time-averaged pressure on a line through the center of the pipe in the plane $z=1.00 \mathrm{~m}$ for flow split $\mathrm{FS}=[0.30,0.40,0.50]$. For all cases $\operatorname{Re}_{\mathrm{D}}=$ $0.86 \times 10^{5}, \mathrm{Sw}=6.61, d / D=1.00 \times 10^{-3}$ and $\alpha_{\text {inlet }}=0.25$. 
azimuthal velocity component near the center of the pipe for the two-phase flow cases, which in turn can be related to their lower $\operatorname{Re}_{\mathrm{D}}$.

The non-dimensional pressure drop Eu

$$
\mathrm{Eu}=\frac{\Delta\langle\bar{p}\rangle}{\frac{1}{2} \rho u_{z, b}^{2}}
$$

as function of the flow split FS is shown in figure 5.24. Here $\Delta\langle\bar{p}\rangle$ is the pressure difference between inlet and LPO. Based on the results of the variation in flow split FS for singlephase flow, a power law curve fit is used here. A good fit for this range of FS is obtained by

$$
\mathrm{Eu}=140 \mathrm{FS}^{0.215}
$$

This is reasonably similar to the curve fit obtained for single-phase flow given in equation (4.16). However, due to the lower swirl angle $S w$ and lower Reynolds number $\operatorname{Re}_{\mathrm{D}}$ for equal FS, the non-dimensional pressure drop $\mathrm{Eu}$ is lower in the present two-phase flow case.

The radial distribution of the oil volume fraction on a line through the center in the crossflow plane $z=1.50 \mathrm{~m}$ is shown in figure 5.25 . The oil-rich core becomes narrower as the flow split increases, due to the higher g-force experienced for larger FS. In the center of the pipe, the oil volume fraction increases slightly with increasing FS to a value around $\alpha=0.82$. However, because of the very high mixture viscosity for $\alpha>0.80$ a further increase in oil volume fraction is not observed when the flow split is increased from 0.40 to 0.50 . The oil volume fraction is even slightly lower for $F S=0.50$. This may be explained by the higher axial velocity for $F S=0.50$. The higher axial velocity shortens the residence time for the oil in the central core inside the separator, giving the oil phase less time to

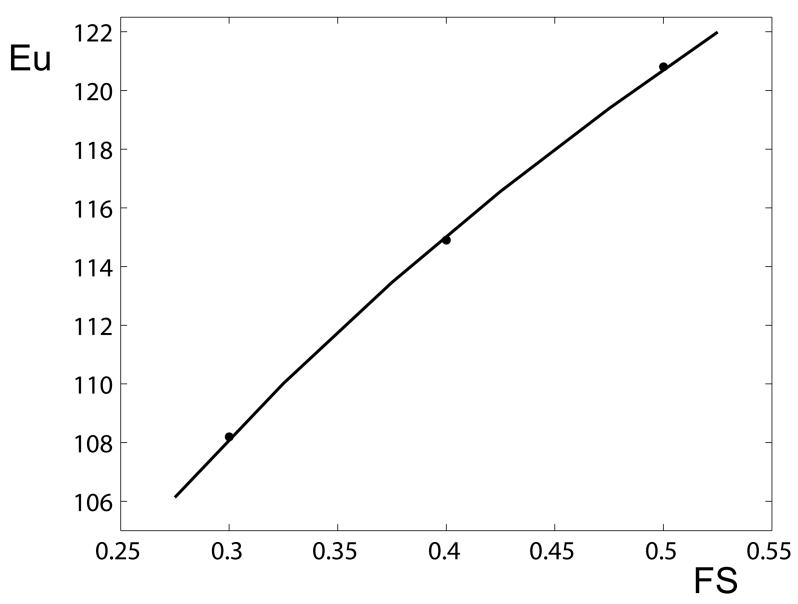

Figure 5.24: Non-dimensional pressure drop Eu as function of the flow split. The power law fit $\mathrm{Eu}=140 \mathrm{FS}^{0.215}$ is used. For all cases $\operatorname{Re}_{\mathrm{D}}=0.86 \times 10^{5}, \mathrm{Sw}=6.61, d / D=1.00 \times 10^{-3}$ and $\alpha_{\text {inlet }}=0.25$. 


\begin{tabular}{|c|c|c|c|c|c|c|c|}
\hline FS & $\alpha_{H P O}$ & $\alpha_{L P O}$ & hold up & $\eta_{1}$ & $\frac{\Delta Q_{o}}{Q_{o, \text { inlet }}}$ & $\eta_{2}$ & $\frac{1}{1-F S} \frac{\Delta Q_{o}}{Q_{o, \text { inlet }}}$ \\
& $(\%)$ & $(\%)$ & $(\%)$ & $(\%)$ & $(\%)$ & $(\%)$ & $(\%)$ \\
\hline \hline 0.30 & 4.8 & 72 & 42 & 86 & 0.56 & 80 & 0.80 \\
0.40 & 0.15 & 63 & 40 & 99.7 & 0.25 & 99.4 & 0.41 \\
0.50 & 0.08 & 50 & 38 & 99.9 & 0.46 & 99.7 & 0.91 \\
\hline
\end{tabular}

Table 5.6: Separation characteristics as function of flow split for two-phase flow. $\operatorname{Re}_{\mathrm{D}}=$ $0.86 \times 10^{5}, \mathrm{Sw}=6.61, d / D=1.00 \times 10^{-3}$ and $\alpha_{\text {inlet }}=0.25$.

build up in the center.

The separation characteristics as function of the flow split are summarized in table 5.6. As expected both measures of separation efficiency $\eta_{1}$ and $\eta_{2}$ increase for higher FS. However, for $\mathrm{FS}=0.40$, the efficiencies are already above $99 \%$, so little increase in separation efficiency can be gained by further increasing FS. From table 5.6 it can also be seen that the hold up of oil decreases with increasing flow split.

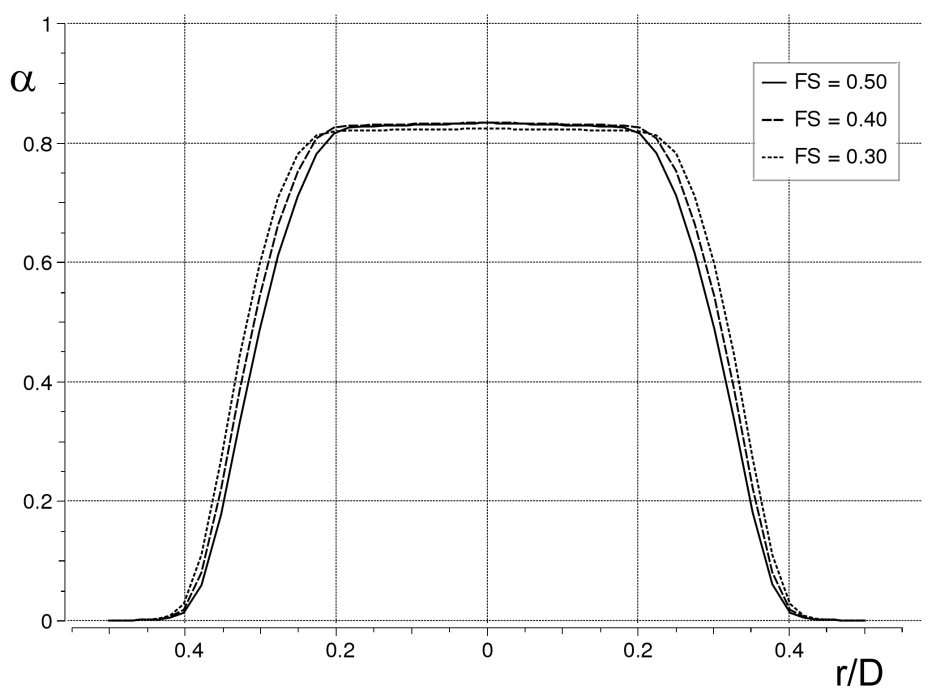

Figure 5.25: Radial distribution of the time-averaged oil volume fraction $\alpha$ on a line through the center in the plane $z=1.50 \mathrm{~m}$ for $\mathrm{FS}=[0.30,0.40,0.50]$. For all cases $\operatorname{Re}_{\mathrm{D}}=0.86 \times 10^{5}$, $\mathrm{Sw}=6.61, d / D=1.00 \times 10^{-3}$ and $\alpha_{\text {inlet }}=0.25$. 


\begin{tabular}{|c|c|c|c|c|c||c|}
\hline$\alpha_{\text {inlet }}$ & $\mathrm{FS} / \alpha_{\text {inlet }}$ & $d / D$ & $\mathrm{FS}$ & $\mathrm{Re}_{\mathrm{D}}$ & $\mathrm{Sw}$ & $\mathrm{Eu}$ \\
\hline \hline 0.15 & 1.2 & $1.00 \times 10^{-3}$ & 0.18 & $1.19 \times 10^{5}$ & 6.62 & 100.9 \\
0.25 & 1.2 & $1.00 \times 10^{-3}$ & 0.30 & $0.86 \times 10^{5}$ & 6.61 & 108.7 \\
0.40 & 1.2 & $1.00 \times 10^{-3}$ & 0.48 & $0.49 \times 10^{5}$ & 6.61 & 118.2 \\
\hline
\end{tabular}

Table 5.7: Dimensionless variables in study of the effect of inlet oil volume fraction.

\subsubsection{Variation of the inlet oil volume fraction}

To further investigate the performance of the separator a study has been carried out into the effect on the separation efficiency of the oil fraction at the inlet $\alpha_{\text {inlet }}=[0.15,0.25$, 0.40 ]. The flow split FS will be varied accordingly. For each numerical simulation the ratio $\mathrm{FS} / \alpha_{\text {inlet }}$ is set equal to 1.2 . The non-dimensional parameters used in this study are given in table 5.7 .

The trends observed in the distribution of the velocity for varying $\alpha_{\text {inlet }}$ are very similar to the results presented in section 5.4.1 and therefore the distribution of the velocity will not be shown here in detail. The increase is oil content at the inlet is of relatively small influence on the velocity distribution, although some decrease in swirl is seen due to the lower $\mathrm{Re}_{\mathrm{D}}$ in the cases with higher inlet oil volume fractions. The decrease in swirl leads to a slightly higher pressure in the center of the pipe for the flows with higher inlet oil
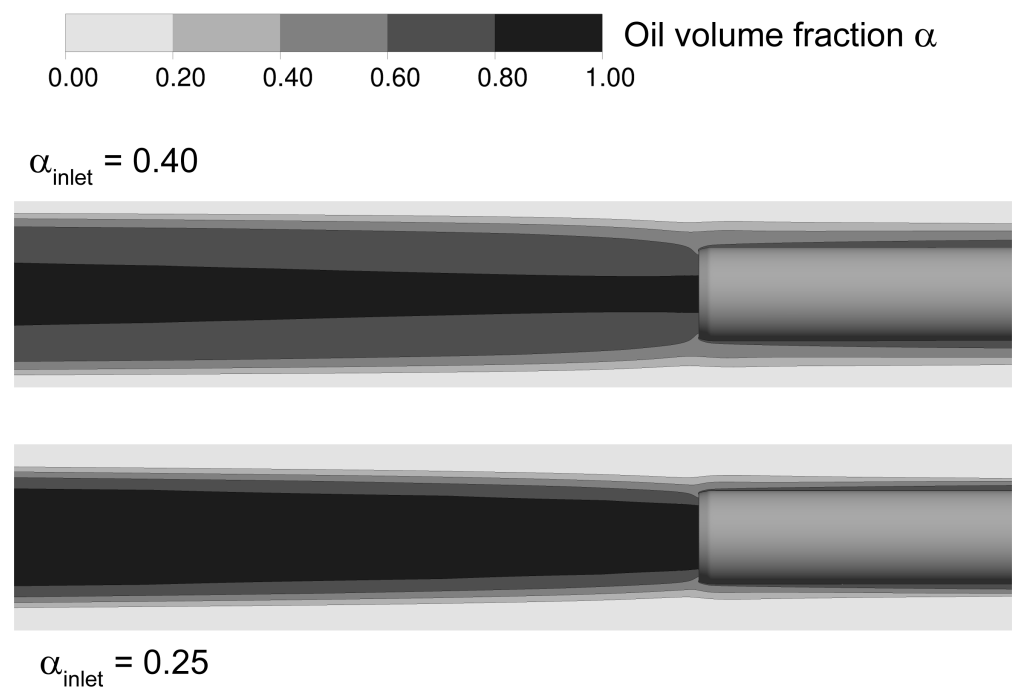

Figure 5.26: Distribution of the time-averaged oil volume fraction $\alpha$ on a plane through the axis of the separator in the region near the pick-up tube for $\alpha_{\text {inlet }}=0.40$ (top) and $\alpha_{\text {inlet }}=0.25$ (bottom). Spill-over of oil can clearly be seen for $\alpha_{\text {inlet }}=0.40$. Non-dimensional parameters are given in table 5.7 . 
volume fractions compared to the results of section 5.4.1. However, the variation of the pressure distribution with changing FS is very similar.

The separation performance for the present study is given in table 5.8. The efficiencies $\eta_{1}$ and $\eta_{2}$ rise initially when the inlet oil volume fraction is increased from 0.15 to 0.25 and the FS is adjusted from 0.18 to 0.30 , although the difference in efficiency is small. This increase in efficiency may be attributed to the higher centrifugal forces that are generated for $\mathrm{FS}=0.30$. However, for the case of $\alpha_{\text {inlet }}=0.40$ the efficiencies decrease. This is especially clear for $\eta_{2}$, in which the quality of the water outlet is incorporated, see equation (3.33). The numerical simulations predict that the separator is flooded with oil for these high inlet oil volume fractions and at the pick-up tube this leads to large spill-overs of oil towards to HPO. The spill-over of oil is shown in figure 5.26, which shows the distribution of the oil volume fraction $\alpha$ on a plane through the axis of the separator in the region near the pick-up tube for $\alpha_{\text {inlet }}=0.40$ and $\alpha_{\text {inlet }}=0.25$. This flooding results in a very high oil volume fraction at the HPO. Apparently, the even higher g-forces seen for FS = 0.48 cannot compensate for the higher mixture viscosity experienced by the oil phase as it moves towards the center. For example, compared to the case of $\alpha_{\text {inlet }}=0.15$, the mixture viscosity at the inlet increases by $130 \%$ and $225 \%$ for $\alpha_{\text {inlet }}=0.25$ and $\alpha_{\text {inlet }}=$ 0.40 , respectively. This higher mixture viscosity slows down the inward motion of the oil phase, which results in a wider band of oil-rich fluid as is shown in figure 5.27. This figure shows the radial distribution of the oil volume fraction $\alpha$ on a line through the center at $z=1.50 \mathrm{~m}$ for $\alpha_{\text {inlet }}=[0.15,0.25,0.40]$. The higher mixture viscosity decreases the

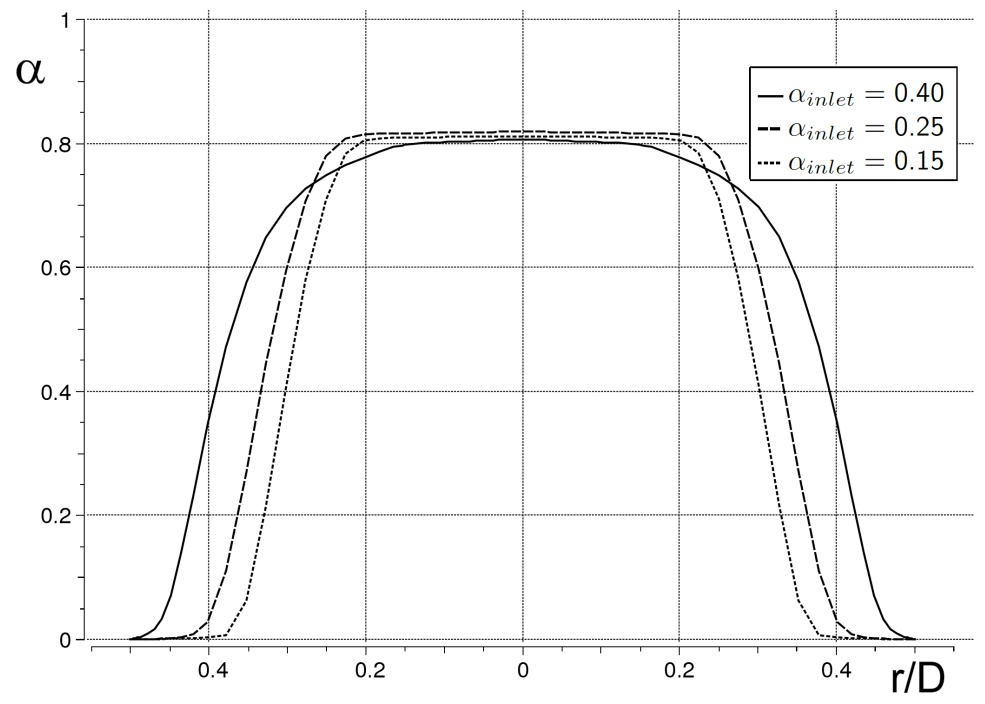

Figure 5.27: Radial distribution of the time-averaged oil volume fraction $\alpha$ on a line through the center in the plane $z=1.50 \mathrm{~m}$ for $\alpha_{\text {inlet }}=[0.15,0.25,0.40]$. Non-dimensional parameters are given in table 5.7 . 


\begin{tabular}{|c|c|c|c|c|c|c|c|}
\hline$\alpha_{\text {inlet }}$ & $\alpha_{H P O}$ & $\alpha_{L P O}$ & hold up & $\eta_{1}$ & $\frac{\Delta Q_{o}}{Q_{o, \text { inlet }}}$ & $\eta_{2}$ & $\frac{1}{1-F S} \frac{\Delta Q_{o}}{Q_{o, \text { inlet }}}$ \\
& $(\%)$ & $(\%)$ & $(\%)$ & $(\%)$ & $(\%)$ & $(\%)$ \\
\hline \hline 0.15 & 3.1 & 70 & 37 & 83 & 0.44 & 79 & 0.54 \\
0.25 & 4.8 & 72 & 42 & 86 & 0.56 & 80 & 0.80 \\
0.40 & 13 & 70 & 50 & 83 & 0.05 & 68 & 0.10 \\
\hline
\end{tabular}

Table 5.8: Separation characteristics as function of the inlet oil volume fraction. Non-dimensional parameters are given in table 5.7 .

flow of oil towards the center to such extent that the maximum oil volume fraction in the case of $\alpha_{\text {inlet }}=0.40$ is even lower than for $\alpha_{\text {inlet }}$ is 0.15 and 0.25 . Currently the ratio $\mathrm{FS} / \alpha_{\text {inlet }}$ has been fixed to a constant value, but the results of the simulations indicate that for a better separation performance this ratio should be increased for higher $\alpha_{\text {inlet }}$. However, for increasing FS the centrifugal acceleration increases only in the center of the pipe, while the spin-up effect of the azimuthal velocity component is absent at larger radius, see for instance figure 4.32 . The issues for high $\alpha_{\text {inlet }}$ seen above may therefore persist and the current separator may only be suited for $\alpha_{\text {inlet }}$ below a certain threshold value. Also note that the hold-up of oil increases considerably with increasing inlet oil volume fraction. 


\subsubsection{Validation by comparison with experimental data}

Figures 5.28 and 5.29 show the separation efficiencies $\eta_{1}$ and $\eta_{2}$, respectively, determined by numerical simulations and by measurements as function of flow split FS for $\alpha_{\text {inlet }}$ $=0.25$. The numerical simulation greatly overpredicts the separation efficiency of the separator by as much as $30 \%$ and $60 \%$ for $\eta_{1}$ and $\eta_{2}$, respectively. The results from the numerical simulations indicate that the maximum efficiency is already attained at a FS of about 0.40 . However, the measurements show that both $\eta_{1}$ and $\eta_{2}$ are still increasing with increasing FS for FS $>0.70$. In the experiments an average $\alpha_{\text {inlet }}$ of 0.248 has been determined with a standard deviation of $5.3 \times 10^{-3}$.

The numerical results for $\eta_{1}$ as function of FS for various inlet volume fractions $\alpha_{\text {inlet }}=$ [ $0.15,0.25,0.40]$ are compared with experimental data in figure 5.30. Again the numerical results deviate considerably from the experimental values. The numerical simulations show the highest efficiency for $\alpha_{\text {inlet }}=0.25$, although the change in efficiency is relatively small. The experimental results show that the increase in $\eta_{1}$ for increasing FS seen for $\alpha_{\text {inlet }}=$ 0.25 also holds for a wider range of inlet oil volume fractions. Contrary to the numerical

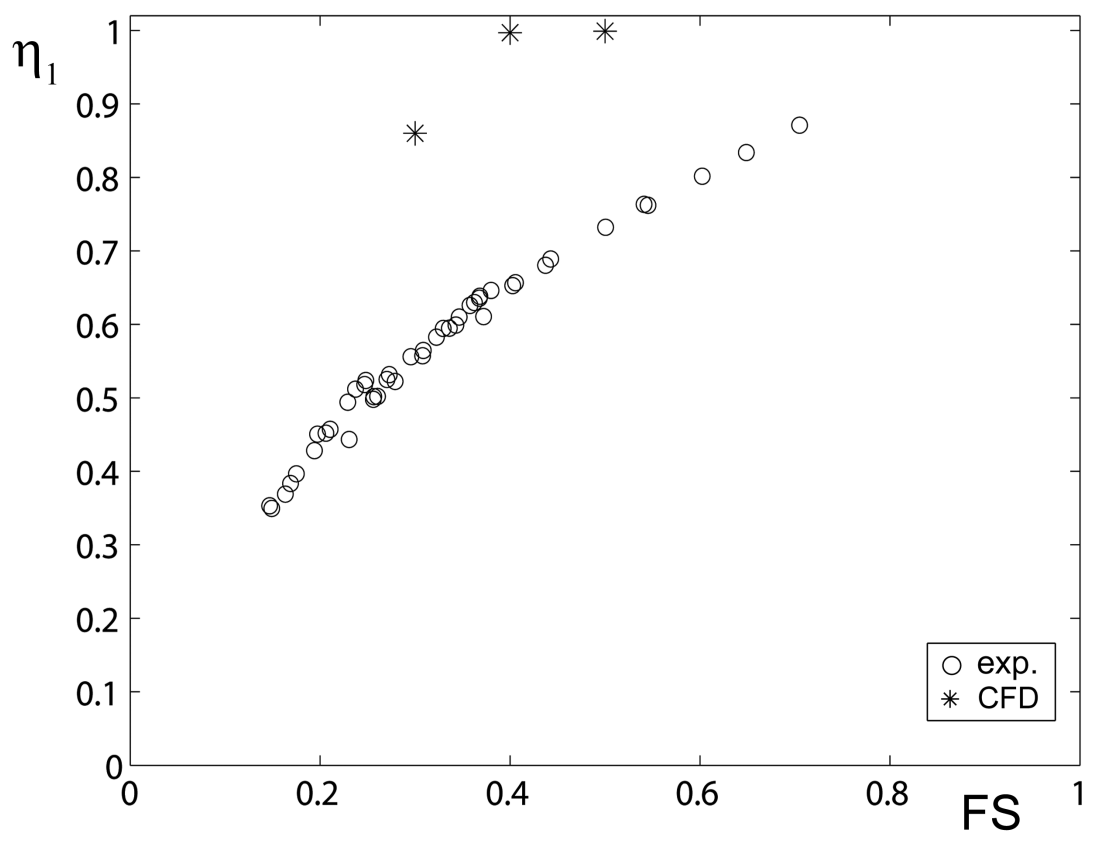

Figure 5.28: Comparison of separation efficiency $\eta_{1}$ determined by numerical simulations $\left({ }^{*}\right)$ and by measurements (o) as function of flow split FS. In numerical simulations: $\alpha_{\text {inlet }}=0.25, \operatorname{Re}_{\mathrm{D}}$ $=0.86 \times 10^{5}, \mathrm{Sw}=6.61$ and $d / D=1.00 \times 10^{-3}$, in measurements: $\alpha_{\text {inlet }}=0.248$. Experimental results by Laurens van Campen, TUD. 
results, a decline in efficiency for $\alpha_{\text {inlet }}=0.40$ is not observed. This indicates that in the experiments the flooding effect is not present.

Since the ratio $\mathrm{FS} / \alpha_{\text {inlet }}$ is much higher for lower inlet oil volume fractions, it is no surprise that $\eta_{1}$ is higher for lower values of $\alpha_{\text {inlet }}$. The numerical and experimental results for $\eta_{1}$ are shown as function of FS $/ \alpha_{\text {inlet }}$ for $\alpha_{\text {inlet }}=[0.15,0.25,0.40]$ in figure 5.31. The experimental results now clearly show that when FS is adjusted to the inlet oil volume fraction, the separator performs better for higher inlet oil volume fractions. This is an opposite trend than the one observed in the numerical simulations.

The measurements may show a higher $\eta_{1}$ as function of $F S / \alpha_{\text {inlet }}$ for higher $\alpha_{\text {inlet }}$ due to the spin-up of the azimuthal velocity component. The pick-up tube occupies about $25 \%$ of the cross-flow plane. For an $\alpha_{\text {inlet }}$ of 0.40 this may appear relatively small and at first glance a larger diameter for the pick-up tube should be considered in the design. However, a relatively small diameter pick-up tube will lead to a stronger inward motion and so to more spin-up of the azimuthal velocity component. Further investigation with different pick-up tube designs is needed, but it appears that a relatively small pick-up tube is beneficial to the separation. Unfortunately, this will also increase the pressure

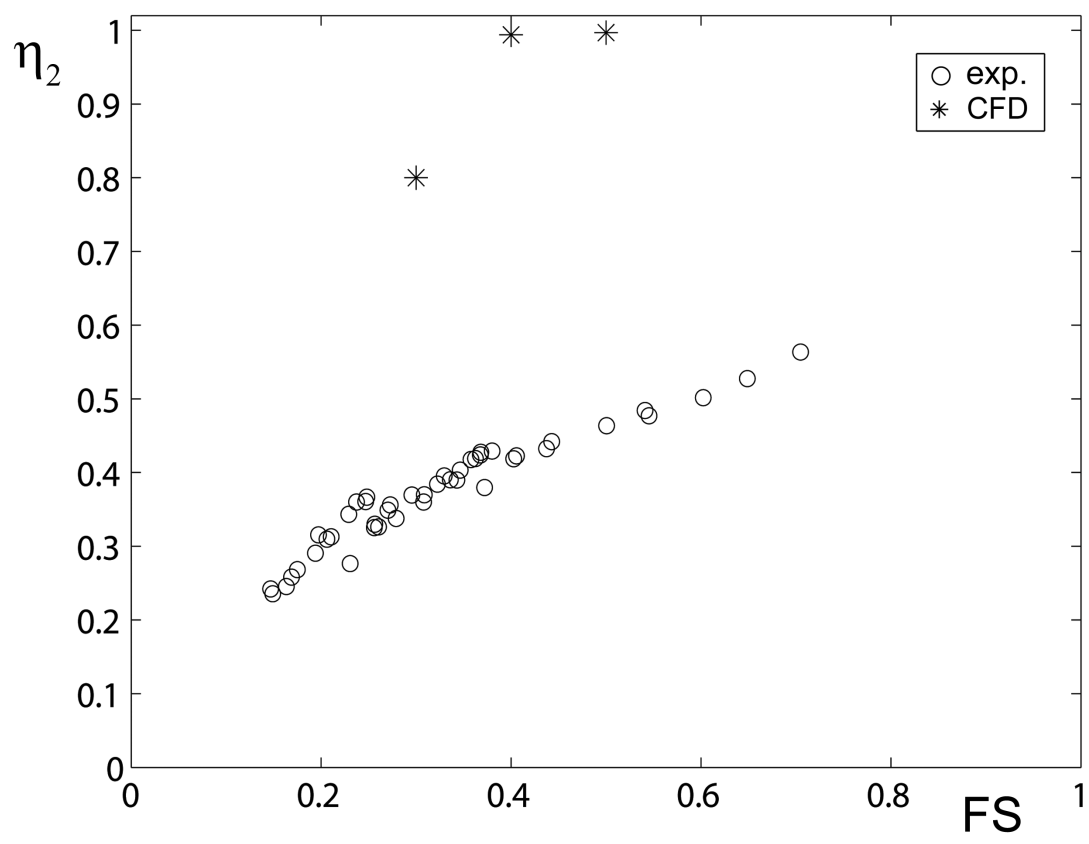

Figure 5.29: Comparison of separation efficiency $\eta_{2}$ from numerical simulations $\left({ }^{*}\right)$ and from measurements (o) as function of flow split FS. In numerical simulations: $\alpha_{\text {inlet }}=0.25, \operatorname{Re}_{\mathrm{D}}=$ $0.86 \times 10^{5}, \mathrm{Sw}=6.61$ and $d / D=1.00 \times 10^{-3}$, in measurements: $\alpha_{\text {inlet }}=0.248$. Experimental results by Laurens van Campen, TUD. 
drop between inlet and LPO. A compromise between these two conflicting requirements has to be found. This compromise will depend on the specific conditions of the oil field from which the separator receives its oil-water mixture. For example, these conditions can be the (reservoir) pressure that is available, the cost of re-pressurizing the flow downstream of the separator by using booster pumps and the difficulty of meeting separation efficiency targets.

Figure 5.32 shows $\eta_{2}$ as function of the flow split for the numerical results and experimental data for different values of the inlet volume fraction $\alpha_{\text {inlet }}=[0.15,0.25,0.40]$. As before, the numerical simulation predicts separation efficiencies which are much higher than the ones seen in the measurements. The difference between numerical and experimental results for $\eta_{2}$ is even larger than for $\eta_{1}$. In the simulations an increase in $\eta_{2}$ is seen for $\alpha_{\text {inlet }}=0.25$ compared to the $\alpha_{\text {inlet }}=0.15$ case, while the experiments clearly show lower $\eta_{2}$ for increasing $\alpha_{\text {inlet }}$. Also, the experimental data shows a larger difference in separation efficiency $\eta_{2}$ for different inlet volume fractions compared to the results for $\eta_{1}$ in figure 5.30. However, as is shown in figure 5.33, if the flow split is scaled by the inlet oil volume fraction the experimental results more or less collapse into a single curve within

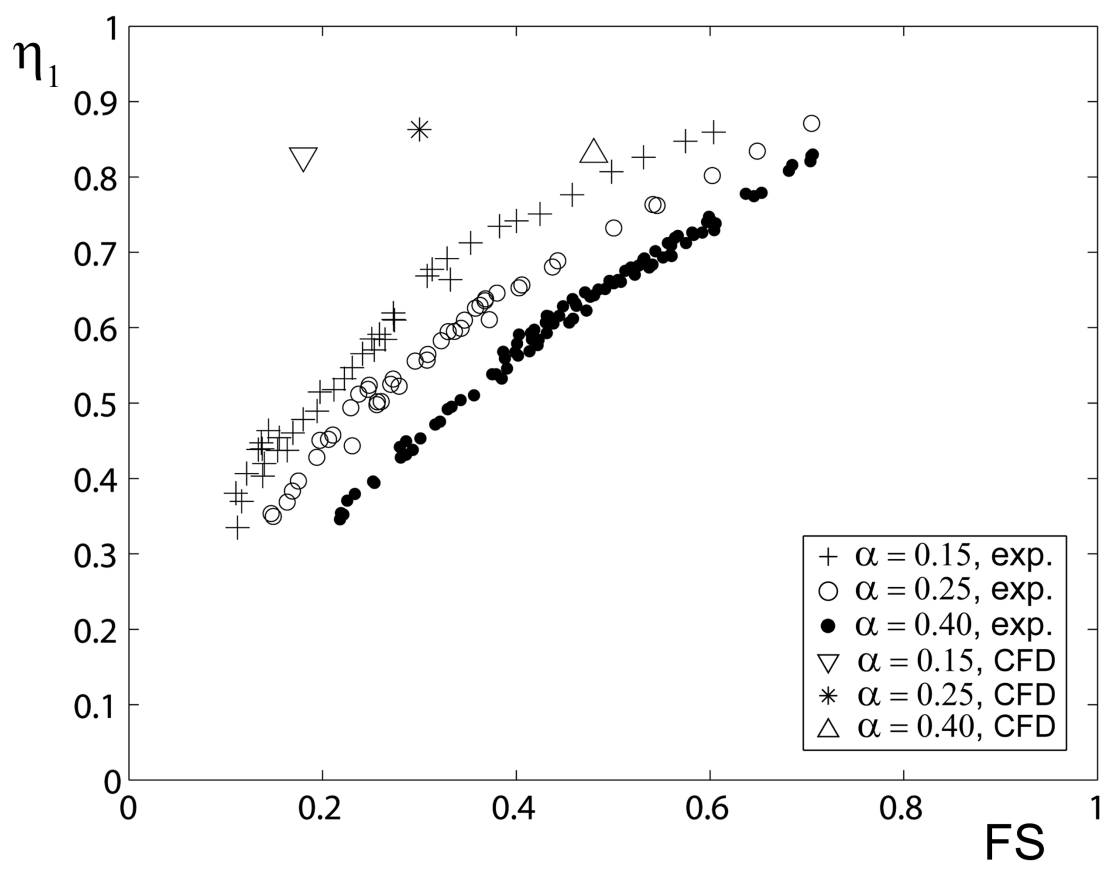

Figure 5.30: Comparison of separation efficiency $\eta_{1}$ as function of FS determined from numerical simulations and from measurements for $\alpha_{\text {inlet }}=[0.15,0.25,0.40]$. Non-dimensional parameters used in numerical simulations are given in table 5.7. Experimental results by Laurens van Campen, TUD. 
the variation of $\eta_{2}$ for repeated measurement at equal flow split. The scaling of FS with $\alpha_{\text {inlet }}$ points out that the decline in separation efficiency $\eta_{2}$ for high $\alpha_{\text {inlet }}$ is not present in the experimental data. The same trend was seen before for $\eta_{1}$ as function of FS.

The large difference between the numerical and experimental results may, at least partially, be explained in terms of three issues:

- the formulation of the drag between the phases.

- the inability of the present numerical simulations to capture directly the effect of turbulence on the motion of the oil phase.

- the mismatch between the droplet size used in the numerical simulations and the droplet size distribution present in the separator in the experimental setup at the TUD.

Not only is the efficiency overpredicted by the numerical simulations, also the trend with increasing inlet oil volume fraction $\alpha_{\text {inlet }}$ is different. The difference may be explained by droplet coalescence. Coalescence is expected to occur more often for high inlet oil volume

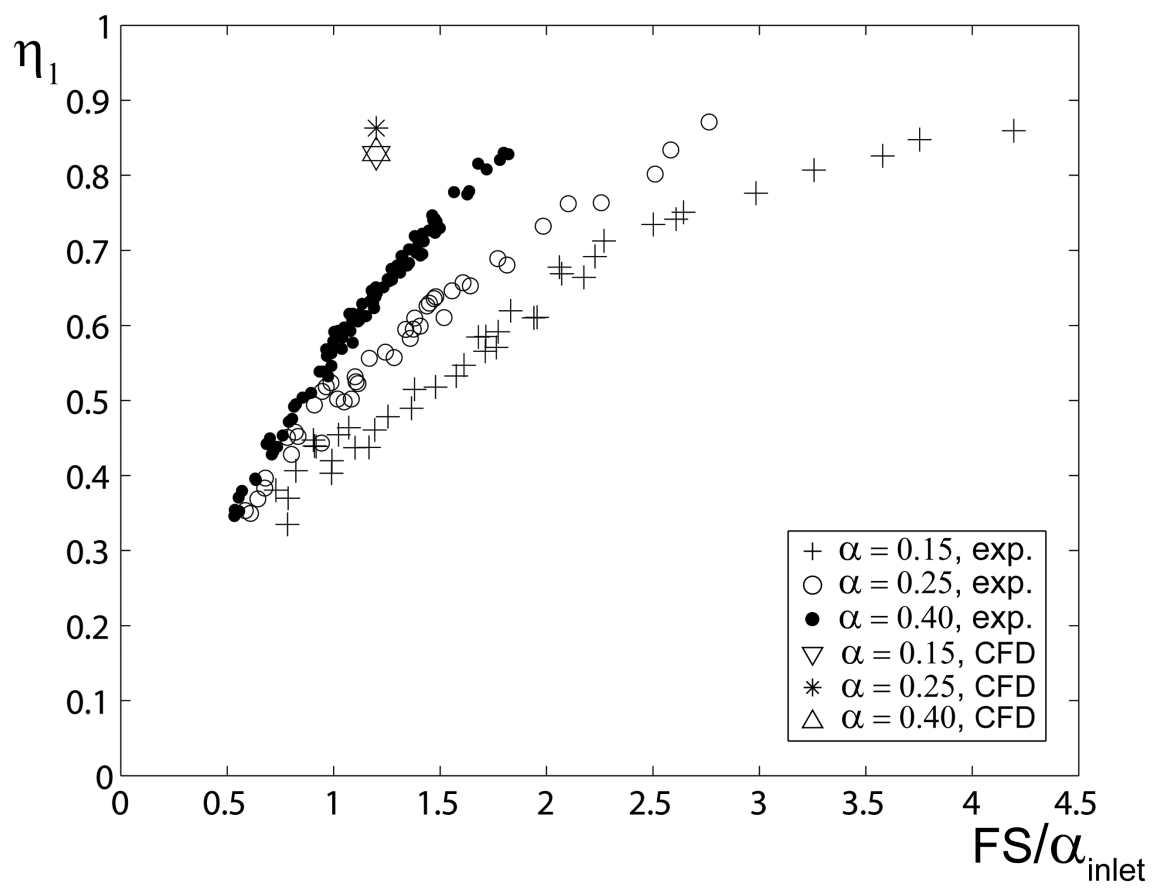

Figure 5.31: Comparison of separation efficiency $\eta_{1}$ as function of FS $/ \alpha_{\text {inlet }}$ from numerical simulations and from measurements for $\alpha_{\text {inlet }}=[0.15,0.25,0.40]$. Non-dimensional parameters used in numerical simulations are given in table 5.7. Experimental results by Laurens van Campen, TUD. 
fractions, since the space between the oil droplets is smaller leading more frequently to droplet-droplet collisions. In addition, in denser emulsions the time droplets are in contact during such a collision is likely to increase due to the lower velocity difference between the droplets. This longer contact time further increases the coalescence rate. The increase in droplet size will lead to a large reduction of the drag between the phases. Also, relatively large regions of continuous oil may be formed and in the center of the pipe phase inversion may be expected. For such a flow the modeling of the mixture viscosity as given in equation (2.97) is no longer appropriate. These flow features may explain the absence of the declining efficiencies for high $\alpha_{i n l e t}$ in the measured results since they facilitate the separation and prevent flooding.

Coalescence and phase inversion are flow features which lead to a drag between the phases that is different than predicted by the drag law. The numerical simulations of the microcentrifuge experiments also showed a higher oil volume fraction than the experimental data, see section 5.2.1. However, the emulsions in the micro-centrifuge have been stabilized to prevent coalescence, while in the experimental setup at the TUD coalescence and phase inversion can occur. Compared to the drag for a system with coalescence,

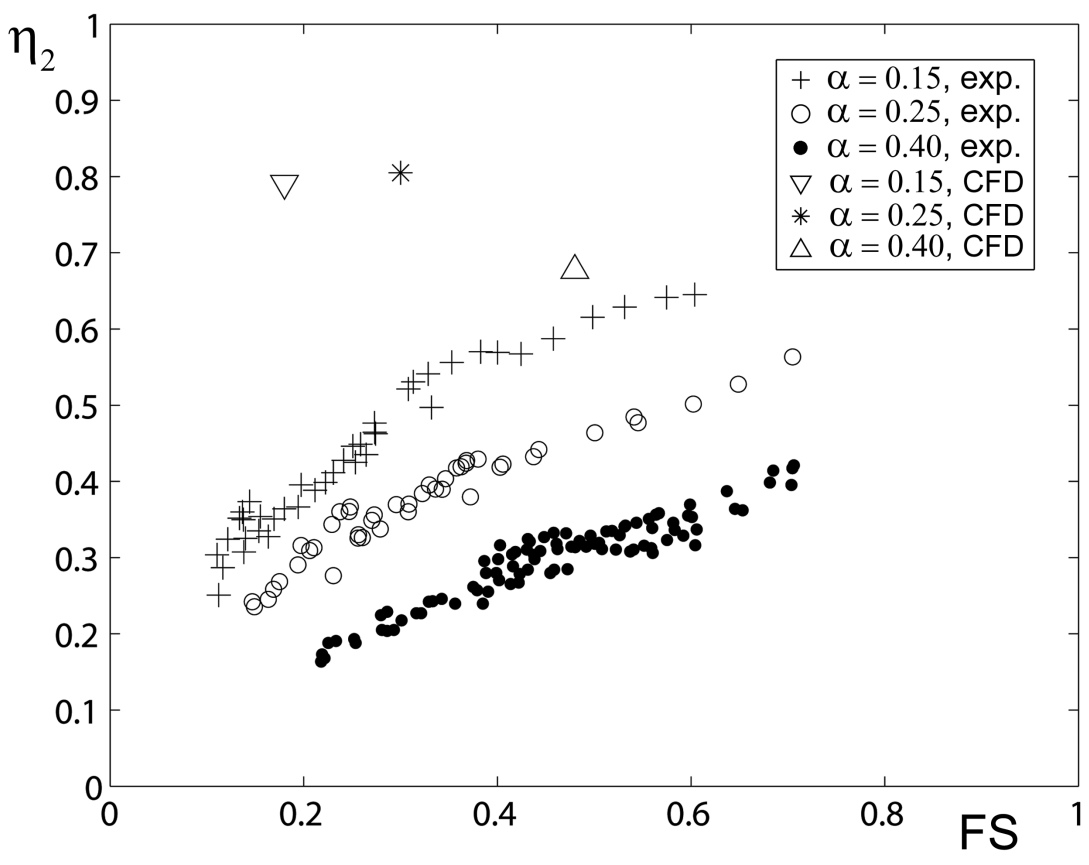

Figure 5.32: Comparison of separation efficiency $\eta_{2}$ as function of FS from numerical simulations and from measurements for $\alpha_{\text {inlet }}=[0.15,0.25,0.40]$. Non-dimensional parameters used in numerical simulations are given in table 5.7. Experimental results by Laurens van Campen, TUD. 
the Ishii-Zuber drag law with constant droplet diameter is expected to yield a drag that is too high. So based on the drag law formulation an underprediction of the separation efficiency is expected instead of an overprediction. Therefore, the formulation of the drag law is unlikely to be the cause of the overprediction of the separation efficiency.

The lack of a model for turbulent dispersion in the numerical simulations does provide an explanation for the overprediction of the separation efficiency. However, it is difficult to assess a priori the impact of turbulent dispersion on the separation efficiency. Experimental research into turbulent dispersion is challenging for the present system due to the high oil volume fraction, which prevents optical access to regions deeper into the separator. Moreover, it is nearly impossible to separate the effect of turbulent dispersion from other flow features. This complicates validation of turbulent dispersion models for flows with anisotropic turbulence.

The droplet size has a large influence on the separation efficiency. So a different (mean) droplet size at the inlet of the separator in the experiment compared to the one used in the numerical simulation will have a large influence on the separation performance. The droplet size at the inlet of the experimental setup is not easily controlled, however, prelim-

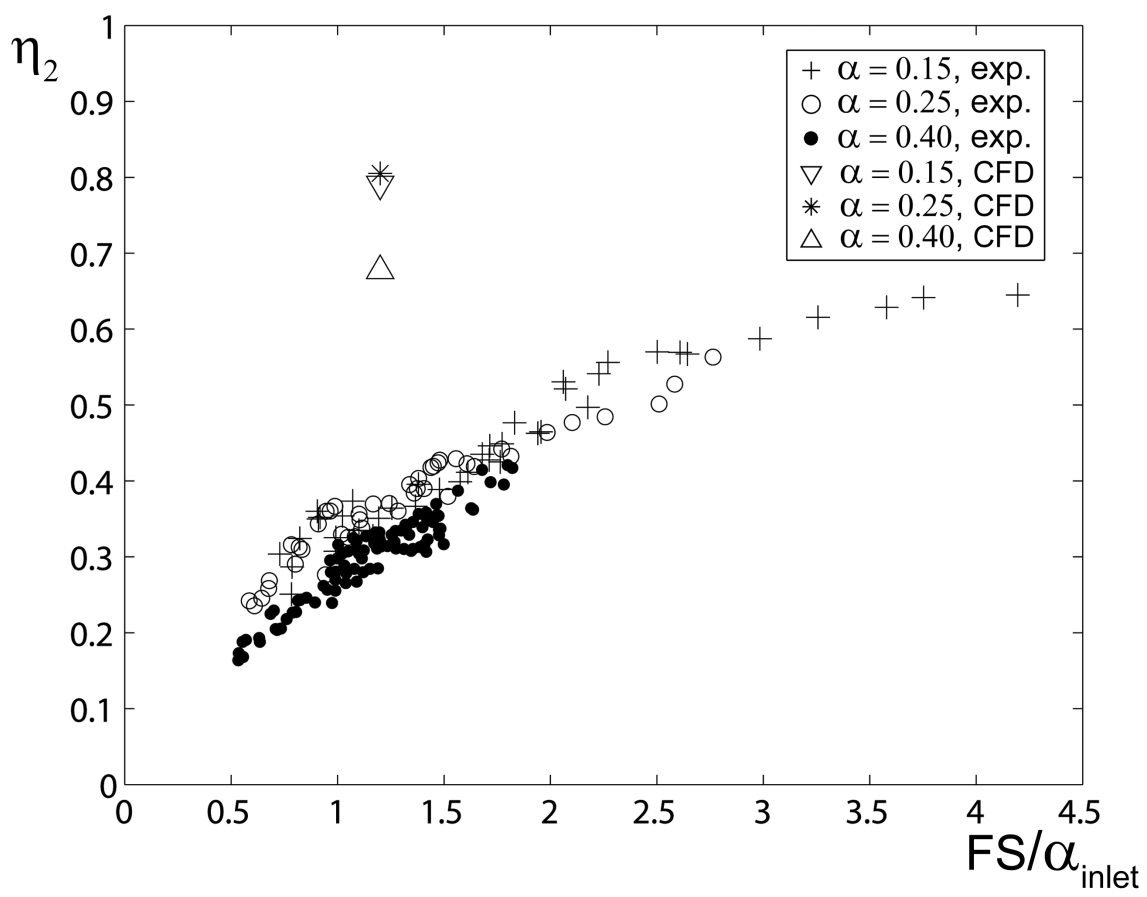

Figure 5.33: Comparison of separation efficiency $\eta_{2}$ as function of FS $/ \alpha_{\text {inlet }}$ from numerical simulations and from experimental measurements for $\alpha_{\text {inlet }}=[0.15,0.25,0.40]$. Non-dimensional parameters used in numerical simulations are given in table 5.7. Experimental results by Laurens van Campen, TUD. 
inary droplet size measurements pointed out that the mean droplet diameter is reasonably close to $100 \mu \mathrm{m}$, which corresponds with the size used in the numerical simulations.

The droplet diameter is assumed constant in the present numerical simulations. In largescale experiments a distribution of the droplet size will be present. Coalescence and break-up will change this distribution of the droplet size as the oil-water mixture flows through the separator. The preliminary droplet size measurements also point out that considerable droplet break-up occurs as the mixture flows through the ISE. High turbulent dissipation rates are observed near the surface of the ISE and in the detachment region on the afterbody. This turbulence deforms the droplets and may cause break-up of large droplets. In addition, the flow is accelerated in the vane section which stretches the oil droplets and this also may lead to break-up. To assess the effect of the droplet size on the separation performance a series of numerical simulations for different inlet droplet diameters has been performed. This is discussed in the next section.

\subsubsection{Variation of the inlet droplet size}

The effect on the separation efficiency of the droplet diameter is studied using $d=[50$, $75,100] \mu \mathrm{m}$. The effect of the smaller droplet diameter on the separation of oil from the oil-water mixture can be seen in figure 5.34. The maximum oil volume fraction can be

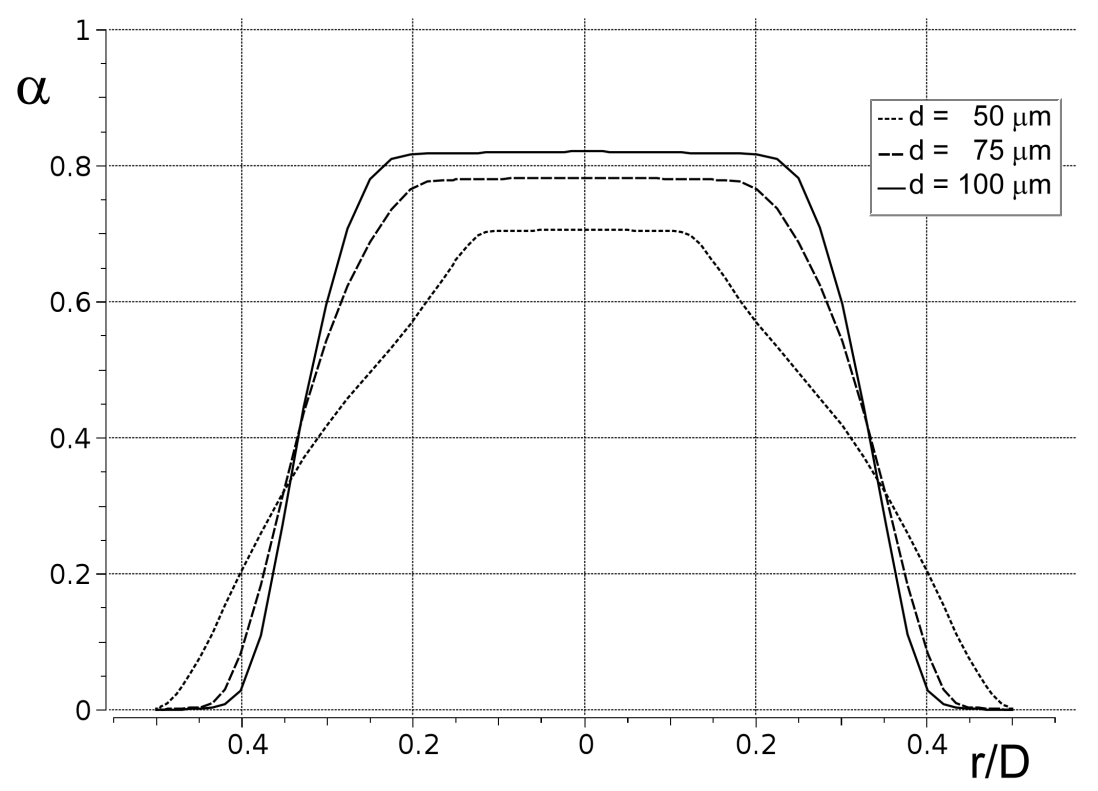

Figure 5.34: Radial distribution of the time-averaged oil volume fraction $\alpha$ on a line through the center of the separator in the plane $z=1.50 \mathrm{~m}$ for droplet diameter $d=[50,75,100] \mu \mathrm{m}$. Non-dimensional parameters are $\mathrm{FS}=0.30, \mathrm{Re}_{\mathrm{D}}=0.86 \times 10^{5}, \mathrm{Sw}=6.61, d / D=\left[\begin{array}{ll}0.50 & 0.75\end{array}\right.$ $1.00 \mathrm{~J} \times 10^{-3}$ and $\alpha_{\text {inlet }}=0.25$. 


\begin{tabular}{|c|c|c|c|c|c|c|c|}
\hline$d$ & $\alpha_{H P O}$ & $\alpha_{L P O}$ & hold up & $\eta_{1}$ & $\frac{\Delta Q_{o}}{Q_{o, \text { inlet }}}$ & $\eta_{2}$ & $\frac{1}{1-F S} \frac{\Delta Q_{o}}{Q_{o, \text { inlet }}}$ \\
$(\mu \mathrm{m})$ & $(\%)$ & $(\%)$ & $(\%)$ & $(\%)$ & $(\%)$ & $(\%)$ & $(\%)$ \\
\hline \hline 50 & 14 & 51 & 37 & 61 & 0.53 & 45 & 0.76 \\
75 & 7.6 & 65 & 41 & 79 & 0.38 & 70 & 0.54 \\
100 & 4.8 & 72 & 42 & 86 & 0.56 & 80 & 0.80 \\
\hline
\end{tabular}

Table 5.9: Results for different oil droplet diameter $d . \mathrm{FS}=0.30, \mathrm{Re}_{\mathrm{D}}=0.86 \times 10^{5}, \mathrm{Sw}=6.61$, $d / D=\left[\begin{array}{lll}0.50 & 0.75 & 1.00\end{array}\right] \times 10^{-3}$ and $\alpha_{\text {inlet }}=0.25$.

seen to increase with increasing droplet diameter. For $d=100 \mu \mathrm{m}$ the maximum value of $\alpha$ is higher than 0.80 , while for a droplet diameter of $50 \mu \mathrm{m}$ the maximum $\alpha$ does not become larger than about 0.70 . For the latter case oil-free water is not even obtained at the wall. Furthermore, for larger oil droplets the oil-rich core is narrower and the gradient of the oil volume fraction in the radial direction is larger.

The velocity distribution is nearly identical for the three droplet diameters. The axial
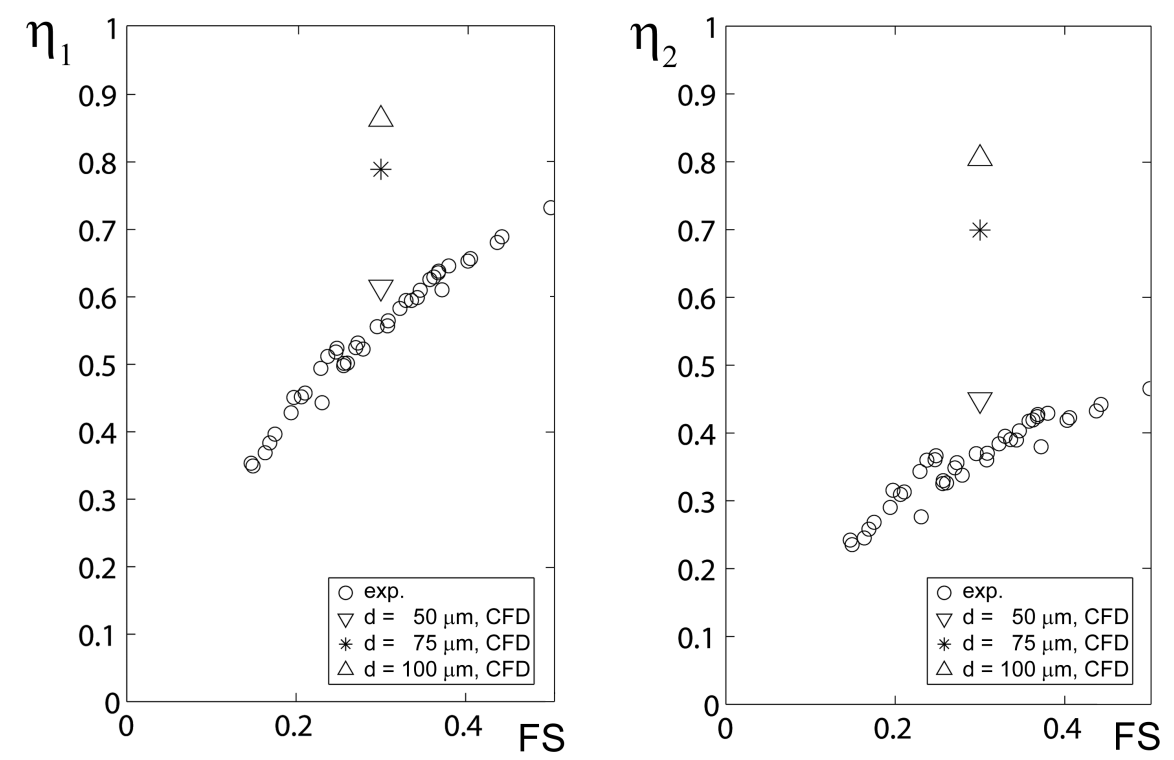

Figure 5.35: Comparison of separation efficiencies $\eta_{1}$ (left) and $\eta_{2}$ (right) as function of FS determined from numerical simulations and from measurements for $d=[50,75,100] \mu \mathrm{m}$. Non-dimensional parameters are $\mathrm{FS}=0.30, \mathrm{Re}_{\mathrm{D}}=0.86 \times 10^{5}, \mathrm{Sw}=6.61, d / D=\left[\begin{array}{ll}0.50 & 0.75\end{array}\right.$ $1.00] \times 10^{-3}$ and $\alpha_{\text {inlet }}=0.25$. 
velocity in the center of the pipe is slightly higher for smaller droplet diameter. However, the maximum difference in axial velocity for the cases of 50 and $100 \mu \mathrm{m}$ droplet diameter is only $5 \%$ of the bulk velocity $u_{z, b}$. So the distribution of the oil volume fraction has a limited influence on the distribution of the velocity. Also, the non-dimensional pressure drop $\mathrm{Eu}$ is nearly the same for this range in inlet droplet diameters with $\Delta \mathrm{Eu}<1 \%$.

The results of the calculations for different droplet diameter are shown in table 5.9. The separation efficiencies from table 5.9 are plotted together with the experimental results in figure 5.35. The comparison for $\eta_{1}$ is shown on the left and for $\eta_{2}$ on the right. Note that the horizontal axis is now clipped at $F S=0.50$. For both separation efficiencies the numerical results approach the experimental data when the droplet size is reduced. The preliminary droplet size measurements indicated that the smallest droplet size that exists in some reasonable quantity is about $50 \mu \mathrm{m}$. Smaller droplet are observed but only in very small quantities and are therefore not thought to have an noticeable influence on the separation efficiency. Therefore the mean droplet diameter in the experimental results is expected to be larger than $50 \mu \mathrm{m}$. Therefore the numerical simulations still overpredict the separation efficiency. However, the gap between numerical and experimental results is much smaller.

\subsubsection{Conclusions}

For two-phase flow the comparison between the results of numerical simulation and experiments show large differences in separation efficiency. The numerical simulations predict a much better performance of the separator than observed in the experiments at the TUD. A model to account for the turbulent dispersion is lacking in the numerical method used. However, the misrepresentation of the droplet size in the numerical simulations is expected to be the main cause for the deviation of the numerical results from the measurements. Even if a representative mean droplet diameter is used in the numerical simulations, it fails to account for the changes in the droplet size distribution caused by droplet coalescence and break-up. In order to more accurately predict the flow of oil-water mixtures incorporation of this evolution of the droplet size distribution in the numerical method is required. At least the variation of the mean droplet diameter should be taken into account. Therefore, in the next chapter two-phase flow simulations with a distribution of the droplet size and a varying mean droplet diameter will be explored. However, these models also contain many simplifications, which are based on many assumptions.

Besides the value of the separation efficiency, also the predicted trend for increasing inlet oil volume fraction differs from experimental findings. The cause for this may be found in the formulation of the drag law. The assumption of spherical-like oil droplets of certain diameter in a continuous water phase is unlikely to hold in the dense emulsion present in the center of the pipe. Again, the lack of numerical methods for the evolution of the droplet size distribution reduce the accuracy of the predictions. Adaptations could be made by incorporating, for instance, a mixture viscosity that accounts for phase inversion. However, criteria for phase inversion must then be developed. Small-scale emulsion tests at the TUD and the WUR have shown that these criteria are far from trivial since phase inversion depends on factors, such as the droplet size distribution, initial conditions, tur- 
bulence dissipation rates and many other factors.

Many of these issues can in principle be resolved by incorporating more physics and therefore more details, in the models of the numerical method. However, the computational time required for a typical numerical simulation of two-phase flow discussed in this chapter is about 20 to 30 days using 20 to 24 CPU's on a present-day computer cluster. Thus for the present application the computational cost are already quite high and numerical methods that incorporate more physics, such as volume of fluid methods (VOF) or large eddy simulations (LES) for two-phase flow are not feasible in the near future. Therefore, the current two-fluid RANS method is thought to be the best method that is currently feasible. Nonetheless, the end-user should be aware of the large deviations between numerical results and experimental results. 
CHAPTER 6

\section{Coalescence and break-up}

\subsection{Introduction}

The accurate prediction of the separation efficiency depends on an accurate prediction of the relative velocity between the phases. One of the key parameters in the modeling of the interaction of the phases is the droplet diameter. Its importance has been shown in section 5.4.4. So far, a constant droplet diameter has been used in the numerical simulation of two-phase flow. In reality, a distribution of the droplet size is present at the inlet and the size distribution alters in the separator due to the non-uniform flow and coalescence and break-up of the droplets. This evolution of the distribution of the droplet size can be predicted by the use of population balance equations (PBE).

The development of these methods started in the 1960s in the field of chemical engineering. Various types of PBE have been developed since then [54]. The first population balance method was presented by Hulburt and Katz [51]. They formulated equations describing the nucleation, growth and agglomeration of solid particles using concepts from classical statistical mechanics.

Here, the method of classes will be used, which is a method based on a macroscopic rather than a microscopic approach. This development was initiated by Coulaloglou and Tavlarides [23]. Subsequent modeling efforts produced many PBE formulations, especially for gas-liquid flows, as summarized by Liao [68] and Jakobsen [54]. In the method of classes the droplet size distribution is divided into droplet size groups. Transport equations for these groups are provided and coalescence and break-up models account for the mass transfer between the size groups. An advantage of the method of classes is that the droplet size distribution is directly known [6] and that the method is computationally robust [4]. 
For flows with large differences in droplet size, many droplet size groups may be required in order to accurately describe the droplet size distribution. The large number of droplet size groups may lead to excessive computational costs. This can be resolved by employing the method of moments (MOM), which describes the evolution of the droplet size distribution using an limited number of moments of that distribution [76]. The equations for the moments do not provide information on the shape of the distribution, which presents a closure problem. The integrals in the moment equations can be approximated by Gaussian quadrature, this yields the quadrature method of moments (QMOM). An improvement to this method is the direct quadrature method of moments (DQMOM) by Machisio and Fox [73].

DQMOM gives results comparable to the results of the methods of classes, while DQMOM is much more computationally efficient $[96,100]$. Thus, DQMOM is expected to become the preferred method for PBE in the future. However, an implementation of DQMOM is currently not available in Ansys CFX and the implementation of DQMOM with a user-defined subroutine in Ansys CFX is beyond to scope of the present project. Therefore, the method of classes will be employed here.

\subsection{Population balances}

\subsubsection{Number density equations}

The starting point of the derivation of the PBE is the transport equation of the number density $n_{p}\left(\left[n_{p}\right]=1 / \mathrm{m}^{3}\right)$ of droplets of mass $m_{p}$

$$
\frac{\partial n_{p}}{\partial t}+\nabla \cdot\left(\mathbf{u}_{p} n_{p}\right)=B_{B, p}-D_{B, p}+B_{C, p}-D_{C, p}
$$

Here $\mathbf{u}_{p}$ is the velocity of droplets of size $p$. The first source terms on the right-hand side is the birth by break-up term $B_{B, p}\left(\left[B_{B, p}\right]=1 / \mathrm{m}^{3} \mathrm{~s}\right)$, which accounts for the increase in $n_{p}$ caused by the break-up of larger droplets

$$
B_{B, p}=\sum_{q=p+1}^{\max } g\left(m_{q}: m_{p}\right) n_{q}
$$

The specific break-up rate $g\left(m_{q}: m_{p}\right)([g]=1 / \mathrm{s})$ gives the rate at which droplets of mass $m_{q}$ break-up into droplets of mass $m_{p}$ and $m_{q}-m_{p}$. Here $m_{\max }$ is the mass of the droplets in the class with the largest droplets. Break-up of droplets of mass $m_{p}$ are accounted for in the death by break-up term $D_{B, p}$

$$
D_{B, p}=n_{p} \sum_{q=m i n}^{p-1} g\left(m_{p}: m_{q}\right)
$$

Here $m_{\min }$ is the mass of the droplets in the class with the smallest droplets. The birth by coalescence term $B_{C, p}$ gives the increase of the number density $n_{p}$ due to coalescence 
of smaller droplets into a droplet of mass $m_{p}$

$$
B_{C, p}=\frac{1}{2} \sum_{q=m i n}^{p-1} Q\left(m_{p}-m_{q}: m_{q}\right) n_{p-q} n_{q}
$$

Here $Q\left(m_{p}-m_{q}: m_{q}\right)\left([Q]=\mathrm{m}^{3} / \mathrm{s}\right)$ is the specific coalescence rate at which droplets of mass $m_{p}-m_{q}$ and $m_{q}$ coalesce into droplets of mass $m_{p}$. Since $Q\left(m_{p}-m_{q}: m_{q}\right)$ and $Q\left(m_{q}: m_{p}-m_{q}\right)$ involve the same droplet sizes, the factor $1 / 2$ is introduced. Evidently, a droplet of mass $m_{p}$ can collide with other droplets and coalesce into large droplets. This rate is given by the death by coalescence term $D_{C, p}$

$$
D_{C, p}=n_{p} \sum_{q=\min }^{\max } Q\left(m_{p}: m_{q}\right) n_{q}
$$

In principle, every droplet size has its own number density $n_{p}$. In practice many droplet sizes exists in the flow and computing a transport equation for all number densities will be computationally impossible. Therefore size groups are formed, which consist out of droplets within a range of droplet sizes. The number density $N_{p}$ of size group $p$ with characteristic mass $M_{p}$ is given by

$$
N_{p}=\sum_{p^{\prime}=r}^{s} n_{p^{\prime}}
$$

Here $r$ and $s$ are the smallest and largest droplet sizes of the range of droplet sizes which constitute size group $p$. If the summation operation of equation (6.6) is applied to equation (6.1) and the result is multiplied by $M_{p}$, while realizing that $M_{p} N_{p}=\rho_{o} \alpha f_{p}$, the following transport equation is obtained

$$
\frac{\partial\left(\alpha f_{p}\right)}{\partial t}+\nabla \cdot\left(\mathbf{u}_{d} \alpha f_{p}\right)=\frac{1}{\rho_{o}}\left(B_{B, p}^{\prime}-D_{B, p}^{\prime}+B_{C, p}^{\prime}-D_{C, p}^{\prime}\right)
$$

The size group fraction $f_{p}$ of size group $p$ is given by

$$
f_{p}=\frac{\alpha_{p}}{\alpha}
$$

Here $\alpha_{p}$ is the volume fraction of droplet size group $p$. The source terms can be rewritten straightforwardly into source terms for the size groups by summing over the size groups instead of over the droplet sizes. The result for the break-up terms $B_{B, p}^{\prime}$ and $D_{B, p}^{\prime}\left(\left[B_{B, p}^{\prime}\right]\right.$ $=\mathrm{kg} / \mathrm{m}^{3} \mathrm{~s}$ ) in the equation for size group $p$ are

$$
\begin{aligned}
& B_{B, p}^{\prime}=\rho_{o} \alpha \sum_{q=p+1}^{\max } g\left(M_{q}: M_{p}\right) f_{q} \\
& D_{B, p}^{\prime}=\rho_{o} \alpha f_{p} \sum_{q=\min }^{p-1} g\left(M_{p}: M_{q}\right)
\end{aligned}
$$


If the mass of a droplet formed by coalescence does not coincide with the averaged mass of one of the size groups, then the mass flux must be distributed between adjacent size groups. The fraction of the mass flowing into size group $p$ for coalescence events between droplets of mass $M_{q}$ and $M_{r}$ is given by

$$
X_{p q r}= \begin{cases}\frac{\left(M_{q}+M_{r}\right)-M_{p-1}}{M_{p}-M_{p-1}} & \text { if } M_{p-1} \leq\left(M_{q}+M_{r}\right)<M_{p} \\ \frac{M_{p+1}-\left(M_{q}+M_{r}\right)}{M_{p+1}-M_{p}} & \text { if } M_{p}<\left(M_{q}+M_{r}\right)<M_{p+1} \\ 0 & \text { otherwise }\end{cases}
$$

For the cases for which $M_{p-1}<M_{q}+M_{r}<M_{p}$ the distribution is graphically explained in figure 6.1 . The birth by coalescence term is now given by

$$
B_{C, p}^{\prime}=\frac{1}{2}\left(\rho_{o} \alpha\right)^{2} \sum_{q=\min }^{p-1} \sum_{r=\min }^{p-1} Q\left(M_{q}: M_{r}\right) X_{p q r} f_{q} f_{r} \frac{M_{q}+M_{r}}{M_{q} M_{r}}
$$

And finally the death by coalescence term $D_{C, p}^{\prime}$ is given by

$$
D_{C, p}^{\prime}=\left(\rho_{o} \alpha\right)^{2} \sum_{q=\min }^{\max } Q\left(M_{p}: M_{q}\right) f_{p} f_{q} \frac{1}{M_{q}}
$$

The next and crucial step is to determine the specific coalescence rate $Q$ and the specific break-up rate $g$.

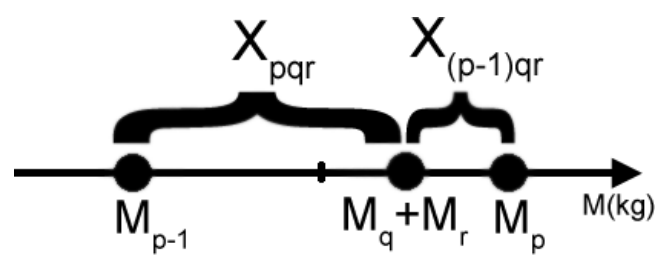

Figure 6.1: Graphical depiction of distribution of inflowing mass between size group $M_{p-1}$ and $M_{p}$ after coalescence of droplets $M_{q}$ and $M_{r}$ for $M_{p-1}<M_{q}+M_{r}<M_{p}$. 


\subsubsection{Turbulence-induced coalescence}

In a turbulent flow, the motion of the oil droplets is affected by turbulent eddies. These eddies can cause collisions between droplets, which in turn may lead to coalescence. Three stages are distinguished in a coalescence event [112]. These are:

- the approach of the droplets

- the drainage of the continuous film between the droplets that are very close to each other

- the film rupture

The motion of the droplets due to turbulence is often the only coalescence mechanism accounted for in turbulent flows [68]. In Ansys CFX, the turbulence-induced coalescence model of Prince and Blanche [84] is used. This model has been originally developed for bubble columns. The model by Prince and Blanche therefore assumes fully mobile interfaces, since this behavior is seen during film drainage in these bubble systems. A fully mobile interface between the droplet and the continuous fluid allows for circulation of the fluid inside the droplet during the draining of the film before coalescence. This circulation greatly facilitates the drainage of the film and therefore shortens the coalescence time. The distribution of the velocity in the film for three types of interface mobility are sketched in figure 6.2. In the oil-water systems considered here, the viscosity of the oil is much higher than the viscosity of the water. Furthermore, it is expected that surface-active contaminants will be present. These contaminants decrease the interface mobility even for low concentrations of the contaminants. Therefore, the interface is at best partially mobile and an immobile interface can be assumed for the derivation of a conservative estimate of the coalescence time scale. Therefore, a different model has been used to
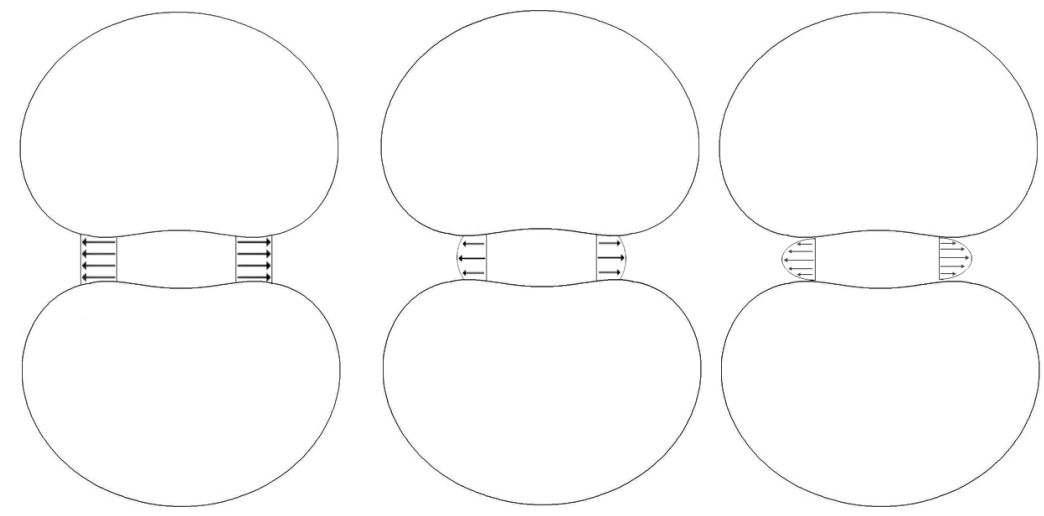

Figure 6.2: Distribution of the velocity in film during drainage of the film between two colliding large droplets of equal size, for three types of interface mobility: fully mobile (left), partially mobile (middle) and immobile (right). 
model the turbulence-induced coalescence. A suitable model is presented by Coulaloglou and Tavlarides [23]. This widely-used model is developed for coalescence between liquid droplets with immobile surfaces in stirred vessels. Fortunately, it is applicable for the range of turbulent dissipation rates observed in the separator. The specific coalescence rate is modeled as the product of the specific collision frequency $\theta\left([\theta]=\mathrm{m}^{3} / \mathrm{s}\right)$ and the coalescence efficiency $\lambda$. The collision efficiency $\lambda$ gives the probability of coalescence in the event of a collision. The specific coalescence rate is given by

$$
Q\left(M_{p}: M_{q}\right)=\theta_{p q} \lambda_{p q}
$$

The mechanism of droplet collisions is assumed to be similar to that of collisions between molecules, as described in the kinetic gas theory [17].

$$
\theta_{p q}=\frac{\pi}{4}\left(d_{p}+d_{q}\right)^{2}\left(u_{p}^{\prime 2}+u_{q}^{\prime 2}\right)^{1 / 2}
$$

Here $d_{p}$ and $d_{q}$ are the droplet diameters pertaining to mass $M_{p}$ and $M_{q}$, respectively. The magnitude of the average fluctuating velocity of droplet $d_{p}$ is $u_{p}^{\prime}$. Note that only binary collisions are considered here. Also note that for dense emulsions the assumed similarity of the collision mechanism for droplets to that of molecules is less appropriate. The fluctuating velocities of the droplets are caused by eddies of a size similar to that of the droplets. A large eddy, compared to the size of the size of the droplet, will simply transport the droplet, while a small eddy will impart little movement on to the droplet. Some assumptions are made to arrive at an expression for the fluctuating velocity. It is assumed that the fluctuating velocity of the droplet is equal to the velocity of the interacting eddy. Since the disperse phase is lighter than the continuous phase, this assumption will most probably be valid. Also, the turbulence is assumed to be isotropic. The flow encountered here does not feature isotropic turbulence, however, at the smaller scales the turbulence will be more isotropic. Thus the assumption of isotropic turbulence is probably not that far from reality. Also the size of the droplets, and therefore of the eddies, are stated to be within the inertial subrange of turbulence. However, for the turbulent dissipation levels seen in the separator, the droplet sizes are smaller than the typical length scale of $60 \eta$, which divides the dissipation range and the inertial subrange [82]. Here $\eta$ is the Kolmogorov length scale, defined as

$$
\eta=\left(\frac{\nu^{3}}{\epsilon}\right)^{\frac{1}{4}}
$$

In the flow in the separator it is expected that $10<\eta<20 \mu \mathrm{m}$. However, since this length scale is an order of magnitude estimate it is assumed that the error made by using the turbulence characteristics of the inertial subrange to estimate the fluctuating velocity is relatively small. Based on turbulence theory one then finds [58]

$$
u_{p}^{\prime 2} \sim\left(\epsilon d_{p}\right)^{2 / 3}
$$

Coulaloglou and Tavlarides [23] found difficulty in correlating the outcome of the theoretical model to experimental results for cases with relatively high volume fractions of 
the dispersed phase. This comparison improved after they accounted for the damping of turbulence by the presence of many small droplets. The damping of the fluctuating velocity is approximated by

$$
u_{p}^{\prime 2} \sim \frac{\left(\epsilon d_{p}\right)^{2 / 3}}{(1+\alpha)^{2}}
$$

Finally, substitution of equation (6.18) into equation (6.15) and multiplying with an empirical constant $C_{3}$ leads to the specific collision frequency

$$
\theta_{p q}=C_{3} \frac{\epsilon^{1 / 3}}{(1+\alpha)}\left(d_{p}+d_{q}\right)^{2}\left(d_{p}^{2 / 3}+d_{q}^{2 / 3}\right)^{1 / 2}
$$

The coalescence efficiency $\lambda_{p q}$ between droplets of size $d_{p}$ and $d_{q}$ is usually modeled by an exponential function depending on the time scale of coalescence $t_{p q}$ and the timescale of the collision or contact time $\tau_{p q}$ [23].

$$
\lambda_{p q}=e^{-t_{p q} / \tau_{p q}}
$$

Evidently, the likelihood of coalescence decreases as the time necessary for coalescence increases. According to Coulaloglou and Tavlarides [23] the timescale of coalescence of two deformable droplets $d_{p}$ and $d_{q}$ scales as

$$
t_{p q} \sim \frac{\mu_{w} F}{\sigma^{2}}\left(\frac{1}{h_{f}^{2}}-\frac{1}{h_{0}^{2}}\right)\left(\frac{d_{p} d_{q}}{d_{p}+d_{q}}\right)^{2}
$$

Here $F$ is the compressive force, $h_{f}$ and $h_{0}$ are film thicknesses at which the film ruptures and at which the drainage starts, respectively. Both $h_{0}$ and $h_{f}$ are assumed to be constants. The term $\left(d_{p} d_{q}\right) /\left(d_{p}+d_{q}\right)$ is the equivalent radius as defined by Chesters [19]:

$$
r_{p q}=2\left(\frac{1}{r_{p}}+\frac{1}{r_{q}}\right)^{-1}=2\left[\frac{r_{p} r_{q}}{r_{p}+r_{q}}\right]=\frac{d_{p} d_{q}}{d_{p}+d_{q}}
$$

Note that the coalescence time increases with the compressive force $F$, which drives the film drainage. This is due to the stronger deformation of the droplet for higher $F$. A larger deformation of the droplets creates a larger surface area of the film between the droplets, which leads to a higher flow resistance against film drainage. The compressive force $F$ is caused by inertial collision between the droplets caused by the action of turbulent eddies. From dimensional analysis one finds that the force $F$ scales with the density of the continuous fluid times the square of a characteristic velocity scale $\mathcal{U}$ times the square of a characteristic length scale $\mathcal{L}$ [110], that is

$$
F \sim \rho_{w}(\mathcal{U L})^{2}
$$

Coulaloglou [23] defines the velocity scale as

$$
\mathcal{U}^{2}=\frac{\epsilon^{2 / 3}}{(1+\alpha)^{2}}\left(d_{p}+d_{q}\right)^{2 / 3}
$$


and the length scale as

$$
\mathcal{L}=\frac{d_{p} d_{q}}{d_{p}+d_{q}}
$$

This lead to

$$
F \sim \rho_{w} \epsilon^{2 / 3} \frac{\left(d_{p}+d_{q}\right)^{2 / 3}}{(1+\alpha)^{2}}\left(\frac{d_{p} d_{q}}{d_{p}+d_{q}}\right)^{2}
$$

Coulaloglou and Tavlarides [23] define the collision time-scale as

$$
\tau_{p q} \sim \frac{\left(d_{p}+d_{q}\right)^{2 / 3}}{\epsilon^{1 / 3}}(1+\alpha)
$$

Substitution into equation (6.20) and the inclusion of an empirical constant $C_{4}$ leads to

$$
\lambda_{p q}=\exp \left(-C_{4} \frac{\mu_{w} \rho_{w} \epsilon}{\sigma^{2}(1+\alpha)^{3}}\left(\frac{d_{p} d_{q}}{d_{p}+d_{q}}\right)^{4}\right)
$$

Quite a few sets of the values of the constants $C_{3}$ and $C_{4}$ exist and most sets are rather similar. Here the original constants of Coulaloglou and Tavlarides are used: $C_{3}=$ $2.17 \times 10^{-4}, C_{4}=2.28 \times 10^{13} \mathrm{~m}^{-2}$.

The effect of using the model by Coulaloglou and Tavlarides instead of the model by Prince and Blanch can be assessed by considering the difference in coalescence efficiency. The time-scale of coalescence $t_{p q}^{P B}$ in the model of Prince and Blanche is given by

$$
t_{p q}^{P B}=\sqrt{\frac{r_{p q}^{3} \rho_{w}}{16 \sigma}} \ln \left(\frac{h_{0}}{h_{f}}\right)
$$

The contact time $\tau_{p q}^{P B}$ is derived from dimensional analysis of turbulent flow and is given by

$$
\tau_{p q}^{P B}=\frac{r_{p q}^{2 / 3}}{\epsilon^{1 / 3}}
$$

Substitution of the time scales into equation (6.20) leads to

$$
\lambda_{p q}^{P B}=\exp \left(-\frac{1}{4} \ln \left(\frac{h_{f}}{h_{0}}\right) \sqrt{\frac{\rho_{w} \epsilon^{2 / 3}}{\sigma}}\left(\frac{d_{p} d_{q}}{d_{p}+d_{q}}\right)^{5 / 6}\right)
$$

The coalescence efficiencies can now be examined for a range of droplet sizes and levels of turbulent dissipation rate $\epsilon$. The material properties are fixed and are chosen as $\rho_{w}=$ $1067.8 \mathrm{~kg} / \mathrm{m}^{3}, \mu_{w}=1.183 \times 10^{-3}$ Pa.s and $\sigma=0.021 \mathrm{~N} / \mathrm{m}$. Moreover, $h_{0}=100 \mu \mathrm{m}$ and $h_{f}=0.01 \mu \mathrm{m}$ [84]. Also, for fair comparison, the attenuation of the turbulence given in equation (6.18) is not taken into account. Therefore, the comparison can be thought of as for dilute systems. An iso-contour plot of the ratio $\lambda_{p q} / \lambda_{p q}^{P B}$ is shown in figure 6.3 for droplet sizes in the range $10-200 \mu \mathrm{m}$. The turbulent dissipation rate is set equal to $\epsilon=25 \mathrm{~m}^{2} / \mathrm{s}^{3}$, which is a representative value for the turbulent dispersion rate in the 
intermediate region $r>25 \mathrm{~mm}$, except very close to the pipe wall. In figure 6.4 the ratio $\lambda_{p q} / \lambda_{p q}^{P B}$ is shown for equally sized droplets $d_{p}=d_{q}$, while the turbulent dissipation rate is varied from unity to $250 \mathrm{~m}^{2} / \mathrm{s}^{3}$.

Figures 6.3 and 6.4 show that the newly implemented coalescence model predicts a coalescence efficiency that is much lower than the one obtained by the model of Prince and Blanche applied for flow conditions and material properties encountered in the current application. For droplets larger than $75 \mu \mathrm{m}$, the coalescence efficiency $\lambda_{p q}$ is at least an order of magnitude lower than $\lambda_{p q}^{P B}$. The difference can be attributed to the larger deformation of the droplets, which creates a larger film area in the contact region between the droplets. This affects the film drainage more drastically for immobile surfaces. Larger droplets, especially in combination with lower interfacial tension, deform more easily. Also, a higher turbulent dissipation rate $\epsilon$ and the higher velocity of the larger eddies, which transports the larger droplets, lead to more forceful collisions and therefore larger deformations.

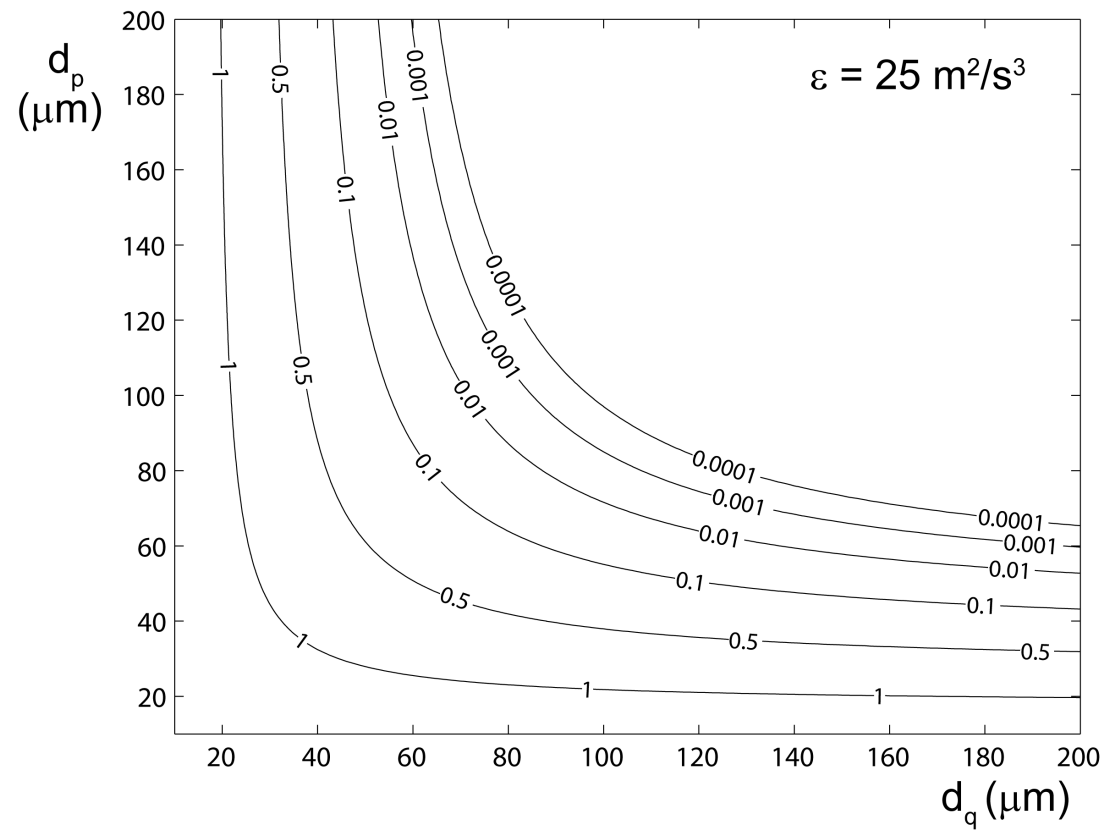

Figure 6.3: iso-contours of ratio of coalescence efficiencies $\lambda_{p q} / \lambda_{p q}^{P B}$ with $\lambda_{p q}$ and $\lambda_{p q}^{P B}$ given by equations (6.28) and (6.31), respectively, as function of the droplet size $d_{p}$ and $d_{q}$ of colliding droplets for a turbulent dissipation rate of $\epsilon=25 \mathrm{~m}^{2} / \mathrm{s}^{3}$. Input parameters are: $h_{0}=100 \mu \mathrm{m}$, $h_{f}=0.01 \mu \mathrm{m}, \mu_{w}=1.183 \times 10^{-3}$ Pa.s, $\rho_{w}=1067.8 \mathrm{~kg} / \mathrm{m}^{3}, \sigma=0.021 \mathrm{~N} / \mathrm{m}$. 


\subsubsection{Droplet break-up}

Large droplets may break-up into smaller ones, which are more difficult to separate. At the TUD droplet size measurements have been carried out. These measurements show that droplet break-up occurs in the separator. Therefore, incorporating droplet break-up accurately into the numerical simulations is expected to considerably improve the accuracy of the predictions.

\section{Theory}

Due to the deformation of the droplet, its surface area increases. Consequently, the interfacial energy of the droplet is increased. If the deformation is large enough the surface area can be reduced by droplet break-up. The deformation is caused by the external flow field. This can be by strain rates in the mean velocity field, for instance caused by elongation of the flow and by shear layers. Also the deformation can be caused by turbulent fluctuations.

In the vane section of the ISE the flow is strongly accelerated and break-up may occur due to elongation of the droplets. However, since the flow is highly turbulent, break-up

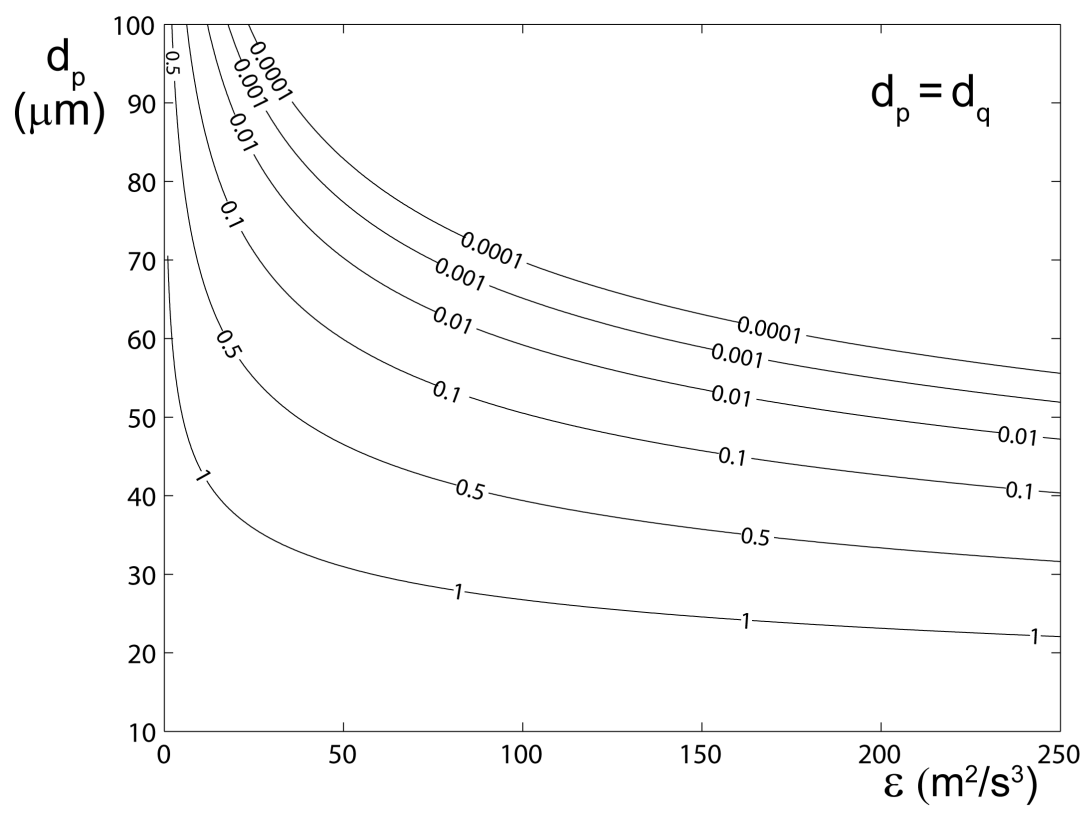

Figure 6.4: Iso-contour of ratio of coalescence efficiencies $\lambda_{p q} / \lambda_{p q}^{P B}$ with $\lambda_{p q}$ and $\lambda_{p q}^{P B}$ given by equations (6.28) and (6.31), respectively, for equal-sized droplets as function of the droplet size $d_{p}$ and the turbulent dissipation $\epsilon$. Input parameters are: $h_{0}=100 \mu \mathrm{m}, h_{f}=0.01 \mu \mathrm{m}, \mu_{w}=$ $1.183 \times 10^{-3}$ Pa.s, $\rho_{w}=1067.8 \mathrm{~kg} / \mathrm{m}^{3}, \sigma=0.021 \mathrm{~N} / \mathrm{m}$. 
is expected to be caused mainly by turbulent fluctuations.

The interaction of a turbulent eddy with a droplet can lead to two types of deforming forces; tangential stress and pressure forces. The droplets are subjected to tangential stresses $\tau_{\mu}$, stemming from viscosity. An estimate for $\tau_{\mu}$ is given by

$$
\tau_{\mu} \propto \frac{\mu_{w} \Delta u}{d}
$$

Here $\Delta u$ is the velocity difference over the droplet of diameter $d$ induced by turbulent eddies. Moreover, normal stresses $\tau_{\text {pres }}$ are acting on the droplets due to the pressure fluctuations. This stress scales as

$$
\tau_{\text {pres }} \propto \rho_{w}(\Delta u)^{2}
$$

The relative importance of the external stresses can be judged from the droplet Reynolds number $\operatorname{Re}_{\mathrm{d}}$, which is the ratio between equations (6.32) and (6.33):

$$
\operatorname{Re}_{\mathrm{d}}=\frac{\rho_{w} \Delta u d}{\mu_{w}}
$$

In the separator $\mathrm{Re}_{\mathrm{d}}$ is expected to be in the order of 1 to 10 , which is not that large. The external viscous stress is counteracted by the viscous stresses arising from the movement of the oil in the droplet $\tau_{i n}$ :

$$
\tau_{i n} \propto \frac{\mu_{d} u_{i n}}{d}
$$

Here $u_{i n}$ is the velocity of the oil in the droplet. The deformation induced by the fluctuating pressure is opposed by the Laplace pressure due to the interfacial tension. The later stress $\tau_{i f}$ is given by

$$
\tau_{i f} \propto \frac{4 \sigma}{d}
$$

In the present case the viscosity ratio is $\mu_{o} / \mu_{w}$ is higher than 15 . This high viscosity ratio makes it more difficult to break-up the droplet by viscous stress, as has been found by Taylor [109]. Also Davies [26] states that simple shear and stretching cannot form emulsions for viscosity ratios $\mu_{o} / \mu_{w}>3.5$. In these cases the time required for elongation of the droplet is longer than the lifetime of the eddy. Therefore, mainly the turbulent pressure fluctuations are expected to be responsible for the break-up. Equations (6.33) and (6.36) are thus used to estimate the stresses, which are acting on the droplet. Equations (6.33) and (6.36) can be combined to give the Weber number We.

$$
\mathrm{We}=\frac{\rho_{w}(\Delta u)^{2} d}{\sigma}
$$

One can define a critical Weber number based on the maximum size of the droplets $d_{\max }$ that can occur in the flow

$$
\mathrm{We}_{\mathrm{cr}}=\frac{\rho_{w}(\Delta u)^{2} d_{\max }}{\sigma}
$$


Larger drops, with correspondingly larger Weber numbers, will break-up into smaller droplets. It can be shown that in the inertial subrange the velocity difference is given by [7]

$$
(\Delta u)^{2}=2.0\left(\epsilon l_{e}\right)^{2 / 3}
$$

Thus the energy of an eddy increases with its size $l_{e}$. Also, it is assumed that eddies, larger than the droplet, simply transport the droplet. Therefore, the eddies of the same size as the droplet are most capable to break-up the droplet. Thus $l_{e}$ is equal to $\mathrm{d}_{\max }$. This leads to

$$
d_{\max }=\left(\frac{\mathrm{We}_{\mathrm{cr}}}{2}\right)^{3 / 5}\left(\frac{\sigma}{\rho_{w}}\right)^{3 / 5} \epsilon^{-2 / 5}
$$

Based on experiments with two co-axial cylinders, of which the inner cylinder is rotating, Hinze finds a critical Weber number $\mathrm{We}_{\mathrm{cr}}=1.2$ [47]. In that experiment the turbulence is assumed to be isotropic. However, in the separator the turbulence is anisotropic, but at the length scale of the droplets the flow will be approximately isotropic.

Hinze also assumes a low viscosity of the dispersed phase. However, the high viscosity ratio in the present case must be accounted for. Davies [26] adds to the interfacial tension of equation (6.36), an additional 'resistance' term in which the droplet viscosity is divided by the timescale of the eddy, that is the available break-up time:

$$
\tau_{D a v}=\frac{4 \sigma}{d}+\frac{\mu_{d} \Delta u}{d}
$$

Davies used the relation $\Delta u=\left(\epsilon d_{\max }\right)^{1 / 3}$ in his derivation. Now $\Delta u$ is the characteristic velocity scale of an eddy. The deformation-resisting stress as defined by Davies can be used for an alternative definition of the Weber number. Rewriting the expression in equation (6.40) yields

$$
d_{\text {max }}=C_{\text {Dav }}\left(\sigma+\frac{\mu_{d}\left(\epsilon d_{\text {max }}\right)^{1 / 3}}{4}\right)^{3 / 5} \rho_{w}^{-3 / 5} \epsilon^{-2 / 5}
$$

Davies sets the constant $C_{D a v}$ to unity. In the limit of inviscid dispersed flow, this expression therefore deviates from Hinze's results. Vankova et al. [114] and van der Zande [118] found that equation (6.42) predicts the maximum droplet size quite well compared to their experimental data, although they adjusted the constant $C_{D a v}$ to obtain a better fit.

These correlations only give a quick estimate of the maximum droplet size in the flow. However, for PBE a set of equations is required that describes the evolution of the droplet size distribution. Such a model is provided by Luo and Svendsen [69], who have developed a break-up model for bubbles or droplets in turbulent flow. Unlike its predecessors, this model contains no empirical fit parameters. In the model, a droplet of mass $M_{p}$ can break up into two daughter droplets of mass $M_{q}=M_{p} f_{B V}$ and $M_{r}=M_{p}\left(1-f_{B V}\right)$, where $f_{B V} \in[0,1]$ is the breakage mass fraction:

$$
f_{B V}=\frac{M_{q}}{M_{p}}=1-\frac{M_{r}}{M_{p}}
$$


due to the arrival of a turbulent eddy of size $\lambda$ at the surface of the droplet. The breakage rate of droplets with mass $M_{p}$ into smaller droplets, of which one has mass $M_{q}$, is given by integrating the product of the breakage probability $P\left(M_{p}: M_{q}, \lambda\right)$ and the droplet-eddy collision frequency $\dot{\omega}_{B, \lambda}\left(M_{p}\right)$ over all relevant eddy sizes.

$$
g\left(M_{p}: M_{q}\right) n_{p}=\int_{\lambda_{\min }}^{d_{p}} P\left(M_{p}: M_{q}, \lambda\right) \dot{\omega}_{B, \lambda}\left(M_{p}\right) d \lambda
$$

Here $\lambda_{\min }$ is the smallest eddy which may break-up the droplet. Note that eddies larger than the diameter $d_{p}$ of the droplet only transport the droplet and do not deform it. Similar to the droplet-droplet collisions, the droplet-eddy collision frequency $\dot{\omega}_{B, \lambda}\left(d_{p}\right)$ $\left(\left[\dot{\omega}_{B, \lambda}\left(d_{p}\right)\right]=1 / \mathrm{m}^{4} \mathrm{~s}\right)$ for $n_{p}$ droplets of diameter $d_{p}$ per volume with $n_{\lambda}$ eddies of sizes between $\lambda$ and $\lambda+d \lambda$ per volume is given by

$$
\dot{\omega}_{B, \lambda}\left(d_{p}\right)=\frac{\pi}{4}\left(d_{p}+\lambda\right)^{2} u_{\lambda}^{\prime} n_{\lambda} n_{p}
$$

Note that $\int n_{\lambda} d \lambda$ give the number of eddies per unit volume. Here $u_{\lambda}^{\prime}$ is the velocity of the eddies and this is also assumed to be the scale of the velocity difference between the eddy and the droplet. Note that here the eddies are smaller than the droplet, contrary to the case of coalescence in which case the eddies are larger than the droplet. The eddy velocity $u_{\lambda}^{\prime}$ is known in the inertial subrange for isotropic turbulent $[62,63]$. The eddy number density $n_{\lambda}$ is estimated using the energy spectrum in the inertial subrange. The result is, see [69]

$$
\dot{\omega}_{B, \lambda}\left(d_{p}\right)=0.923(1-\alpha) n_{p} \epsilon^{1 / 3} d_{p}^{2} \frac{\left(1+\lambda / d_{p}\right)^{2}}{\lambda^{11 / 3}}
$$

The break-up probability is equal to the chance that the arriving eddy contains more kinetic energy than the increase in interfacial energy required for the formation of two new droplets. Only the interfacial energy increase is incorporated in the criterion for break-up. A drawback of this model is that it does not take the disperse phase viscosity into account in the deformation of the droplets. In the current application the dispersed phase has a high viscosity. Luo and Svendsen [69] define $\chi$ as the ratio of the increase in interfacial energy and the kinetic energy of the eddy. The critical $\chi_{c}$, at which just sufficient energy is available for breakage, is defined as

$$
\chi_{c}=\frac{12\left(f_{B V}^{2 / 3}+\left(1-f_{B V}\right)^{2 / 3}-1\right) \sigma d_{p}^{2}}{2 \rho_{w} \epsilon^{2 / 3} \lambda^{11 / 3}}
$$

The probability is then given by

$$
P\left(M_{p}: M_{q}\right)=1-\int_{0}^{\chi_{c}} e^{-\chi} d_{p} \chi=e^{-\chi_{c}}
$$

Finally, the break-up rate of a droplet with mass $M_{p}$ into two droplet of mass $M_{q}$ and $M_{r}$, respectively, is given by

$$
g\left(M_{p}: M_{q}\right) n_{p}=0.923(1-\alpha) n_{p}\left(\frac{\epsilon}{d_{p}^{2}}\right)^{1 / 3} \int_{\lambda_{\min / d_{p}}}^{1} \frac{\left(1+\lambda / d_{p}\right)^{2}}{\left(\lambda / d_{p}\right)^{11 / 3}} e^{-\chi_{c}} d_{p}\left(\lambda / d_{p}\right)
$$


In addition to the assumption of isotropic turbulence and the neglect of the viscous effects of the dispersed phase, a third unfavorable characteristic of the model is that it predicts the generation of too many very small droplets compared to experimental data [54]. However, the widely-used Luo and Svendsen model is one of the more advanced break-up models currently available and is the only break-up model available in Ansys CFX 14.0. Therefore it has been used in the present study.

\section{Break-up results}

The simulation for two-phase flow which incorporated the break-up model by Luo and Svendsen had difficulty to converge the residuals, even for a very small time step. Also, counter-intuitive results were observed, such as larger increases in the mean droplet diameter when the break-up model was enabled compared to the results without break-up modeling. A simulation of the flow in a simplified channel was carried out in order to study the problem more thoroughly. In this test case the velocity, pressure, turbulent dissipation and turbulent kinetic energy were set to constant values throughout the domain. In addition, coalescence modeling was not enabled and only the population balance equations for break-up were solved. Analysis of the results showed unbalanced mass fluxes between the droplet size groups, such as, mass flow from a smaller size group into a larger one. This result is clearly unphysical.

Ansys support was contacted and it was confirmed that there was a problem with the numerical implementation of the Luo and Svendsen break-up model. The issue is investigated by Ansys and the problem is expected to be solved in future releases of Ansys CFX. Unfortunately, at this point a model for break-up can not be used.

However, some estimates for the break-up can still be made. The turbulent dissipation is high near the solid surfaces of the pipe wall and the ISE. However, inside the separator much lower values are seen. Further away than $1 \mathrm{~mm}$ from the pipe wall, the turbulent dissipation is less than $50 \mathrm{~m}^{2} / \mathrm{s}^{3}$. For the parameters listed in table 6.1, equations (6.40) and (6.42) give a maximum droplet diameter $d_{\max }=231$ and $325 \mu \mathrm{m}$, respectively. Here $d_{\max }$ for the correlation of Davies has been iteratively calculated using $\Delta u=\left(\epsilon d_{\max }\right)^{1 / 3}$. Close to the pipe wall the oil volume fraction is very small. Therefore the assumption is made that after the flow passes the ISE, where a considerable reduction of the mean droplet size occurs, no subsequent droplet break-up is experienced further downstream. Useful information can therefore be obtained from a poly-dispersed flow simulation without break-up modeling, if the inlet droplet diameter is small enough that negligible break-up occurs as the flow passes the ISE. In preliminary droplet size measurements at the TUD it was found that a $50 \mu \mathrm{m}$ diameter droplet is a good representation of the smallest droplets that are created by the break-up. Therefore, the droplet size at the inlet is set to $50 \mu \mathrm{m}$

\begin{tabular}{|ll||lll|}
\hline$\epsilon$ & $=50 \mathrm{~m}^{2} / \mathrm{s}^{3}$ & $\rho_{w}=1067.8 \mathrm{~kg} / \mathrm{m}^{3}$ \\
$\mu_{o}$ & $=19.4 \times 10^{-3}$ Pa.s & $\sigma$ & $=0.021 \mathrm{~N} / \mathrm{m}$ \\
\hline
\end{tabular}

Table 6.1: Input parameters used for calculation of maximum droplet diameter $d_{\max }$. 
in the poly-dispersed flow simulations. In this way, a worst-case scenario for very small incoming droplets is computed and increase in droplet size due to coalescence and the effect this has on the separation can be considered.

\subsection{Poly-dispersed two-phase flow}

\subsubsection{Poly-dispersed flow results}

In the poly-dispersed two-phase flow simulation all the oil droplets have a diameter of 50 $\mu \mathrm{m}$ at the inlet. The numerical results for two-phase flows with mono-dispersed $50 \mu \mathrm{m}$ droplets have been discussed in section 5.4.4. In this section the degree of droplet growth by coalescence and the consequences this has for the separation efficiency are examined. The computational mesh, time step of $\Delta t=0.5 \mathrm{~ms}$ and solver settings are equal to those used for the two-phase flow simulations in chapter 5 .

Ten size groups are employed to represent the droplet size distribution. A compromise has to be made in the number of size groups, between improving accuracy and increasing computational time. The mean diameters and mean masses of droplets in the size groups are given in table 6.2. The first few size groups approximately match the possible droplet sizes that can be generated by coalescence in order to obtain a good description of the behavior of these groups. As will be shown later, the results showed that $50 \%$ of the oil phase is contained in these size groups so their behavior should be predicted accurately. However, the size group resolution decreases as larger size groups consist out of wider ranges of droplet sizes. Full resolution is not attainable, for instance 50 size groups would already be required for the relatively narrow droplet diameter range from 50 to $180 \mu \mathrm{m}$. The large computational costs associated with the accurate description of wide droplet size distributions is the main drawback of the class method for population balances.

Schutz et al. [99] use 19 size groups in order to describe the evolution of the droplet size

\begin{tabular}{|c|c|c|}
\hline Size Group $p$ & $d_{p}(\mu \mathrm{m})$ & $m_{p} / m_{1}$ \\
\hline 1 & 50 & 1 \\
2 & 60 & 1.7 \\
3 & 70 & 2.7 \\
4 & 80 & 4.1 \\
5 & 90 & 5.8 \\
6 & 105 & 9.3 \\
7 & 120 & 13.8 \\
8 & 140 & 22.0 \\
9 & 160 & 32.8 \\
10 & 180 & 46.7 \\
\hline
\end{tabular}

Table 6.2: Mean diameter $d_{p}$ and relative mean mass $m_{p} / m_{1}$ of size groups used for the poly-dispersed two-phase flow simulation. Mass of smallest droplet $m_{1}=5.77 \times 10^{-11} \mathrm{~kg}$. 
distribution in a hydrocyclone for liquid-liquid separation. However, the droplet diameters for the two investigated cases are 21.5 to $280 \mu \mathrm{m}$ and 11.3 to $805 \mu \mathrm{m}$, which is an increase in mass between the smallest and largest droplets of about $2.2 \times 10^{3}$ and $3.6 \times 10^{5}$, respectively. Bannari et al. [6] compared the results for the simulations of the polydispersed flow in a bubble column using $7,11,15$ and 25 size groups, with experimental data and found reasonable agreement for 11 or more size groups. For 11 size groups the ratio between the smallest and largest diameter is a factor 10, therefore the range of droplet masses covers a factor 1000. Finally, Alopaeus et al. [2] considered a liquid-liquid stirred tank and found only small changes in the solution if more than 15 size groups are used. Depending on the impeller speed of the stirred tank the droplet diameter varied between 10 to $250 \mu \mathrm{m}$ and 10 to $400 \mu \mathrm{m}$. The number of size groups used in the present work relative to the droplet diameter range, that is the size group resolution, is somewhat finer but otherwise comparable to what has been found in the literature.

The current implementation of the population balance method in CFX 14.0 uses a single velocity distribution for the oil. This means that $\mathbf{u}_{d}$ in equation (6.1) is the same for all size groups. Since the relative motion of the oil phase depends on the droplet diameter, different droplet sizes will have different velocities. However, the spread in velocities between the size groups is not expected to be large since in the current case the range of droplet diameters is relatively small and the drag between the phases is high due to the high volume fraction. However, the assumption of a homogeneous velocity distribution for the oil phase is clearly an approximation. Computation of the velocity distribution for each size group would necessitate solving three momentum equations for each size group in addition to calculating the mass fluxes between the size groups. For the current simulation of poly-dispersed flow this would increase the computational cost fivefold.

The drag force per unit volume acting on the oil phase for the poly-disperse two-phase flow is obtained by summing the contributions for all size groups

$$
M_{o}=\sum_{q=1}^{q_{\max }} \frac{3}{4} \frac{C_{D, q}}{d_{q}} \rho_{w} \alpha f_{q}\left|\left\langle\mathbf{u}_{w}\right\rangle-\left\langle\mathbf{u}_{o}\right\rangle\right|\left(\left\langle\mathbf{u}_{w}\right\rangle-\left\langle\mathbf{u}_{o}\right\rangle\right)
$$

Here $f_{q}$ is the size group fraction of size group $q$ defined in equation (6.8). In CFX 14.0 the summation term is approximated by

$$
\sum_{q=1}^{q_{\max }} \frac{C_{D, q} f_{q}}{d_{q}} \approx C_{D} \sum_{q=1}^{q_{\max }} \frac{f_{q}}{d_{q}}=\frac{C_{D}}{d_{32}}
$$

This is only exact for multiphase flow for which $\mathrm{Re}_{\mathrm{d}}>1000$ since $C_{D}$ is then constant. The Sauter mean diameter $d_{32}$ is defined as [12]

$$
d_{32}=\frac{\sum_{q=1}^{q_{\max }} n_{q} d_{q}^{3}}{\sum_{q=1}^{q_{\max }} n_{q} d_{q}^{2}}
$$

Here $n_{q}$ is the number density of size group $q$. The equality is equation (6.51) can be shown by rewriting equation (6.52) using $n_{q} d_{q}^{3}=6 / \pi \alpha f_{q}$ and $\sum f_{q}=1$. For polydispersed flow the drag coefficient $C_{D}$ is evaluated at $d=d_{32}$. 

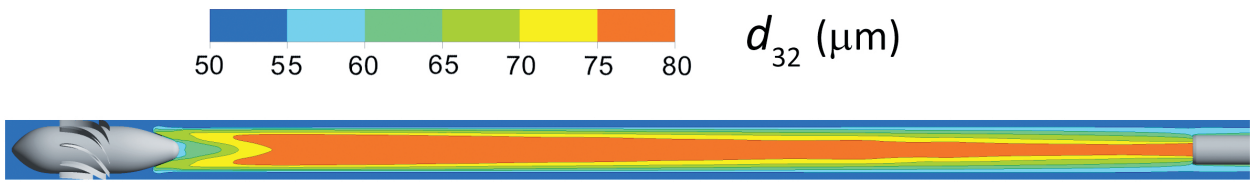

Figure 6.5: Distribution of the time-averaged Sauter mean diameter $d_{32}$ on a plane through the axis of the separator. Ten size groups are used, see table 6.2. Non-dimensional parameters are $\mathrm{FS}=0.30, \operatorname{Re}_{\mathrm{D}}=0.86 \times 10^{5}, \mathrm{Sw}=6.61$ and $\alpha_{\text {inlet }}=0.25$.

The velocity distribution is very similar to the one for the case of mono-dispersed twophase flow. As noted in section 5.4.4, the velocity distribution changes little with variation in droplet diameter. Therefore, the velocity distributions of the poly-dispersed flow simulation are not shown here. Figure 6.5 shows the distribution of the time-averaged Sauter mean diameter $d_{32}$ on a plane through the axis of the separator. The initial droplet size of $d_{32}=50 \mu \mathrm{m}$ increases to about 75 to $80 \mu \mathrm{m}$ in the center of the pipe. So although considerable coalescence is seen, the maximum Sauter diameter remains relatively small. The region of larger droplets in the center is wider in the upstream part of the separator where it covers part of the reversed flow region. While flowing towards the ISE, a small portion of this region with high $d_{32}$ is fed into the central positive axial velocity core. However, most is mixed with the outer part of the reversed flow region, where $d_{32}$ is

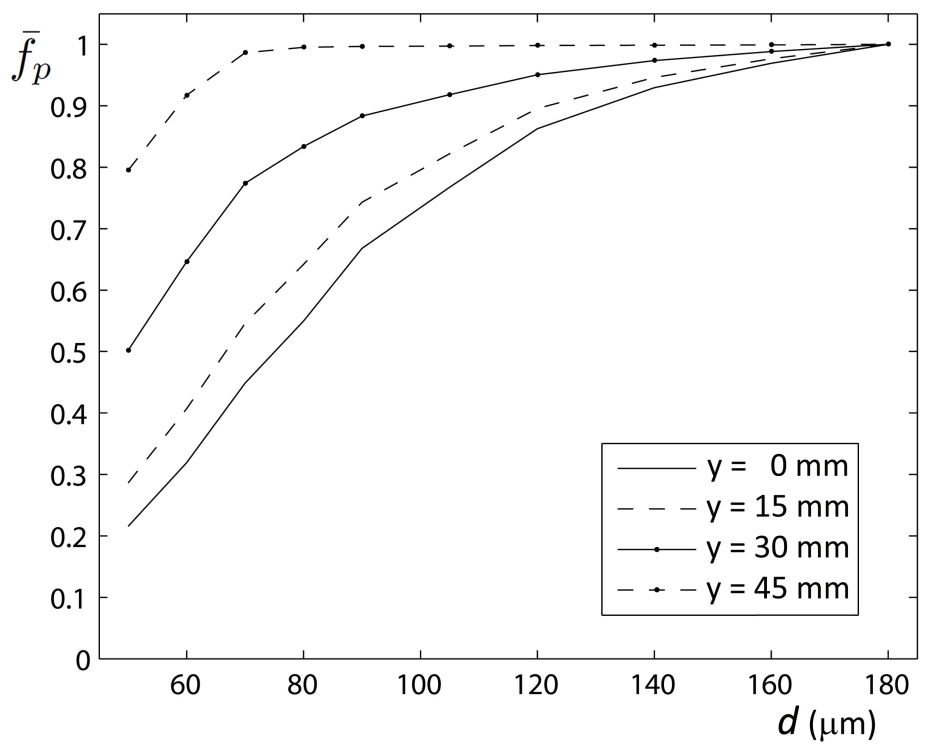

Figure 6.6: Cumulative size group fraction $\bar{f}_{p}$ for $y=[0,15,30,45] \mathrm{mm}$ at $z=1.5 \mathrm{~m}$. Ten size groups are used, see table 6.2. Non-dimensional parameters are $\mathrm{FS}=0.30, \operatorname{Re}_{\mathrm{D}}=0.86 \times 10^{5}, \mathrm{Sw}$ $=6.61$ and $\alpha_{\text {inlet }}=0.25$. 
relatively small. Just aft of the ISE a region with a smaller $d_{32}$ is seen. This region is mainly fed by the outer part of the reversed flow region with its low $d_{32}$. Also the low turbulent dissipation in this region leads to lower coalescence rates.

A more detailed view of the droplet size distribution can be obtained by examining the cumulative size group fraction

$$
\bar{f}_{p}=\sum_{q=1}^{p} f_{q}
$$

This quantity gives the fraction of the total oil volume present in the size groups up to size group $p$. Figure 6.6 shows the cumulative size group fraction at $y=0,15,30$ and $45 \mathrm{~mm}$ at axial station $z=1.50 \mathrm{~m}$. Near the wall, at $y=45 \mathrm{~mm}$, about $80 \%$ of the oil volume is still present in the smallest size group of $50 \mu \mathrm{m}$ droplets. Nearly all the oil is taken up by small droplet sizes from 50 to $70 \mu \mathrm{m}$. For a smaller radius, more oil volume has coalesced into the larger size groups, leading to a larger Sauter mean diameter as is shown in figure 6.5. The main reason for this increase in coalescence is the increasing volume fraction for smaller radius. Large changes can be seen in the cumulative size group fraction for larger distances from the wall. However, the difference between $y=0$ and $15 \mathrm{~mm}$ is relatively small. Further coalescence is limited since on the one hand the coalescence efficiency is much lower for pairs of larger droplets and on the other hand the number of small diameter droplets which easily coalesce is substantially reduced.

At the axis of the separator, the $50 \mu \mathrm{m}$ droplets make up about a fifth of the total oil

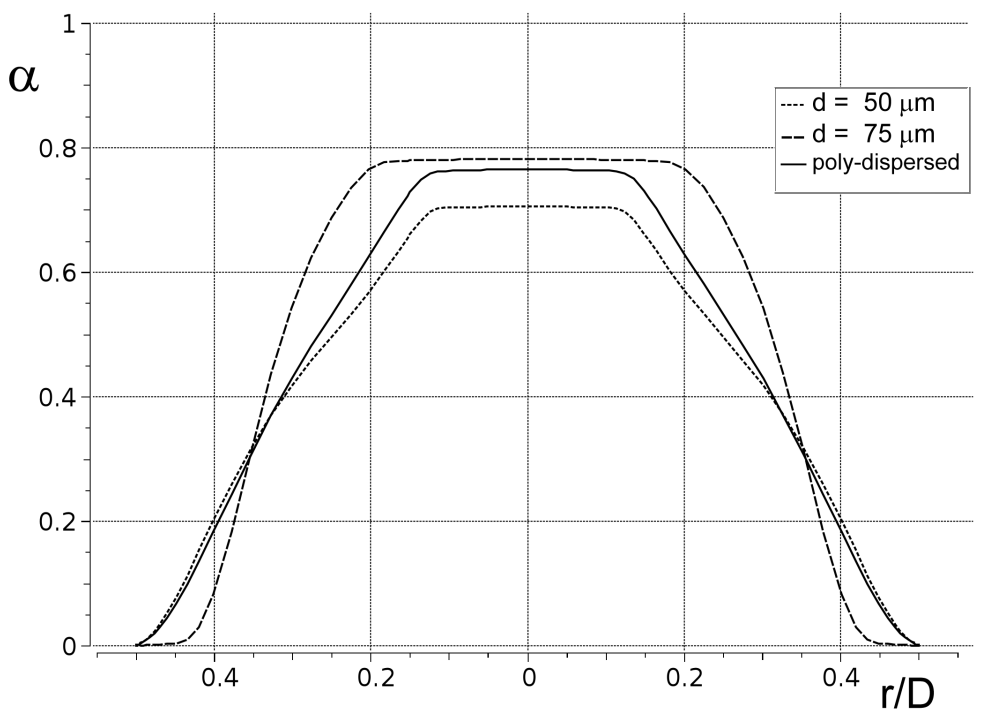

Figure 6.7: Radial distribution of the time-averaged oil volume fraction $\alpha$ on a line through the center of the separator in the cross-plane $z=1.50 \mathrm{~m}$ for mono-dispersed droplet diameters $d=50$ and $75 \mu \mathrm{m}$ and for poly-dispersed flow. Ten size groups are used, see table 6.2. Non-dimensional parameters are $\mathrm{FS}=0.30, \mathrm{Re}_{\mathrm{D}}=0.86 \times 10^{5}, \mathrm{Sw}=6.61$ and $\alpha_{\text {inlet }}=0.25$. 


\begin{tabular}{|c|c|c|c|c|c|c|c|}
\hline$d$ & $\alpha_{H P O}$ & $\alpha_{L P O}$ & hold up & $\eta_{1}$ & $\frac{\Delta Q_{o}}{Q_{o, \text { inlet }}}$ & $\eta_{2}$ & $\frac{1}{1-F S} \frac{\Delta Q_{o}}{Q_{o, \text { inlet }}}$ \\
$(\mu \mathrm{m})$ & $(\%)$ & $(\%)$ & $(\%)$ & $(\%)$ & $(\%)$ & $(\%)$ \\
\hline \hline 50 & 14 & 51 & 37 & 61 & 0.53 & 45 & 0.76 \\
poly-dispersed & 12 & 55 & 39 & 65 & -0.18 & 50 & -0.25 \\
75 & 7.6 & 65 & 41 & 79 & 0.38 & 70 & 0.54 \\
100 & 4.8 & 72 & 42 & 86 & 0.56 & 80 & 0.80 \\
\hline
\end{tabular}

Table 6.3: Separation efficiencies obtained from numerical simulations of mono and polydispersed flow. $\mathrm{FS}=0.30, \mathrm{Re}_{\mathrm{D}}=0.86 \times 10^{5}, \mathrm{Sw}=6.61$ and $\alpha_{\text {inlet }}=0.25$.

volume. The fractions of the size groups with diameter between 60 and $140 \mu \mathrm{m}$ all have a similar fraction of about $10 \%$. The groups with largest sizes contain each about $5 \%$ of the oil volume. The cumulative size fraction in the center of the pipe is very similar at upstream locations of $z=1.50 \mathrm{~m}$. This indicates that the maximum droplet diameter, to be obtained by turbulent-induced coalescence, is more or less reached at a small distance downstream of the ISE. Since the region of higher Sauter mean diameter is wider at upstream locations the distribution of the cumulative size group fractions at larger radius differs somewhat from the situation at $z=1.50 \mathrm{~m}$, however, the trends are the same.

Figure 6.7 compares the radial distribution of the time-averaged oil volume fraction on a line through the center of the separator in the cross-plane $z=1.50 \mathrm{~m}$ computed for the mono-dispersed flow with $d=50$ and $75 \mu \mathrm{m}$ and for the poly-dispersed flow. At $z=$ $1.50 \mathrm{~m}$, the Sauter mean diameter in the poly-dispersed case is approximately equal to the $75 \mu \mathrm{m}$ mono-dispersed case near the center $(r / D<0.15)$. However, the distribution of the oil volume fraction over the whole cross-plane appears very similar in shape to the $50 \mu \mathrm{m}$ droplet case. Of course the poly-dispersed results show higher values for the oil volume fraction since the droplet diameter is larger. So the results for the poly-dispersed flow simulation are closer to the results of the $50 \mu \mathrm{m}$ droplet case than to the $75 \mu \mathrm{m}$ droplet case.

The same conclusion can be drawn from the separation efficiencies. The separation efficiencies for the poly-dispersed flow simulation is given in table 6.3 along with the results for the droplet diameter variation study of section 5.4.4. As expected the separation efficiencies improve when droplet coalescence is accounted for. However, the gains are relatively small. Many small droplet are still present in the flow, leading to a substantial spill-over of oil towards the HPO.

The droplet size distribution and separation characteristics will be different for a different droplet size distribution at the inlet. Still, based on the current results it is expected that also for larger droplets at the inlet the positive effect of coalescence on the separation efficiencies is limited. For larger droplets the amount of coalescence is even less due to lower coalescence efficiencies and lower collision probability. On the other hand, droplet break-up, which is absent in the current simulation, is likely to play a larger role when the 
inlet droplet diameter is increased.

The mono-dispersed case for $50 \mu \mathrm{m}$ diameter droplets over-estimates the separation efficiency compared to the measurements at the TUD. So the current poly-dispersed flow simulation does not improve the comparison between experimental and numerical results. However, the main goal of calculating the poly-dispersed flow is to investigate the extent of droplet growth by coalescence and its effect on the separation efficiency for this application. The current approach, however, has its limitations.

\subsubsection{Limitations of population balance method}

One important limitation of the method employed above is that droplet break-up is absent. Droplet break-up is an important flow feature in these highly turbulent flows. Experimental data show significant reduction of the droplet diameter when the flow passes from the inlet through the ISE. Further break-up may be experienced in the region where the reversed flow region starts, near the walls and around the entrance of the pick-up tube. Even if the Luo and Svendsen break-up model [69] could be used, break-up of non-turbulent flow origin, for instance shear-induced break-up in regions with large velocity gradients, will still not be included. Due to the absence of the break-up model it is necessary to impose a rather artificial inlet droplet size distribution, which estimates the effects of downstream break-up. The inclusion of droplet break-up modeling in future work is highly recommended.

A second major shortcoming of the present modeling is that it does not accurately describe the coalescence process for high volume fractions. In the center of the pipe a dense emulsion layer is formed. Not only are the turbulence velocity fluctuations expected to be considerably damped, also the inter-droplet distance is so small that the collision mechanism is different from that in more dilute regions. Droplets are expected to be in prolonged contact with each other and they may roll-over and slide along each other. The contact time given in equation (6.27) will not longer hold. An oil core may form in the center of the pipe, which leads to a coalescence process which cannot be described by binary or droplet-droplet coalescence.

The coalescence behavior is a dense emulsion in turbulent flow is not well understood. Experimental research is difficult since there is no optical accessibility and non-optical measuring methods may drastically influence the flow. The investigation of the coalescence characteristics is ongoing, see for instance Krebs et al. [60]. Krebs investigated the growth of a pure oil layer in an emulsion under centrifugation. The results indicated that the coalescence rates of oil droplets with the pure oil layer is much higher than for dropletdroplet coalescence. One could approximate the coalescence process of a droplet with a pure oil core by the coalescence process of a small and very large droplet. However, the turbulence-induced coalescence model predicts the opposite trend, since the coalescence efficiency lowers for droplet pairs with larger equivalent radius. The equivalent radius is defined in equation (6.22). To further complicate the matter, the results of Krebs et al. [60] indicate that the presence of surfactants greatly influences the coalescence rate. This feature is also not included in the numerical method. More experimental data is required for the development of a population balance model for the binary coalescence of droplets 
in a dense emulsion under centrifugation. It is also not evident how these results change if turbulence is present in the flow.

A third limitation of the poly-dispersed flow simulations are the long computational times that are required. The calculation of 10 size fractions accounted for about $15 \%$ of the computational time, and its impact on the total computational time is therefore limited. However, the evolution of the size fractions is a very slow process. A wall clock time of about 3.5 month on a 24-CPU present-day computer cluster was necessary to compute the solution, including the transient statistics. These computational costs limit the applicability of time-dependent, poly-dispersed flow simulations for oil-water separator design in industry.

\subsection{Conclusion}

An analysis of the effect of droplet coalescence on the flow has been carried out using a poly-dispersed two-phase flow simulation. A method of classes is used to formulate the population balance equations. The coalescence model of Coulaloglou and Tavlarides [23] has been implemented in CFX 14.0 using user-defined functions. This model is developed for liquid-liquid dispersions and is therefore better suited for the current application than the standard CFX option of the Prince and Blanche model [84]. The Prince and Blanche model is intended for gas-liquid flows, such as bubble columns. The surface mobility of the gas-liquid interface leads to an overprediction of the coalescence rates for oil-water flows. The coalescence rates are now much more realistic with the newly implemented model.

Unfortunately, the droplet break-up model of Luo and Svendsen [69] leads to unphysical results for the current range of flow conditions and material properties. Improvements are being carried out by Ansys CFX in order to fix this problem in future releases. Droplet break-up is an important feature of the flow and it is recommended to incorporate it into future work. In the separator the droplet break-up is expected to take place around the ISE and near the walls. Further downstream the dissipation rate is lower and the formation of relatively large droplets is possible. In preliminary measurements of the droplet size distribution, a representative size for the smallest droplets produced by break-up was found to be $d=50 \mu \mathrm{m}$. That is, these droplet will not be further broken up in the flow near the ISE. Also, as the fluid near the walls is devoid of oil, little break-up is expected there. Thus for an incoming flow with droplets of $50 \mu \mathrm{m}$ diameter, break-up is expected to be not of importance and a relevant case study could be done without break-up modeling. The aim of this case study is to examine the extent of droplet growth by coalescence and its effect on the separation efficiency.

Considerable growth of the incoming droplets is indeed seen in the numerical simulation, although the maximum Sauter mean diameter is rather limited to about 75 to $80 \mu \mathrm{m}$. This is caused by the lower coalescence efficiencies seen for larger droplets, since they are more easily deformed. This indicates that turbulence-induced coalescence does not generate large droplets in the in-line separator. In terms of the distribution of the oil volume fraction and the separation efficiencies, the results for poly-dispersed flow are 
in-between the results for mono-dispersed flow with 50 and $75 \mu \mathrm{m}$ droplets, although it is closer to the $50 \mu \mathrm{m}$ result. The separation efficiencies improve by about $5 \%$ when coalescence in enabled.

The use of the coalescence model leads to serious errors in the dense emulsion in the center of the pipe. The turbulent collisions may very well not be present, or at least its frequency is strongly reduced. A model for other mechanisms of coalescence is absent. Such a model could take into account the prolonged contact between droplets as they slide along each other, the high volume fraction and the compression due to the centrifugal force acting on the droplets. The predicted droplet size distribution in the center should therefore be viewed in a qualitative manner only, as the quantitative results contain substantial errors. It is expected that due to the longer contact times, the coalescence rate will be higher in the center than predicted by the current method. However, this growth takes place in the center of the pipe. The oil in this region is already separated and will exit the separator through the LPO. Therefore, the increase in separation efficiency when coalescence mechanisms in the dense emulsion are incorporated may be fairly small after all. However, for a better prediction of the droplet size distribution in the center of the pipe the current coalescence model is incomplete and further development of the coalescence model must be undertaken. 
CHAPTER 7

\section{Concluding remarks and recommendations}

A design study has been carried out to develop an oil-water separator. In the study, a number of alternative configurations has been explored. Analysis using CFD showed comparable performance for these initial designs. Because of its in-line geometry, and its relatively low pressure drop, the separator featuring an internal swirl element (ISE) has been adopted for further research. The in-line aspect facilitates implementation in existing pipe lines and allows for a compact design. The final design features a straight pipe with as internals the ISE and a straight concentric pick-up tube. For a pipe with an internal diameter of $0.10 \mathrm{~m}$, the distance between the afterbody of the ISE and the entrance of the pick-up tube has been chosen as $1.7 \mathrm{~m}$. This choice has been based on results of experiments. A method for designing future separators maybe derived from the present design process.

The current separator is a first design and improvement to its geometry can be made. The tail of the ISE is rather blunt in order to decrease the total length of the ISE. Just downstream of the ISE a dead water zone forms, which takes up space inside the separator. Furthermore, it induces unsteady flow effects. The length and unsteadiness of the dead water zone may be reduced by using a more conical, pointed tail shape. The pick-up tube is currently a simple straight pipe. Large increases in velocity are seen near its entrance, which leads to pressure drop and turbulence production. Also the pipe wall can be slightly tapered in order to compensate for the frictional losses in the azimuthal velocity. It is recommended to experiment with design variations in order to search for a possibly more optimum separator.

The generation of the strongly swirling flow leads to a W-shaped axial velocity distribution with an annular reversed flow region. This flow pattern cannot merely be explained by an adverse pressure gradient, which in turn is caused by friction at the pipe wall. A straightforward, cause-and-effect explanation of the flow pattern has yet to be found. The 
features in this flow are expected to be caused by mechanisms similar to those associated with vortex break-down. The W-shaped axial velocity distribution is also seen in the LDA experiments carried out at the TUD.

The change in flow pattern for increasing flow rate shows a predictable trend. In terms of non-dimensional variables, the results collapse onto a single velocity distribution for low-viscosity fluids.

For variations in other flow parameters the change in the velocity distribution is harder to anticipate. An increase in flow split FS shows a substantial increase in the axial and azimuthal velocity near the center of the pipe, while at larger radii the flow does not appear to be affected. In general, changes in boundary conditions in the center, such as changes in flow split or modifications of the geometry of the pick-up tube, have a large influence on the flow field. On the other hand, features in the region closer to the pipe wall, such as the flow straightener, barely have an impact in the flow upstream. An increase of the flow split leads to a large increase of the centrifugal force, locally an increase of $200 \%$ is possible within the operational range of the flow split. For increasing flow split both numerical and experimental results show an increase in separation efficiency. However, at a certain FS the separation efficiency levels off and a maximum is reached. A further increase of FS will then only lead to more pressure drop over the separator.

A change in swirl angle $\mathrm{Sw}$ leads to large changes in the flow. When the swirl angle is considerably reduced a V-shaped axial velocity distribution develops. The study on the variation of the swirl angle showed that for lower swirl angles, a smaller annular reversed flow region is formed. It is therefore recommended to investigate in-line separators with lower swirl angle in future research, since the reversed flow region is not beneficial to separation. Most of the oil that is captured by the annular reversed flow region is recirculated to the outer flow at upstream locations near the ISE. The reversed flow region occupies volume which leads to a less compact separator. Although sufficient centrifugal forces on the droplets should be present, a decrease in swirl angle may lead to improved separation efficiency. The flow field generated by an ISE design with lower swirl angle can be investigated numerically by imposing a distribution of the velocity on a cross-flow plane just aft of the location of the vanes. It has been shown that this is an accurate approximation. In this way design criteria for the vane geometry can be derived. In addition, a lower swirl angle will lead to a lower required pressure drop over the ISE.

The static pressure varies considerably over the separator. For the nominal flow rate of $56.5 \mathrm{~m}^{3} / \mathrm{hr}$, the pressure drop over the ISE is $\Delta\langle\bar{p}\rangle=1.8 \mathrm{bar}$, while the pressure differences between inlet and HPO and between inlet and LPO are 1.6 and 2.5 bar, respectively. The pressure drop between the inlet and the LPO scales as $\Delta\langle\bar{p}\rangle \propto u_{z, b}^{2.085}$. Therefore, the non-dimensional pressure drop between inlet and LPO for strongly swirling flow increases with higher $\mathrm{Re}_{\mathrm{D}}$. This is opposite to the trend observed for non-swirling pipe flow. The pressure drop between inlet and LPO also increases with increasing flow split, although the dependence is weak: $\mathrm{Eu} \propto \mathrm{FS}^{0.17}$. As high frictional losses are experienced by the flow, gains from pressure recovery by de-swirling the flow are small.

A numerical simulation for poly-dispersed flow, using a turbulence-induced coalescence model without droplet break-up for liquid-liquid two-phase flows, predicted an increase in droplet diameter from $50 \mu \mathrm{m}$ to slightly over $75 \mu \mathrm{m}$ in the center of the pipe. This 
indicates that turbulence-induced coalescence does not generate very large droplets in the in-line separator. Smaller droplets are found in the outer part of the pipe. The low oil volume fraction in that region leads to fewer droplet collisions and therefore prevents further droplet growth. The numerical simulation for poly-dispersed flow showed improved separation performance over the results for the mono-dispersed case with droplets of 50 $\mu \mathrm{m}$ diameter. However, the improvement of $5 \%$ is modest.

While the numerical results for single-phase flow have shown reasonable agreement with the experimental results, the predictions for two-phase flow show large deviations from the results of the measurements. The separation efficiency is overpredicted by 25 to $50 \%$ depending on the flow conditions and on the used definition of separation efficiency. For high inlet volume fractions, the flow simulations show a decreasing separation efficiency due to a flooding phenomenon. In contrast, the experimental results nearly collapse onto a single curve when the separation efficiency is plotted as function of the flow split divided by the inlet oil volume fraction: FS/ $\alpha_{\text {inlet }}$. Therefore, in terms of quantitative predictions of the distribution of the oil volume fraction and separation efficiency, the numerical flow simulations are not close to the data measured in the flow rig at TU Delft.

There may be various causes for this discrepancy between numerical and experimental results. A major reason is the use of a too large droplet size at the inlet in the numerical solutions. For separation the droplet size is the prime parameter. The difference between the numerically and experimentally determined separation efficiencies becomes smaller when smaller, more representative droplet sizes are used in the computations. The use of the correct droplet size (distribution) at the inlet is an essential prerequisite for an accurate prediction. It is a necessary but not sufficient condition as the results of the flow simulations in this study have shown. The evolution of the droplet size distribution for these flows is not captured by a constant mono-dispersed droplet size. Droplet break-up could unfortunately not be incorporated into the present analysis. It is recommended to incorporate droplet break-up models into future simulations for poly-dispersed flow. It is also important to validate these models in order to check whether droplet break-up, in for instance the vane section, is indeed accurately described.

The turbulence-induced coalescence model is unlikely to predict the correct droplet growth when the oil volume fraction increases to high values. In the current study the predicted droplet size in the center of the pipe is not accurate, and probably underpredicted. For an improved prediction of the droplet size distribution other mechanisms of coalescence should be included in the modeling. In these models the relation between the coalescence rate of a given pair of droplet sizes and the oil volume fraction as well as the centrifugal force should be specified. In addition, turbulent dissipation or the mean velocity and its gradients should be incorporated in order to provide a time-scale in which the coalescence must take place. If possible chemical aspects, such as salinity and the presence of surfaceactive components should be accounted for as well. Further experimental research is required to obtain more information on the behavior of very dense emulsions in turbulent flow subjected to centrifugation. This data is necessary to develop and to validate the improved coalescence models.

Data on the behavior of the dense emulsions can also be used to refine the existing drag laws. As comparison to micro-centrifuge experiments have shown that the Ishii-Zuber 
drag correlation overestimates the volume fraction even for relatively simple flow. Large changes in droplet size and possible phase inversion change the interfacial drag between the phases in ways not accounted for by the current drag laws. The flooding effect seen in the numerical flow simulations may be attributed to this incomplete description of the physics of the flow of the oil-water mixture.

In one of the simulations, a mono-dispersed droplet diameter of $50 \mu \mathrm{m}$ has been used. The separation efficiencies for this case are higher than found in the measurements for the same flow parameters, while the Sauter mean diameter in the experiments is larger than $50 \mu \mathrm{m}$. This indicates that, although the correct estimation of the inlet droplet size is important, more factors play a role. Turbulent dispersion is currently not included in the modeling. The motion of the dispersed phase is influenced by the instantaneous velocity of the continuous phase. While the turbulence modeling accounts for the effect of the turbulence on the mean velocity field, the turbulent dispersion should account for the effect of the turbulent fluctuations on the motion of the dispersed phase. The mean radial velocity can be zero at certain locations, meaning that the in and outward turbulent fluctuations cancel. However, if a gradient in oil volume fraction is present the turbulent eddies transport a net flux of oil from a region with a higher oil volume fraction to a region with a lower oil volume fraction. This mechanism could provide an explanation of the difference in separation efficiency predicted by the simulations and the efficiency found in the measurements. The turbulent flow in the separator is anisotropic and this should be reflected in the turbulent dispersion modeling. It is therefore recommended to implement such a turbulent dispersion model for these flows in order to investigate its effect on the separation performance. This analysis should show whether or not turbulent dispersion enhances the modeling of the real flow.

Turbulent two-phase flow is intrinsically complex and the modeling of such a flow can be made more accurate by using more sophisticated computational methods. These methods resolve more details of the physics of the flow rather than model these effects. However, these methods, such as volume of fluid methods, substantially increase the computational costs. Simulations employing these methods will lead to computational times that are too long for these methods to be used in the design process. Therefore, it is recommended to invest into improving the two-fluid RANS method in order to increase the accuracy of the predictions for two-phase flow in separators. Nevertheless, the computational times for the numerical methods used in the present study are already problematic and the results for two-phase flow show substantial discrepancies with experimental data. Therefore, further research is necessary before the use of transient numerical simulations is feasible in the optimization of the design of oil-water separators in an industrial environment. 


\section{Bibliography}

[1] I.H. Abbott and A.E. von Doenhoff. Theory of Wing sections. Dover Publications, inc., 1959.

[2] V. Alopaeus, J. Koskinen, and K.I. Keskinen. Simulation of the population balances for liquid-liquid systems in a non-ideal stirred tank. Part 1 - Description and qualitative validation of the model. Chemical Engineering Science, 54:5887-5899, 1999.

[3] Ansys, Inc. Ansys CFX 14.0 manual, 2012.

[4] Ansys, Inc. Ansys Fluent 14.0 manual, 2012.

[5] S. Arirachakaran, K.D. Oglesby, M.S. Malinowsky, O. Shoham, and J.P. Brill. An analysis of oil/water flow phenomena in horizontal pipes. In SPE Production Operations Symposium, number SPE 18836, Oklahoma, 1989. Society of Petroleum Engineers.

[6] R. Bannari, F. Kerdouss, B. Selma, A. Bannari, and P. Proulx. Three-dimensional mathematical modeling of dispersed two-phase flow using class method of population balance in bubble columns. Computers and Chemical Engineering, 32(12):3224-3237, 2008.

[7] G.K. Batchelor. Pressure fluctuations in isotropic turbulence. Mathematical Proceedings of the Cambridge Philosophical Society, 47:359-374, 1951.

[8] R.J. Belt. On the liquid film in inclined annular flow. PhD thesis, Delft University of Technology, 2007.

[9] T.B. Benjamin. Theory of the vortex breakdown phenomenon. Journal of Fluid Mechanics, 14:593-629, 1962. 
[10] J. Blazek, editor. Computational Fluid Dynamics: Principles and Applications. Elsevier, 2005.

[11] D. Bradley. The Hydrocyclone. Pergamon Press, 1965.

[12] C.E. Brennen. Fundamentals of Multiphase Flow. Cambridge University Press, 2005. ISBN-13 978-0-521-84804-6.

[13] $\mathrm{CH}$. Brücker. Some observations of vortex breakdown in a confined flow with solid body rotation. Flow, Turbulence and Combustion, 69:63-78, 2002.

[14] A.D. Burns, T. Frank, I. Hamill, and J.M. Shi. The Favre Averaged Drag Model for Turbulent Dispersion in Eulerian Multi-Phase Flows. In Proceedings of the 5th International Conference on Multiphase Flow, page paper no. 392, Yokohama, Japan, 2004.

[15] J.M. Campbell. Gas conditioning and processing, volume 2. John M. Campbell and Company, 2004.

[16] F. Chang and V.K. Dhir. Turbulent flow field in tangentially injected swirl flows in tubes. International Journal of Heat and Fluid Flow, 15(5):346-356, 1994.

[17] S. Chapman and T.G. Cowling. The Mathematical Theory of Non-Uniform Gases. Cambridge University Press, $3^{\text {th }}$ edition, 1970.

[18] J.C. Chen and C.A. Lin. Computations of strongly swirling flows with secondmoment closures. International Journal for Numerical Methods in Fluids, 30:493508, 1999.

[19] A.K. Chesters. The Modeling of Coalescence Processes in Fluid-Liquid dispersions: A Review of Current Understanding. Chemical Engineering Research and Design, 69:259-270, 1991.

[20] D. Cokljat, M. Slack, S.A. Vasquez, A. Bakker, and G. Montante. Reynolds-Stress Model for Eulerian multiphase. Progress in Computational Fluid Dynamics, 6(13):168-178, 2006.

[21] D.A. Colman. The Hydrocyclone for Separating Light Dispersions. PhD thesis, University of Southampton, 1981.

[22] J.J. Conti. International Energy Outlook 2010. Technical report, U.S. Energy Information Administration, July 2010. http://www.eia.gov/oiaf/ieo/index.html.

[23] C.A. Coulaloglou and L.L. Tavlarides. Description of interaction processes in agitated liquid-liquid dispersions. Chemical Engineering Science, 32:1289-1297, 1977.

[24] C.T. Crowe, M. Sommerfeld, and Y. Tsuji. Multiphase Flows with Droplets and Particles. CRC Press, 1997. ISBN 0849394694. 
[25] J.C. Cullivan, R.A. Williams, and C.R. Cross. Understanding the hydrocyclone separator through computational fluid dynamics. Transactions Institution of Chemical Engineers A, 81:455-466, 2003.

[26] J.T. Davies. Drop sizes of emulsions related to turbulent energy dissipation rates. Chemical Engineering Science, 40(5):839-842, 1985.

[27] R. Delfos, S. Murphy, D. Stanbridge, Z. Olujic, and P.J. Jansens. A design tool for optimising axial liquid-liquid hydrocyclones. Minerals Engineering, 17(5):721-731, 2004.

[28] J.J. Derksen. Separation Performance Predictions of a Stairmand High-Efficiency Cyclone. American Institute of Chemical Engineers Journal, 49(6):1359-1371, 2003.

[29] M. Dirkzwager. A New Axial Cyclone Design for Fluid-Fluid Separation. PhD thesis, Delft University of Technology, 1996.

[30] D.A. Drew. Mathematical modeling of two-phase flow. Annual Review of Fluid Mechanics, 15:261-291, 1983.

[31] D.A. Drew and S.L. Passman. Theory of Multicomponent Fluids. Springer-Verlag, 1999.

[32] D.F. van Eijkeren and H.W.M. Hoeijmakers. History force and drag correlation in a Lagrangian method applied to oil-water separation. In Proceedings of the 8th International Conference on CFD in Oil and Gas, Metallurgical and Process Industries, pages 111-1-111-10, Trondheim, Norway, 2011. Sintef.

[33] A. Einstein. Eine neue Bestimmung der Molekuldimensionen. Annalen der Physik, 19:289-306, 1906.

[34] A. Einstein. Berichtigung zu meiner Arbeit: Eine neue Bestimmung der Molekuldimensionen. Annalen der Physik, 34:591-592, 1911.

[35] S.E. Elghobashi and T.W. Abou-Arab. A two-equation turbulence model for twophase flows. Physics of Fluids, 26:931-938, 1983.

[36] U.S. Environmental Protection Agency. Regulating petroleum industry wastewater discharges in the united states and norway, January 2011. Information sheet.

[37] T. Ferreira and W. Rasband. ImageJ User Guide, IJ 1.46r edition, 2012.

[38] R.W. Fox and A.T. McDonald. Introduction to fluid dynamics. Wiley \& Sons, inc., 1973.

[39] C. Gomez, J. Caldentey, S. Wang, L. Gomez, R. Mohan, and O. Shoham. Oil/water Separation in Liquid/Liquid Hydrocyclones (LLHC): Part 1 - experimental investigation. SPE Journal, 7(4):353-372, 2002. 
[40] C.H. Gomez. Oil-Water Separation in Liquid-Liquid Hydrocyclones (LLHC) - EXperiment and Modeling. PhD thesis, University of Tulsa, 2001.

[41] A.D. Gosman and E. loannides. Aspects of Computer Simulation of Liquid-Fueled Combustors. Journal of Energy, 7(6):482-490, 1983.

[42] A.D. Gosman, C. Lekakou, S. Politis, R.I. Issa, and M.K. Looney. Multidimensional modeling of turbulent two-phase flows in stirred vessels. AIChE Journal, 38:19461956, 1992.

[43] W.G. Gray and P.C.Y. Lee. On the Theorems for Local Volume Averaging of Multiphase Systems. International Journal of Multiphase Flow, 3:333-340, 1977.

[44] A. Griggs, A. Zinchenko, and R. Davis. Gravity-driven motion of a deformable drop or bubble near an inclined plane at low reynolds number. International Journal of Multiphase Flow, 34:408-418, 2008.

[45] K. Hanjalic. Second-Moment Turbulence Closures for CFD: Needs and Prospects. International Journal of Computational Fluid Dynamics, 12(1):67-97, 1999.

[46] K. Hanjalic and B.E. Launder. A Reynolds stress model of turbulence and its application to thin shear flows. Journal of Fluid Mechanics, 52:609-638, 1972.

[47] J.O. Hinze. Fundamentals of the Hydrodynamic Mechanism of Splitting in Dispersions Processes. AlChE Journal, 1(3):289-295, 1955.

[48] A.J. Hoekstra. Gas flow field and collection efficiency of cyclone separators. PhD thesis, Delft University of Technology, 2000. ISBN 90-90143341-3.

[49] L. Y. Hu, L. X. Zhou, J. Zhang, and M. X. Shi. Studies on strongly swirling flows in the full space of a volute cyclone separator. AlChE Journal, 51(3):740 - 749, 2005.

[50] S. Huang. Numerical Simulation of Oil-Water Hydrocyclone Using Reynolds-Stress Model for Eulerian Multiphase Flows. Canadian Journal of Chemical Engineering, 83(5):829 - 834, 2008.

[51] H.M. Hulburt and S. Katz. Some problems in particle technology: A statistical mechanical formulation. Chemical Engineering Science, 19(8):555-574, 1964.

[52] M. Ishii and Hibiki T. Thermo-Fluid Dynamics of Two-Phase Flow. Springer-Verlag, 2005.

[53] M. Ishii and M. Zuber. Drag coefficient and relative velocity in bubbly, droplet or particulate flows. AlChE Journal, 25, 1979.

[54] H.A. Jakobsen. Chemical Reactor Modeling: Multiphase Reactive Flows, chapter The Population Balance Equation. Springer-Verlag Berlin Heidelberg, 2008. 
[55] F. Kaya and I. Karagoz. Performance analysis of numerical schemes in highly swirling turbulent flows in cyclones. Current Science, 94(10):1273 - 1278, 2008.

[56] A.C. King, J. Billingham, and S.R. Otto. Differential Equations. Cambridge University Press, 2003.

[57] O. Kitoh. Experimental study of turbulent swirling flow in a straight pipe. Journal of Fluid Mechanics, 225:445-479, 1991.

[58] A.N. Kolmogorov. The Local Structure of Turbulence in Incompressible Viscous Fluid for Very Large Reynolds Numbers. Proc. R. Soc. Lond., 434(1890), 1991.

[59] T. Krebs, C.P.G.H. Schröen, and R.M. Boom. A microfluidic method to study demulsification kinetics. Lab on a Chip, 12:1060-1070, 2012.

[60] T. Krebs, C.P.G.H. Schröen, and R.M. Boom. Separation kinetics of an oil-in-water emulsion under enhanced gravity. Chemical Engineering Science, 71:118-125, 2012.

[61] T. Krebs, J.J. Slot, C.P.G.H. Schröen, H.W.M. Hoeijmakers, and R.M. Boom. Emulsion Compression and Coalescence under Enhanced Gravity Studied with InSitu Microscopy. In Proceedings of the 9th International Conference on Advances in Fluid Mechanics, Split, Croatia, June 2012.

[62] R. Kuboi, I. Komasawa, and R. Otake. Behavior of dispersed particles in turbulent liquid flow. Journal of Chemical Engineering of Japan, 5:349, 1972.

[63] R. Kuboi, I. Komasawa, and R. Otake. Collision and coalescence of dispersed drops in turbulent liquid flow. Journal of Chemical Engineering of Japan, 5:423, 1972.

[64] D. Lathouwers. Modelling and simulation of turbulent bubbly flow. PhD thesis, Delft University of Technology, 1999.

[65] S Leibovich. The structure of vortex breakdown. Annual Review of Fluid Mechanics, 10:221-246, 1978.

[66] S. Leibovich and K. Stewartson. A sufficient condition for the instability of columnar vortices. Journal of Fluid Mechanics, 126:335-356, 1983.

[67] R.I. Lewis. Turbomachinery Performance Analysis. John Wiley \& Sons, 1996.

[68] Y. Liao and D. Lucas. A literature review on mechanisms and models for the coalescence process of fluid particles. Chemical Engineering Science, 65(10):2851 $-2864,2010$.

[69] H. Luo and H. F. Svendsen. Theoretical Model for Drop and Bubble Breakup in Turbulent Dispersions. AlChE Journal, 42:1225-1233, 1996.

[70] S. Majumdar. Role of Underrelaxation in Momentum Interpolation for Calculation of Flow with Nonstaggered Grids. Numerical Heat Transfer, 13:125-132, 1988. 
[71] M. Manninen, V. Taivassalo, and S. Kallio. On the mixture model for multi-phase flow. Technical report, Technical Research Center of Finland, 1996. VTT Publication 288.

[72] N.N. Mansour, J. Kim, and P. Moin. Reynolds-stress and dissipation-rate budgets in a turbulent channel flow. Journal of Fluid Mechanics, 194:15-44, 1988.

[73] D.L. Marchisio and R.O. Fox. Solution of population balance equations using the direct quadrature method of moments. Aerosol Science, 36:43-73, 2005.

[74] W. Matek, D. Muhs, H. Wittel, M. Becker, and D. Janasch. Machineonderdelen; Tabellenboek. Academic services, 2000. ISBN $=9039514224$.

[75] T.W. Mattner, P. N. Joubert, and M. S. Chong. Vortical flow. Part 1. Flow through a constant-diameter pipe. Journal of Fluid Mechanics, 463:259-291, 2002.

[76] R. McGraw. Description of aerosol dynamics by the quadrature method of moments. Aerosol Science and Technology, 27:255-265, 1997.

[77] R. Mei and J.F. Klausner. Shear lift force on spherical bubbles. International Journal of Heat and Fluid Flow, 15(1):62 - 65, 1994.

[78] S. Murphy, R. Delfos, M.J.B.M. Pourquie, Z. Olujic, P.J. Jansens, and F.T.M. Nieuwstadt. Prediction of strongly swirling flow within an axial hydrocyclone using two commercial CFD codes. Chemical engineering science, 62:1619-1635, 2007.

[79] S. Noroozi and S.H. Hashemabadi. CFD simulation of inlet design effect on deoiling hydrocyclone separation efficiency. Chemical Engineering and Technology, 32(12):1885-1893, 2009.

[80] Lucca-Negro O. and T. O'Doherty. Vortex breakdown: a review. Progress in Energy and Combustion Science, 27:431-481, 2001.

[81] E. Paladino, J. Aguirre, and E. Quintella. Developing multiphase models for liquidliquid hydrocyclone flow. In 6th International Conference on Multiphase Flow, page S6 Wed C 37, 2007.

[82] S. B. Pope. Turbulent Flows. Cambridge University Press, 2000.

[83] H.M. Prasser, A. Bottger, and J. Zschau. A new electrode-mesh tomograph for gas-liquid flows. Flow Measurement and Instrumentation, 9(2):111 - 119, 1998.

[84] M.J. Prince and H.W. Blanch. Bubble Coalescence and Break-up in Air-Sparged Bubble Columns. AIChE Journal, 36:1485-1499, 1990.

[85] A. Prosperetti and G. Tryggvason. Computational Methods for Multiphase Flow, chapter Averaged equations for multiphase flow, pages 237-282. Cambridge University Press, 2007. 
[86] D. Qin, Y. Xia, and G. Whitesides. Soft lithography for micro- and nanoscale patterning. Nature Protocols, 5:491-502, 2010.

[87] M.J. Raw. Robustness of Coupled Algebraic Multigrid for the Navier-Stokes Equations. In 34th Aerospace and Sciences Meeting \& Exhibit, AIAA 96-0297, 1996.

[88] R. Roscoe. The viscosity of suspensions of rigid spheres. British Journal of Applied Physics, 3(8):267-269, 1952.

[89] J.C. Rotta. Statistische theorie nichthomogener turbulenz. Zeitschrift fur Physik, 124:547-572, 1951.

[90] C.A. Rubio, J.M. Doval, and J.A. Medina. Rubiales Oil Field: A Heavy-Oil Colombian Example of Excellent Operative PCP Systems Development. In Latin American and Caribbean Petroleum Engineering Conference proceedings, 15-18 April 2007.

[91] Z. Rusak, S. Wang, and Whiting C.H. The evolution of a perturbed vortex in a pipe to axisymmetric vortex breakdown. Journal of Fluid Mechanics, 366:211-237, 1998.

[92] H. Rusche. Computational Fluid Dynamics of Dispersed Two-Phase Flows at High Phase Fractions. PhD thesis, Imperial College, 2002.

[93] P. G. Saffman. The lift on a small sphere in a slow shear flow. Journal of Fluid Mechanics, 22(2):385-400, 1965.

[94] P. G. Saffman. The lift on a small sphere in a slow shear flow - corrigendum. Journal of Fluid Mechanics, 31(3):624-624, 1968.

[95] C. Sandau, J. Waddell, and T. Berthelet. Novel Approach to High Water Cut Measurement in a Mature Oil Field. In Abu Dhabi International Petroleum Exhibition and Conference proceedings, 3-6 November 2008.

[96] J. Sanyal, D.L. Marchisio, R.O. Fox, and K. Dhanasekharan. On the Comparison between Population Balance Models for CFD Simulation of Bubble Columns. Industrial \& Engineering Chemistry Research, 44(14):5063-5072, 2005.

[97] L. Schiller and A. Naumann. Uber die grundlegenden berechnungen bei der schwerkraft aufbereitung. Zeitschrift des Vereines Deutscher Ingenieure, 77:318-320, 1933.

[98] R. Schook. Hydrocyclone. FMC Technologies, February 2007. Patent, International publication no. WO 2007/021181.

[99] S. Schutz, G. Gorbach, and M. Piesche. Modeling fluid behavior and droplet interactions during liquid-liquid separation in hydrocyclones. Chemical Engineering Science, 64(18):3935-3952, 2009. 
[100] B. Selma, B. Bannari, and P. Proulx. Simulation of bubbly flows: Comparison between direct quadrature method of moments (DQMOM) and method of classes (CM). Chemical Engineering Science, 65(6):1925 - 1941, 2010.

[101] C.C. Shir. A Preliminary Numerical Study of Atmospheric Turbulent Flows in the Idealized Planetary Boundary Layer. Journal of Atmospheric Sciences, 30:13271339, 1973.

[102] A. Silset. Emulsions (w/o and o/w) of Heavy Crude Oils. Characterization, Stabilization, Destabilization and Produced Water Quality. PhD thesis, Norwegian University of Science and Technology, 2008.

[103] C. Simonin and P.L. Violett. Predictions of an Oxygen Droplet Pulverization in a Compressible Subsonic Coflowing Hydrogen Flow. In Numerical Methods for Multiphase Flows. FED91, pages 65-82, 1990.

[104] J.J. Slot, L.J.A.M. van Campen, H.W.M Hoeijmakers, and R.F. Mudde. Separation of oil droplets in swirling water flow. In Proceedings of the $7^{\text {th }}$ International Conference on Multiphase Flow, pages 1-10, Tampa, Florida, June 2010.

[105] C.G. Speziale, S. Sarkar, and T.B. Gatski. Modelling the pressure-strain correlation of turbulence: an invariant dynamical systems approach. Journal of Fluid Mechanics, 227:245-272, 1991.

[106] H.B. Squire. Analysis of the vortex breakdown phenomenon. part 1. Technical report, Imperial College, Aeronautical Department, 1960. report No. 102.

[107] W. Steenbergen. Turbulent Pipe Flow with Swirl. PhD thesis, Eindhoven University of Technology, 1996.

[108] G.I. Taylor. The Viscosity of a Fluid Containing Small Drops of Another Fluid. Proceedings of the Royal Society of London. Series A, 138(834):41-48, 1932.

[109] G.I. Taylor. The Formation of Emulsions in Definable Fields of Flow. Proceedings of the Royal Society of London A, 146:501-523, 1934.

[110] R.M. Thomas. Bubble coalescence in turbulent flows. International Journal of Multiphase Flow, 7(6):709 - 717, 1981.

[111] J.F. Thompson, B.K. Soni, and N.P. Weatherill, editors. Handbook of Grid Generation. CRC Press, 1999.

[112] Harald Thunem. Coalescence in Dense Water/Oil Dispersions. PhD thesis, Norges Teknische Høgskole, 1993.

[113] M.B.H. Van Noordenburg. On Leading-Edge Vortex Flow and Vortex Breakdown. PhD thesis, University of Twente, 1999. 
[114] N. Vankova, S. Tcholakova, N.D. Denkov, I.B. Ivanov, V.D. Vulchev, and T. Danner. Emulsification in turbulent flow: 1. Mean and maximum drop diameters in inertial and viscous regimes. Journal of Colloid and Interface Science, 312(2):363 - 380, 2007.

[115] D.C. Wilcox. Turbulence Modeling for CFD. DCW Industries, INC, 2006.

[116] M. Worner. A Compact Introduction to the Numerical Modeling of Multiphase Flows. Technical report, Forschungzentrum Karlsruhe, 2003.

[117] G.A.B. Young, W.D. Wakley, D.L. Taggart, S.L. Andrews, and J.R. Worrell. Oilwater separation using hydrocyclones: An experimental search for optimum dimensions. Journal of Petroleum Science and Engineering, 11(1):37 - 50, 1994.

[118] M.J. van der Zande. Droplet break-up in turbulent oil-in-water flow through a restriction. PhD thesis, Delft University of Technology, 2000. 

The shape of the nose section is expressed as:

$$
\begin{aligned}
r\left(\xi_{n}\right)=R_{i n}[ & b_{1} \xi_{n}^{1 / 2}+b_{2} \xi_{n}^{3 / 2}+f_{n}(0) P_{1}\left(\xi_{n}\right) \\
& \left.+f_{n}^{\prime}(0) P_{3}\left(\xi_{n}\right)+f_{n}(1) P_{2}\left(\xi_{n}\right)+f_{n}^{\prime}(1) P_{4}\left(\xi_{n}\right)\right]
\end{aligned}
$$

Here $\xi_{n}$ is the dimensionless coordinate with $\xi_{n} \in[0,1]$

$$
\xi_{n}=\frac{x_{n}-x_{n o s e}}{x_{\text {vanes }}-x_{\text {nose }}}
$$

Cubic Hermite polynomials $P_{1}$ to $P_{4}$ are given by:

$$
\begin{aligned}
& P_{1}\left(\xi_{n}\right)=\left(1-\xi_{n}\right)^{2}\left(1+2 \xi_{n}\right), \\
& P_{2}\left(\xi_{n}\right)=1-P_{1}\left(\xi_{n}\right) \\
& P_{3}\left(\xi_{n}\right)=\left(1-\xi_{n}\right)^{2} \xi_{n} \\
& P_{4}\left(\xi_{n}\right)=-\xi_{n}^{2}\left(1-\xi_{n}\right)
\end{aligned}
$$

The slope of the central body is:

$$
\begin{aligned}
\frac{d r\left(\xi_{n}\right)}{d x_{n}}=\frac{R_{i n}}{\Delta x_{n}} & {\left[1 / 2 b_{1} \xi_{n}^{-1 / 2}+3 / 2 b_{2} \xi_{n}^{1 / 2}+f_{n}(0) P_{1}^{\prime}\left(\xi_{n}\right)\right.} \\
& \left.+f_{n}^{\prime}(0) P_{3}^{\prime}\left(\xi_{n}\right)+f_{n}(1) P_{2}^{\prime}\left(\xi_{n}\right)+f_{n}^{\prime}(1) P_{4}^{\prime}\left(\xi_{n}\right)\right]
\end{aligned}
$$

Here $\Delta x_{n}=x_{\text {vanes }}-x_{n o s e}=80 \mathrm{~mm}$. The first derivatives of the cubic Hermite polynomials are given by 


$$
\begin{aligned}
& P_{1}^{\prime}\left(\xi_{n}\right)=-6\left(1-\xi_{n}\right) \xi_{n}, \\
& P_{2}^{\prime}\left(\xi_{n}\right)=-P_{1}^{\prime}\left(\xi_{n}\right) \\
& P_{3}^{\prime}\left(\xi_{n}\right)=\left(1-\xi_{n}\right)\left(1-3 \xi_{n}\right), \\
& P_{4}^{\prime}\left(\xi_{n}\right)=\xi_{n}\left(-2+3 \xi_{n}\right)
\end{aligned}
$$

The second derivative follows as:

$$
\begin{aligned}
\frac{d^{2} r\left(\xi_{n}\right)}{d x_{n}^{2}}=\frac{R_{i n}}{\Delta x_{n}^{2}}[ & -1 / 4 b_{1} \xi_{n}^{-3 / 2}+3 / 4 b_{2} \xi_{n}^{-1 / 2}+f_{n}(0) P_{1}^{\prime \prime}\left(\xi_{n}\right) \\
& \left.+f_{n}^{\prime}(0) P_{3}^{\prime \prime}\left(\xi_{n}\right)+f_{n}(1) P_{2}^{\prime \prime}\left(\xi_{n}\right)+f_{n}^{\prime}(1) P_{4}^{\prime \prime}\left(\xi_{n}\right)\right]
\end{aligned}
$$

The second derivatives of the cubic Hermite polynomials are given by

$$
\begin{aligned}
& P_{1}^{\prime \prime}\left(\xi_{n}\right)=-6+12 \xi_{n}, \\
& P_{2}^{\prime \prime}\left(\xi_{n}\right)=-P_{1}^{\prime \prime}\left(\xi_{n}\right) \\
& P_{3}^{\prime \prime}\left(\xi_{n}\right)=-4+6 \xi_{n} \\
& P_{4}^{\prime \prime}\left(\xi_{n}\right)=-2+6 \xi_{n}
\end{aligned}
$$

For $\mathrm{r}\left(\xi_{n}\right)$ the following conditions apply:

$$
\begin{aligned}
\left.r\right|_{\xi_{n}=0} & =0, \\
\left.r\right|_{\xi_{n}=1} & =R_{i n}, \\
\left.\frac{d r}{d x_{n}}\right|_{\xi_{n}=1} & =0 \\
\left.\frac{d^{2} r}{d x_{n}^{2}}\right|_{\xi_{n}=1} & =0
\end{aligned}
$$

This leads to

$$
\begin{array}{r}
f_{n}(0)=0 \\
b_{1}+b_{2}+f_{n}(1)=1 \\
1 / 2 b_{1}+3 / 2 b_{2}+f_{n}^{\prime}(1)=0 \\
-1 / 4 b_{1}+3 / 4 b_{2}+f_{n}(0)(6)+f_{n}^{\prime}(0)(2)+f_{n}(1)(-6)+f_{n}^{\prime}(1)(4)=0
\end{array}
$$

These equations can be solved for the amplitudes of the Hermite polynomials as:

$$
\begin{aligned}
& f_{n}(0)=0 \\
& f_{n}^{\prime}(0)=3-15 / 8 b_{1}-3 / 8 b_{2} \\
& f_{n}(1)=1-b_{1}-b_{2} \\
& f_{n}^{\prime}(1)=-1 / 2 b_{1}-3 / 2 b_{2}
\end{aligned}
$$

Substitution of these results with $b_{1}=0.9$ and $b_{2}=6.0$ into the equation for the nose section leads to the final shape. The constants $b_{1}$ and $b_{2}$ are determined by trial and error in order to obtain a suitable shape. 
APPENDIX B

\section{Design of tail section with quintic Hermite polynomials}

The shape of the tail section is expressed as:

$$
\begin{aligned}
r\left(\xi_{t}\right)=R_{i n}[ & f_{t}(0) Q_{1}\left(\xi_{t}\right)+f_{t}^{\prime}(0) Q_{3}\left(\xi_{t}\right)+f_{t}^{\prime \prime}(0) Q_{5}\left(\xi_{t}\right) \\
& \left.+f_{t}(1) Q_{2}\left(\xi_{t}\right)+f_{t}^{\prime}(1) Q_{4}\left(\xi_{t}\right)+f_{t}^{\prime \prime}(1) Q_{6}\left(\xi_{t}\right)\right]
\end{aligned}
$$

The dimensionless axial coordinate $\xi_{t}$ defined as is

$$
\xi_{t}=\frac{x_{t}-x_{t a i l}}{\Delta x_{t}}=\frac{x_{t}-x_{t a i l}}{x_{t i p}-x_{t a i l}}
$$

Here $\Delta x_{t}=x_{t i p}-x_{t a i l}=200 \mathrm{~mm} . Q_{1}$ to $Q_{6}$ are the quintic Hermite polynomials given by:

$$
\begin{aligned}
& Q_{1}\left(\xi_{t}\right)=\left(1-\xi_{t}\right)^{3}\left(1+3 \xi_{t}+6 \xi_{t}^{2}\right) \\
& Q_{2}\left(\xi_{t}\right)=1-Q_{1}\left(\xi_{t}\right) \\
& Q_{3}\left(\xi_{t}\right)=\left(1-\xi_{t}\right)^{3} \xi_{t}\left(1+3 \xi_{t}\right) \\
& Q_{4}\left(\xi_{t}\right)=\xi_{t}^{3}\left(1-\xi_{t}\right)\left(-4+3 \xi_{t}\right) \\
& Q_{5}\left(\xi_{t}\right)=1 / 2\left(1-\xi_{t}\right)^{3} \xi_{t}^{2} \\
& Q_{6}\left(\xi_{t}\right)=1 / 2 \xi_{t}^{3}\left(1-\xi_{t}\right)^{2}
\end{aligned}
$$

The first derivative of the shape function is

$$
\begin{aligned}
\frac{d r\left(\xi_{t}\right)}{d x_{t}}=\frac{R_{\text {in }}}{\Delta x_{t}}[ & f_{t}(0) Q_{1}^{\prime}\left(\xi_{t}\right)+f_{t}^{\prime}(0) Q_{3}^{\prime}\left(\xi_{t}\right)+f_{t}^{\prime \prime}(0) Q_{5}^{\prime}\left(\xi_{t}\right) \\
& \left.+f_{t}(1) Q_{2}^{\prime}\left(\xi_{t}\right)+f_{t}^{\prime}(1) Q_{4}^{\prime}\left(\xi_{t}\right)+f_{t}^{\prime \prime}(1) Q_{6}^{\prime}\left(\xi_{t}\right)\right]
\end{aligned}
$$


The first derivatives of the quintic Hermite polynomials given by:

$$
\begin{aligned}
& Q_{1}^{\prime}\left(\xi_{t}\right)=-30\left(1-\xi_{t}\right)^{2} \xi_{t}^{2} \\
& Q_{2}^{\prime}\left(\xi_{t}\right)=-Q_{1}^{\prime}\left(\xi_{t}\right) \\
& Q_{3}^{\prime}\left(\xi_{t}\right)=\left(1-\xi_{t}\right)^{2}\left(1+2 \xi_{t}-15 \xi_{t}^{2}\right) \\
& Q_{4}^{\prime}\left(\xi_{t}\right)=\xi_{t}^{2}\left(-12+28 \xi_{t}-15 \xi_{t}^{2}\right) \\
& Q_{5}^{\prime}\left(\xi_{t}\right)=1 / 2\left(1-\xi_{t}\right)^{2} \xi_{t}\left(2-5 \xi_{t}\right) \\
& Q_{6}^{\prime}\left(\xi_{t}\right)=1 / 2 \xi_{t}^{2}\left(1-\xi_{t}\right)\left(3-5 \xi_{t}\right)
\end{aligned}
$$

And the second derivative of the shape function is

$$
\begin{aligned}
\frac{d^{2} r\left(\xi_{t}\right)}{d x_{t}^{2}}=\frac{R_{i n}}{\Delta x_{t}^{2}}[ & f_{t}(0) Q_{1}^{\prime \prime}\left(\xi_{t}\right)+f_{t}^{\prime}(0) Q_{3}^{\prime \prime}\left(\xi_{t}\right)+f_{t}^{\prime \prime}(0) Q_{5}^{\prime \prime}\left(\xi_{t}\right) \\
& \left.+f_{t}(1) Q_{2}^{\prime \prime}\left(\xi_{t}\right)+f_{t}^{\prime}(1) Q_{4}^{\prime \prime}\left(\xi_{t}\right)+f_{t}^{\prime \prime}(1) Q_{6}^{\prime \prime}\left(\xi_{t}\right)\right]
\end{aligned}
$$

The second derivatives of the quintic Hermite polynomials given by:

$$
\begin{aligned}
& Q_{1}^{\prime \prime}\left(\xi_{t}\right)=-60\left(1-\xi_{t}\right) \xi_{t}\left(1-2 \xi_{t}\right) \\
& Q_{2}^{\prime \prime}\left(\xi_{t}\right)=-Q_{1}^{\prime \prime}\left(\xi_{t}\right) \\
& Q_{3}^{\prime \prime}\left(\xi_{t}\right)=12 \xi_{t}\left(1-\xi_{t}\right)\left(-3+5 \xi_{t}\right) \\
& Q_{4}^{\prime \prime}\left(\xi_{t}\right)=12 \xi_{t}\left(1-\xi_{t}\right)\left(-2+5 \xi_{t}\right) \\
& Q_{5}^{\prime \prime}\left(\xi_{t}\right)=\left(1-\xi_{t}\right)\left(1-8 \xi_{t}+10 \xi_{t}^{2}\right) \\
& Q_{6}^{\prime \prime}\left(\xi_{t}\right)=\xi_{t}\left(3-12 \xi_{t}+10 \xi_{t}^{2}\right)
\end{aligned}
$$

The requirements on $r\left(x_{t}\right)$ are:

$$
\begin{array}{rlll}
\left.r\right|_{\xi_{t}=0} & =R_{\text {in }} & \Rightarrow & f_{t}(0)=1, \\
\left.r\right|_{\xi_{t}=1} & =0 & \Rightarrow & f_{t}(1)=0, \\
\left.\frac{d r}{d x_{t}}\right|_{\xi_{t}=0} & =0 & \Rightarrow & f_{t}^{\prime}(0)=0, \\
\left.\frac{d r}{d x_{t}}\right|_{\xi_{t}=1} & =-\tan \phi_{t} & \Rightarrow & f_{t}^{\prime}(1)=-\frac{\Delta x_{t}}{R_{i n}} \tan \phi_{t}, \\
\left.\frac{d^{2} r}{d x_{t}^{2}}\right|_{\xi_{t}=0}=0 & \Rightarrow & f_{t}^{\prime \prime}(0)=0, \\
\left.\frac{d^{2} r}{d x_{t}^{2}}\right|_{\xi_{t}=1} & =0 & \Rightarrow & f_{t}^{\prime \prime}(1)=0
\end{array}
$$

The angle $\phi_{t}=10^{\circ}$. upon substitution in equation B.1 the equation for the shape of the tail section becomes

$$
r\left(\xi_{t}\right)=R_{i n} Q_{1}\left(\xi_{t}\right)-\Delta x_{t} \tan \phi_{t} Q_{4}\left(\xi_{t}\right)
$$




\section{APPENDIX C}

\section{Sensitivity of velocity distribution for swirl angle Sw variation}

In section 5.3.2 differences are seen in both $\mathrm{Re}_{\mathrm{D}}$ and $\mathrm{Sw}$ in the comparison of the results for single and two-phase flow. However, the difference in Sw is small, namely less than $2 \%$. To assess the influence of such a small change in Sw an additional single-phase flow simulation has been carried out using the vane-less geometry, which is shown in figure 4.36. The flow in this simulation has the same $\operatorname{Re}_{\mathrm{D}}$ as the single-phase flow case: $\operatorname{Re}_{\mathrm{D}}$ $=1.81 \times 10^{5}$ and the swirl angle Sw of the two-phase flow case: Sw $=6.61$, see table C.1. Figure C.1 shows the radial distribution of the time-averaged axial and azimuthal velocity distribution on a line through the center of the pipe in the cross-plane $z=0.50 \mathrm{~m}$ for $S_{w}=6.72$ and $S_{w}=6.61$, respectively. As can be seen in the figure, the differences in the velocity distribution are relatively small compared to the differences seen in figures 5.16 and 5.17. So the velocity distribution appears to be relatively insensitive for small changes in Sw. The difference in the velocity distributions between the single and twophase flow results is therefore mainly caused by the difference in $\operatorname{Re}_{\mathrm{D}}$ and the separation of the phases.

\begin{tabular}{|l|c|c|c|c|c|}
\hline & $\alpha_{\text {inlet }}$ & $d / D$ & $\mathrm{FS}$ & $\mathrm{Re}_{\mathrm{D}}$ & $\mathrm{Sw}$ \\
\hline \hline Single-phase flow & - & - & 0.30 & $1.81 \times 10^{5}$ & 6.72 \\
vane-less case & - & - & 0.30 & $1.81 \times 10^{5}$ & 6.61 \\
Two-phase flow & 0.25 & $1.00 \times 10^{-3}$ & 0.30 & $0.86 \times 10^{5}$ & 6.61 \\
\hline
\end{tabular}

Table C.1: Dimensionless variables in comparison of single and two-phase flow. 


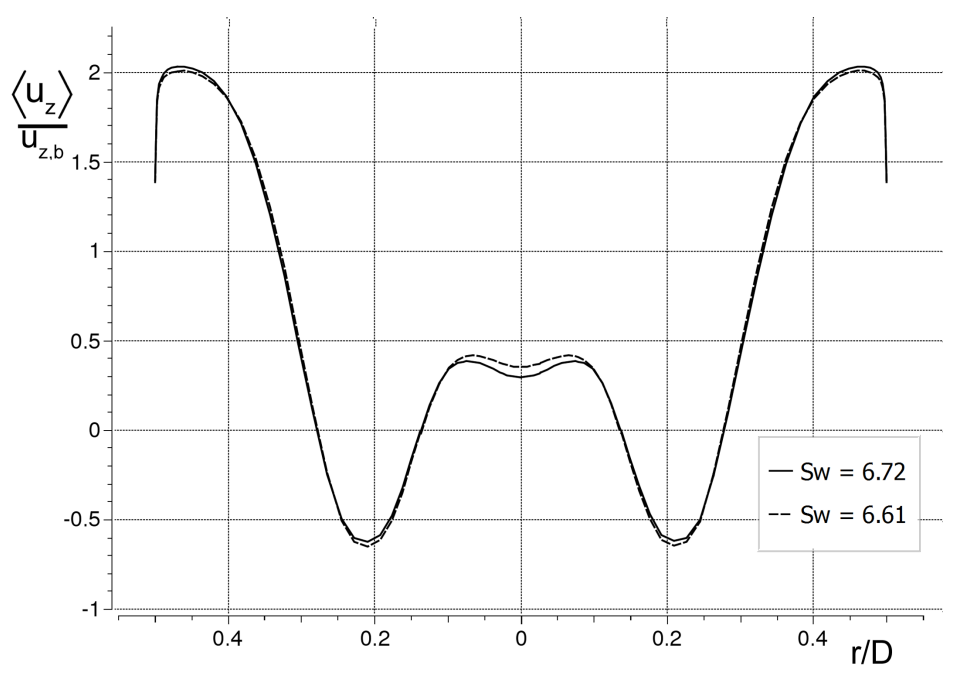

(a) Axial velocity

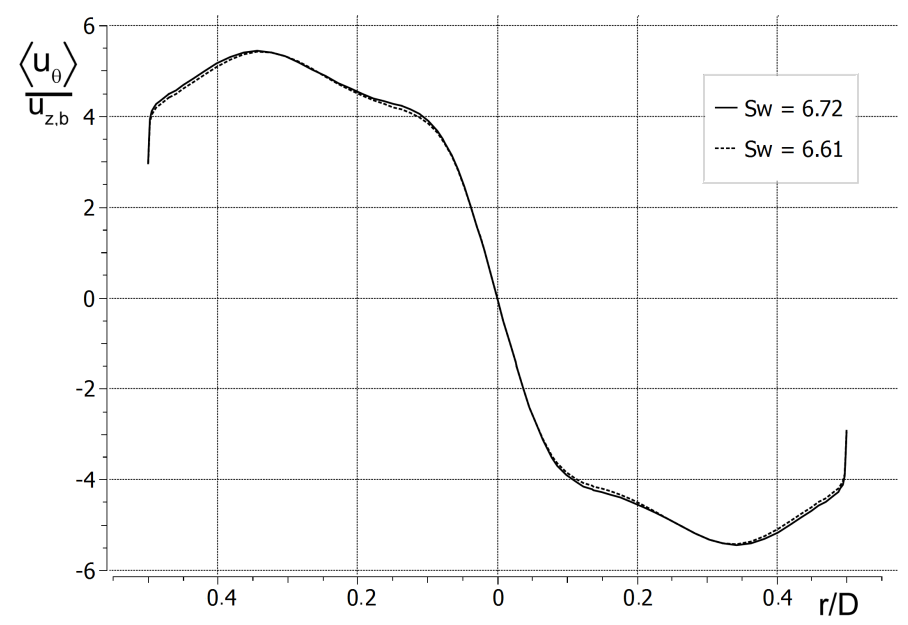

(b) Azimuthal velocity

Figure C.1: Comparison of the radial distribution of the time-averaged axial (top) and azimuthal (bottom) velocity for single-phase water flow on a line through the center of the pipe in the plane $z=0.50 \mathrm{~m}$ for the full geometry case with $\mathrm{Sw}=6.72$ (solid) and the vane-less geometry case with $\mathrm{Sw}=6.61$ (dashed). $\mathrm{FS}=0.30$ and $\mathrm{Re}_{\mathrm{D}}=1.81 \times 10^{5}$. 


\section{Acknowledgments}

The work presented in this thesis was carried out with the support and guidance of many people. Their help was indispensable in completing it. I would like thank everyone who contributed to my research over the past years.

Especially, I would like to thank my supervisor Harry Hoeijmakers. Harry, your advice pointed me in the right direction when I was unsure where to go. Moreover, your relentless eye for detail showed me how to accurately and unambiguously formulate my thoughts on paper. It allowed me to greatly improve this thesis and it will undoubtedly do the same for my future work.

I would also like thanks my fellow researchers Laurens van Campen and Thomas Krebs. The use of your experimental results allowed me to evaluate the outcome of the my numerical simulations, which made my work more valuable. In addition, I would like to thank you for the in-depth discussions we frequently had and the pleasant collaboration in the past years.

I'm grateful for the input I received from the partner companies. Specifically, I would like to thank Wouter Harteveld for introducing the roadmap, which provided direction and more concrete goals in the early stage of the project; Remko Westra for sharing his know-how on liquid-liquid separators and CFD; and Johanna Bos for her feedback during meetings and on my reports, as well as for her role in the day-to-day organizational and administrative aspects of the project. Furthermore, I'm indebted to Paul Verbeek, who managed this complicated project and to Menno Plantenga for his work in initiating the project and for taking care of ISPT-related business. Finally, I would like to express my gratitude to ISPT for creating the opportunity for me to do this work.

Last but not least, I would like to thank the PhD-students, MSc-students and staff members at the Engineering Fluid Dynamics group for the many interactions of both technical and nontechnical nature. You were an audience for my project frustrations, provided me with advice and you were simply good company. 


\section{About the author}

Jesse Slot was born on October 51981 in Enschede. He grew up in Enschede, where he completed his pre-university education at the Ichthus college in 2000.

In the same year he enrolled in Mechanical Engineering at the University of Twente. As his interest for fluid dynamics was triggered by bachelor classes, he choose the master programme 'Engineering Fluid Dynamics'. This research group is led by professor Harry Hoeijmakers.

In 2006 Jesse Slot fulfilled his internship at the Institute of Sound and Vibration Research (ISVR) in Southampton, UK. At the ISVR he worked within the Fluid Dynamics and Acoustics Group on the numerical prediction of noise attenuation in bypass ducts of Rolls-Royce airplane engines under supervision of professor Jeremy Astley and Rie Sugimoto.

For his master graduation project he was involved in the development of computationally efficient multilevel methods for the numerical simulation of sound propagation. His daily supervisor during this project was Kees Venner. Jesse Slot obtained his MSc in Mechanical Engineering cum laude and was award the KIVI-NIRIA price for best dissertation of 2007 for Mechanical Engineering at the University of Twente.

In the summer of 2007 he started his PhD project 'Development of an $\Omega^{2} \mathrm{R}$ separator focussing on oil-water separation' at the University of Twente under supervision of professor Harry Hoeijmakers. The project was organized by the Institute of Sustainable Process Technology (ISPT) and it involved the collaboration between the Universities of Twente, Delft and Wageningen and companies from the oil and gas industry. The results of the project are presented in the present work. During the PhD-project results have been presented by Jesse Slot at the ICMF 2010 in Tampa, Florida and at CFD2011 in Trondheim, Norway.

As of September 2012 Jesse Slot is project manager CFD ships at the Maritime Research Institute Netherlands (Marin) in Wageningen. 



\section{Stellingen}

Behorende bij het proefschrift

DEVELOPMENT OF A CENTRIFUGAL IN-LINE SEPARATOR FOR OIL-WATER FLOWS

J. J. Slot

22 mei 2013

1. In sterk wervelende pijpstromingen treedt een $\mathrm{W}$-vormige radiale verdeling van de axiale snelheid op met een annulair terugstroomgebied. (Hoofdstuk 4 van dit proefschrift.)

2. Een verandering in de verhouding tussen de azimuthale en axiale snelheid bij het genereren van de wervelstroming in de pijp kan bij een verhouding die laag genoeg is leiden tot een $\mathrm{V}$-vormige radiale verdeling van de axiale snelheid. (Hoofdstuk 4 van dit proefschrift.)

3. Het gebruik van een turbulent dispersie model, dat uitgaat van isotrope turbulentie, leidt in de centrifugaal scheider tot zeer sterke, niet-fysische radiale diffusie van de disperse fase. (Hoofdstuk 5 van dit proefschrift.)

4. Vanwege de gevoeligheid voor de druppelgrootte van de numerieke resultaten voor gedispergeerde twee-fase stromingen, is een goede afschatting van de inlaat druppelgrootte van groot belang voor de voorspelling van de olie-water scheidingsefficiëntie. (Hoofdstuk 5 van dit proefschrift.)

5. Voor het opstellen van een versimpeld en inzichtelijk model van de werkelijkheid is het vaak nodig om veel meer fysica en details mee te nemen in de analyse van het probleem dan het model doet vermoeden.

6. Vele modellen die worden gebruikt in numerieke simulaties van meerfase stromingen zijn ontwikkeld voor gesimplificeerde condities, condities welke helaas zelden voorkomen in industriële toepassingen.

7. In industrie-gestuurde promotie-onderzoeken kunnen het enthousiasme vanuit de industrie en de wens tot participatie van de universiteit leiden tot doelstellingen die onhaalbaar zijn in vier jaar onderzoek. 
8. Als de gebruiker van commerciële computerprogramma's voor numerieke stromingsberekeningen geen kennis heeft van de achterliggende modellen, kan dat voor de gebruiker verstrekkende gevolgen hebben.

9. Het gedetailleerd en projectmatig plannen van een promotie-onderzoek houdt er geen rekening mee dat zowel de uitkomst als het traject daar naar toe in grote mate onontgonnen terrein zijn.

10. De scheiding van olie en water in scheidingsvaten, gemodelleerd als een één-dimensionaal tijdsafhankelijk probleem, kan worden beschreven door middel van de methode van karakteristieken. 